\title{
The spiral structure of our Milky Way Galaxy ${ }^{\star}$
}

\author{
L. G. Hou ${ }^{1,2}$, J. L. Han ${ }^{1}$, and W. B. Shi ${ }^{1,3}$ \\ 1 National Astronomical Observatories, Chinese Academy of Sciences, Jia-20, DaTun Road, Chaoyang District, Beijing 100012, \\ PR China \\ e-mail: hjl@bao.ac.cn \\ 2 Department of Physics, School of Physics, Peking University, Beijing 100871, PR China \\ 3 Department of Space Science and Applied Physics, Shandong University at Weihai, 180 Cultural West Road, Shandong 264209, \\ PR China
}

Received 2 March 2008 / Accepted 20 February 2009

\section{ABSTRACT}

\begin{abstract}
Context. The spiral structure of our Milky Way Galaxy is not yet known. HII regions and giant molecular clouds are the most prominent spiral tracers. Models with 2-4 arms have been proposed to outline the structure of our Galaxy.

Aims. Recently, new data of spiral tracers covering a larger region of the Galactic disk have been published. We wish to outline the spiral structure of the Milky way using all tracer data.

Methods. We collected the spiral tracer data of our Milky Way from the literature, namely, HII regions and giant molecular clouds (GMCs). With weighting factors based on the excitation parameters of HII regions or the masses of GMCs, we fitted the distribution of these tracers with models of two, three, four spiral-arms or polynomial spiral arms. The distances of tracers, if not available from stellar or direct measurements, were estimated kinetically from the standard rotation curve of Brand \& Blitz (1993, A\&A, 275, 67) with $R_{0}=8.5 \mathrm{kpc}$, and $\Theta_{0}=220 \mathrm{~km} \mathrm{~s}^{-1}$ or the newly fitted rotation curves with $R_{0}=8.0 \mathrm{kpc}$ and $\Theta_{0}=220 \mathrm{~km} \mathrm{~s}^{-1}$ or $R_{0}=8.4 \mathrm{kpc}$ and $\Theta_{0}=254 \mathrm{~km} \mathrm{~s}^{-1}$.

Results. We found that the two-arm logarithmic model cannot fit the data in many regions. The three- and the four-arm logarithmic models are able to connect most tracers. However, at least two observed tangential directions cannot be matched by the three- or four-arm model. We composed a polynomial spiral arm model, which can not only fit the tracer distribution but also match observed tangential directions. Using new rotation curves with $R_{0}=8.0 \mathrm{kpc}$ and $\Theta_{0}=220 \mathrm{~km} \mathrm{~s}^{-1}$ and $R_{0}=8.4 \mathrm{kpc}$ and $\Theta_{0}=254 \mathrm{~km} \mathrm{~s}^{-1}$ for the estimation of kinematic distances, we found that the distribution of HII regions and GMCs can fit the models well, although the results do not change significantly compared to the parameters with the standard $R_{0}$ and $\Theta_{0}$.
\end{abstract}

Key words. Galaxy: structure - Galaxy: kinematics and dynamics - ISM: HII regions

\section{Introduction}

The Milky Way galaxy is known to be a spiral galaxy, but its detailed spiral structure has not been well revealed. The Milky Way may have two arms, or three arms, or even more complicated structures (Vallée 2008). At present, the number of arms and arm parameters (i.e. the pitch angle and the initial Galactocentric radius) have not been well determined. Nevertheless, some consensus on the Galactic structure has been reached (Russeil 2003, hereafter R03): (1) the tangential directions of the spiral arms have been identified from the maxima of the thermal radio continuum and molecular emission (see Table 1 in Englmaier \& Gerhard 1999; or Table 2 in Vallée 2008); (2) the Sagittarius arm and the Carina arm are linked as a single arm; (3) the Sun lies between the Perseus arm and Sagittarius arm.

To outline the structure of our Galaxy, one should use all reliable tracers with accurate distances. Primary tracers are:

(1) HII regions. HII regions are the birthplace of young stars. They are clouds of atomic hydrogen ionized by bright young stars. Their radio emission cannot be attenuated by dust extinction, and therefore they can be detected even in distant parts of the Galactic plane. Georgelin \& Georgelin (1976) investigated the distribution of the available sample of HII regions that

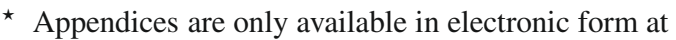
http://www . aanda.org
}

time, and outlined four segments of arms. Downes et al. (1980) observed more HII regions in the first quadrant and Caswell \& Haynes (1987) reported comprehensive data of southern HII regions in the Galaxy over the Galactic longitude $l=210^{\circ}$ to $l=360^{\circ}$, which help to delineate the spiral structure, especially for the Carina arm and the Crux arm.

(2) Giant molecular clouds (GMCs). The GMCs are vast assemblies of molecular gas with a mass of $10^{4} \sim 10^{6} M_{\odot}$ and a size of tens of pc. They have an average density of $10^{2} \sim 10^{3} \mathrm{~cm}^{-3}$ but their core density can reach $10^{6} \sim 10^{7} \mathrm{~cm}^{-3}$. Because the interstellar medium is almost optically thin for the CO emission line, distant clouds in the Galactic plane can be detected. Cohen et al. (1986) showed that the GMCs are good tracers of the Carina arm. Dame et al. (1986) solved the distance ambiguity of some GMCs in the first quadrant, and used them to outline the Sagittarius arm. Solomon et al. (1987) studied warm molecular clouds in the first quadrant, and delineated three armlike structures, the Perseus arm, the Sagittarius arm and another possible one, the Scutum arm. Grabelsky et al. (1988) cataloged GMCs over the Galactic longitude from $l=270^{\circ}$ to $l=300^{\circ}$, and outlined the Sagittarius-Carina arm using GMCs over $23 \mathrm{kpc}$ in the Galactic plane. More GMCs should be identified from the complete CO map of our Galaxy composed by Dame et al. (2001), but unfortunately, this has not yet been done. 
In addition, HI gas is one of the major components of the interstellar medium in spiral galaxies. Levine et al. (2006) decomposed the HI data into the smoothed component and perturbed surface density map, and found a spiral structure out to at least $25 \mathrm{kpc}$. Four arm-segments appear in the outer Milky Way disk.

As shown in external Galaxies, the distribution of the brightest HII regions and of the most massive molecular clouds generally traces the grand design structure. To our knowledge, previous authors examining the spiral structure of the Milky Way used only HII regions or GMCs, and none considered all tracers together for spiral structure, except for R03 who considered the complexes of HII regions and molecular clouds. R03 showed the distribution of a large sample of star-forming complexes (constituted by HII regions, ionized patches and/or molecular clouds or the mixture of any two), and fitted it with two, three, or four logarithmic spiral arm models. The four-arm model is slightly more preferable than the three-arm model. We collect a cata$\log$ of HII regions and GMCs, and use those tracers together to show the grand design of our Galaxy. In Sect. 2, we discuss spiral tracers and determine their parameters (distance, and the assigned weight - here we mean the weighting factor rather than the mass). In Sect. 3, we present the distribution of the tracers and discuss the models. Conclusions are presented in Sect. 4.

\section{Tracer data for the Galactic spiral structure}

To reveal the structure of our Galaxy, tracers spread over the whole Galactic disk are needed. Also, their distances have to be determined.

\subsection{Tracers for the Galactic spiral structure: data}

For any tracer in the outer Galaxy, if its stellar distance cannot be determined, one can use the kinematic method to estimate the distance. However, for a tracer in the inner Galaxy, the distance ambiguity is a problem. Two possible distances correspond to the same observed radial velocity. Because the distance ambiguity of HII regions can be resolved by $\mathrm{HI} / \mathrm{H}_{2} \mathrm{CO}$ emission/absorption observations or the HI self-absorption method (e.g. Anderson \& Bania 2009; Tian \& Leahy 2008), HII regions are excellent tracers in the inner Galaxy.

In addition to Georgelin \& Georgelin (1976), Downes et al. (1980) and Caswell \& Haynes (1987) on the spiral structure of the Milky Way using HII regions as spiral tracers, Paladini et al. (2003) collected a catalog of Galactic HII regions, which contained 1442 sources. Paladini et al. (2004) presented a new, detailed analysis of the spatial distribution of some Galactic HII regions. Araya et al. (2002), Watson et al. (2003), and Sewilo et al. (2004) reported simultaneous $\mathrm{H} 110 \alpha$ and $\mathrm{H}_{2} \mathrm{CO}$ line observations toward HII regions in the first quadrant and resolved the distance ambiguity. Kuchar \& Bania (1994), Kolpak et al. (2003), Fish et al. (2003), Pandian et al. (2008), Anderson \& Bania (2009) used HI absorption to resolve the distance ambiguity for other samples of HII regions. R03 established a starforming complex catalog, and derived Galactic structure. Russeil et al. (2007) revised distances of 32 HII regions.

For GMCs, besides Dame et al. (1986), Solomon et al. (1987) and Grabelsky et al. (1988) for spiral structure of the Milky Way, there are also investigations on Galactic molecular clouds. Mead \& Kutner (1988) reported 31 clouds in the first quadrant outside the solar circle. Digel et al. (1990) observed 32 clouds, related to the Outer arm in the first quadrant. Sodroski (1991) studied 35 clouds located in the second, third and fourth quadrants. May et al. (1997) identified 177 clouds in the third quadrant, and shown disk structure as well as warping of the disk. Carpenter et al. (1990) studied 18 molecular clouds in the outer Galactic disk. Brand \& Wouterloot (1994) detected a sample of molecular clouds located in the far outer Milky Way. Nakagawa et al. (2005) revealed 70 molecular clouds in the Galactic Warp with a kinematic distance greater than about $14.5 \mathrm{kpc}$. Heyer et al. (2001) summarized the properties of molecular regions in the outer galaxy from the Five College Radio Astronomy Observatory Outer Galaxy Survey.

We have collected the published data of HII regions and GMCs from the references above, including position, velocity, flux, etc. The stellar distances of HII regions are used when possible, otherwise the kinematic distances are estimated by using three rotation curves (see below), one with the constants $R_{0}=8.5 \mathrm{kpc}$ and $\Theta_{0}=220 \mathrm{~km} \mathrm{~s}^{-1}$, another with $R_{0}=8.0 \mathrm{kpc}$ and $\Theta_{0}=220 \mathrm{~km} \mathrm{~s}^{-1}$ and also the new one with $R_{0}=8.4 \mathrm{kpc}$ and $\Theta_{0}=254 \mathrm{~km} \mathrm{~s}^{-1}$. Here $R_{0}$ is the distance between the Sun and the Galactic Center, and $\Theta_{0}$ is the velocity of the Sun circling around the Galactic Center. The excitation parameters of HII regions and the masses of GMCs are estimated and rescaled to the adopted distances. Clouds with a mass less than $10^{4} M_{\odot}$ are not massive and will not be considered. We have cross-identified the tracers according to their longitudes, latitudes and velocities to avoid redundancy. We list all related parameters of the tracers with references in Tables A.1 and A.2 (online version only).

\subsection{Rotation curves and distances of tracers}

The distance of a given spiral tracer could be best determined by triangulation observations (e.g. Xu et al. 2006, 2008; Brunthaler et al. 2008; Zhang et al. 2008; Reid et al. 2008; Moscadelli et al. 2008). Only a few clouds or HII regions have been so well measured. For many tracers, the distance of the associated bright stars is adopted from the literature. Here, we will use these measurements to verify the rotation curves.

The Galactic rotation curve has been derived from the tracers with their stellar distances and velocities already determined. In the last 20 years, four rotation curves have been used for the spiral structure of the Milky Way: one for the whole Galaxy from Brand \& Blitz (1993, hereafter BB93), the two for the north part of the Galaxy from Clemens (1985) and Fich et al. (1989), and the very simple flat rotation curve with $\Theta=220 \mathrm{~km} \mathrm{~s}^{-1}$ for the whole Galaxy. R03 obtained a Galactic rotation curve by using his sample of star-forming complexes, which is almost the same as that of BB93.

Using the sources with stellar distances in our collected sample, we verified the four possible rotation curves mentioned above with the IAU standard values, $R_{0}=8.5 \mathrm{kpc}$ and $\Theta_{0}=$ $220 \mathrm{~km} \mathrm{~s}^{-1}$. Similarly to R03, we calculated the parameter $\tau$ :

$\tau=\frac{1}{N} \sum_{i=1}^{N} \sqrt{\frac{\left(R_{i}-R_{t}\right)^{2}}{\sigma_{R_{i}}^{2}}+\frac{\left(\omega_{i}-\omega_{t}\right)^{2}}{\sigma_{\omega_{i}}^{2}}}$.

Here, $R_{i}$ and $\omega_{i}$ are the Galactocentric distance and the angular rotation velocity of a tracer, respectively, and $\sigma_{R_{i}}$ and $\sigma_{\omega_{i}}$ are their uncertainties. $R_{\mathrm{t}}, \omega_{\mathrm{t}}$ are the coordinates of the theoretical points. The most appropriate rotation curve should minimize the parameter $\tau$. We found that the rotation curve of Clemens (1985) can indeed minimize the parameter $\tau$ for the north part and the whole Galaxy with a polynomial fit. However it fits all the structures of the curve, even those caused by local motions. The $\tau$ for the rotation curve of Fich et al. (1989) and the simple flat one are larger than that of BB93 both in the north part and 


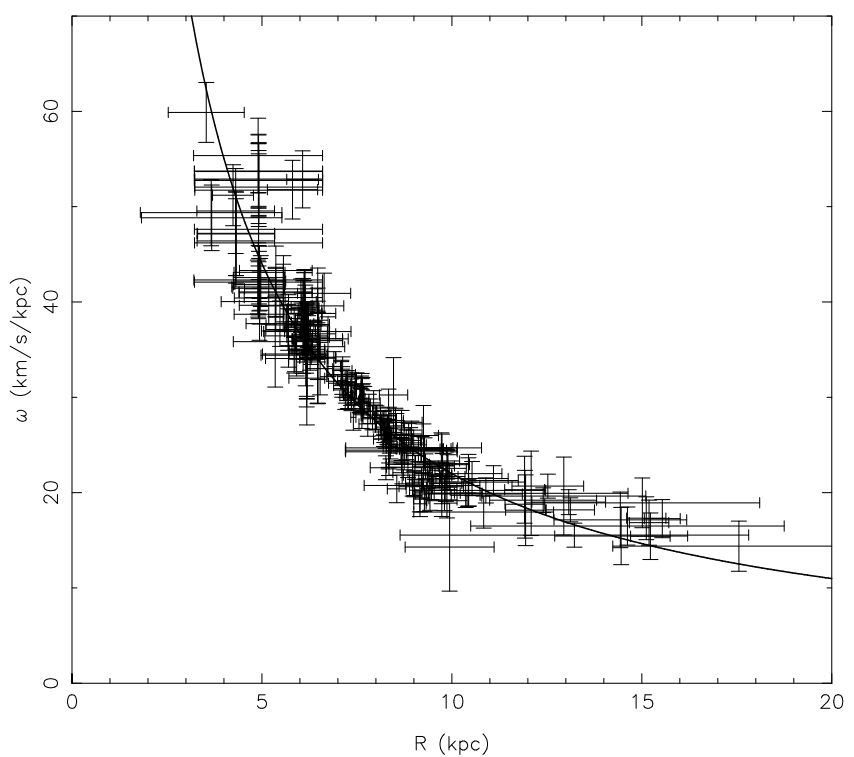

Fig. 1. The rotation curve fitted with solar parameters $R_{0}=8.0 \mathrm{kpc}$ and $\Theta_{0}=220 \mathrm{~km} \mathrm{~s}^{-1}$. Data are taken from our collected sample listed in Tables A.1 and A.2.

whole Galaxy. The rotation curve of BB93 (i.e. Eq. (2) below with $a=1.00767, b=0.0394$ and $c=0.00712$ ) is reasonable for all data of the whole Galaxy. We will directly adopt it to estimate the kinematic distances of the spiral tracers for the case with $R_{0}=8.5 \mathrm{kpc}$ and $\Theta_{0}=220 \mathrm{~km} \mathrm{~s}^{-1}$.

More and more pieces of evidence of $R_{0}=8.0 \mathrm{kpc}$ and $\Theta_{0}=220 \mathrm{~km} \mathrm{~s}^{-1}$ have been found (Reid 1993; Ghez et al. 2008; Eisenhauer et al. 2003; Groenewegen et al. 2008; Gillessen et al. 2009). The change of these constants may affect the kinematic distances of tracers and then affect the derived structure of the Milky Way. Therefore, we fit a new rotation curve with (see Brand \& Blitz 1993, R03):

$\omega / \omega_{0}=a\left(R / R_{0}\right)^{b-1}+c\left(R_{0} / R\right)$.

We set the rotation curve to pass through the new $R_{0}$ and $\Theta_{0}$, and set $c=1-a$. By minimizing $\tau$ in Eq. (1), we found the best values, $a=1.59073, b=-0.000816$. The result is shown in Fig. 1. In Sect. 3.3, we will use this rotation curve, and also the newly determined one with $R_{0}=8.4 \mathrm{kpc}$ and $\Theta_{0}=254 \mathrm{~km} \mathrm{~s}^{-1}$ by Reid et al. (2009), to estimate the kinematic distances and related parameters, and outline the spiral structure. Note that Gillessen et al. (2009) obtained $R_{0}=8.33 \pm 0.35 \mathrm{kpc}$ from the combined fitting to observations of 16 years for stellar orbits around the black hole in the Galactic center.

\subsection{Tracers of Galactic spiral structure: weights}

The brighter the HII region, the better it is as a tracer of spiral structure. We use the excitation parameters of HII regions as a weighting factor to demonstrate spiral structure. Georgelin \& Georgelin (1976) delineated four arm-segments using HII regions with an excitation parameter $U$ greater than $70 \mathrm{pc} \mathrm{cm}^{-2}$. R03 used the excitation parameter as a weighting factor in their fitting process. The excitation parameter $U$ (in $\mathrm{pc} \mathrm{cm}^{-2}$ ) is defined as (Schraml \& Mezger 1969):

$U=4.5526 \alpha(v, T)^{-1} v^{0.1} T^{0.35} S_{v} D^{2}$.

Here, $T$ is the temperature (in $\mathrm{K}$ ), $S_{v}$ is the radio flux density (in Jy), $v$ is the frequency (in $\mathrm{GHz}$ ), $D$ is the distance of the
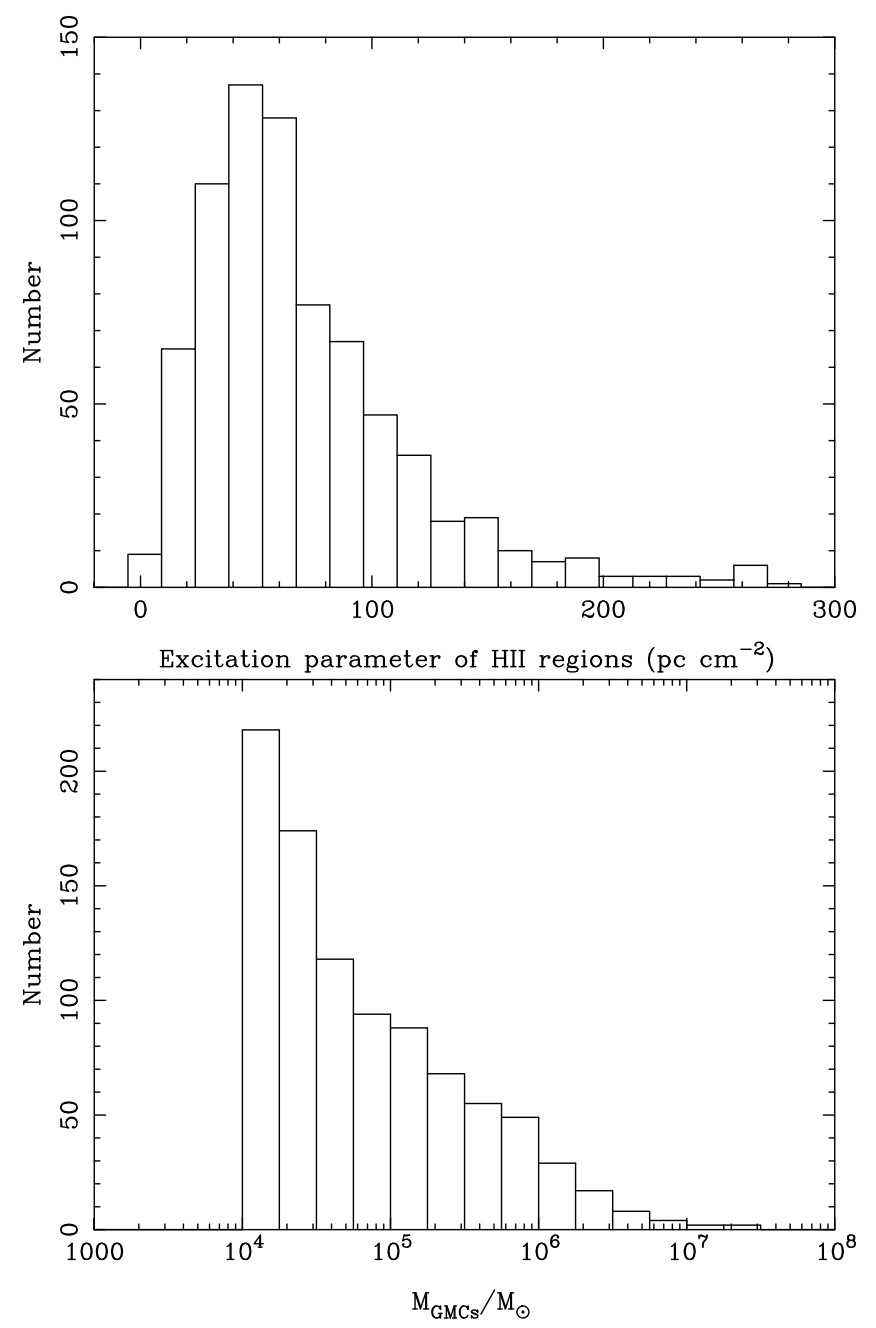

Fig. 2. The distribution of excitation parameters of the HII regions (top panel) and masses of molecular clouds (bottom panel). The rotation curve with $R_{0}=8.5 \mathrm{kpc}$ and $\Theta_{0}=220 \mathrm{~km} \mathrm{~s}^{-1}$ was used if the distances of these tracers were estimated kinetically.

tracers to the Sun (in $\mathrm{kpc}$ ), and $\alpha(v, T)$ is a parameter close to 1 (Schraml \& Mezger 1969). We collected the $S_{v}$ from the literature when available. The excitation parameter $U$ is then estimated via Eq. (3) using $S_{v}$ and assuming $T=8500 \mathrm{~K}$. For a temperature in the range of $7000 \sim 10000 \mathrm{~K}$ the value of excitation parameter differs by less than about $6 \%$. The distribution of excitation parameters of HII regions in our sample is shown in Fig. 2. It ranges from 5 to $332 \mathrm{pc} \mathrm{cm}^{-2}$ with a peak around $50 \mathrm{pc} \mathrm{cm}^{-2}$.

Massive molecular clouds are more concentrated to in the spiral arms than clouds with low masses. The mass of a molecular cloud $M_{\mathrm{GMCs}}$ was rescaled with newly adopted distances. The distribution of $M_{\mathrm{GMCs}}$ is shown in Fig. 2, ranging from $1 \times$ $10^{4} M_{\odot}$ to $2.5 \times 10^{7} M_{\odot}$. More clouds have a lower mass.

In order to use the HII regions and GMCs together to outline the spiral structure of our Galaxy, a reasonable match of weighting parameters should be considered to measure their relative importance to the spiral structure. Obviously, a HII region with a higher excitation parameter or a GMC with a larger $M_{\mathrm{GMCs}}$ should have a larger value of weight. For the HII regions, we take the weighting parameter $B=U /\left(100 \mathrm{pc} \mathrm{cm}^{-2}\right)$, so that an HII region with $U=100 \mathrm{pc} \mathrm{cm}^{-2}$ has a weight of 1 in the determination of the spiral pattern, while an HII region with $U=200 \mathrm{pc} \mathrm{cm}^{-2}$ has a weight of 2 . For the GMCs, the weighting parameter is 

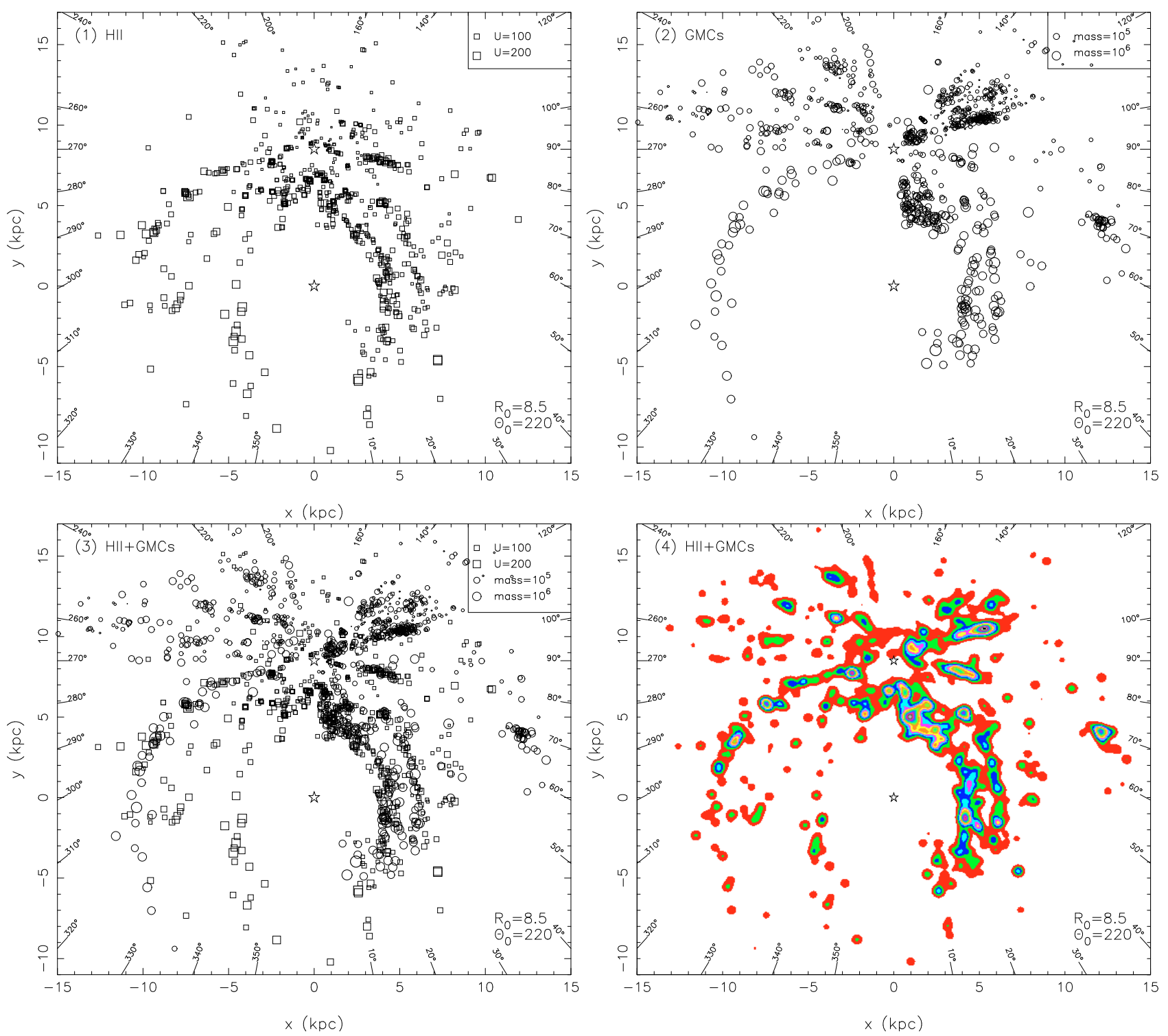

Fig. 3. Panel (1) is the distribution of HII regions, Panel (2) is the distribution of GMCs, and Panel (3) is the distribution of HII regions and GMCs together for illustration of the Galactic spiral structure. The solar parameters $R_{0}=8.5 \mathrm{kpc}$ and $\Theta_{0}=220 \mathrm{~km} \mathrm{~s}^{-1}$ were adopted. The coordinates originate from the Galactic center, and the Sun is located at $(x=0.0 \mathrm{kpc}, y=8.5 \mathrm{kpc})$. The open squares indicate the HII regions with the symbol area proportional to exciting parameters. The open circles indicate GMCs with the symbol size proportional to $\log \left(M_{\mathrm{GMCs}}\right)(\mathrm{see} \operatorname{Sect.} 2.3)$. Panel (4) is the color distribution of both kinds of tracers, each brightened as a Gaussian with the amplitude of the weighting parameter, $B$, so that the spiral arms are clearly demonstrated.

taken to be $B=\log \left(M_{\mathrm{GMCs}} / 10^{4} M_{\odot}\right)$, so that the clouds with a mass of $10^{5} M_{\odot}$ have a weight of $B=1$, and clouds of $10^{6} M_{\odot}$ have a weight of 2 . We have also tried other weighting measures, such as $B=U /\left(50 \mathrm{pc} \mathrm{cm}^{-2}\right)$ and $B=2 \log \left(M_{\mathrm{GMCs}} / 10^{4} M_{\odot}\right)$, and found that the arm structure is similar but with more emphasis on GMCs.

An additional factor needed in the spiral fitting is the distance uncertainty of each tracer. If the uncertainty of observed stellar distance is known, we use it directly. If the kinematic distance is used, we adopt a systemic velocity uncertainty of $\pm 5 \mathrm{~km} \mathrm{~s}^{-1}$, and then the distance uncertainty can be derived by using a rotation curve. As we search for the best match of spiral arms in $X-Y$ coordinates, distance uncertainty will be expressed in $X$ and $Y$. We set a fitting weighting factor $w_{x}=0.5 / \sigma_{x}$ and $w_{y}=0.5 / \sigma_{y}$. We assign all tracers with a distance accuracy better than $0.5 \mathrm{kpc}$ (i.e. $\sigma_{x}<0.5 \mathrm{kpc}$ ) to have $w_{x}=1$, and $w_{y}=1$ if $\sigma_{y}<0.5 \mathrm{kpc}$.

\section{The spiral arms of the Milky Way}

Figure 3 shows the distribution of tracers for the Galactic spiral structure projected onto the Galactic plane. The sizes of symbols have been scaled according to the weighting factors $B$ obtained from excitation parameter $U$ or cloud mass. GMCs in a large region of the fourth Galactic quadrant have been mapped by Dame et al. (2001), but unfortunately, the discrete GMCs have not been identified and explicitly listed in the literature. To show spiral arms better using available data of HII regions and GMCs, in the fourth panel of Fig. 3, we use a Gaussian function to brighten each tracer through:

$L(x, y)=\sum_{i} \frac{B(i)}{\sqrt{2 \pi \sigma^{2}}} \exp \left(-\frac{\left(x_{i}-x\right)^{2}+\left(y_{i}-y\right)^{2}}{2 \sigma^{2}}\right)$.

Here we took $\sigma=0.2$, which means that each tracer will brighten an area with a radius of about $200 \mathrm{pc}$. This is larger 
Table 1. The parameters of the best fitting models: the initial radius, $R_{i}$, the start azimuthal angle, $\theta_{i}$, and the pitch angle, $\psi_{i}$, for the $i$ th arm.

\begin{tabular}{cccccccccccccc}
\hline \hline Model & $\begin{array}{c}R_{1} \\
\mathrm{kpc})\end{array}$ & $\begin{array}{c}\theta_{1} \\
\left({ }^{\circ}\right)\end{array}$ & $\begin{array}{c}\psi_{1} \\
\left({ }^{\circ}\right)\end{array}$ & $\begin{array}{c}R_{2} \\
(\mathrm{kpc})\end{array}$ & $\begin{array}{c}\theta_{2} \\
\left({ }^{\circ}\right)\end{array}$ & $\begin{array}{c}\Psi_{2} \\
\left({ }^{\circ}\right)\end{array}$ & $\begin{array}{c}R_{3} \\
(\mathrm{kpc})\end{array}$ & $\begin{array}{c}\theta_{3} \\
\left({ }^{\circ}\right)\end{array}$ & $\begin{array}{c}\psi_{3} \\
\left({ }^{\circ}\right)\end{array}$ & $\begin{array}{c}R_{4} \\
\left({ }^{\circ}\right)\end{array}$ & $\begin{array}{c}\theta_{4} \\
\left({ }^{\circ}\right)\end{array}$ & $\begin{array}{c}\psi_{4} \\
(\mathrm{kpc})\end{array}$ & $Z$ \\
\hline \\
For distribution of tracers with $R_{0}=8.5 \mathrm{kpc}$ and $\Theta_{0}=220 \mathrm{~km} \mathrm{~s}^{-1}$ \\
2-arm model & 4.2 & 147 & 5.3 & 4.0 & 297 & 5.2 & & & & & & \\
3-arm model & 3.8 & 40 & 7.8 & 3.6 & 195 & 9.9 & 4.0 & 303 & 7.5 & & & & 0.47 \\
4-arm model & 3.7 & 40 & 9.6 & 4.5 & 205 & 10.7 & 4.4 & 290 & 11.4 & 3.9 & 309 & 8.7 & 0.45 \\
\hline
\end{tabular}

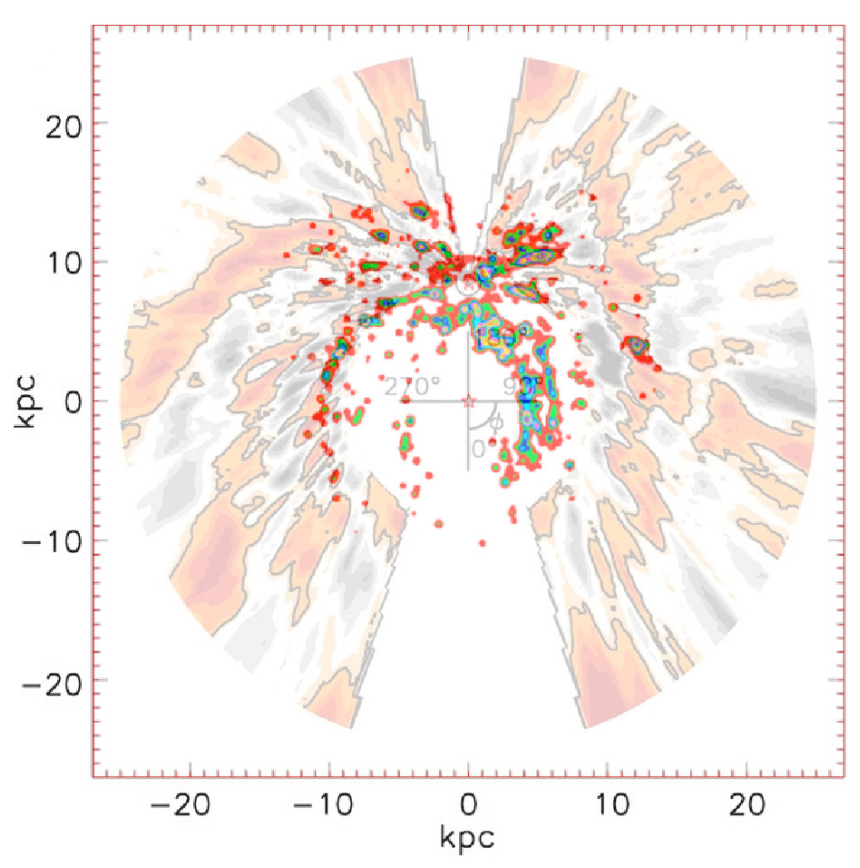

Fig. 4. The spiral structure of HII regions and GMCs $\left(R_{0}=8.5 \mathrm{kpc}\right.$ and $\Theta_{0}=220 \mathrm{~km} \mathrm{~s}^{-1}$ ) is overlaid on the HI map (Levine et al. 2006).

than the size of a GMC, but we can delineate the spiral structure more clearly. A different value of $\sigma$ in a suitable range (e.g. 50-400 pc) produces a similar figure. In the fourth Galactic quadrant, three arm-like structures exist in this region. The Carina arm $\left(l \sim 290^{\circ}\right)$ is very clear. The other two arm-like structures correspond to the Crux $\left(l \sim 318^{\circ}\right)$ and the Norma $\operatorname{arm}\left(l \sim 333^{\circ}\right)$, respectively. In the first quadrant, both HII regions and GMCs also seem to show three arm-like structures, the Sagittarius arm in the middle $\left(l \sim 40^{\circ}\right)$, the Scutum arm inside $\left(l \sim 25^{\circ}\right)$ as well as the Perseus arm on the outer side $\left(x \sim 8 \mathrm{kpc}, l \sim 60^{\circ}\right)$. These arms are clear in Watson et al. (2003) and Sewilo et al. (2004) from HII region distributions and in Solomon et al. (1987) from the distribution of molecular clouds. There may be another arm-like segment in the far outer Galaxy traced by GMCs. In the second and third Galactic quadrants, no obvious structure can be identified from the distribution of the main tracers.

In Fig. 4, we overlay our data onto the HI map of Levine et al. (2006), which was obtained with the solar parameters of $R_{0}=8.5 \mathrm{kpc}, \Theta_{0}=220 \mathrm{~km} \mathrm{~s}^{-1}$. In the HI distribution, there are 3 obvious arm-like structures in the third and fourth quadrants. The Carina arm overlaps with the most inner arm-like structure in the HI distribution, and the Perseus arm may be linked to the intermediate arm-like structure, which can be matched in our 3-arm, 4-arm, and polynomial logarithmic arm models. The counterpart for the outer arm-like structure in the HI map is difficult to identify, but most likely there are one or two spiral arms beyond the Perseus arm.

It is not clear whether the spiral structure of the Milky Way is of logarithmic nature. Canonically, the two, three or four logarithmic arms have been used to fit the tracer data and to model the grand design of our Galaxy. We will try to fit these conventional models to the data, and we also present the polynomial logarithmic arm model with varying pitch angle. In addition to fitting the model to the tracer distribution, it is important to compare the tangential directions of modelled spiral arms to the observed tangents (see Englmaier \& Gerhard 1999; Bronfman 1992; Vallée 2008).

In Sect. 3.3, we also show the distribution of tracers for spiral arms by taking the kinematic distances estimated by using the rotation curves with two sets of solar parameters, $R_{0}=8.0 \mathrm{kpc}$ and $\Theta_{0}=220 \mathrm{~km} \mathrm{~s}^{-1}$ and the recent determination of $R_{0}=8.4 \mathrm{kpc}$ and $\Theta_{0}=254 \mathrm{~km} \mathrm{~s}^{-1}$ from Reid et al. (2009).

\subsection{The logarithmic arm models}

The logarithmic-arm model describes the $i$ th spiral arm with:

$\ln \frac{r}{R_{i}}=\left(\theta-\theta_{i}\right) \tan \psi_{i}$

Here, $r, \theta$ are the polar coordinates centered at the Galactic center, $R_{i}$ is the initial radius (in $\mathrm{kpc}$ ) at the start azimuthal angle $\theta_{i}$ for the $i$ th arm, and $\psi_{i}$ is the pitch angle of the arm. The $\theta$ starts at the positive $x$-axis and increases counterclockwise. We consider the expression:

$Z=\frac{1}{\sum B_{i}} \sum B_{i} \sqrt{\left(x_{i}-x_{t}\right)^{2} w_{x_{i}}^{2}+\left(y_{i}-y_{t}\right)^{2} w_{y_{i}}^{2}}$

for all tracers; here $x_{i}$ and $y_{i}$ are Cartesian coordinates of the tracer, $x_{t}$ and $y_{t}$ are the coordinates of the nearest point from all spiral arms to the tracer. $w_{x_{i}}$ and $w_{y_{i}}$ are weighting factors for the location uncertainty of $x_{i}$ and $y_{i}$, respectively, and $B_{i}$ is the weighting factor related to the excitation parameters of HII regions or the masses of GMCs. The best spiral arm model should be able to minimize the parameter $Z$.

We assume that each arm always has its own pitch angle in the 2-, 3-, 4-arm models and fit them to the data (see also $\mathrm{NaOz}$ $\&$ Shaviv 2007). By searching for the best parameters of each arm, $R_{i}, \theta_{i}$ and $\psi_{i}$ in reasonable ranges, to find the minimized $Z$, we obtained the best spiral arm models as listed in Table 1. Three best-fitting models together with the data are plotted in Fig. 5. The tangential directions of arms in all models are listed in Table 2. 

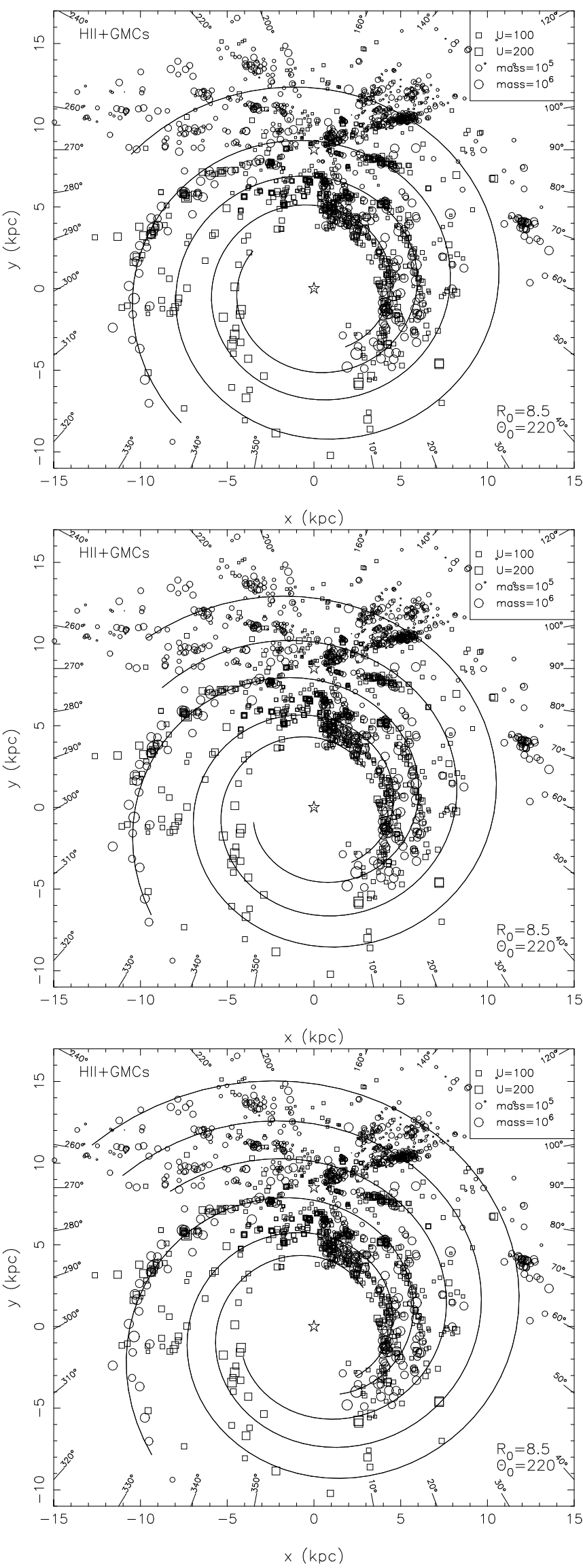

Fig. 5. The distribution of HII regions and GMCs $\left(R_{0}=8.5 \mathrm{kpc}\right.$ and $\Theta_{0}=220 \mathrm{~km} \mathrm{~s}^{-1}$ ) and the best-fitting two-arm model (top panel), threearm model (middle panel) and four-arm model (bottom panel).
The best fitting two-arm logarithmic model places the Sun in the Carina arm, and it also connects the Local arm and the Perseus arm with the Carina arm, which is inconsistent with observations. In fact, the connection of the Sagittarius arm and the Carina arm as a single arm has been well established from observations. Such a connection cannot be obtained in any two-arm model, as found by R03. In addition, the tangential directions calculated from this model are also very different from observed values. The parameter $Z$ is slightly larger than that of the 3- and 4-arm models. The best two-arm logarithmic model can be excluded, conclusively.

In the best fitting 3-arm and 4-arm models, the Sagittarius arm and the Carina arm are connected and look very similar. The Scutum arm in the 1st Galactic quadrant outlined by HII regions and molecular clouds is connected to the Crux arm in the 4th Galactic quadrant. This arm is well fitted by both the 3 -arm and the 4-arm models. However, the obvious difference arises from the extension of the Crux arm. It connects to the Perseus +1 arm in the 3 -arm model, but to the Perseus +2 arm in the the 4-arm model. It is not clear from current available data whether there is one arm or two arms in the few kpc outside the Perseus arm. Conservatively, the 3 -arm model predicts only one Perseus +1 arm and no Perseus +2 arm, which may be more realistic. However, Levine et al. (2006) showed that the HI gas distribution indicates that both the Perseus +1 arm and Perseus +2 arm are possible. The molecular clouds at $l \sim 70^{\circ}$ at $13 \mathrm{kpc}$ (Digel et al. 1990) may be related to the Perseus+2 arm. In the GC direction, the Norma arm is connected to the Perseus arm in the 3-arm model. In the 4-arm model, the Norma arm is connected to the Perseus $+1 \mathrm{arm}$, and the Perseus arm starts from another arm inside the Norma arm, corresponding to the Norma-Cygnus arm in the four-arm model of R03.

The tangential directions of arms in models are listed in Table 2 for comparison with observations. The double values of the Scutum tangent correspond to both the Scutum arm and another interior arm segment, which can be well fitted in our 3-arm and 4-arm models. However an obvious deviation is seen from the tangent of the actual Scutum arm $\left(l \sim 31^{\circ}\right.$ vs. $\left.l \sim 36^{\circ}\right)$. The tangent of the Sagittarius arm always deviates from observed values by $7^{\circ}$ or $4^{\circ}$ in the 3 -arm or the 4 -arm model. The tangent of the Norma arm differs by $4^{\circ}$ in the 4 -arm models.

\subsection{The polynomial logarithmic arm model}

Note that these spiral arm models are assumed to be purely logarithmic. However, the pitch angle of spiral arms can vary along the arm (see e.g. Seigar \& James 1998). If so, a polynomial logarithmic arm model may be necessary to fit both the distribution and tangential directions.

We first plotted the tracer data in the $\log (r)-\theta$ diagram (see Fig. 6), and roughly identified arm-like features and then separated the data by the dashed-lines. The Sagittarius-Carina arm is very prominent in the plot, which obviously separated in the tracer data from the other groups. The Scutum-Crux arm are also well-isolated in the tracer data, except for those at small $r$ where the data are mixed with those from the Sagittarius arm. The innermost arm seems clearly to have a group of tracers, which in fact is the Norma arm and connected to the Scutum arm. However, outside the Sagittarius-Carina arm, the data are well mixed, and one can barely distinguish the Perseus arm and the Perseus +1 arm, which can only be separated manually in a very rough manner.

The best model should satisfy the observed tangential directions and also minimize the parameter $Z$. We separate our total 
Table 2. Tangential directions from observations (Bronfman 1992; Englmaier \& Gerhard 1999) and the best fitting models.

\begin{tabular}{ccccccc}
\hline \hline Model & $\begin{array}{c}\text { Scutum } \\
\left({ }^{\circ}\right)\end{array}$ & $\begin{array}{c}\text { Sagittarius } \\
\left({ }^{\circ}\right)\end{array}$ & $\begin{array}{c}\text { Carina } \\
\left({ }^{\circ}\right)\end{array}$ & $\begin{array}{c}\text { Crux } \\
\left({ }^{\circ}\right)\end{array}$ & $\begin{array}{c}\text { Norma } \\
\left({ }^{\circ}\right)\end{array}$ & $\begin{array}{c}3-\mathrm{kpc} \\
\left({ }^{\circ}\right)\end{array}$ \\
\hline observed mean value & 25,31 & 51 & 284 & 310 & 327 & 339 \\
\hline For distribution of tracers with $R_{0}=8.5 \mathrm{kpc}$ and & $\Theta_{0}=220 \mathrm{~km} \mathrm{~s}^{-1}$ \\
2-arm model & 33 & 49 & 301 & 319 & 330 \\
3-arm model & 26,36 & 58 & 284 & 313 & 325 & \\
4-arm model & 25,36 & 55 & 283 & 311 & 323 & \\
polynomial model & 27,33 & 53 & 284 & 309 & 329 & \\
\hline For distribution of tracers with $R_{0}=8.0 \mathrm{kpc}$ and $\Theta_{0}=220 \mathrm{~km} \mathrm{~s}^{-1}$ \\
2-arm model & 33 & 49 & & 303 & 320 & 330 \\
3-arm model & 26,38 & 59 & 282 & 311 & 324 & \\
4-arm model & 26,37 & 55 & 284 & 310 & 322 & \\
polynomial model & 27,34 & 53 & 284 & 309 & 329 & \\
\hline
\end{tabular}

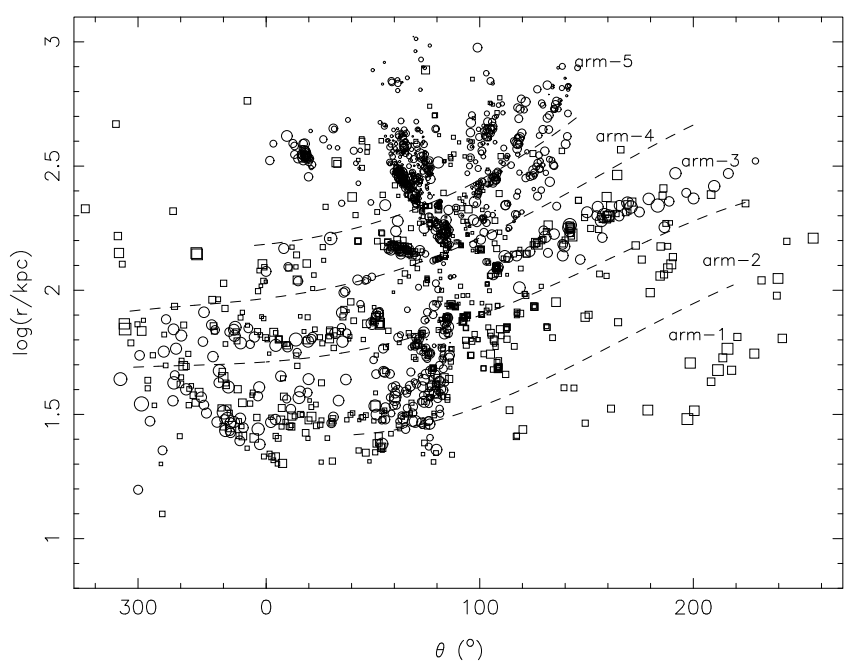

Fig. 6. The distribution of tracer data $\left(R_{0}=8.5 \mathrm{kpc}\right.$ and $\Theta_{0}=$ $220 \mathrm{~km} \mathrm{~s}^{-1}$ ) plotted in the $\log (r)-\theta$ diagram. Here $\theta$ starts at the positive $x$-axis and increase counterclockwise. The dash lines roughly separate main arm tracers.

sample into several sub-samples for five arms (see Fig. 6), and fit them separately with the polynomial logarithmic arm model:

$$
\ln r=a_{i}+b_{i} \theta+c_{i} \theta^{2}+d_{i} \theta^{3}
$$

to obtain the initial values of each arm, and finally we fit all arms together to the whole sample of data. After using the weighting factors, and minimizing the residual value by Eq. (6), we obtained the best model with the fitting parameters listed in Table 3 . The data and arms are plotted in Fig. 7. The tangential directions of this model are also listed in Table 2.

In this model, almost all tracers are best connected with the outlined spiral arms. Compared to the pure logarithmic models, the polynomial logarithmic arm model is more conservative in connecting known tracers. It does not outline the possible arm trajectory in the regions without tracers and predict the possible connection between the outer arms and inner arms. As seen in Fig. 7, the main spiral structures are well traced. From the center we find arm- 1 to arm-5, which correspond to the Norma arm, the Scutum-Crux arm, the Sagittarius-Carina arm, the Perseus arm and the Perseus +1 arm. Looking at the $\mathrm{HI}$ data in Fig. 4, one may see that arm-5, i.e. the Perseus +1 arm, seems to be connected to the obvious outer HI arm outlined by
Table 3. The parameters of the polynomial logarithmic arm model.

\begin{tabular}{|c|c|c|c|c|c|}
\hline$i$ th arm & $a_{i}$ & $b_{i}$ & $c_{i}$ & $d_{i}$ & $\theta_{i}$ \\
\hline \multicolumn{5}{|c|}{ For distribution with $R_{0}=8.5 \mathrm{kpc}$ and $\Theta_{0}=220 \mathrm{~km} \mathrm{~s}^{-1}$} & $\left({ }^{\circ}\right)$ \\
\hline arm-1 & 1.376 & -0.07792 & 0.04309 & 0 & 41 \\
\hline arm-2 & 7.330 & -2.302 & 0.2849 & -0.01059 & 304 \\
\hline arm-3 & 10.403 & -3.526 & 0.4620 & -0.01895 & 298 \\
\hline arm-4 & 1.978 & -0.1181 & 0.02098 & 0 & 338 \\
\hline arm-5 & 2.297 & 0.09116 & 0.04273 & 0 & 35 \\
\hline$Z$ & 0.31 & & & & \\
\hline \multicolumn{5}{|c|}{ For distribution with $R_{0}=8.0 \mathrm{kpc}$ and $\Theta_{0}=220 \mathrm{~km} \mathrm{~s}^{-1}$} & $\left({ }^{\circ}\right)$ \\
\hline arm-1 & 1.302 & -0.06629 & 0.04115 & 0 & 47 \\
\hline arm-2 & 7.5616 & -2.448 & 0.3090 & -0.01187 & 304 \\
\hline arm-3 & 9.9843 & -3.417 & 0.4522 & -0.01873 & 298 \\
\hline arm-4 & 1.5189 & -0.00581 & 0.01286 & 0 & 338 \\
\hline arm-5 & 2.1949 & 0.1037 & 0.03317 & 0 & 35 \\
\hline$Z$ & 0.26 & & & & \\
\hline \multicolumn{5}{|c|}{ For distribution with $R_{0}=8.4 \mathrm{kpc}$ and $\Theta_{0}=254 \mathrm{~km} \mathrm{~s}^{-1}$} & $\left({ }^{\circ}\right)$ \\
\hline arm-1 & 1.3747 & -0.0737 & 0.04318 & 0 & 41 \\
\hline arm-2 & 11.2880 & -3.8678 & 0.4900 & -0.01949 & 304 \\
\hline arm-3 & 10.2992 & -3.519 & 0.4662 & -0.01937 & 298 \\
\hline arm-4 & 1.5645 & -0.005055 & 0.0132 & 0 & 338 \\
\hline arm-5 & 2.2973 & 0.004478 & 0.0750 & 0 & 55 \\
\hline Z & 0.29 & & & & \\
\hline
\end{tabular}

Levine et al. (2006) at the both ends at about $(x, y)=(-12 \mathrm{kpc}$, $+12 \mathrm{kpc})$ and $(+8 \mathrm{kpc},+10 \mathrm{kpc})$. The Perseus arm is probably connected to the long $\mathrm{HI}$ arm at $(-12 \mathrm{kpc},+3 \mathrm{kpc})$. The tangential directions of this model (Table 2) are more consistent with the observed values than those from the logarithmic arm model. We conclude that such a polynomial arm model is a better description of the observed arm features.

The pitch angles of spiral arms vary in the form of $\tan \left(\psi_{i}\right)=$ $b_{i}+2 c_{i} \theta+3 d_{i} \theta^{2}$, in contrast to the constant pitch angles for the pure logarithmic arms. The pitch angles vary with azimuthal angle, as shown in Fig. 8. Variable pitch angles have been found in nearby galaxies. For example, Puerari \& Dottori (1992) used a simple model with a variable pitch angle to obtain the leading or trailing character of the arms of some galaxies. Note, however, that the innermost segments of arm- $2\left(l<\sim 25^{\circ}\right)$ and arm-3 $\left(l<\sim 35^{\circ}\right.$ ), probably due to the distance uncertainty of tracers, show unreasonable negative pitch angles in Fig. 8, similar to the possible outer HI arm $(x \sim 10 \mathrm{kpc})$ in the 1st quadrant as shown in Fig. 4. This is obtained from tracer data, which thus needs to be resolved. 

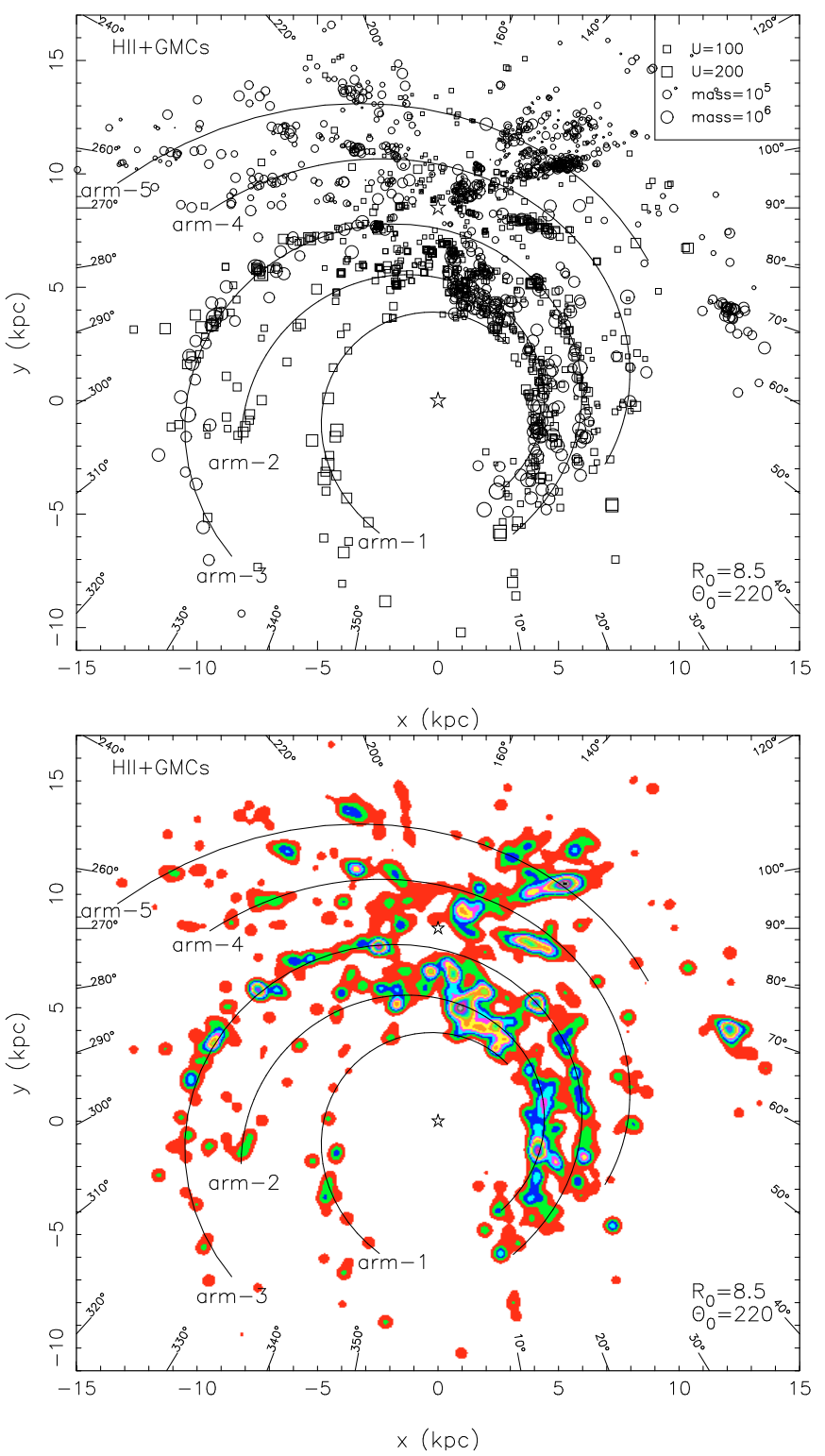

Fig. 7. The best-fitting polynomial logarithmic-arm model $\left(R_{0}=\right.$ $8.5 \mathrm{kpc}$ and $\Theta_{0}=220 \mathrm{~km} \mathrm{~s}^{-1}$ ), plotted onto the data distribution (top panel) and the color brightened-tracer image (bottom panel) of tracers. Almost all main tracers are connected by the outlined arms, except for one complex at $(x, y)=(12 \mathrm{kpc}, 4 \mathrm{kpc})$ which either has an overestimated distance or it belongs to another outer arm.

\subsection{The influence of the solar parameters $R_{0}$ and $\Theta_{0}$}

We also have re-estimated the distances of HII regions and GMCs, if not available from other measurements, by using the rotation curve for $R_{0}=8.0 \mathrm{kpc}$ and $\Theta_{0}=220 \mathrm{~km} \mathrm{~s}^{-1}$. Then the excitation parameters of HII regions and the masses of GMCs are accordingly recalculated, as listed in Tables A.1 and A.2. The final distribution of the tracers is shown in Fig. 9. The overall design is similar to that of $R_{0}=8.5 \mathrm{kpc}$ and $\Theta_{0}=220 \mathrm{~km} \mathrm{~s}^{-1}$. We also fit the distribution with two, three, and four spiral-arm models, and the parameter values from the best models are listed in Table 1. The best fit of a polynomial logarithmic arm model for a spiral structure is presented in Fig. 9, and the parameters are listed in Table 3. The number of arms and arm structure are conserved. As occured for the case of $8.5 / 220$, the tangential

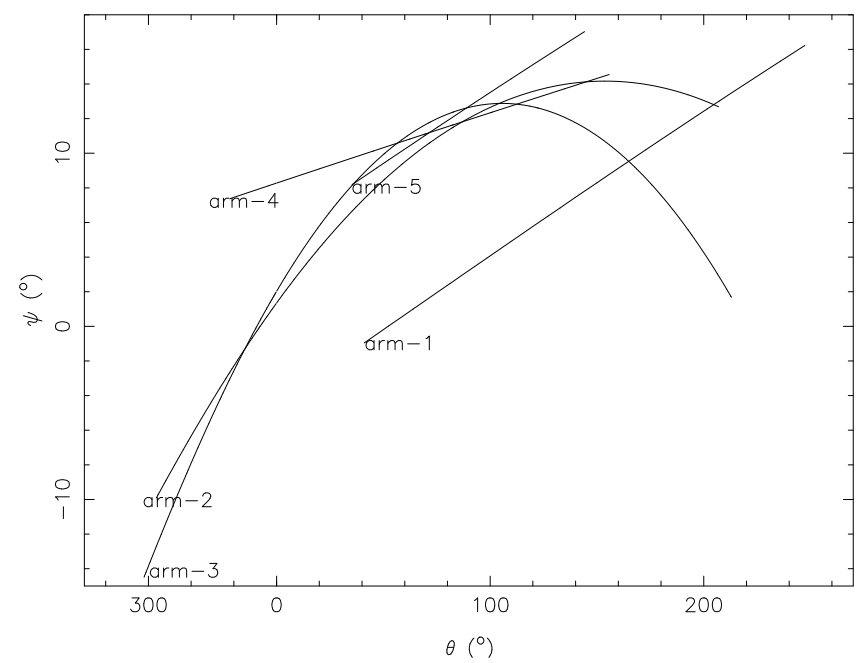

Fig. 8. The pitch angle $(\psi)$ variation of spiral arms in the accepted polynomial logarithmic-arm model (with $R_{0}=8.5 \mathrm{kpc}$ and $\Theta_{0}=$ $220 \mathrm{~km} \mathrm{~s}^{-1}$ ).

directions are better reproduced by the polynomial model than the logarithmic spiral arm models.

Reid et al. (2009) used the newly measured trigonometric parallaxes of masers to estimate the solar parameters, and concluded that $R_{0}=8.4 \pm 0.6 \mathrm{kpc}$ and $\Theta_{0}=254 \pm 16 \mathrm{~km} \mathrm{~s}^{-1}$. They offer a revised prescription (and a Fortran code) to calculate kinematic distances and their uncertainties (see Reid et al. 2009 , for details). Using the code they kindly provided to us, we determined the kinematic distances and re-scaled other parameters of our HII regions and GMC samples. Their distribution and the best-fitted polynomial logarithmic-arm model is shown in Fig. 10. Compared with the distribution with $R_{0}=8.5 \mathrm{kpc}$ and $\Theta_{0}=220 \mathrm{~km} \mathrm{~s}^{-1}$, the tracers are nearer to our sun in the second and third Galactic quadrants, so that arms in this region are wound more tightly. The local arm becomes clearer, but the separation between the Perseus arm and Perseus+1 becomes less clear. The grand design of spiral arms in the other parts of the Galaxy looks very similar to that of 8.0/220.

\section{Conclusions and remarks}

We collected the spiral tracer data of HII regions and giant molecular clouds (GMCs) published in the literature, and used them to study the weighted distribution of these tracers for the overall design of spiral arms of our Milky Way Galaxy. The mass of the GMC and the excitation parameter of an HII region are scaled as the weighting factors. The distances of tracers, if not available from stellar or triangulation measurements, were estimated kinetically using the rotation curve of BB93 with solar constants of $R_{0}=8.5 \mathrm{kpc}$ and $\Theta_{0}=220 \mathrm{~km} \mathrm{~s}^{-1}$. We fitted the distribution of these tracers with two, three, and four logarithmic spiral arm models. None of the models can match all the main arm features observed. We finally fit the tracer data by using a polynomial logarithmic arm model, which not only can fit the distribution of tracers for the main spiral arms, but also match the tangential directions with the observations. The polynomial model appears to be better able to describe the spiral arms of our Galaxy. When the kinematic distances are estimated by using rotation curves with two sets of parameters, $R_{0}=8.0 \mathrm{kpc}$ and $\Theta_{0}=220 \mathrm{~km} \mathrm{~s}^{-1}$ and $R_{0}=8.4 \mathrm{kpc}$ and $\Theta_{0}=254 \mathrm{~km} \mathrm{~s}^{-1}$, the distribution of HII regions and GMCs are slightly better fitted by the models, though the parameters do not change significantly. 

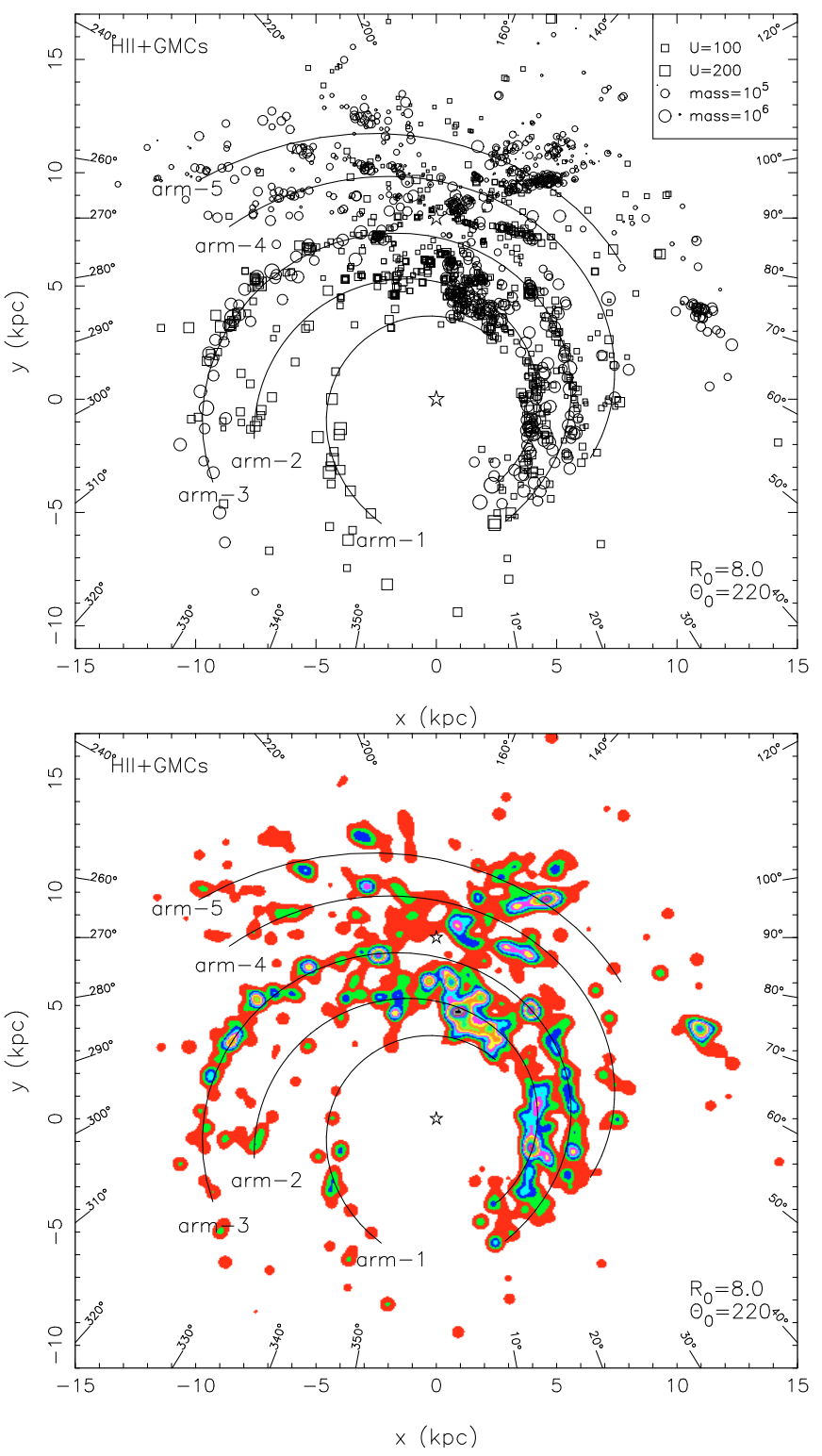

Fig. 9. The best-fitting polynomial logarithmic-arm model for $R_{0}=$ $8.0 \mathrm{kpc}$ and $\Theta_{0}=220 \mathrm{~km} \mathrm{~s}^{-1}$, plotted on the data distribution (top panel) and the color brightened-tracer image (bottom panel) of tracers.

For most of tracers in our work, we use their kinematic distances derived from the rotation curves. Gómez (2006) found that the error bars are significantly large for objects at the positions of spiral arms. This pitfall cannot be overcome until accurate distances of a large sample of HII regions are measured, e.g. by triangulation of radio masers (e.g. Xu et al. 2006; Reid et al. 2009). A large project is being carried out with the VLBA to directly measure distances of a large sample of massive star formation regions by trigonometric parallaxes (see Reid et al. 2008 ) with accuracies approaching $\sim 10 \mu$ as. Published results of several examples show systematically smaller distances than the kinematic distances. They will probably obtain hundreds of measurements over 10 years for the brightest HII regions covering all the Galactic disk, and the final grand design of our Milky Way will be greatly improved. However, at present, the picture shown in Fig. 10 is the best approximate description of Galactic spiral structure.

Acknowledgements. We thank the referee for instructive comments, and Dr. Mark Reid for providing the Fortran code for the estimation of kinematic
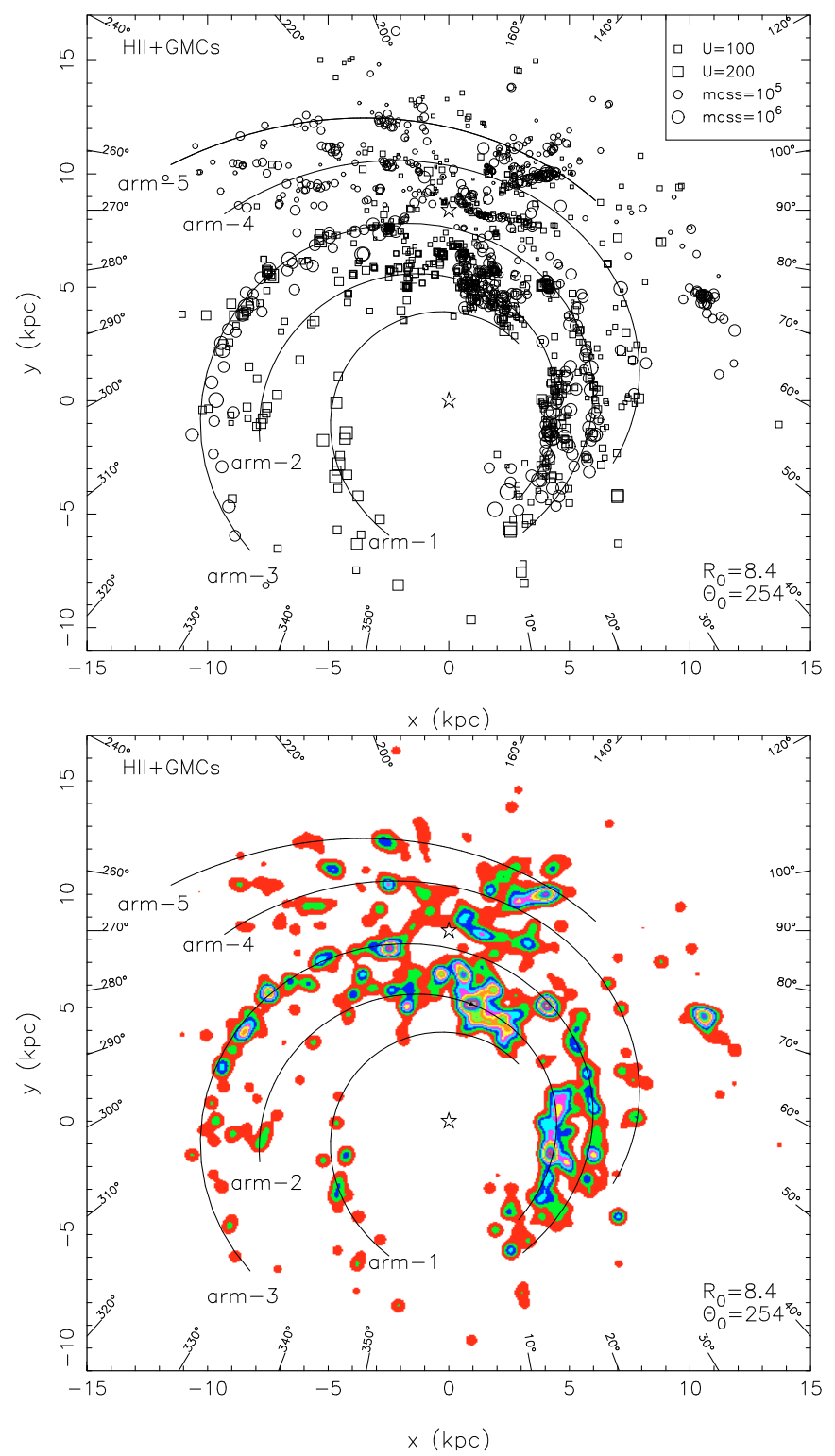

Fig. 10. The same as Fig. 9 but with the rotation curve from Reid et al. (2009) with $R_{0}=8.4 \mathrm{kpc}$ and $\Theta_{0}=254 \mathrm{~km} \mathrm{~s}^{-1}$.

distances using their newly determined rotation curves. The authors are supported by the National Natural Science Foundation (NNSF) of China (10773016, 10821061 and 10833003) and the National Key Basic Research Science Foundation of China (2007CB815403).

\section{References}

Anderson, L. D., \& Bania, T. M. 2009, ApJ, 690, 706

Araya, E., Hofner, P., Churchwell, E., \& Kurtz, S. 2002, ApJS, 138, 63

Blum, R. D., Damineli, A., \& Conti, P. S. 2001, AJ, 121, 3149

Brand, J., \& Blitz, L. 1993, A\&A, 275, 67

Brand, J., \& Wouterloot, J. G. A. 1994, A\&AS, 103, 503

Bronfman, L. 1992, in The center, bulge, and disk of the Milky Way, ed. L. Blitz, Astrophys. Space Sci. Libr., 180, 131

Brunthaler, A., Reid, M. J., Menten, K. M., et al. 2008, ApJ, submitted [arXiv: 0811.0713]

Carpenter, J. M., Snell, R. L., \& Schloerb, F. P. 1990, ApJ, 362, 147

Caswell, J. L., \& Haynes, R. F. 1987, A\&A, 171, 261

Clemens, D. P. 1985, ApJ, 295, 422

Cohen, R. S., Dame, T. M., \& Thaddeus, P. 1986, ApJS, 60, 695

Condon, J. J., Cotton, W. D., Greisen, E. W., et al. 1998, AJ, 115, 1693

Copetti, M. V. F., Oliveira, V. A., Riffel, R., Castañeda, H. O., \& Sanmartim, D. 2007, A\&A, 472, 847 
Crowther, P. A., \& Furness, J. P. 2008, A\&A, 492, 111

Dame, T. M., Elmegreen, B. G., Cohen, R. S., \& Thaddeus, P. 1986, ApJ, 305, 892

Dame, T. M., Hartmann, D., \& Thaddeus, P. 2001, ApJ, 547, 792

Dawson, J. R., Kawamura, A., Mizuno, N., Onishi, T., \& Fukui, Y. 2008, PASJ, 60, 1297

Digel, S., Thaddeus, P., \& Bally, J. 1990, ApJ, 357, L29

Digel, S., de Geus, E., \& Thaddeus, P. 1994, ApJ, 422, 92

Digel, S. W., Lyder, D. A., Philbrick, A. J., Puche, D., \& Thaddeus, P. 1996, ApJ, 458,561

Downes, D., Wilson, T. L., Bieging, J., \& Wink, J. 1980, A\&AS, 40, 379

Eisenhauer, F., Schödel, R., Genzel, R., et al. 2003, ApJ, 597, L121

Englmaier, P., \& Gerhard, O. 1999, MNRAS, 304, 512

Fich, M., Blitz, L., \& Stark, A. A. 1989, ApJ, 342, 272

Fish, V. L., Reid, M. J., Wilner, D. J., \& Churchwell, E. 2003, ApJ, 587, 701

Georgelin, Y. M., \& Georgelin, Y. P. 1976, A\&A, 49, 57

Ghez, A. M., Salim, S., Weinberg, N. N., et al. 2008, ApJ, 689, 1044

Gillessen, S., Eisenhauer, F., Trippe, S., et al. 2009, ApJ, 692, 1075

Gómez, G. C. 2006, AJ, 132, 2376

Grabelsky, D. A., Cohen, R. S., Bronfman, L., \& Thaddeus, P. 1988, ApJ, 331, 181

Gregory, P. C., Scott, W. K., Douglas, K., \& Condon, J. J. 1996, ApJS, 103, 427 Griffith, M. R., Wright, A. E., Burke, B. F., \& Ekers, R. D. 1994, ApJS, 90, 179 Griffith, M. R., Wright, A. E., Burke, B. F., \& Ekers, R. D. 1995, ApJS, 97, 347 Groenewegen, M. A. T., Udalski, A., \& Bono, G. 2008, A\&A, 481, 441

Heyer, M. H., Carpenter, J. M., \& Snell, R. L. 2001, ApJ, 551, 852

Kolpak, M. A., Jackson, J. M., Bania, T. M., Clemens, D. P., \& Dickey, J. M 2003, ApJ, 582, 756

Kuchar, T. A., \& Bania, T. M. 1994, ApJ, 436, 117

Leahy, D. A., \& Tian, W. W. 2008, AJ, 135, 167

Levine, E. S., Blitz, L., \& Heiles, C. 2006, Science, 312, 1773

May, J., Alvarez, H., \& Bronfman, L. 1997, A\&A, 327, 325

Mead, K. N., \& Kutner, M. L. 1988, ApJ, 330, 399
Moscadelli, L., Reid, M. J., Menten, K. M., et al. 2008, ApJ, submitted [arXiv: 0811.0679]

Nakagawa, M., Onishi, T., Mizuno, A., \& Fukui, Y. 2005, PASJ, 57, 917

Naoz, S., \& Shaviv, N. J. 2007, New Astron., 12, 410

Paladini, R., Burigana, C., Davies, R. D., et al. 2003, A\&A, 397, 213

Paladini, R., Davies, R. D., \& DeZotti, G. 2004, MNRAS, 347, 237

Pandian, J. D., Momjian, E., \& Goldsmith, P. F. 2008, A\&A, 486, 191

Puerari, I., \& Dottori, H. A. 1992, A\&AS, 93, 469

Reid, M. J. 1993, ARA\&A, 31, 345

Reid, M. J., Menten, K. M., Brunthaler, A., et al. 2008, ApJ, submitted [arXiv: 0811.0595]

Reid, M. J., Menten, K. M., Zheng, X. W., et al. 2009, ApJ, submitted [arXiv: 0902 . 3913]

Russeil, D. 2003, A\&A, 397, 133

Russeil, D., Adami, C., \& Georgelin, Y. M. 2007, A\&A, 470, 161

Schraml, J., \& Mezger, P. G. 1969, ApJ, 156, 269

Seigar, M. S., \& James, P. A. 1998, MNRAS, 299, 685

Sewilo, M., Watson, C., Araya, E., et al. 2004, ApJS, 154, 553

Sodroski, T. J. 1991, ApJ, 366, 95

Solomon, P. M., Rivolo, A. R., Barrett, J., \& Yahil, A. 1987, ApJ, 319, 730

Tian, W. W., \& Leahy, D. A. 2008, ApJ, 677, 292

Tian, W. W., Li, Z., Leahy, D. A., \& Wang, Q. D. 2007, ApJ, 657, L25

Tian, W. W., Leahy, D. A., Haverkorn, M., \& Jiang, B. 2008, ApJ, 679, L85

Vallée, J. P. 2008, AJ, 135, 1301

Watson, C., Araya, E., Sewilo, M., et al. 2003, ApJ, 587, 714

Wright, A. E., Griffith, M. R., Burke, B. F., \& Ekers, R. D. 1994, ApJS, 91,111

Wright, A. E., Griffith, M. R., Hunt, A. J., et al. 1996, ApJS, 103, 145

Xu, Y., Reid, M. J., Zheng, X. W., \& Menten, K. M. 2006, Science, 311, 54

Xu, Y., Reid, M. J., Menten, K. M., et al. 2008, ApJ, submitted [arXiv:0811.0701]

Zhang, B., Zheng, X. W., Reid, M. J., et al. 2008, ApJ, submitted [arXiv:0811.0704] 
L. G. Hou et al.: The spiral structure of our Milky Way Galaxy, Online Material $p 1$

\section{Appendix A: Tracer data for spiral structure}

We collected and calculated the related parameters of HII regions and giant molecular clouds (GMCs) based on the observations in the literature, and present them here in two tables. 


\section{G. Hou et al.: The spiral structure of our Milky Way Galaxy, Online Material $p 2$}

Table A.1. HII regions as tracers of spiral arms. Columns 1 to 3 list the Galactic longitude, Galactic latitude and velocity, taken from the reference given in Col. 4; Cols. 5 to 7 give the radio continuum flux of the HII region, observation frequency and the reference. Columns 8 to 10 give the stellar distance when available, as well as the error and the reference; Col. 11 is a note for the distance ambiguity and Col. 12 is its reference: Knear - the nearer kinematic distance is adopted; Kfar - the farther kinematic distance is adopted; Ktan - the tracer is located at the tangential point and the distance to the tangent is adopted; Kout - the object is in the outer Galaxy and only one kinematic distance is available and adopted; Cols. 13 and 14 give the kinematic distance to the Sun and distance to the Galactic Center estimated with a rotation curve with $R_{0}=8.5 \mathrm{kpc}$, $\Theta_{0}=220 \mathrm{~km} \mathrm{~s}^{-1}$; Col. 15 contains the excitation parameter rescaled by the distance we adopted; Cols. 16 to 18 are the same as Cols. 13-15 but with constants $R_{0}=8.0 \mathrm{kpc}$ and $\Theta_{0}=220 \mathrm{~km} \mathrm{~s}^{-1}$ for kinematic distances. Columns 19 to 21 are the same as Cols. 13-15 but with constants $R_{0}=8.4 \mathrm{kpc}$ and $\Theta_{0}=254 \mathrm{~km} \mathrm{~s}^{-1}$ for kinematic distances.

\begin{tabular}{|c|c|c|c|c|c|c|c|c|c|c|c|c|c|c|c|c|c|c|c|c|}
\hline $\begin{array}{r}l \\
\left({ }^{\circ}\right) \\
(1)\end{array}$ & $\begin{array}{r}b \\
\left({ }^{\circ}\right) \\
(2)\end{array}$ & $\begin{array}{c}V_{\mathrm{lsr}} \\
\mathrm{km} \mathrm{s}^{-1} \\
(3)\end{array}$ & Ref. & $\begin{array}{c}S \\
\text { Jy } \\
(5)\end{array}$ & $\begin{array}{r}\text { Freq } \\
\text { GHz } \\
(6)\end{array}$ & Ref. & $\begin{array}{r}d \\
\mathrm{kpc} \\
(8)\end{array}$ & $\begin{array}{r}d_{\text {erro }} \\
\text { kpc } \\
(9)\end{array}$ & (10) & Mark & $\begin{array}{l}\text { Ref. } \\
\text { (12) }\end{array}$ & $\begin{array}{l}D_{8.5} \\
\mathrm{kpc} \\
(13)\end{array}$ & $\begin{array}{l}R_{8.5} \\
\mathrm{kpc} \\
(14)\end{array}$ & $\begin{array}{r}U_{8.5} \\
\mathrm{pc} \mathrm{cm}^{-2} \\
(15)\end{array}$ & $\begin{array}{l}D_{8.0} \\
\mathrm{kpc} \\
(16)\end{array}$ & $\begin{array}{l}R_{8.0} \\
\mathrm{kpc} \\
(17)\end{array}$ & $\begin{array}{r}U_{8.0} \\
\mathrm{pc} \mathrm{cm}^{-2} \\
(18)\end{array}$ & $\begin{array}{l}D_{8.4} \\
\mathrm{kpc} \\
(19)\end{array}$ & $\begin{array}{l}R_{8.4} \\
\mathrm{kpc} \\
(20)\end{array}$ & $\begin{array}{r}U_{8.4} \\
\mathrm{pc} \mathrm{cm^{-2 }} \\
(21)\end{array}$ \\
\hline 2.40 & 1.40 & 3.0 & $\mathrm{pbd}+03$ & 3.00 & 2.70 & $\mathrm{pbd}+03$ & 4.7 & 1.4 & rus03 & & & & & 54.7 & & & 54.7 & & & 54.7 \\
\hline 2.90 & -0.00 & -1.7 & $\mathrm{pbd}+03$ & 3.40 & 2.70 & $\mathrm{pbd}+03$ & & & & kout & hh09 & 18.7 & 10.3 & 143.3 & 17.4 & 9.4 & 136.6 & 18.2 & 9.7 & 140.7 \\
\hline 4.30 & 0.60 & 6.6 & rus 03 & & & & 1.3 & 0.4 & rus 03 & & & & & 28.0 & & & 28.0 & & & 28.0 \\
\hline 6.00 & -1.30 & 2.8 & $\mathrm{pbd}+03$ & 44.90 & 2.70 & $\mathrm{pbd}+03$ & 1.6 & 0.25 & rus 03 & & & & & 65.8 & & & 65.8 & & & 65.8 \\
\hline 6.00 & -1.20 & 3.6 & $\mathrm{pbd}+03$ & 113.40 & 2.70 & $\mathrm{pbd}+03$ & 1.6 & 0.25 & rus03 & & & & & 89.5 & & & 89.5 & & & 89.5 \\
\hline 6.20 & -1.20 & 3.0 & $\mathrm{pbd}+03$ & 2.10 & 2.70 & $\mathrm{pbd}+03$ & 1.6 & 0.25 & rus03 & & & & & 23.7 & & & 23.7 & & & 23.7 \\
\hline 6.40 & -0.50 & 19.0 & $\mathrm{pbd}+03$ & 4.00 & 2.70 & $\mathrm{pbd}+03$ & & & & knear & pdd04 & 3.8 & 4.8 & 52.3 & 3.5 & 4.5 & 49.5 & 3.6 & 5.0 & 50.4 \\
\hline 6.50 & 0.10 & 14.1 & $\mathrm{pbd}+03$ & 0.50 & 2.70 & $\mathrm{pbd}+03$ & & & & knear & pdd04 & 3.1 & 5.4 & 22.8 & 2.9 & 5.1 & 21.8 & 2.9 & 5.6 & 21.8 \\
\hline 6.60 & -0.30 & 16.1 & $\mathrm{pbd}+03$ & 54.00 & 2.70 & $\mathrm{pbd}+03$ & & & & knear & pdd04 & 3.4 & 5.2 & 115.6 & 3.1 & 4.9 & 108.7 & 3.2 & 5.4 & 111.0 \\
\hline 6.60 & -0.10 & 13.4 & $\mathrm{pbd}+03$ & 11.10 & 2.70 & $\mathrm{pbd}+03$ & & & & knear & pdd04 & 3.0 & 5.5 & 62.7 & 2.8 & 5.2 & 59.9 & 2.8 & 5.7 & 59.9 \\
\hline 6.70 & -0.20 & 19.4 & $\mathrm{pbd}+03$ & 7.60 & 2.70 & $\mathrm{pbd}+03$ & & & & knear & pdd04 & 3.7 & 4.8 & 63.6 & 3.5 & 4.6 & 61.3 & 3.5 & 5.0 & 61.3 \\
\hline 6.90 & -2.10 & 5.4 & $\mathrm{pbd}+03$ & 15.40 & 2.70 & $\mathrm{pbd}+03$ & 1.6 & 0.25 & rus03 & & & & & 46.0 & & & 46.0 & & & 46.0 \\
\hline 7.00 & -0.20 & 14.3 & $\mathrm{pbd}+03$ & 15.00 & 2.70 & pbd +03 & 2.7 & 0.8 & rus03 & knear & pdd04 & 3.0 & 5.5 & 64.7 & & & 64.7 & & & 64.7 \\
\hline 7.30 & 0.10 & 17.9 & $\mathrm{pbd}+03$ & 1.70 & 2.70 & $\mathrm{pbd}+03$ & 2.7 & 0.8 & rus03 & & & & & 31.3 & & & 31.3 & & & 31.3 \\
\hline 8.40 & 0.30 & 37.9 & $\mathrm{pbd}+03$ & 2.60 & 2.70 & $\mathrm{pbd}+03$ & & & & knear & pdd04 & 4.8 & 3.8 & 52.9 & 4.4 & 3.7 & 49.9 & 4.5 & 4.1 & 50.7 \\
\hline 8.70 & -0.40 & 41.8 & $\mathrm{pbd}+03$ & 1.80 & 2.70 & $\mathrm{pbd}+03$ & & & & knear & pdd04 & 4.9 & 3.7 & 47.5 & 4.6 & 3.5 & 45.5 & 4.7 & 4.0 & 46.2 \\
\hline 8.70 & -0.50 & 15.0 & rus03 & & & & 1.7 & 0.5 & rus03 & & & & & & & & & & & \\
\hline 10.16 & -0.35 & 12.5 & swa+04 & 25.50 & 4.86 & swa+04 & & & & kfar & wa+04 & 14.6 & 6.4 & 242.5 & 13.8 & 6.1 & 233.6 & 14.5 & 6.3 & 241.4 \\
\hline 10.20 & -0.30 & 13.6 & $\mathrm{pbd}+03$ & 55.90 & 2.70 & $\mathrm{pbd}+03$ & & & & kfar & $\operatorname{gg} 76$ & 14.5 & 6.3 & 307.5 & 13.6 & 5.9 & 294.6 & 14.4 & 6.2 & 306.1 \\
\hline 10.31 & -0.15 & 9.4 & swa+04 & 8.70 & 4.86 & swa+04 & 3.4 & 0.3 & bdc01 & knear & pmg08 & 1.6 & 6.9 & 64.1 & & & 64.1 & & & 64.1 \\
\hline 10.46 & 0.03 & 70.2 & swa+04 & 1.07 & 4.86 & swa+04 & & & & kfar & swa+04 & 10.9 & 3.0 & 69.3 & 10.4 & 2.9 & 67.2 & 11.1 & 3.1 & 70.1 \\
\hline 10.60 & -0.40 & 0.2 & $\mathrm{pbd}+03$ & 10.50 & 2.70 & $\mathrm{pbd}+03$ & & & & kout & hh09 & 16.8 & 8.6 & 194.2 & & & & 16.3 & 8.1 & 190.3 \\
\hline 10.70 & 0.50 & -2.4 & $\mathrm{dd}+03$ & 2.50 & 2.70 & $\mathrm{pbd}+03$ & & & & kout & hh09 & 17.4 & 9.2 & 123.2 & 16.2 & 8.5 & 117.5 & 16.8 & 8.6 & 120.4 \\
\hline 10.96 & 0.02 & 17.7 & swa+04 & 0.98 & 4.86 & swa+04 & & & & kfar & swa+04 & 14.1 & 6.0 & 79.9 & 13.3 & 5.6 & 76.8 & 14.0 & 5.9 & 79.5 \\
\hline 11.11 & -0.40 & 2.1 & swa+04 & 0.69 & 4.86 & swa+04 & & & & kfar & swa+04 & 16.4 & 8.2 & 78.7 & 15.3 & 7.6 & 75.1 & 16.0 & 7.8 & 77.4 \\
\hline 11.50 & -1.00 & 12.0 & rus03 & 3.20 & 2.70 & $\mathrm{pbd}+03$ & 1.6 & 0.2 & rus03 & knear & pdd04 & 2.5 & 6.0 & 27.3 & & & 27.3 & & & 27.3 \\
\hline 11.93 & -0.61 & 40.3 & swa+04 & 1.64 & 4.86 & swa+04 & & & & knear & swa+04 & 4.2 & 4.5 & 42.3 & 3.9 & 4.2 & 40.3 & 4.0 & 4.7 & 40.9 \\
\hline 11.99 & -0.27 & 58.2 & pmg08 & 0.07 & 1.40 & $\operatorname{ccg}+98$ & & & & kfar & pmg08 & 11.5 & 3.7 & 27.8 & 10.9 & 3.5 & 26.8 & 11.6 & 3.8 & 28.0 \\
\hline 12.40 & -0.00 & -18.4 & $\mathrm{pbd}+03$ & 0.80 & 2.70 & $\mathrm{pbd}+03$ & & & & kout & hh09 & 22.6 & 14.4 & 100.3 & 20.8 & 13.1 & 94.9 & 20.9 & 12.7 & 95.2 \\
\hline 12.40 & -1.10 & 41.0 & $\mathrm{pbd}+03$ & 1.00 & 2.70 & $\mathrm{pbd}+03$ & & & & knear & pdd04 & 4.2 & 4.5 & 35.2 & 3.9 & 4.3 & 33.5 & 4.0 & 4.7 & 34.1 \\
\hline 12.41 & 0.51 & 34.1 & swa+04 & 0.11 & 4.86 & swa+04 & & & & knear & swa+04 & 3.7 & 4.9 & 15.8 & 3.5 & 4.7 & 15.2 & 3.5 & 5.1 & 15.2 \\
\hline 12.63 & -0.02 & 33.5 & swa+04 & 0.50 & 4.86 & swa+04 & & & & kfar & swa+04 & 12.9 & 5.0 & 60.2 & 12.2 & 4.7 & 58.0 & 12.9 & 5.0 & 60.2 \\
\hline 12.68 & -0.18 & 57.4 & pmg08 & 0.32 & 1.40 & $\operatorname{ccg}+98$ & & & & knear & pmg08 & 5.0 & 3.8 & 26.5 & 4.6 & 3.7 & 25.1 & 4.7 & 4.1 & 25.4 \\
\hline 12.78 & 0.33 & 15.8 & swa+04 & 0.37 & 4.86 & swa +04 & & & & kfar & swa+04 & 14.5 & 6.4 & 58.9 & 13.6 & 6.0 & 56.4 & 14.3 & 6.3 & 58.4 \\
\hline 12.80 & -0.20 & 36.0 & $\mathrm{pbd}+03$ & 41.70 & 2.70 & $\mathrm{pbd}+03$ & & & & knear & $\operatorname{gg} 76$ & 3.8 & 4.9 & 114.2 & 3.6 & 4.6 & 110.2 & 3.6 & 5.1 & 110.2 \\
\hline 12.80 & 0.40 & 20.7 & $\mathrm{pbd}+03$ & 4.10 & 2.70 & $\mathrm{pbd}+03$ & 2.4 & 0.2 & rus03 & & & & & 38.8 & & & 38.8 & & & 38.8 \\
\hline .91 & -0.26 & 31.7 & $a+04$ & 1.06 & 4.86 & swa+04 & & & & kfar & swa+04 & 13.1 & 5.1 & 78.1 & 12.3 & 4.9 & 74.9 & 13.1 & 5.1 & 78.1 \\
\hline 13.13 & -0.15 & 40.6 & $a+04$ & 0.24 & 4.86 & swa+04 & & & & kfar & swa+04 & 12.5 & 4.7 & 46.2 & 11.8 & 4.4 & 44.5 & 12.5 & 4.7 & 46.2 \\
\hline .20 & 0.00 & 55.1 & $d+03$ & 7.00 & 2.70 & $\mathrm{pbd}+03$ & & & & knear & gg76 & 4.8 & 4.0 & 73.6 & 4.4 & 3.8 & 69.5 & 4.5 & 4.2 & 70.5 \\
\hline 13.40 & 0.10 & 18.3 & $d+03$ & 10.20 & 2.70 & $\mathrm{pbd}+03$ & & & & kfar & pdd04 & 14.2 & 6.3 & 172.0 & 13.4 & 5.9 & 165.5 & 14.1 & 6.2 & 171.2 \\
\hline 50 & -0.20 & 11.3 & 03 & 60 & 2.70 & $\mathrm{pbd}+03$ & 1.6 & 0.25 & rus03 & & & & & 15.6 & & & 15.6 & & & 15.6 \\
\hline & -0.80 & 23.9 & +03 & .20 & 2.70 & $\mathrm{pbd}+03$ & 1.6 & 0.25 & rus03 & & & & & 44.8 & & & 44.8 & & & 44.8 \\
\hline 88 & 0.28 & 51.0 & +04 & & 4.86 & swa+04 & & & & knear & swa+04 & 4.5 & 4.3 & 60.4 & 4.2 & 4.1 & 57.7 & 4.2 & 4.5 & 57.7 \\
\hline .00 & -0.10 & 35.7 & $\mathrm{pb}$ & & 2.70 & $\mathrm{pbd}+03$ & & & & knear & pdd04 & 3.6 & 5.1 & 47.8 & 3.4 & 4.8 & 46.0 & 3.4 & 5.2 & 46.0 \\
\hline .09 & 0.10 & 16.3 & g08 & 0.11 & 1.40 & $\operatorname{ccg}+98$ & & & & kfar & pmg08 & 14.5 & 6.6 & 37.7 & 13.6 & 6.1 & 36.1 & 14.3 & 6.4 & 37.4 \\
\hline .10 & -0.50 & 20.6 & $d+03$ & 1.70 & 2.70 & $\mathrm{pbd}+03$ & 1.6 & 0.25 & rus03 & & & & & 22.1 & & & 22.1 & & & 22.1 \\
\hline 20 & 0.20 & 36.9 & $d+03$ & 28.80 & 2.70 & $\mathrm{pbd}+03$ & & & & knear & pdd04 & 3.7 & 5.0 & 99.2 & 3.4 & 4.8 & 93.8 & 3.5 & 5.2 & 95.6 \\
\hline .20 & -0.10 & 36.1 & $d+03$ & 1.90 & 2.70 & $\mathrm{pbd}+03$ & & & & knear & pdd04 & 3.6 & 5.1 & 39.3 & 3.4 & 4.8 & 37.8 & 3.4 & 5.2 & 37.8 \\
\hline .33 & -0.64 & 27.6 & $a+04$ & 0.39 & 4.86 & swa+04 & & & & knear & swa+04 & 3.0 & 5.6 & 21.0 & 2.8 & 5.3 & 20.1 & 2.9 & 5.8 & 20.5 \\
\hline 14.40 & -0.70 & 11.9 & $\mathrm{pbd}+03$ & 2.90 & 2.70 & $\mathrm{pbd}+03$ & 1.6 & 0.25 & rus03 & knear & pdd04 & 1.5 & 7.0 & 26.4 & & & 26.4 & & & 26.4 \\
\hline 14.60 & 0.00 & 34.9 & $\mathrm{pbd}+03$ & 40.00 & 2.70 & $\mathrm{pbd}+03$ & & & & knear & pdd04 & 3.5 & 5.2 & 106.6 & 3.3 & 4.9 & 102.5 & 3.3 & 5.4 & 102.5 \\
\hline 14.60 & 0.10 & 37.3 & $\mathrm{pbd}+03$ & 32.00 & 2.70 & $\mathrm{pbd}+03$ & & & & knear & pdd04 & 3.6 & 5.0 & 100.8 & 3.4 & 4.8 & 97.0 & 3.5 & 5.2 & 98.9 \\
\hline 14.68 & -0.50 & 27.5 & swa+04 & 0.49 & 4.86 & swa+04 & & & & kfar & swa+04 & 13.5 & 5.7 & 61.6 & 12.7 & 5.4 & 59.1 & 13.4 & 5.6 & 61.3 \\
\hline 15.00 & -0.70 & 13.9 & $\mathrm{pbd}+03$ & 489.00 & 2.70 & $\mathrm{pbd}+03$ & 1.6 & 0.25 & rus03 & knear & pdd04 & 1.7 & 6.9 & 145.7 & & & 145.7 & & & 145.7 \\
\hline
\end{tabular}


Table A.1. continued.

\begin{tabular}{|c|c|c|c|c|c|c|c|c|c|c|c|c|c|c|c|c|c|c|c|c|}
\hline $\begin{array}{r}l \\
\left({ }^{\circ}\right) \\
(1)\end{array}$ & $\begin{array}{r}b \\
\left({ }^{\circ}\right) \\
(2)\end{array}$ & $\begin{array}{c}V_{\mathrm{lsr}} \\
\mathrm{km} \mathrm{s}^{-1} \\
(3)\end{array}$ & Ref. & $\begin{array}{c}S \\
\text { Jy } \\
(5)\end{array}$ & $\begin{array}{r}\text { Freq } \\
\text { GHz } \\
(6)\end{array}$ & Ref. & $\begin{array}{r}d \\
\mathrm{kpc} \\
(8)\end{array}$ & $\begin{array}{r}d_{\text {erro }} \\
\text { kpc } \\
(9)\end{array}$ & (10) & Mark & Ref. & $\begin{array}{l}D_{8.5} \\
\mathrm{kpc} \\
(13)\end{array}$ & $\begin{array}{c}R_{8.5} \\
\mathrm{kpc} \\
(14)\end{array}$ & $\begin{array}{r}U_{8.5} \\
\mathrm{pc} \mathrm{cm}^{-2} \\
(15)\end{array}$ & $\begin{array}{l}D_{8.0} \\
\mathrm{kpc} \\
(16)\end{array}$ & $\begin{array}{l}R_{8.0} \\
\mathrm{kpc} \\
(17)\end{array}$ & $\begin{array}{r}U_{8.0} \\
\mathrm{pc} \mathrm{cm}^{-2} \\
(18)\end{array}$ & $\begin{array}{c}D_{8.4} \\
\mathrm{kpc} \\
(19)\end{array}$ & $\begin{array}{l}R_{8.4} \\
\mathrm{kpc} \\
(20)\end{array}$ & $\begin{array}{r}U_{8.4} \\
\mathrm{pc} \mathrm{cm}^{-2} \\
(21)\end{array}$ \\
\hline 15.10 & -0.90 & $\begin{array}{l}3.8 \\
\end{array}$ & $\mathrm{pbd}+03$ & 4.20 & 2.70 & $\mathrm{pbd}+03$ & 1.6 & 0.25 & $\begin{array}{ll}\text { rus03 } \\
\end{array}$ & knear & pdd04 & 1.7 & 6.9 & 29.8 & & & 29.8 & & & 29.8 \\
\hline 15.10 & -0.70 & 19.1 & $\mathrm{pbd}+03$ & 552.00 & 2.70 & $\mathrm{pbd}+03$ & 1.6 & 0.25 & rus03 & knear & pdd04 & 2.2 & 6.4 & 151.8 & & & 151.8 & & & 151.8 \\
\hline 15.20 & .60 & 13.9 & $\mathrm{bd}+03$ & 5.60 & 2.70 & $\mathrm{pbd}+03$ & 1.6 & 0.25 & rus03 & knear & pdd04 & 1.7 & 6.9 & 32.9 & & & 32.9 & & & 32.9 \\
\hline 15.20 & 0.80 & 23.7 & $\mathrm{pbd}+03$ & 7.10 & 2.70 & $\mathrm{pbd}+03$ & 1.6 & 0.25 & rus03 & knear & pdd04 & 2.6 & 6.0 & 35.6 & & & 35.6 & & & 35.6 \\
\hline 15.20 & 1.50 & 17.5 & rus03 & & & & 2.8 & 0.6 & rus03 & & & & & 50.0 & & & 50.0 & & & 50.0 \\
\hline 15.64 & -0.24 & 61.8 & ab09 & 0.52 & 4.85 & PMN & & & & kfar & ab09 & 11.6 & 4.1 & 56.8 & 11.0 & 3.9 & 54.8 & 11.7 & 4.2 & 57.1 \\
\hline 16.30 & -0.20 & 49.5 & $\mathrm{pbd}+03$ & 14.00 & 2.70 & $\mathrm{pbd}+03$ & 4.0 & 0.6 & rus 03 & knear & ab09 & 4.1 & 4.7 & 82.1 & & & 82.1 & & & 82.1 \\
\hline 16.40 & -0.50 & 42.7 & $\mathrm{pbd}+03$ & 8.10 & 2.70 & $\mathrm{pbd}+03$ & 4.0 & 0.6 & rus 03 & & & & & 68.4 & & & 68.4 & & & 68.4 \\
\hline 16.40 & -0.20 & 44.5 & $\mathrm{pbd}+03$ & 6.00 & 2.70 & $\mathrm{pbd}+03$ & 4.0 & 0.6 & rus03 & knear & ab09 & 3.9 & 4.9 & 61.9 & & & 61.9 & & & 61.9 \\
\hline 16.58 & -0.05 & 34.7 & swa+04 & 0.28 & 4.86 & swa+04 & & & & kfar & swa+04 & 13.0 & 5.5 & 49.9 & 12.3 & 5.2 & 48.1 & 13.0 & 5.4 & 49.9 \\
\hline 16.60 & -0.30 & 44.9 & $\mathrm{pbd}+03$ & 2.30 & 2.70 & $\mathrm{pbd}+03$ & 4.0 & 0.6 & rus 03 & & & & & 45.0 & & & 45.0 & & & 45.0 \\
\hline 16.90 & -1.10 & & $\mathrm{pbd}+03$ & 1.30 & 2.70 & $\mathrm{pbd}+03$ & 1.6 & 0.25 & rus03 & & & & & 20.2 & & & 20.2 & & & 20.2 \\
\hline 16.90 & 0.80 & 26.3 & $\mathrm{pbd}+03$ & 169.00 & 2.70 & $\mathrm{pbd}+03$ & 2.0 & 25 & rus03 & knear & pdd04 & 2.6 & 6.0 & 118.7 & & & 118.7 & & & 118.7 \\
\hline 17.00 & 0.80 & 24.5 & $\mathrm{pbd}+03$ & 107.00 & 2.70 & $d+03$ & 2.0 & 0.25 & rus03 & knear & pdd04 & 2.5 & 6.2 & 101.9 & & & 101.9 & & & 101.9 \\
\hline 17.00 & 0.90 & 26.4 & $d+03$ & .10 & 2.70 & $\mathrm{pbd}+03$ & 2.0 & 0.25 & rus03 & knear & pdd04 & 2.6 & 6.0 & 59.3 & & & 59.3 & & & 59.3 \\
\hline 17.10 & 0.80 & 27.4 & $\mathrm{pbd}+03$ & 8.80 & 2.70 & $\mathrm{pbd}+03$ & 2.0 & 0.25 & rus 03 & & & & & 44.3 & & & 44.3 & & & 44.3 \\
\hline 17.64 & 0.15 & 25.2 & swa+04 & 0.29 & 4.86 & swa+04 & & & & kfar & swa+04 & 13.7 & 6.2 & 52.3 & 12.9 & 5.8 & 50.2 & 13.6 & 6.1 & 52.0 \\
\hline 18.10 & -0.30 & 53.8 & $\mathrm{pbd}+03$ & 5.80 & 2.70 & $\mathrm{pbd}+03$ & & & & knear & pdd04 & 4.2 & 4.7 & 63.2 & 3.9 & 4.5 & 60.2 & 3.9 & 4.9 & 60.2 \\
\hline 18.20 & .18 & 46.1 & ab09 & 2.60 & 2.70 & $\mathrm{pbd}+03$ & & & & kfar & ab09 & 12.4 & 5.1 & 99.6 & 11.7 & 4.8 & 95.8 & 12.4 & 5.1 & 99.6 \\
\hline 18.20 & 0.40 & 43.3 & $\mathrm{pbd}+03$ & 3.10 & 2.70 & $\mathrm{pbd}+03$ & & & & knear & pdd04 & 3.6 & 5.2 & 46.3 & 3.4 & 4.9 & 44.6 & 3.4 & 5.4 & 44.6 \\
\hline 18.20 & 0.30 & 47.0 & $\mathrm{pbd}+03$ & 27.00 & 2.70 & $\mathrm{pbd}+03$ & & & & knear & pdd04 & 3.8 & 5.0 & 98.8 & 3.6 & 4.8 & 95.3 & 3.6 & 5.2 & 95.3 \\
\hline 18.30 & .40 & 30.5 & $\mathrm{pbd}+03$ & 1.70 & 2.70 & $\mathrm{pbd}+03$ & & & & knear & pdd04 & 2.8 & 5.9 & 32.1 & 2.6 & 5.6 & 30.6 & 2.7 & 6.0 & 31.3 \\
\hline 18.30 & .30 & 50.7 & $\mathrm{pbd}+03$ & 5.40 & 2.70 & $\mathrm{pbd}+03$ & & & & knear & pdd04 & 4.0 & 4.9 & 59.8 & 3.7 & 4.6 & 56.8 & 3.8 & 5.1 & 57.8 \\
\hline 18.30 & 1.90 & 29.9 & $\mathrm{pbd}+03$ & 5.90 & 2.70 & $\mathrm{pbd}+03$ & 2.0 & 0.2 & rus03 & knear & pdd04 & 2.8 & 5.9 & 38.8 & & & 38.8 & & & 38.8 \\
\hline 18.46 & -0.00 & 56.5 & swa+04 & 0.77 & 4.86 & swa+04 & & & & kfar & swa+04 & 11.9 & 4.7 & 65.9 & 11.2 & 4.4 & 63.3 & 11.9 & 4.7 & 65.9 \\
\hline 18.60 & 0.30 & 71.1 & $\mathrm{pbd}+03$ & 2.40 & 2.70 & $\mathrm{pbd}+03$ & 3.4 & 1.0 & rus 03 & & & & & 40.9 & & & 40.9 & & & 40.9 \\
\hline 18.66 & 0.06 & 44.1 & swa+04 & 0.43 & 4.86 & swa+04 & & & & kfar & swa+04 & 12.5 & 5.2 & 56.1 & 11.8 & 4.9 & 54.0 & 12.5 & 5.2 & 56.1 \\
\hline 18.70 & 2.00 & 26.6 & $d+03$ & 88.00 & 2.70 & $\mathrm{pbd}+03$ & 2.0 & 0.2 & rus 03 & knear & pdd04 & 2.5 & 6.2 & 95.5 & & & 95.5 & & & 95.5 \\
\hline 18.90 & 0.50 & 65.5 & $d+03$ & .70 & 2.70 & $d+03$ & 3.4 & 1.0 & $\mathrm{~s} 03$ & knear & pdd04 & 4.6 & 4.4 & 76.6 & & & 76.6 & & & 76.6 \\
\hline 18.90 & .40 & 68.0 & $\mathrm{pbd}+03$ & 18.50 & 2.70 & $\mathrm{pbd}+03$ & 3.4 & 1.0 & rus03 & knear & pdd04 & 4.7 & 4.3 & 80.9 & & & 80.9 & & & 80.9 \\
\hline 18.95 & 0.02 & 52.3 & ab09 & 16 & 4.85 & PMN & 3.4 & 1.0 & rus03 & knear & ab09 & 4.0 & 4.9 & 40.3 & & & 40.3 & & & 40.3 \\
\hline 19.00 & .00 & 48.7 & $b d+03$ & 2.60 & 2.70 & $\mathrm{pbd}+03$ & 3.4 & 1.0 & rus03 & knear & pdd04 & 3.8 & 5.0 & 42.0 & & & 42.0 & & & 42.0 \\
\hline 19.00 & 0.40 & 65.8 & $\mathrm{pbd}+03$ & 7.10 & 2.70 & $\mathrm{pbd}+03$ & 3.4 & 1.0 & rus 03 & knear & ab09 & 4.6 & 4.4 & 58.8 & & & 58.8 & & & 58.8 \\
\hline 19.00 & 0.60 & 68.2 & $\mathrm{pbd}+03$ & 2.00 & 2.70 & $\mathrm{pbd}+03$ & 3.4 & 1.0 & rus 03 & knear & ab09 & 4.7 & 4.3 & 38.5 & & & 38.5 & & & 38.5 \\
\hline 19.07 & -0.28 & 64.2 & $\mathrm{Na}+04$ & 3.40 & 4.86 & swa+04 & 3.4 & 1.0 & rus03 & knear & swa+04 & 4.6 & 4.4 & 46.9 & & & 46.9 & & & 46.9 \\
\hline 19.10 & 0.30 & 66.5 & $\mathrm{pbd}+03$ & 25.00 & 2.70 & $\mathrm{pbd}+03$ & 3.4 & 1.0 & rus03 & knear & pdd04 & 4.7 & 4.4 & 89.4 & & & 89.4 & & & 89.4 \\
\hline 19.12 & 0.34 & 63.5 & $\mathrm{va}+04$ & 1.35 & 4.86 & swa+04 & 3.4 & 1.0 & rus03 & knear & swa+04 & 4.5 & 4.5 & 34.5 & & & 34.5 & & & 34.5 \\
\hline 19.36 & .02 & 51.1 & $a+0$ & 0.33 & 4.86 & swa+04 & & & & kfar & swa+04 & 12.1 & 5.0 & 50.2 & 11.4 & 4.7 & 48.2 & 12.1 & 5.0 & 50.2 \\
\hline 19.50 & 0.10 & 19.9 & $b d+03$ & 3.50 & 2.70 & $\mathrm{pbd}+03$ & 2.0 & 0.2 & rus 03 & & & & & 32.6 & & & 32.6 & & & 32.6 \\
\hline 19.60 & 0.90 & 38.7 & swa+04 & 0.37 & 4.86 & swa+04 & & & & kfar & swa+04 & 12.8 & 5.6 & 54.2 & 12.0 & 5.3 & 51.9 & 12.8 & 5.5 & 54.2 \\
\hline 19.61 & .13 & 58.6 & & 20 & 2.70 & $\mathrm{pbd}+03$ & & & & kfar & ab09 & 11.7 & 4.7 & 112.4 & 11.1 & 4.5 & 108.5 & 11.8 & 4.7 & 113.0 \\
\hline 19.61 & .23 & 41.0 & $\mathrm{kjb}+03$ & .00 & 2.70 & $\mathrm{pbd}+03$ & & & & kfar & $\mathrm{kjb}+03$ & 12.7 & 5.4 & 3.1 & 11.9 & 5.1 & 55.8 & 12.6 & 5.4 & 172.2 \\
\hline .68 & .13 & 5.0 & 3 & & 2.70 & $\mathrm{pbd}+03$ & & & & kfar & $\mathrm{kjb}+03$ & 11.9 & 4.8 & 6.9 & 11.3 & 4.6 & 93.6 & 12.0 & 4.9 & 97.4 \\
\hline .70 & & 3.4 & & & 2.70 & & & & & knear & gg76 & 3.5 & 5.3 & 3.3 & 3.3 & 5. & 70.5 & 3.3 & 5.5 & 70.5 \\
\hline 19.88 & .53 & 50.9 & $\mathrm{Na}+$ & & 4.86 & & & & & kfar & swa +04 & 12.1 & 5.0 & 46.4 & 11.4 & 4. & 44.6 & 12.1 & 5.0 & 46.4 \\
\hline 20.08 & 0.14 & 42.2 & & 60 & 2.70 & $\mathrm{pbd}+03$ & & & & kfar & ab09 & 12.6 & 5.4 & 85.6 & 11.9 & 5.1 & 82.4 & 12.6 & 5.4 & 85.6 \\
\hline 20.50 & 0.20 & 24.1 & $b d+03$ & .40 & 2.70 & $\mathrm{pbd}+03$ & 2.0 & 0.2 & rus 03 & & & & & 28.7 & & & 28.7 & & & 28.7 \\
\hline 20.73 & -0.09 & 56.3 & ab09 & 7.76 & 4.85 & GB6 & & & & kfar & ab09 & 11.8 & 4.9 & 141.5 & 11.2 & 4.6 & 136.7 & 11.9 & 4.9 & 142.3 \\
\hline 21.00 & 0.10 & 18.6 & $b d+03$ & 1.20 & 2.70 & $\mathrm{pbd}+03$ & & & & kfar & pdd04 & 14.2 & 6.9 & 84.3 & 13.3 & 6.5 & 80.7 & 13.9 & 6.7 & 83.1 \\
\hline 21.14 & -0.30 & 38.5 & ab09 & 0.49 & 4.85 & PMN & & & & kfar & ab09 & 12.8 & 5.7 & 59.5 & 12.0 & 5.4 & 57.0 & 12.7 & 5.7 & 59.2 \\
\hline 21.44 & -0.56 & 70.2 & wa+04 & 0.34 & 4.86 & swa+04 & & & & knear & swa+04 & 4.7 & 4.5 & 27.0 & 4.3 & 4.3 & 25.4 & 4.4 & 4.7 & 25.8 \\
\hline 21.67 & -0.26 & 70.1 & ab09 & 0.31 & 4.85 & PMN & & & & knear & ab09 & 4.7 & 4.5 & 26.2 & 4.3 & 4.3 & 24.7 & 4.4 & 4.7 & 25.1 \\
\hline 21.87 & 0.01 & 26.9 & swa+04 & 18 & 4.86 & swa+04 & & & & kfar & swa+04 & 13.5 & 6.4 & 82.6 & 12.7 & 6.0 & 79.3 & 13.3 & 6.3 & 81.8 \\
\hline 21.90 & -0.37 & 79.5 & ab09 & 00 & 2.70 & $\mathrm{pbd}+03$ & & & & kfar & ab09 & 10.7 & 4.3 & 94.7 & 10.2 & 4.1 & 91.7 & 10.9 & 4.4 & 95.9 \\
\hline 22.36 & 0.06 & 82.5 & swa+04 & 50 & 4.86 & swa+04 & & & & knear & swa+04 & 5.1 & 4.2 & 32.4 & 4.8 & 4.0 & 31.1 & 4.8 & 4.5 & 31.1 \\
\hline 22.76 & -0.49 & 74.8 & ab09 & 70 & 2.70 & $\mathrm{pbd}+03$ & & & & knear & ab09 & 4.8 & 4.5 & 53.6 & 4.4 & 4.3 & 0.6 & 4.5 & 4.7 & 51.3 \\
\hline 22.80 & -0.30 & 82.5 & $b d+03$ & 52.30 & 2.70 & $\mathrm{pbd}+03$ & & & & kfar & gg76 & 10.5 & 4.3 & 242.5 & 10.0 & 4.1 & 234.7 & 10.7 & 4.4 & 245.6 \\
\hline 22.90 & -0.30 & 71.1 & $\mathrm{~d}+03$ & & 2.70 & $\mathrm{pbd}+03$ & & & & kfar & pdd04 & 11.0 & 4.6 & 89.6 & 10.4 & 4.4 & 86.3 & 11.1 & 4.7 & 90.1 \\
\hline 22.90 & 0.70 & 99.0 & $\mathrm{~d}+03$ & 0.6 & 2.70 & $\mathrm{pbd}+03$ & & & & knear & pdd04 & 5.8 & 3.9 & 36.8 & 5.3 & 3.7 & 34.7 & 5.4 & 4.1 & 35.1 \\
\hline 23.00 & -0.40 & 74.1 & $\mathrm{pbd}+03$ & 0 & 2.70 & $\mathrm{pbd}+03$ & 4.59 & 0.4 & brm +08 & & & & & 59.0 & & & 59.0 & & & 59.0 \\
\hline & 0.22 & 97.9 & & & 4.85 & PMN & & & & kfar & ab09 & 9.9 & 3.9 & 41.6 & 9.4 & 3.7 & 40.2 & 10.1 & 4.0 & 42.2 \\
\hline 23.10 & 0.60 & 29.5 & $\mathrm{pbd}+03$ & 2.80 & 2.70 & $\mathrm{pbd}+03$ & 1.7 & 0.5 & rus03 & knear & pdd04 & 2.4 & 6.4 & 27.1 & & & 27.1 & & & 27.1 \\
\hline
\end{tabular}


Table A.1. continued.

\begin{tabular}{|c|c|c|c|c|c|c|c|c|c|c|c|c|c|c|c|c|c|c|c|c|}
\hline $\begin{array}{r}l \\
\left({ }^{\circ}\right) \\
(1)\end{array}$ & $\begin{array}{r}b \\
\left({ }^{\circ}\right) \\
(2)\end{array}$ & $\begin{array}{c}V_{\mathrm{lsr}} \\
\mathrm{km} \mathrm{s}^{-1} \\
(3)\end{array}$ & Ref. & $\begin{array}{c}S \\
\text { Jy } \\
(5)\end{array}$ & $\begin{array}{r}\text { Freq } \\
\text { GHz } \\
(6)\end{array}$ & Ref. & $\begin{array}{r}d \\
\mathrm{kpc} \\
(8)\end{array}$ & $\begin{array}{r}d_{\text {erro }} \\
\text { kpc } \\
(9)\end{array}$ & (10) & Mark & Ref. & $\begin{array}{l}D_{8.5} \\
\mathrm{kpc} \\
(13)\end{array}$ & $\begin{array}{l}R_{8.5} \\
\mathrm{kpc} \\
(14)\end{array}$ & $\begin{array}{r}U_{8.5} \\
\mathrm{pc} \mathrm{cm}^{-2} \\
(15)\end{array}$ & $\begin{array}{l}D_{8.0} \\
\mathrm{kpc} \\
(16)\end{array}$ & $\begin{array}{l}R_{8.0} \\
\mathrm{kpc} \\
(17)\end{array}$ & $\begin{array}{r}U_{8.0} \\
\mathrm{pc} \mathrm{cm}^{-2} \\
(18)\end{array}$ & $\begin{array}{l}D_{8.4} \\
\mathrm{kpc} \\
(19)\end{array}$ & $\begin{array}{l}R_{8.4} \\
\mathrm{kpc} \\
(20)\end{array}$ & $\begin{array}{r}U_{8.4} \\
\mathrm{pc} \mathrm{cm}{ }^{-2} \\
(21)\end{array}$ \\
\hline 23.10 & -0.20 & 89.6 & $\mathrm{pbd}+03$ & 2.10 & 2.70 & $\mathrm{pbd}+03$ & & & & kfar & $\begin{array}{ll}1 t 08 \\
\end{array}$ & 10.2 & 4.1 & 81.4 & 9.7 & 3.9 & 78.7 & 10.4 & 4.2 & 82.5 \\
\hline 23.10 & -0.40 & 82.7 & $\mathrm{pbd}+03$ & 3.20 & 2.70 & $\mathrm{pbd}+03$ & & & & knear & lt 08 & 5.1 & 4.3 & 59.0 & 4.7 & 4.1 & 55.9 & 4.8 & 4.5 & 56.7 \\
\hline 23.24 & -0.24 & 76.1 & ab09 & 1.54 & 4.86 & swa+04 & & & & knear & ab09 & 4.8 & 4.5 & 45.3 & 4.5 & 4.3 & 43.4 & 4.5 & 4.7 & 43.4 \\
\hline 23.27 & 0.08 & 78.2 & ab09 & 0.49 & 4.86 & swa+04 & & & & knear & ab09 & 4.9 & 4.4 & 31.4 & 4.6 & 4.2 & 30.1 & 4.6 & 4.6 & 30.1 \\
\hline 23.28 & 0.30 & 108.8 & ab09 & 0.20 & 2.70 & $\mathrm{pbd}+03$ & & & & knear & ab09 & 6.2 & 3.7 & 26.7 & 5.7 & 3.6 & 25.2 & 5.7 & 4.0 & 25.2 \\
\hline 23.42 & -0.21 & 103.0 & lt08 & 19.30 & 2.70 & $\mathrm{pbd}+03$ & 5.88 & 1.2 & brm+08 & kfar & lt08 & 9.6 & 3.8 & 117.1 & & & 117.1 & & & 117.1 \\
\hline 23.46 & -0.20 & 99.0 & ab09 & 7.89 & 4.85 & PMN & & & & knear & ab09 & 5.8 & 3.9 & 88.6 & 5.3 & 3.8 & 83.4 & 5.4 & 4.2 & 84.5 \\
\hline 23.50 & -0.00 & 91.3 & $\mathrm{pbd}+03$ & 9.50 & 2.70 & $\mathrm{pbd}+03$ & & & & kfar & pdd04 & 10.1 & 4.1 & 133.8 & 9.6 & 3.9 & 129.3 & 10.3 & 4.2 & 135.6 \\
\hline 23.61 & -0.38 & 90.1 & ab09 & 1.60 & 2.70 & $\mathrm{pbd}+03$ & & & & knear & ab09 & 5.4 & 4.2 & 48.7 & 5.0 & 4.0 & 46.3 & 5.0 & 4.4 & 46.3 \\
\hline 23.71 & 0.17 & 103.8 & ab09 & 2.60 & 2.70 & $\mathrm{pbd}+03$ & & & & knear & ab09 & 6.0 & 3.8 & 61.4 & 5.5 & 3.7 & 57.9 & 5.5 & 4.1 & 57.9 \\
\hline 23.71 & -0.20 & 76.5 & wa+04 & 0.77 & 4.86 & swa+04 & & & & kfar & swa+04 & 10.7 & 4.5 & 61.4 & 10.2 & 4.3 & 59.5 & 10.9 & 4.6 & 62.2 \\
\hline 23.82 & 0.22 & 107.4 & ab09 & 0.80 & 2.70 & $\mathrm{pbd}+03$ & & & & kfar & ab09 & 9.4 & 3.8 & 55.9 & 9.0 & 3.6 & 54.3 & 9.7 & 3.9 & 57.1 \\
\hline 23.90 & -0.10 & 73.8 & $\mathrm{pbd}+03$ & 1.10 & 2.70 & $\mathrm{pbd}+03$ & & & & kfar & pdd04 & 10.8 & 4.6 & 68.2 & 10.3 & 4.4 & 66.1 & 10.9 & 4.7 & 68.6 \\
\hline 23.96 & 0.15 & 78.9 & ab09 & 2.70 & 2.70 & $\mathrm{pbd}+03$ & 2.5 & 0.4 & cf08 & knear & ab09 & 4.9 & 4.5 & 34.7 & & & 34.7 & & & 34.7 \\
\hline 24.13 & -0.07 & 86.9 & ab09 & 0.40 & 2.70 & $\mathrm{pbd}+03$ & & & & kfar & ab09 & 10.2 & 4.3 & 46.9 & 9.7 & 4.1 & 45.4 & 10.4 & 4.4 & 47.5 \\
\hline 24.14 & 0.12 & 114.5 & ab09 & 0.50 & 2.70 & $\mathrm{pbd}+03$ & & & & kfar & ab09 & 8.9 & 3.7 & 46.1 & 8.6 & 3.5 & 45.1 & 9.4 & 3.8 & 47.8 \\
\hline 24.19 & 0.20 & 111.9 & ab09 & 0.60 & 2.70 & $\mathrm{pbd}+03$ & & & & kfar & ab09 & 9.1 & 3.7 & 49.7 & 8.7 & 3.6 & 48.2 & 9.5 & 3.9 & 51.1 \\
\hline 24.20 & -0.10 & 83.6 & $\mathrm{pbd}+03$ & 0.80 & 2.70 & $\mathrm{pbd}+03$ & & & & kfar & pdd04 & 10.4 & 4.4 & 59.8 & 9.8 & 4.2 & 57.5 & 10.5 & 4.5 & 60.2 \\
\hline 24.39 & 0.07 & 110.0 & ab09 & 2.80 & 2.70 & $\mathrm{pbd}+03$ & & & & kfar & ab09 & 9.1 & 3.8 & 83.1 & 8.8 & 3.6 & 81.3 & 9.5 & 3.9 & 85.5 \\
\hline 24.47 & 0.49 & 99.8 & ab09 & 13.00 & 2.70 & $\mathrm{pbd}+03$ & & & & knear & ab09 & 5.8 & 4.0 & 102.6 & 5.4 & 3.8 & 97.8 & 5.4 & 4.2 & 97.8 \\
\hline 24.48 & 0.21 & 115.7 & ab09 & 18.00 & 2.70 & $\mathrm{pbd}+03$ & & & & kfar & ab09 & 8.8 & 3.7 & 151.1 & 8.5 & 3.5 & 147.6 & 9.3 & 3.8 & 156.8 \\
\hline 24.50 & -0.04 & 108.1 & ab09 & 0.90 & 4.86 & swa+04 & & & & kfar & ab09 & 9.2 & 3.8 & 58.5 & 8.8 & 3.7 & 56.8 & 9.6 & 4.0 & 60.2 \\
\hline 24.51 & -0.22 & 44.3 & ab09 & 4.19 & 4.85 & PMN & & & & knear & ab09 & 3.2 & 5.7 & 48.3 & 3.0 & 5.4 & 46.3 & 3.1 & 5.8 & 47.3 \\
\hline 24.51 & -0.22 & 96.4 & ab09 & 19.00 & 2.70 & $\mathrm{pbd}+03$ & & & & kfar & ab09 & 9.8 & 4.1 & 165.2 & 9.3 & 3.9 & 159.5 & 10.0 & 4.2 & 167.4 \\
\hline 24.68 & -0.16 & 44.0 & ab09 & 4.91 & 4.85 & PMN & & & & knear & ab09 & 3.2 & 5.7 & 50.9 & 3.0 & 5.4 & 48.8 & 3.1 & 5.9 & 49.8 \\
\hline 24.68 & -0.16 & 111.1 & ab09 & 9.90 & 2.70 & $\mathrm{pbd}+03$ & & & & kfar & ab09 & 9.0 & 3.8 & 125.6 & 8.7 & 3.6 & 122.8 & 9.4 & 3.9 & 129.3 \\
\hline 24.70 & -0.10 & 101.1 & $b d+03$ & 23.40 & 2.70 & $\mathrm{pbd}+03$ & & & & knear & pdd04 & 5.9 & 4.0 & 126.3 & 5.4 & 3.8 & 119.1 & 5.4 & 4.2 & 119.1 \\
\hline 24.70 & -0.20 & 84.8 & $\mathrm{pbd}+03$ & 3.20 & 2.70 & $d+03$ & 2.2 & 0.25 & rus03 & & & & & 33.7 & & & 33.7 & & & 33.7 \\
\hline 24.81 & 0.10 & 108.6 & ab09 & 13.30 & 2.70 & $\mathrm{pbd}+03$ & & & & near & ab09 & 6.3 & 3.8 & 109.3 & 5.8 & 3.7 & 103.4 & 5.7 & 4.1 & 102.2 \\
\hline 25.16 & 0.06 & 47.0 & ab09 & 0.80 & 2.70 & $\mathrm{pbd}+03$ & & & & knear & ab09 & 3.4 & 5.7 & 28.4 & 3.1 & 5.3 & 26.7 & 3.2 & 5.8 & 27.3 \\
\hline 25.26 & -0.32 & 63.7 & ab09 & 2.00 & 2.70 & $\mathrm{pbd}+03$ & & & & kfar & ab09 & 11.2 & 5.0 & 85.3 & 10.6 & 4.8 & 82.2 & 11.2 & 5.1 & 85.3 \\
\hline 25.30 & 0.30 & 40.3 & $\mathrm{pbd}+03$ & 2.30 & 2.70 & $\mathrm{pbd}+03$ & 2.2 & 0.25 & rus03 & & & & & 30.2 & & & 30.2 & & & 30.2 \\
\hline 25.40 & -0.14 & 98.4 & ab09 & 16.19 & 4.85 & PMN & & & & knear & ab09 & 5.8 & 4.1 & 112.6 & 5.3 & 3.9 & 106.0 & 5.3 & 4.3 & 106.0 \\
\hline 25.40 & -0.30 & 62.5 & $b d+03$ & 1.60 & 2.70 & $\mathrm{pbd}+03$ & & & & kfar & pdd04 & 11.2 & 5.1 & 79.2 & 10.6 & 4.8 & 76.3 & 11.3 & 5.1 & 79.7 \\
\hline 25.40 & -0.20 & 59.1 & $d+03$ & 26.00 & 2.70 & $\mathrm{pbd}+03$ & & & & kfar & pdd04 & 11.4 & 5.2 & 202.9 & 10.8 & 4.9 & 195.7 & 11.4 & 5.2 & 202.9 \\
\hline 25.40 & 0.00 & -13.7 & $d+03$ & 1.40 & 2.70 & $\mathrm{pbd}+03$ & & & & kout & hh09 & 17.2 & 10.2 & 100.8 & 15.9 & 9.4 & 95.7 & 16.4 & 9.4 & 97.6 \\
\hline 25.70 & 0.00 & 56.3 & $\mathrm{pbd}+03$ & 2.60 & 2.70 & $\mathrm{pbd}+03$ & & & & kfar & pdd04 & 11.5 & 5.3 & 94.7 & 10.9 & 5.0 & 91.4 & 11.5 & 5.3 & 94.7 \\
\hline 25.77 & 0.21 & 110.8 & ab09 & 2.28 & 4.86 & swa+04 & & & & knear & ab09 & 6.5 & 3.9 & 63.2 & 5.9 & 3.7 & 59.2 & 5.9 & 4.1 & 59.2 \\
\hline 25.79 & -0.14 & 19.5 & $\mathrm{Na}+04$ & 0.42 & 4.86 & swa+04 & & & & ktan & swa+04 & 7.7 & 3.7 & 40.3 & & & & & & \\
\hline 25.80 & 0.24 & 9.5 & ab09 & 5.01 & 4.85 & PMN & & & & knear & ab09 & 7.3 & 3.7 & 88.8 & 6.4 & 3.6 & 81.3 & 6.3 & 3.9 & 80.5 \\
\hline 26.10 & -0.10 & 76.9 & $\mathrm{pbd}+03$ & 8.90 & 2.70 & $\mathrm{pbd}+03$ & & & & kfar & pdd04 & 10.5 & 4.7 & 134.4 & 9.9 & 4.5 & 129.2 & 10.6 & 4.8 & 135.3 \\
\hline 26.40 & 1.80 & 43.0 & & 0.20 & 2.70 & $\mathrm{pbd}+03$ & 2.3 & 0.7 & rus03 & & & & & 13.8 & & & 13.8 & & & 13.8 \\
\hline 26.54 & 0.41 & 88.4 & +03 & 3.20 & 2.70 & $\mathrm{pbd}+03$ & & & & kfar & +03 & 9.9 & 4.4 & 91.9 & 9.4 & 4.2 & 88.8 & 10.1 & 4.5 & 93.1 \\
\hline 26.56 & -0.31 & 04.2 & ab09 & 2.30 & 2.70 & $\mathrm{pbd}+03$ & & & & kfar & ab09 & 9.1 & 4.1 & 77.8 & 8.7 & 3.9 & 75.5 & 9.4 & 4.2 & 79.5 \\
\hline 26.60 & 0.09 & 102.5 & ab09 & 1.18 & 4.85 & PMN & & & & kfar & ab09 & 9.1 & 4.1 & 63.5 & 8.8 & 3.9 & 62.1 & 9.5 & 4.2 & 65.3 \\
\hline 26.60 & -0.11 & 69.2 & ab09 & 5.70 & 2.70 & $\mathrm{pbd}+03$ & & & & kfar & ab09 & 10.8 & 5.0 & 118.0 & 10.2 & 4.7 & 113.6 & 10.9 & 5.0 & 118.7 \\
\hline 26.98 & -0.07 & 79.9 & ab09 & 1.10 & 2.70 & $\mathrm{pbd}+03$ & & & & kfar & ab09 & 10.2 & 4.7 & 65.7 & 9.7 & 4.4 & 63.5 & 10.4 & 4.8 & 66.6 \\
\hline 27.30 & 0.10 & 36.3 & $b d+03$ & 1.40 & 2.70 & $\mathrm{pbd}+03$ & & & & kfar & pdd04 & 12.5 & 6.3 & 81.5 & 11.7 & 5.9 & 78.0 & 12.4 & 6.2 & 81.1 \\
\hline 27.31 & -0.14 & 92.3 & ab09 & 8.50 & 2.70 & $\mathrm{pbd}+03$ & & & & knear & ab09 & 5.5 & 4.4 & 86.0 & 5.1 & 4.2 & 81.8 & 5.1 & 4.6 & 81.8 \\
\hline 27.50 & 0.20 & 33.9 & $d+03$ & 3.00 & 2.70 & $\mathrm{pbd}+03$ & & & & kfar & pdd04 & 12.6 & 6.4 & 105.6 & 11.8 & 6.0 & 101.1 & 12.5 & 6.3 & 105.0 \\
\hline 27.98 & 0.08 & 76.6 & swa+04 & 0.33 & 4.86 & swa+04 & & & & kfar & swa+04 & 10.3 & 4.8 & 45.1 & 9.7 & 4.6 & 43.3 & 10.4 & 4.9 & 45.4 \\
\hline 28.00 & 0.32 & 92.7 & ab09 & 0.39 & 4.85 & PMN & & & & kfar & ab09 & 9.4 & 4.4 & 44.9 & 9.0 & 4.2 & 43.6 & 9.7 & 4.6 & 45.9 \\
\hline 28.00 & -0.03 & 99.9 & ab09 & 1.00 & 2.70 & $\mathrm{pbd}+03$ & & & & kfar & ab09 & 9.0 & 4.3 & 58.5 & 8.6 & 4.1 & 56.8 & 9.4 & 4.4 & 60.2 \\
\hline 28.15 & 0.15 & 89.2 & ab09 & 0.26 & 4.85 & PMN & & & & knear & ab09 & 5.4 & 4.5 & 27.1 & 5.0 & 4.3 & 25.7 & 5.0 & 4.7 & 25.7 \\
\hline 28.25 & 0.01 & 110.3 & ab09 & 0.33 & 4.85 & PMN & & & & kfar & ab09 & 8.1 & 4.1 & 38.4 & 7.9 & 3.9 & 37.8 & 8.8 & 4.2 & 40.6 \\
\hline 28.30 & -0.38 & 44.8 & pmg08 & 0.37 & 1.40 & $\operatorname{ccg}+98$ & & & & kfar & $\mathrm{mg} 08$ & 11.9 & 5.9 & 49.5 & 11.2 & 5.6 & 47.5 & 11.8 & 5.9 & 49.2 \\
\hline 28.30 & -0.38 & 75.6 & pmg08 & 0.70 & 1.40 & $\operatorname{ccg}+98$ & & & & kfar & pmg08 & 10.3 & 4.9 & 55.6 & 9.7 & 4.6 & 53.4 & 10.4 & 5.0 & 56.0 \\
\hline 28.44 & 0.00 & 96.9 & ab09 & 0.90 & 2.70 & $\mathrm{pbd}+03$ & & & & knear & ab09 & 5.8 & 4.4 & 42.1 & 5.4 & 4.2 & 40.1 & 5.3 & 4.6 & 39.6 \\
\hline 28.60 & 0.00 & 96.7 & $d+03$ & 14.30 & 2.70 & $\mathrm{pbd}+03$ & & & & kfar & pdd04 & 9.1 & 4.4 & 143.1 & 8.7 & 4.2 & 138.9 & 9.4 & 4.5 & 146.2 \\
\hline 28.60 & -0.36 & 89.0 & swa+04 & 0.78 & 4.86 & swa+04 & & & & knear & swa+04 & 5.4 & 4.6 & 39.1 & 5.0 & 4.3 & 37.1 & 5.0 & 4.8 & 37.1 \\
\hline 28.61 & 0.02 & 1( & ab09 & & 4.85 & & & & & kfar & ab09 & 8.8 & 4.3 & 94.7 & 8.4 & 4.1 & 91.8 & 9.2 & 4.4 & 97.5 \\
\hline & 0.19 & 103.8 & ab09 & 80 & 2.70 & $\mathrm{pbd}+03$ & & & & ktan & ab09 & 7.5 & 4.1 & 48.1 & & & & & & \\
\hline & 0.00 & 102.3 & $\mathrm{pbd}+03$ & 6.80 & 2.70 & $\mathrm{pbd}+03$ & & & & kfar & pdd04 & 8.7 & 4.3 & 108.4 & 8.4 & 4.1 & 105.9 & 9.1 & 4.4 & 111.7 \\
\hline & 25 & 98.8 & $\mathrm{ab0}$ & & 2.70 & & & & & $\mathrm{kf}$ & ab09 & 8.9 & 4.3 & 65.0 & 8.5 & 4.1 & 63.0 & 9.3 & 4.5 & 66.9 \\
\hline 28.80 & 0.17 & 107.6 & ab09 & 6.00 & 2.70 & $\mathrm{pbd}+03$ & & & & kfar & ab09 & 8.2 & 4.2 & 99.9 & 8.0 & 4.0 & 98.3 & 8.8 & 4.3 & 104.7 \\
\hline
\end{tabular}


Table A.1. continued.

\begin{tabular}{|c|c|c|c|c|c|c|c|c|c|c|c|c|c|c|c|c|c|c|c|c|}
\hline $\begin{array}{r}l \\
\left({ }^{\circ}\right) \\
(1) \\
\end{array}$ & $\begin{array}{r}b \\
\left({ }^{\circ}\right) \\
(2) \\
\end{array}$ & $\begin{array}{c}V_{\mathrm{lsr}} \\
\mathrm{km} \mathrm{s}^{-1} \\
(3)\end{array}$ & Ref. & $\begin{array}{c}S \\
\text { Jy } \\
(5)\end{array}$ & $\begin{array}{r}\text { Freq } \\
\text { GHz } \\
(6)\end{array}$ & Ref. & $\begin{array}{r}d \\
\mathrm{kpc} \\
(8)\end{array}$ & $\begin{array}{r}d_{\text {erro }} \\
\text { kpc } \\
(9)\end{array}$ & (10) & Mark & Ref. & $\begin{array}{r}D_{8.5} \\
\mathrm{kpc} \\
(13)\end{array}$ & $\begin{array}{l}R_{8.5} \\
\mathrm{kpc} \\
(14)\end{array}$ & $\begin{array}{r}U_{8.5} \\
\mathrm{pc} \mathrm{cm}^{-2} \\
(15) \\
\end{array}$ & $\begin{array}{r}D_{8.0} \\
\text { kpc } \\
(16)\end{array}$ & $\begin{array}{l}R_{8.0} \\
\mathrm{kpc} \\
(17)\end{array}$ & $\begin{array}{r}U_{8.0} \\
\mathrm{pc} \mathrm{cm}^{-2} \\
(18)\end{array}$ & $\begin{array}{c}D_{8.4} \\
\mathrm{kpc} \\
(19)\end{array}$ & $\begin{array}{l}R_{8.4} \\
\mathrm{kpc} \\
(20)\end{array}$ & $\begin{array}{r}U_{8.4} \\
\mathrm{pc} \mathrm{cm}^{-2} \\
(21)\end{array}$ \\
\hline 28.80 & 3.50 & 0.6 & $\mathrm{pbd}+03$ & 34.00 & 2.70 & $\mathrm{pbd}+03$ & & & & kout & hh09 & 15.0 & 8.6 & 266.5 & & & & 14.5 & 8.2 & 260.5 \\
\hline 28.82 & -0.23 & 90.6 & ab09 & 1.40 & 2.70 & $\mathrm{pbd}+03$ & & & & knear & ab09 & 5.5 & 4.5 & 47.1 & 5.1 & 4.3 & 44.8 & 5.1 & 4.7 & 44.8 \\
\hline 28.86 & 0.06 & 101.7 & ab09 & 0.60 & 4.86 & swa+04 & & & & kfar & ab09 & 8.7 & 4.3 & 49.2 & 8.4 & 4.1 & 48.1 & 9.1 & 4.4 & 50.7 \\
\hline 28.90 & 3.50 & 1.1 & $\mathrm{pbd}+03$ & 33.40 & 2.70 & $\mathrm{pbd}+03$ & & & & kout & hh09 & 14.9 & 8.5 & 263.7 & & & & 14.5 & 8.1 & 259.0 \\
\hline 29.00 & -0.60 & 52.6 & $\mathrm{pbd}+03$ & 4.00 & 2.70 & $\mathrm{pbd}+03$ & 3.3 & 0.7 & rus03 & & & & & 47.6 & & & 47.6 & & & 47.6 \\
\hline 29.10 & -0.70 & 47.3 & $d+03$ & 1.20 & 2.70 & $\mathrm{pbd}+03$ & 3.3 & 0.7 & rus03 & knear & pdd04 & 3.2 & 5.9 & 31.9 & & & 31.9 & & & 31.9 \\
\hline 29.10 & -0.00 & 52.4 & $d+03$ & 2.10 & 2.70 & $\mathrm{pbd}+03$ & 3.3 & 0.7 & rus03 & & & & & 38.4 & & & 38.4 & & & 38.4 \\
\hline 29.14 & 0.43 & 21.9 & ab09 & 2.70 & 2.70 & $\mathrm{pbd}+03$ & & & & kfar & ab09 & 13.2 & 7.1 & 105.2 & 12.4 & 6.6 & 100.9 & 13.0 & 6.9 & 104.1 \\
\hline 29.20 & 0.00 & 61.7 & $\mathrm{pbd}+03$ & 6.60 & 2.70 & $\mathrm{pbd}+03$ & 3.3 & 0.7 & rus03 & & & & & 56.2 & & & 56.2 & & & 56.2 \\
\hline 29.90 & -0.00 & 97.5 & $\mathrm{pbd}+03$ & 19.00 & 2.70 & $\mathrm{pbd}+03$ & & & & kfar & pdd04 & 8.7 & 4.5 & 152.6 & 8.4 & 4.2 & 149.1 & 9.1 & 4.6 & 157.2 \\
\hline 29.96 & -0.02 & 91.3 & swa+04 & 6.02 & 4.86 & swa+04 & & & & kfar & swa +04 & 9.1 & 4.6 & 109.3 & 8.7 & 4.4 & 106.1 & 9.4 & 4.7 & 111.7 \\
\hline 30.06 & -0.33 & 101.3 & ab09 & 0.11 & 4.85 & PMN & & & & knear & ab09 & 6.3 & 4.4 & 22.5 & 5.8 & 4.2 & 21.3 & 5.7 & 4.6 & 21.0 \\
\hline 30.07 & -0.16 & 98.2 & ab09 & 1.00 & 2.70 & $\mathrm{pbd}+03$ & & & & kfar & ab09 & 8.6 & 4.4 & 56.8 & 8.3 & 4.2 & 55.5 & 9.1 & 4.6 & 59.0 \\
\hline 30.23 & -0.15 & 99.4 & ab09 & 3.50 & 2.70 & $\mathrm{pbd}+03$ & & & & knear & ab09 & 6.2 & 4.4 & 69.3 & 5.7 & 4.2 & 65.5 & 5.6 & 4.6 & 64.8 \\
\hline 30.28 & -0.02 & 97.9 & ab09 & 1.50 & 2.70 & $\mathrm{pbd}+03$ & & & & knear & ab09 & 6.1 & 4.5 & 51.7 & 5.6 & 4.3 & 48.8 & 5.5 & 4.7 & 48.3 \\
\hline 30.38 & -0.11 & 99.8 & ab09 & 0.72 & 4.86 & swa +04 & & & & knear & ab09 & 6.3 & 4.4 & 42.2 & 5.7 & 4.2 & 39.5 & 5.6 & 4.6 & 39.0 \\
\hline 30.40 & -0.24 & 102.5 & ab09 & 8.50 & 2.70 & $\mathrm{pbd}+03$ & & & & kfar & ab09 & 8.1 & 4.4 & 111.3 & 7.9 & 4.2 & 109.5 & 8.7 & 4.5 & 116.7 \\
\hline 30.42 & 0.46 & 22.5 & swa+04 & 0.31 & 4.86 & swa +04 & & & & kfar & swa+04 & 13.0 & 7.1 & 51.6 & 12.2 & 6.7 & 49.5 & 12.8 & 6.9 & 51.1 \\
\hline 30.50 & -0.29 & 107.3 & ab09 & 3.20 & 2.70 & $d+03$ & & & & kfar & ab09 & 7.8 & 4.3 & 78.4 & 7.3 & 4.1 & 75.0 & 8.4 & 4.4 & 82.4 \\
\hline 30.50 & 0.00 & 49.6 & $\mathrm{pbd}+03$ & 1.50 & 2.70 & $\mathrm{pbd}+03$ & & & & knear & pdd04 & 3.3 & 5.9 & 34.3 & 3.1 & 5.5 & 32.9 & 3.2 & 6.0 & 33.6 \\
\hline 30.50 & 0.40 & 57.7 & $\mathrm{pbd}+03$ & 2.20 & 2.70 & $\mathrm{pbd}+03$ & & & & knear & pdd04 & 3.8 & 5.6 & 42.8 & 3.5 & 5.3 & 40.5 & 3.6 & 5.7 & 41.3 \\
\hline 30.54 & 0.02 & 46.1 & ab09 & 1.61 & 4.85 & PMN & & & & kfar & ab09 & 11.5 & 6.0 & 82.3 & 10.8 & 5.7 & 78.9 & 11.5 & 6.0 & 82.3 \\
\hline 30.54 & -0.45 & 106.5 & ab09 & 2.00 & 2.70 & $\mathrm{pbd}+03$ & & & & kfar & ab09 & 7.7 & 4.3 & 66.4 & 7.4 & 4.1 & 64.7 & 8.4 & 4.5 & 70.4 \\
\hline 30.60 & -0.10 & 102.5 & $\mathrm{pbd}+03$ & 2.60 & 2.70 & $\mathrm{pbd}+03$ & & & & ktan & pdd04 & 7.3 & 4.3 & 70.0 & & & & & & \\
\hline 30.60 & -0.60 & 17.8 & rus03 & & & & 0.5 & 0.1 & rus 03 & & & & & & & & & & & \\
\hline 30.69 & -0.26 & 98.5 & ab09 & 3.20 & 2.70 & $\mathrm{pbd}+03$ & & & & kfar & ab09 & 8.4 & 4.5 & 82.3 & 8.1 & 4.3 & 80.3 & 8.9 & 4.6 & 85.5 \\
\hline 30.80 & -0.00 & 91.8 & $\mathrm{pbd}+03$ & 95.00 & 2.70 & $\mathrm{pbd}+03$ & & & & knear & pmg08 & 8.9 & 4.6 & 265.0 & 5.2 & 4.4 & 185.2 & 5.2 & 4.8 & 185.2 \\
\hline 30.85 & 0.13 & 100.0 & kb94 & 1.80 & 2.70 & $d+03$ & & & & ktan & kb94 & 7.3 & 4.4 & 61.9 & & & & & & \\
\hline 31.00 & 0.00 & 101.2 & $\mathrm{pbd}+03$ & 37.00 & 2.70 & $\mathrm{pbd}+03$ & & & & kfar & pdd04 & 8.0 & 4.4 & 180.2 & 7.8 & 4.2 & 177.2 & 8.6 & 4.6 & 189.1 \\
\hline 31.05 & 0.48 & 27.9 & ab09 & 1.70 & 2.70 & $\mathrm{pbd}+03$ & & & & kfar & ab09 & 12.6 & 6.9 & 87.4 & 11.8 & 6.4 & 83.7 & 12.4 & 6.7 & 86.5 \\
\hline 31.13 & 0.28 & 104.7 & ab09 & 1.20 & 2.70 & $d+03$ & & & & kfar & ab09 & 7.7 & 4.4 & 56.0 & 7.4 & 4.2 & 54.5 & 8.4 & 4.5 & 59.3 \\
\hline 31.17 & -0.13 & 41.4 & kb94 & 1.60 & 2.70 & +03 & & & & kfar & kb94 & 11.7 & 6.2 & 81.5 & 11.0 & 5.9 & 78.2 & 11.6 & 6.2 & 81.0 \\
\hline 31.24 & -0.11 & 98.0 & $\mathrm{kjb}+03$ & 3.40 & 2.70 & $d+03$ & & & & ktan & $\mathrm{kjb}+03$ & 7.3 & 4.4 & 76.5 & & & & & & \\
\hline 31.28 & 0.06 & 104.7 & ab09 & 0.70 & 2.70 & $\mathrm{pbd}+03$ & & & & knear & ab09 & 6.8 & 4.4 & 43.1 & 6.4 & 4.2 & 41.4 & 6.0 & 4.6 & 39.6 \\
\hline 31.40 & -0.26 & 86.2 & ab09 & 6.00 & 2.70 & $\mathrm{pbd}+03$ & & & & kfar & ab09 & 9.1 & 4.8 & 107.1 & 8.7 & 4.6 & 103.9 & 9.4 & 4.9 & 109.4 \\
\hline 31.41 & 0.31 & 100.9 & ab09 & 1.43 & 4.86 & swa +04 & & & & knear & ab09 & 6.6 & 4.5 & 54.7 & 6.0 & 4.3 & 51.3 & 5.8 & 4.7 & 50.2 \\
\hline 31.60 & 0.10 & 99.9 & $\mathrm{pbd}+03$ & 0.90 & 2.70 & $\mathrm{pbd}+03$ & & & & knear & pdd04 & 6.5 & 4.5 & 45.5 & 5.9 & 4.3 & 42.7 & 5.7 & 4.7 & 41.7 \\
\hline 31.90 & 1.40 & 53.0 & rus 03 & 1.80 & 2.70 & $\mathrm{pbd}+03$ & 3.8 & 1.1 & rus03 & knear & pdd04 & 3.6 & 5.8 & 40.1 & & & 40.1 & & & 40.1 \\
\hline 32.20 & 0.10 & 96.7 & $\mathrm{pbd}+03$ & 0.90 & 2.70 & $\mathrm{pbd}+03$ & & & & kfar & pdd04 & 8.1 & 4.6 & 52.7 & 7.8 & 4.4 & 51.4 & 8.6 & 4.7 & 54.8 \\
\hline 32.80 & 0.20 & 15.5 & $\mathrm{pbd}+03$ & 1.70 & 2.70 & $\mathrm{pbd}+03$ & & & & kfar & pdd04 & 13.2 & 7.6 & 90.1 & 12.3 & 7.1 & 86.0 & 12.9 & 7.3 & 88.7 \\
\hline 32.97 & 0.04 & 93.2 & ng08 & 0.16 & 1.40 & $\operatorname{ccg}+98$ & & & & kfar & pmg08 & 8.2 & 4.7 & 29.2 & 7.9 & 4.5 & 28.5 & 8.6 & 4.9 & 30.1 \\
\hline 33.13 & -0.09 & 87.4 & ab09 & 0.50 & 4.86 & ahck02 & & & & knear & ab09 & 5.6 & 4.9 & 34.5 & 5.2 & 4.6 & 32.8 & 5.1 & 5.1 & 32.4 \\
\hline 33.20 & -0.01 & 105.8 & ab09 & 0.29 & 4.86 & was +03 & & & & kfar & ab09 & 8.4 & 4.5 & 37.7 & 7.7 & 4.3 & 35.6 & 7.0 & 4.7 & 33.4 \\
\hline 33.24 & 0.01 & 35.1 & was +03 & 0.46 & 4.86 & was +03 & & & & kfar & was +03 & 11.8 & 6.6 & 55.2 & 11.1 & 6.2 & 53.0 & 11.6 & 6.5 & 54.6 \\
\hline 33.40 & -0.00 & 76.5 & $d+03$ & 0.80 & 2.70 & $\mathrm{pbd}+03$ & & & & kfar & pdd04 & 9.3 & 5.2 & 55.5 & 8.8 & 4.9 & 3.5 & 9.5 & 5.3 & 56.3 \\
\hline 33.81 & -0.19 & 40.3 & was +03 & 0.15 & 4.86 & was +03 & & & & kfar & was +03 & 11.4 & 6.4 & 37.1 & 10.7 & 6.0 & 35.6 & 11.3 & 6.3 & 36.9 \\
\hline 33.92 & 0.11 & 99.0 & ab09 & 0.83 & 4.86 & & & & & $\mathrm{kf}$ & ab09 & 7.9 & 4.7 & 51.4 & 7.2 & 4.4 & 8.3 & 7.8 & 4.8 & 51.0 \\
\hline 34.09 & 0.44 & 32.6 & ab09 & 0.18 & 4.86 & was- & & & & kfar & ab09 & 11.8 & 6.8 & 40.4 & 11.1 & 6.3 & 38.8 & 11.7 & 6.6 & 40.2 \\
\hline 34.26 & 0.15 & 54.0 & hck02 & & 4.86 & & & & & knear & ahck02 & 3.5 & 5.9 & 56.4 & 3.3 & 5.6 & 54.2 & 3.4 & 6.0 & 55.3 \\
\hline 34.30 & 0.10 & 54.5 & $\mathrm{pbd}+03$ & 15.00 & 2.70 & $\mathrm{pbd}+03$ & & & & knear & pdd04 & 3.6 & 5. & 78.3 & 3.4 & 5.6 & 75.4 & 3.4 & 6.0 & 75.4 \\
\hline 34.40 & 0.23 & 60.1 & ab09 & 0.06 & 4.86 & was +03 & & & & kfar & ab09 & 10.1 & 5.7 & 25.2 & 9.5 & 5.4 & 24.2 & 10.2 & 5.7 & 25.4 \\
\hline 34.76 & -0.68 & 52.1 & ab09 & 2.80 & 2.70 & $\mathrm{pbd}+03$ & & & & knear & ab09 & 3.4 & 6.0 & 43.1 & 3.2 & 5.7 & 41.4 & 3.3 & 6.1 & 42.3 \\
\hline 34.93 & -0.02 & 45.6 & ab09 & 1.90 & 2.70 & $\mathrm{pbd}+03$ & & & & kfar & ab09 & 10.9 & 6.3 & 82.3 & 10.2 & 5.9 & 78.7 & 10.8 & 6.2 & 81.8 \\
\hline 35.02 & 0.35 & 57.2 & ab09 & 0.04 & 4.86 & was +03 & & & & knear & ab09 & 3.7 & 5.8 & 11.3 & 3.5 & 5.5 & 10.9 & 3.6 & 5.9 & 11.1 \\
\hline 35.04 & -0.50 & 48.0 & as +03 & 0.31 & 4.86 & was +03 & & & & kfar & was +03 & 10.7 & 6.2 & 45.3 & 10.1 & 5.8 & 43.6 & 10.7 & 6.1 & 45.3 \\
\hline 35.10 & -1.50 & 44.2 & $d+03$ & 3.00 & 2.70 & $\mathrm{pbd}+03$ & 3.27 & 0.5 & $\mathrm{zzr}+08$ & knear & pdd04 & 3.0 & 6.3 & 42.4 & & & 42.4 & & & 42.4 \\
\hline 35.20 & -1.70 & 46.5 & $\mathrm{pbd}+03$ & 14.80 & 2.70 & $\mathrm{pbd}+03$ & 3.27 & 0.5 & $\mathrm{zzr}+08$ & & & & & 72.1 & & & 72.1 & & & 72.1 \\
\hline 35.20 & -1.80 & 47.1 & $\mathrm{pbd}+03$ & 19.00 & 2.70 & $\mathrm{pbd}+03$ & & & & knear & pdd04 & 3.1 & 6.2 & 76.7 & 3.0 & 5.8 & 75.0 & 3.0 & 6.3 & 75.0 \\
\hline 35.30 & -1.80 & 46.6 & $\mathrm{pbd}+03$ & 5.10 & 2.70 & $\mathrm{pbd}+03$ & & & & knear & pdd04 & 3.1 & 6.2 & 49.5 & 2.9 & 5.9 & 47.3 & 3.0 & 6.3 & 48.4 \\
\hline 35.57 & -0.03 & 51.8 & ahck02 & 0.47 & 4.86 & ahck02 & & & & knear & ahck02 & 3.4 & 6.1 & 24.2 & 3.2 & 5.7 & 23.2 & 3.3 & 6.1 & 23.7 \\
\hline 35.58 & 0.07 & 47.6 & ahck02 & 0.53 & 4.86 & ahck02 & & & & knear & ahck02 & 3.2 & 6.2 & 24.2 & 3.0 & 5.8 & 23.2 & 3.1 & 6.3 & 23.7 \\
\hline 35.60 & -0.50 & 56.0 & $\mathrm{pbd}+03$ & 4.30 & 2.70 & $\mathrm{pbd}+03$ & & & & knear & pdd04 & 3.7 & 5.9 & 52.6 & 3.5 & 5.6 & 50.7 & 3.5 & 6.0 & 50.7 \\
\hline 35.60 & 0.10 & 50.5 & $\mathrm{pbd}+03$ & 1.70 & 2.70 & $\mathrm{pbd}+03$ & & & & kfar & pdd04 & 10.5 & 6.1 & 77.4 & 9.9 & 5.7 & 74.4 & 10.4 & 6.1 & 76.9 \\
\hline
\end{tabular}


Table A.1. continued.

\begin{tabular}{|c|c|c|c|c|c|c|c|c|c|c|c|c|c|c|c|c|c|c|c|c|}
\hline $\begin{array}{r}l \\
\left({ }^{\circ}\right) \\
(1)\end{array}$ & $\begin{array}{r}b \\
\left({ }^{\circ}\right) \\
(2)\end{array}$ & $\begin{array}{c}V_{\mathrm{lsr}} \\
\mathrm{km} \mathrm{s}^{-1} \\
(3)\end{array}$ & Ref. & $\begin{array}{c}S \\
\text { Jy } \\
(5)\end{array}$ & $\begin{array}{r}\text { Freq } \\
\mathrm{GHz} \\
(6)\end{array}$ & Ref. & $\begin{array}{r}d \\
\mathrm{kpc} \\
(8)\end{array}$ & $\begin{array}{r}d_{\text {erro }} \\
\mathrm{kpc} \\
(9)\end{array}$ & Ref. & Mark & Ref. & $\begin{array}{r}D_{8.5} \\
\mathrm{kpc} \\
(13)\end{array}$ & $\begin{array}{r}R_{8.5} \\
\mathrm{kpc} \\
(14)\end{array}$ & $\begin{array}{r}U_{8.5} \\
\mathrm{pc} \mathrm{cm}^{-2} \\
(15)\end{array}$ & $\begin{array}{r}D_{8.0} \\
\mathrm{kpc} \\
(16)\end{array}$ & $\begin{array}{l}R_{8.0} \\
\mathrm{kpc} \\
(17)\end{array}$ & $\begin{array}{r}U_{8.0} \\
\mathrm{pc} \mathrm{cm}^{-2} \\
(18)\end{array}$ & $\begin{array}{r}D_{8.4} \\
\mathrm{kpc} \\
(19)\end{array}$ & $\begin{array}{l}R_{8.4} \\
\mathrm{kpc} \\
(20)\end{array}$ & $\begin{array}{r}U_{8.4} \\
\mathrm{pc} \mathrm{cm}{ }^{-2} \\
(21)\end{array}$ \\
\hline 35.67 & -0.04 & 51.9 & ab09 & 0.40 & 4.86 & was +03 & & & & kfar & ab09 & 10.4 & 6.1 & 48.4 & 9.8 & 5.7 & 46.5 & 10.4 & 6.0 & 48.4 \\
\hline 36.29 & 0.73 & 76.5 & kb94 & 3.00 & 2.70 & $\mathrm{pbd}+03$ & & & & knear & kb94 & 5.1 & 5.3 & 57.8 & 4.7 & 5.0 & 54.7 & 4.7 & 5.5 & 54.7 \\
\hline 36.30 & -1.70 & 63.5 & $\mathrm{pbd}+03$ & 10.00 & 2.70 & $\mathrm{pbd}+03$ & 1.8 & 0.5 & rus03 & knear & pdd04 & 4.2 & 5.7 & 43.1 & & & 43.1 & & & 43.1 \\
\hline 36.40 & 0.02 & 53.3 & was +03 & 0.08 & 4.86 & was +03 & & & & knear & was +03 & 3.5 & 6.0 & 13.7 & 3.3 & 5.7 & 13.2 & 3.4 & 6.1 & 13.4 \\
\hline 36.50 & -0.20 & 72.7 & $\mathrm{pbd}+03$ & 1.30 & 2.70 & $\mathrm{pbd}+03$ & & & & kfar & pdd04 & 8.9 & 5.5 & 63.4 & 8.4 & 5.1 & 61.0 & 9.0 & 5.5 & 63.9 \\
\hline 37.37 & -0.24 & 39.4 & was +03 & 0.26 & 4.86 & was +03 & & & & kfar & was +03 & 10.9 & 6.6 & 43.3 & 10.2 & 6.2 & 41.4 & 10.7 & 6.5 & 42.8 \\
\hline 37.40 & -0.10 & 54.1 & $\mathrm{pbd}+03$ & 3.90 & 2.70 & $\mathrm{pbd}+03$ & & & & kfar & pdd04 & 9.9 & 6.1 & 98.1 & 9.3 & 5.7 & 94.1 & 9.9 & 6.0 & 98.1 \\
\hline 37.40 & -0.00 & 54.1 & $\mathrm{pbd}+03$ & 4.60 & 2.70 & $\mathrm{pbd}+03$ & & & & kfar & pdd04 & 9.9 & 6.1 & 103.7 & 9.3 & 5.7 & 99.5 & 9.9 & 6.0 & 103.7 \\
\hline 37.50 & -0.10 & 49.0 & $\mathrm{pbd}+03$ & 11.10 & 2.70 & $\mathrm{pbd}+03$ & & & & kfar & pdd04 & 10.2 & 6.2 & 141.9 & 9.6 & 5.9 & 136.3 & 10.2 & 6.2 & 141.9 \\
\hline 37.60 & -0.10 & 53.8 & $\mathrm{pbd}+03$ & 2.10 & 2.70 & pbd +03 & & & & kfar & pdd04 & 9.9 & 6.1 & 79.8 & 9.3 & 5.7 & 76.5 & 9.9 & 6.1 & 79.8 \\
\hline 37.67 & 0.13 & 88.9 & ab09 & 3.70 & 2.70 & pbd +03 & & & & kfar & ab09 & 7.8 & 5.1 & 82.3 & 7.2 & 4.8 & 78.0 & 7.2 & 5.2 & 78.0 \\
\hline 37.70 & -0.10 & 57.8 & $\mathrm{pbd}+03$ & 0.60 & 2.70 & pbd +03 & & & & kfar & pdd04 & 9.6 & 6.0 & 51.5 & 9.1 & 5.6 & 49.7 & 9.6 & 5.9 & 51.5 \\
\hline 37.90 & -0.40 & 59.1 & $\mathrm{pbd}+03$ & 5.90 & 2.70 & $\mathrm{pbd}+03$ & & & & kfar & pdd04 & 9.5 & 5.9 & 109.6 & 8.9 & 5.6 & 104.9 & 9.5 & 5.9 & 109.6 \\
\hline 3.10 & -0.00 & 58.3 & $\mathrm{pbd}+03$ & 0.50 & 2.70 & pbd +03 & & & & kfar & pdd04 & 9.5 & 6.0 & 48.1 & 8.9 & 5.6 & 46.1 & 9.5 & 6.0 & 48.1 \\
\hline 39.25 & -0.07 & 23.0 & was +03 & 0.67 & 4.86 & was +03 & & & & kfar & was +03 & 11.6 & 7.4 & 61.8 & 10.8 & 6.9 & 58.9 & 11.3 & 7.2 & 60.7 \\
\hline 39.90 & -1.30 & 44.3 & $\mathrm{pbd}+03$ & 3.00 & 2.70 & $\mathrm{pbd}+03$ & 2.1 & 0.6 & rus03 & knear & pdd04 & 3.0 & 6.5 & 32.0 & & & 32.0 & & & 32.0 \\
\hline 40.42 & 0.70 & 20.1 & was +03 & 0.07 & 4.86 & was +03 & & & & kfar & was +03 & 11.6 & 7.5 & 29.1 & 10.8 & 7.0 & 27.7 & 11.3 & 7.3 & 28.6 \\
\hline 40.50 & 2.50 & 23.0 & $\mathrm{pbd}+03$ & 8.80 & 2.70 & $\mathrm{pbd}+03$ & & & & knear & pdd04 & 1.6 & 7.4 & 38.2 & 1.6 & 6.9 & 38.2 & 1.7 & 7.3 & 39.8 \\
\hline 40.95 & -0.57 & 60.9 & ab09 & 0.90 & 2.70 & pbd +03 & & & & knear & ab09 & 4.2 & 6.0 & 34.0 & 4.0 & 5.6 & 32.9 & 4.1 & 6.1 & 33.5 \\
\hline 41.10 & -0.20 & 59.4 & $\mathrm{pbd}+03$ & 4.90 & 2.70 & $\mathrm{pbd}+03$ & & & & kfar & pdd04 & 8.7 & 6.0 & 97.2 & 8.2 & 5.7 & 93.4 & 8.7 & 6.0 & 97.2 \\
\hline 41.20 & 0.40 & 71.3 & $\mathrm{pbd}+03$ & 1.00 & 2.70 & $\mathrm{pbd}+03$ & & & & knear & pdd04 & 5.4 & 5.7 & 41.6 & 5.0 & 5.4 & 39.5 & 4.9 & 5.8 & 39.0 \\
\hline 41.50 & 0.00 & 17.5 & $\mathrm{pbd}+03$ & 1.00 & 2.70 & $\mathrm{pbd}+03$ & & & & kfar & pdd04 & 11.6 & 7.7 & 69.3 & 10.8 & 7.1 & 66.1 & 11.2 & 7.4 & 67.7 \\
\hline 41.74 & 0.10 & 11.1 & ahck02 & 0.34 & 4.86 & ahck02 & & & & kfar & ahck02 & 12.0 & 8.0 & 50.4 & 11.2 & 7.4 & 48.1 & 11.6 & 7.7 & 49.3 \\
\hline 41.91 & -0.12 & 18.1 & ab09 & 0.04 & 4.85 & GB6 & & & & kfar & ab09 & 11.4 & 7.7 & 23.9 & 10.7 & 7.1 & 22.9 & 11.1 & 7.4 & 23.5 \\
\hline 42.10 & -0.60 & 66.0 & $\mathrm{pbd}+03$ & 3.40 & 2.70 & $\mathrm{pbd}+03$ & & & & knear & pdd04 & 4.9 & 5.9 & 58.7 & 4.6 & 5.5 & 56.3 & 4.6 & 6.0 & 56.3 \\
\hline 42.11 & -0.44 & 53.4 & was +03 & 0.20 & 4.86 & was +03 & & & & kfar & & 8.9 & & 34.6 & 8.3 & 5.9 & 33.0 & 8.8 & 6.2 & 34.3 \\
\hline 42.40 & -0.38 & 65.0 & ab09 & 0.04 & 4.85 & GB6 & & & & knear & ab09 & 4.8 & 5.9 & 13.4 & 4.5 & 5.6 & 12.8 & 4.5 & 6.0 & 12.8 \\
\hline 43.17 & 0.00 & 7.4 & was +03 & 13.96 & 4.86 & was +03 & & & & kfar & was +03 & 12.0 & 8.2 & 174.0 & 11.1 & 7.6 & 165.2 & 11.5 & 7.9 & 169.1 \\
\hline 43.18 & -0.52 & 59.1 & ab09 & 1.20 & 2.70 & $\mathrm{pbd}+03$ & & & & kfar & ab09 & 8.1 & 6.1 & 58.0 & 7.6 & 5.7 & 55.6 & 8.1 & 6.1 & 58.0 \\
\hline 43.24 & -0.05 & 9.4 & was +03 & 0.79 & 4.86 & was +03 & & & & kfar & was +03 & 11.8 & 8.1 & 66.1 & 11.0 & 7.5 & 63.1 & 11.4 & 7.8 & 64.6 \\
\hline 43.79 & -0.12 & 43.3 & was +03 & 0.11 & 4.86 & was +03 & & & & kfar & was +03 & 9.2 & 6.7 & 29.0 & 8.6 & 6.2 & 27.7 & 9.1 & 6.6 & 28.8 \\
\hline 43.89 & -0.78 & 53.4 & ahck02 & 0.66 & 4.86 & ahck02 & & & & kfar & ahck02 & 8.4 & 6.3 & 49.6 & 7.9 & 5.9 & 47.6 & 8.3 & 6.3 & 49.2 \\
\hline 44.26 & 0.10 & 59.6 & ab09 & 1.20 & 2.70 & $\mathrm{pbd}+03$ & & & & kfar & ab09 & 7.7 & 6.1 & 56.0 & 7.2 & 5.8 & 53.5 & 7.7 & 6.2 & 56.0 \\
\hline 44.79 & -0.49 & 44.8 & ab09 & 1.00 & 2.70 & $\mathrm{pbd}+03$ & & & & kfar & ab09 & 8.9 & 6.6 & 58.1 & 8.3 & 6.2 & 55.5 & 8.7 & 6.6 & 57.2 \\
\hline 45.07 & 0.13 & 59.2 & ab09 & 0.47 & 4.86 & & & & & kfar & ab09 & 7.4 & 6.2 & 40.7 & 6.9 & 5.8 & 38.8 & 7.4 & 6.2 & 40.7 \\
\hline 45.12 & 0.13 & 56.7 & ab09 & 4.28 & 4.86 & ahck02 & & & & kfar & ab09 & 7.7 & 6.2 & 87.3 & 7.2 & 5.9 & 83.5 & 7.7 & 6.3 & 87.3 \\
\hline 45.45 & 0.06 & 55.6 & ab09 & 4.77 & 4.86 & ahck02 & & & & kfar & ab09 & 7.7 & 6.3 & 90.5 & 7.2 & 5.9 & 86.5 & 7.7 & 6.3 & 90.5 \\
\hline 45.47 & 0.05 & 60.1 & ahck02 & 0.75 & 4.86 & ahck02 & & & & ktan & ahck02 & 6.0 & 6.1 & 41.4 & & & & & & \\
\hline 45.82 & -0.29 & 61.2 & ab09 & 0.43 & 4.86 & was +03 & & & & kfar & ab09 & 6.6 & 6.1 & 36.6 & 6.2 & 5.8 & 35.1 & 6.8 & 6.2 & 37.3 \\
\hline 45.93 & -0.40 & 63.9 & ab09 & 0.11 & 4.86 & was +03 & & & & kfar & ab09 & 6.7 & 6.1 & 23.5 & 6.3 & 5.7 & 22.6 & 5.8 & 6.1 & 21.3 \\
\hline 46.50 & -0.20 & 57.2 & $\mathrm{pbd}+03$ & 5.40 & 2.70 & $\mathrm{pbd}+03$ & & & & knear & pdd04 & 4.7 & 6.3 & 66.6 & 4.5 & 5.9 & 64.7 & 4.5 & 6.3 & 64.7 \\
\hline 48.60 & 0.00 & 19.1 & $d+03$ & 13.00 & 2.70 & pbd +03 & & & & kfar & pdd04 & 9.9 & 7.7 & 146.6 & 9.2 & 7.2 & 139.6 & 9.6 & 7.5 & 143.6 \\
\hline 48.60 & 0.20 & 10.7 & $\mathrm{pbd}+03$ & 2.70 & 2.70 & $\mathrm{pbd}+03$ & & & & kfar & pdd04 & 10.6 & 8.1 & 90.9 & 9.8 & 7.5 & 86.3 & 10.1 & 7.8 & 88.0 \\
\hline 48.93 & -0.29 & 66.5 & ab09 & 23.70 & 2.70 & $\mathrm{pbd}+03$ & & & & kfar & ab09 & 7.6 & 6.1 & 150.2 & 7.2 & 5.7 & 144.9 & 5.5 & 6.4 & 121.1 \\
\hline 49.00 & -0.30 & 64.8 & $\mathrm{pbd}+03$ & 113.00 & 2.70 & $\mathrm{pbd}+03$ & 5.1 & 2.2 & $\mathrm{xrm}+08$ & & & & & 193.7 & & & 193.7 & & & 193.7 \\
\hline 49.10 & -0.70 & & $\mathrm{pbd}+03$ & 34.00 & 2.70 & $\mathrm{pbd}+03$ & 5.1 & 2.2 & $\mathrm{xrm}+08$ & & & & & 129.8 & & & 129.8 & & & 129.8 \\
\hline 49.10 & -0.40 & 71.2 & $\mathrm{pbd}+03$ & 11.20 & 2.70 & $\mathrm{pbd}+03$ & 5.1 & 2.2 & $\mathrm{xrm}+08$ & & & & & 89.6 & & & 89.6 & & & 89.6 \\
\hline 49.20 & -0.30 & 68.6 & pbd +03 & 23.80 & 2.70 & pbd +03 & 5.1 & 2.2 & $\mathrm{xrm}+08$ & & & & & 115.3 & & & 115.3 & & & 115.3 \\
\hline 49.50 & -0.40 & 57.5 & $\mathrm{pbd}+03$ & 119.00 & 2.70 & $\mathrm{pbd}+03$ & 5.1 & 2.2 & $\mathrm{xrm}+08$ & & & & & 197.1 & & & 197.1 & & & 197.1 \\
\hline 51.20 & -0.10 & & $\mathrm{pbd}+03$ & 39.40 & 2.70 & $\mathrm{pbd}+03$ & 5.1 & 2.2 & $\mathrm{xrm}+08$ & & & & & 136.3 & & & 136.3 & & & 136.3 \\
\hline 49.06 & -0.26 & 64.1 & ab09 & 1.10 & 2.70 & $\mathrm{pbd}+03$ & & & & knear & ab09 & 3.7 & 6.2 & 33.4 & 3.5 & 5.8 & 32.2 & 5.5 & 6.4 & 43.5 \\
\hline 49.37 & -0.30 & 53.7 & $\mathrm{kjb}+03$ & 28.60 & 2.70 & $\mathrm{pbd}+03$ & & & & kfar & $\mathrm{kjb}+03$ & 5.7 & 6.5 & 132.0 & 5.7 & 6.1 & 132.0 & 5.8 & 6.5 & 133.5 \\
\hline 49.40 & -0.20 & 48.0 & $\mathrm{pbd}+03$ & 5.00 & 2.70 & $\mathrm{pbd}+03$ & & & & kfar & pdd04 & 7.1 & 6.6 & 85.4 & 6.5 & 6.2 & 80.5 & 6.9 & 6.6 & 83.8 \\
\hline 49.44 & -0.47 & 60.5 & ab09 & 4.90 & 2.70 & $\mathrm{pbd}+03$ & & & & kfar & ab09 & 7.1 & 6.3 & 84.8 & 6.8 & 5.9 & 82.4 & 5.5 & 6.5 & 71.5 \\
\hline 49.58 & -0.38 & 62.1 & ab09 & 7.60 & 2.70 & $\mathrm{pbd}+03$ & & & & knear & ab09 & 3.7 & 6.2 & 63.6 & 3.5 & 5.8 & 61.3 & 5.4 & 6.5 & 81.8 \\
\hline 49.59 & -0.46 & 64.2 & ab09 & 1.90 & 2.70 & $\mathrm{pbd}+03$ & & & & knear & ab09 & 3.5 & 6.2 & 38.6 & 3.3 & 5.8 & 37.1 & 5.4 & 6.5 & 51.5 \\
\hline 49.67 & -0.45 & 62.7 & was +03 & 0.08 & 4.86 & was +03 & & & & ktan & was +03 & 5.5 & 6.5 & 18.5 & 5.2 & 6.1 & 17.8 & 5.4 & 6.4 & 18.3 \\
\hline 49.70 & -0.17 & 57.0 & ab09 & 0.03 & 4.85 & GB6 & & & & ktan & ab09 & 5.5 & 6.5 & 13.4 & 5.2 & 6.1 & 12.9 & 5.4 & 6.4 & 13.2 \\
\hline
\end{tabular}


Table A.1. continued.

\begin{tabular}{|c|c|c|c|c|c|c|c|c|c|c|c|c|c|c|c|c|c|c|c|c|}
\hline $\begin{array}{r}l \\
\left({ }^{\circ}\right) \\
(1) \\
\end{array}$ & $\begin{array}{r}b \\
\left({ }^{\circ}\right) \\
(2) \\
\end{array}$ & $\begin{array}{c}V_{\mathrm{lsr}} \\
\mathrm{km} \mathrm{s}^{-1} \\
(3)\end{array}$ & Ref. & $\begin{array}{c}S \\
\text { Jy } \\
(5)\end{array}$ & $\begin{array}{r}\text { Freq } \\
\text { GHz } \\
(6)\end{array}$ & Ref. & $\begin{array}{r}d \\
\mathrm{kpc} \\
(8)\end{array}$ & $\begin{array}{r}d_{\text {erro }} \\
\text { kpc } \\
(9)\end{array}$ & (10) & Mark & (12) & $\begin{array}{r}D_{8.5} \\
\mathrm{kpc} \\
(13)\end{array}$ & $\begin{array}{r}R_{8.5} \\
\mathrm{kpc} \\
(14)\end{array}$ & $\begin{array}{r}U_{8.5} \\
\mathrm{pc} \mathrm{cm}^{-2} \\
(15) \\
\end{array}$ & $\begin{array}{r}D_{8.0} \\
\mathrm{kpc} \\
(16)\end{array}$ & $\begin{array}{l}R_{8.0} \\
\mathrm{kpc} \\
(17)\end{array}$ & $\begin{array}{r}U_{8.0} \\
\mathrm{pc} \mathrm{cm}^{-2} \\
(18)\end{array}$ & $\begin{array}{c}D_{8.4} \\
\mathrm{kpc} \\
(19)\end{array}$ & $\begin{array}{l}R_{8.4} \\
\mathrm{kpc} \\
(20)\end{array}$ & $\begin{array}{r}U_{8.4} \\
\mathrm{pc} \mathrm{cm}^{-2} \\
(21)\end{array}$ \\
\hline$\overline{50.10}$ & -0.70 & 72.0 & $\mathrm{pbd}+03$ & 4.00 & 2.70 & $\mathrm{pbd}+03$ & & & & ktan & hh09 & 5.5 & 6.5 & 66.9 & 5.1 & 6.1 & 63.6 & 5.4 & 6.4 & 66.1 \\
\hline 50.20 & 0.30 & 71.2 & $\mathrm{pbd}+03$ & 0.80 & 2.70 & $\mathrm{pbd}+03$ & & & & ktan & hh09 & 5.4 & 6.5 & 38.6 & 5.1 & 6.1 & 37.2 & 5.4 & 6.5 & 38.6 \\
\hline 50.28 & -0.39 & 10.3 & was +03 & 0.27 & 4.86 & was +03 & & & & kfar & was +03 & 10.2 & 8.1 & 41.9 & 9.5 & 7.5 & 40.0 & 9.8 & 7.8 & 40.8 \\
\hline 50.29 & 0.69 & 25.9 & pmg08 & 0.27 & 1.40 & $\mathrm{ccg}+98$ & & & & kfar & pmg08 & 9.0 & 7.4 & 37.0 & 8.3 & 6.9 & 35.1 & 8.6 & 7.3 & 35.9 \\
\hline 51.10 & 0.20 & 42.9 & $\mathrm{pbd}+03$ & 5.90 & 2.70 & $\mathrm{pbd}+03$ & & & & kfar & pdd04 & 7.1 & 6.8 & 90.3 & 6.5 & 6.4 & 85.1 & 6.8 & 6.8 & 87.7 \\
\hline 51.17 & -0.74 & 44.3 & ab09 & 0.04 & 4.85 & GB6 & & & & kfar & ab09 & 6.9 & 6.8 & 17.1 & 6.3 & 6.4 & 16.1 & 6.6 & 6.7 & 16.6 \\
\hline 51.20 & 0.10 & 55.3 & $\mathrm{pbd}+03$ & 41.00 & 2.70 & $\mathrm{pbd}+03$ & & & & ktan & hh09 & 5.3 & 6.6 & 141.7 & 5.0 & 6.2 & 136.3 & 5.3 & 6.6 & 141.7 \\
\hline 1.36 & 0.00 & 52.7 & ab09 & 4.00 & 2.70 & $\mathrm{pbd}+03$ & & & & kfar & ab09 & 6.5 & 6.5 & 74.8 & 6.2 & 6.1 & 72.5 & 5.2 & 6.6 & 64.5 \\
\hline 52.20 & 0.70 & 2.2 & $\mathrm{pbd}+03$ & 2.00 & 2.70 & $\mathrm{pbd}+03$ & & & & kout & hh09 & 10.4 & 8.5 & 81.2 & & & & 9.9 & 8.2 & 78.6 \\
\hline 52.20 & -0.50 & 47.6 & $\mathrm{pbd}+03$ & 3.00 & 2.70 & $\mathrm{pbd}+03$ & & & & ktan & hh09 & 5.2 & 6.7 & 58.5 & 4.9 & 6.3 & 56.2 & 5.2 & 6.6 & 58.5 \\
\hline 52.23 & 0.74 & -3.0 & was +03 & 0.24 & 4.86 & was +03 & & & & kfar & was +03 & 10.9 & 8.8 & 42.1 & 10.0 & 8.1 & 39.7 & 10.2 & 8.4 & 40.3 \\
\hline 52.80 & 0.30 & 16.6 & $\mathrm{pbd}+03$ & 0.50 & 2.70 & $\mathrm{pbd}+03$ & & & & kfar & pdd04 & 9.1 & 7.9 & 46.8 & 8.4 & 7.3 & 44.4 & 8.7 & 7.6 & 45.4 \\
\hline 52.94 & -0.59 & 43.5 & ab09 & 7.00 & 2.70 & $\mathrm{pbd}+03$ & & & & kfar & ab09 & 6.1 & 6.8 & 86.4 & 5.4 & 6.4 & 79.7 & 5.7 & 6.8 & 82.6 \\
\hline 53.18 & 0.16 & 8.3 & kb94 & 9.00 & 2.70 & $\mathrm{pbd}+03$ & & & & kfar & kb94 & 9.7 & 8.2 & 127.9 & 9.0 & 7.6 & 121.7 & 9.2 & 7.9 & 123.5 \\
\hline 53.50 & 0.00 & 24.0 & rus03 & 0.60 & 2.70 & $\mathrm{pbd}+03$ & 1.4 & 0.4 & rus 03 & knear & pdd04 & 1.8 & 7.6 & 14.3 & & & 14.3 & & & 14.3 \\
\hline 53.60 & 0.20 & 38.0 & $\mathrm{pbd}+03$ & 7.00 & 2.70 & $\mathrm{pbd}+03$ & & & & kfar & pdd04 & 6.7 & 7.0 & 91.9 & 6.1 & 6.6 & 86.3 & 6.4 & 7.0 & 89.1 \\
\hline 54.10 & -0.06 & 42.8 & ab09 & 0.34 & 4.86 & was +03 & & & & kfar & ab09 & 5.4 & 6.9 & 29.6 & 5.3 & 6.5 & 29.2 & 4.9 & 6.9 & 27.7 \\
\hline 55.10 & 2.40 & -79.7 & $\mathrm{pbd}+03$ & 1.10 & 2.70 & $\mathrm{pbd}+03$ & & & & kout & hh09 & 19.1 & 15.8 & 99.7 & 17.3 & 14.3 & 93.3 & 16.7 & 13.7 & 91.2 \\
\hline 55.90 & -3.80 & 29.6 & rus03 & & & & 4.5 & 1.3 & rus03 & & & & & & & & & & & \\
\hline 57.55 & -0.27 & 6.3 & was +03 & 0.24 & 4.86 & was +03 & & & & kfar & was +03 & 8.8 & 8.3 & 36.5 & 8.1 & 7.7 & 34.5 & 8.2 & 8.0 & 34.8 \\
\hline 59.50 & -0.20 & 29.4 & $\mathrm{pbd}+03$ & 15.00 & 2.70 & $\mathrm{pbd}+03$ & 2.3 & 0.25 & rus 03 & & & & & 58.1 & & & 58.1 & & & 58.1 \\
\hline 59.60 & 0.92 & 46.8 & was +03 & 0.05 & 4.86 & was +03 & & & & ktan & was +03 & 4.3 & 7.3 & 13.4 & 4.0 & 6.9 & 12.8 & 4.3 & 7.3 & 13.4 \\
\hline 59.80 & 0.20 & -3.3 & $\mathrm{pbd}+03$ & 1.10 & 2.70 & $\mathrm{pbd}+03$ & & & & kout & hh09 & 9.1 & 8.8 & 60.8 & 8.3 & 8.1 & 57.2 & 8.4 & 8.4 & 57.6 \\
\hline 60.90 & -0.10 & 18.6 & $\mathrm{pbd}+03$ & 2.00 & 2.70 & $\mathrm{pbd}+03$ & 2.2 & 0.3 & rus 03 & knear & ahck02 & 1.7 & 7.8 & 28.8 & & & 28.8 & & & 28.8 \\
\hline 61.50 & 0.10 & 27.3 & $\mathrm{pbd}+03$ & 9.00 & 2.70 & $\mathrm{pbd}+03$ & 2.2 & 0.3 & rus 03 & & & & & 47.6 & & & 47.6 & & & 47.6 \\
\hline 62.90 & 0.10 & 19.2 & $\mathrm{pbd}+03$ & 0.60 & 2.70 & $\mathrm{pbd}+03$ & 2.2 & 0.3 & rus 03 & & & & & 19.3 & & & 19.3 & & & 19.3 \\
\hline 62.90 & 0.10 & 19.2 & $\mathrm{pbd}+03$ & 0.30 & 2.70 & $\mathrm{pbd}+03$ & 2.2 & 0.3 & rus 03 & knear & pdd04 & 1.9 & 7.8 & 15.3 & & & 15.3 & & & 15.3 \\
\hline 63.05 & -0.34 & -9.3 & was +03 & 0.04 & 4.86 & was +03 & & & & kfar & was +03 & 8.9 & 9.1 & 20.3 & 8.1 & 8.4 & 19.1 & 8.0 & 8.7 & 18.9 \\
\hline 63.20 & 0.40 & 15.3 & $\mathrm{pbd}+03$ & 5.10 & 2.70 & $\mathrm{pbd}+03$ & & & & knear & pdd04 & 1.4 & 8.0 & 29.1 & 1.6 & 7.4 & 31.8 & 2.0 & 7.8 & 36.9 \\
\hline 63.20 & 0.50 & 19.4 & $\mathrm{~d}+03$ & 7.00 & 2.70 & $\mathrm{pbd}+03$ & 2.2 & 0.3 & rus 03 & knear & pdd04 & 1.9 & 7.8 & 43.8 & & & 43.8 & & & 43.8 \\
\hline 64.10 & -0.50 & 23.6 & $\mathrm{pbd}+03$ & 1.50 & 2.70 & $\mathrm{pbd}+03$ & 3.9 & 0.5 & rus 03 & & & & & 38.4 & & & 38.4 & & & 38.4 \\
\hline 66.80 & 0.90 & 21.0 & $\mathrm{~d}+03$ & 0.60 & 2.70 & $\mathrm{pbd}+03$ & & & & ktan & hh09 & 3.3 & 7.8 & 25.3 & 3.2 & 7.4 & 24.8 & 3.3 & 7.7 & 25.3 \\
\hline 68.10 & 0.90 & -32.5 & $\mathrm{pbd}+03$ & 0.90 & 2.70 & $\mathrm{pbd}+03$ & & & & kout & hh09 & 9.9 & 10.3 & 60.2 & 8.9 & 9.5 & 56.1 & 8.8 & 9.7 & 55.7 \\
\hline 68.20 & 1.00 & & $\mathrm{pbd}+03$ & 10.00 & 2.70 & $\mathrm{pbd}+03$ & 3.6 & 1.1 & rus 03 & & & & & 68.4 & & & 68.4 & & & 68.4 \\
\hline 69.90 & 1.50 & -63.2 & $\mathrm{pbd}+03$ & 4.90 & 2.70 & $\mathrm{pbd}+03$ & & & & kout & hh09 & 12.7 & 12.6 & 125.0 & 11.5 & 11.5 & 117.0 & 11.1 & 11.4 & 114.3 \\
\hline 70.20 & 1.70 & -22.9 & $\mathrm{pbd}+03$ & 2.20 & 2.70 & $\mathrm{pbd}+03$ & 7.0 & 1.5 & rus03 & & & & & 64.4 & & & 64.4 & & & 64.4 \\
\hline 70.29 & 1.60 & -24.2 & ahck02 & 4.37 & 4.86 & ahck02 & 7.0 & 1.5 & rus03 & & & & & 82.5 & & & 82.5 & & & 82.5 \\
\hline 70.33 & 1.59 & -21.1 & ahck02 & 2.21 & 4.86 & ahck02 & 7.0 & 1.5 & rus03 & & & & & 65.7 & & & 65.7 & & & 65.7 \\
\hline 71.60 & 2.80 & 16.0 & $\mathrm{pbd}+03$ & 5.30 & 2.70 & $\mathrm{pbd}+03$ & 2.8 & 0.8 & rus 03 & & & & & 46.8 & & & 46.8 & & & 46.8 \\
\hline 74.80 & 0.60 & -2.2 & $\mathrm{pbd}+03$ & 11.70 & 2.70 & $\mathrm{pbd}+03$ & 3.1 & 0.9 & rus 03 & & & & & 65.3 & & & 65.3 & & & 65.3 \\
\hline 75.40 & -0.40 & -0.8 & $\mathrm{pbd}+03$ & 0.70 & 2.70 & $\mathrm{pbd}+03$ & & & & kout & hh09 & 4.9 & 8.7 & 34.6 & 4.1 & 8.0 & 30.7 & 3.6 & 8.4 & 28.2 \\
\hline 75.80 & 0.30 & -8.5 & $\mathrm{pbd}+03$ & 5.00 & 2.70 & $\mathrm{pbd}+03$ & & & & kout & hh09 & 5.7 & 9.0 & 73.8 & 5.0 & 8.3 & 67.6 & 4.6 & 8.6 & 64.0 \\
\hline 75.80 & 0.40 & -4.2 & $\mathrm{pbd}+03$ & 11.80 & 2.70 & $\mathrm{pbd}+03$ & & & & kout & hh09 & 5.2 & 8.8 & 92.4 & 4.5 & 8.2 & 83.9 & 4.1 & 8.5 & 78.9 \\
\hline 76.20 & -0.30 & -28.2 & $\mathrm{pbd}+03$ & 1.50 & 2.70 & $\mathrm{pbd}+03$ & & & & kout & hh09 & 7.7 & 10.0 & 60.4 & 6.9 & 9.2 & 56.1 & 6.6 & 9.4 & 54.5 \\
\hline 77.40 & -3.70 & 8.1 & rus03 & 0.10 & 2.70 & $\mathrm{pbd}+03$ & 2.6 & 0.8 & rus 03 & & & & & 11.9 & & & 11.9 & & & 11.9 \\
\hline 78.00 & -0.00 & -1.0 & $\mathrm{pbd}+03$ & 17.90 & 2.70 & $\mathrm{pbd}+03$ & & & & kout & hh09 & 4.2 & 8.7 & 92.1 & 3.5 & 8.0 & 81.6 & 2.7 & 8.4 & 68.6 \\
\hline 78.00 & 0.60 & 1.3 & $\mathrm{pbd}+03$ & 9.00 & 2.70 & $\mathrm{pbd}+03$ & & & & kout & hh09 & 3.9 & 8.6 & 69.7 & & & & 1.8 & 8.3 & 41.6 \\
\hline 78.20 & -0.40 & 0.5 & $\mathrm{pbd}+03$ & 44.20 & 2.70 & $\mathrm{pbd}+03$ & & & & kout & 109 & 4.0 & 8.6 & 120.5 & & & & 1.7 & 8.3 & 68.1 \\
\hline 78.30 & 1.40 & -0.9 & +03 & 1.70 & 2.70 & $\mathrm{pbd}+03$ & & & & & hh09 & 4.1 & 8.7 & 41.3 & 3.4 & 8.0 & 36.5 & 2.6 & 8.4 & 30.5 \\
\hline 78.30 & 1.50 & -3.7 & +03 & 3.10 & 2.70 & $\mathrm{pbd}+03$ & & & & kol & hh09 & 4.5 & 8.8 & 53.7 & 3.8 & 8.1 & 48.0 & 3.2 & 8.5 & 42.8 \\
\hline 78.30 & 2.80 & -5.4 & $1+03$ & 3.60 & 2.70 & $\mathrm{pbd}+03$ & & & & ko & hh09 & 4.8 & 8.9 & 59.0 & 4.1 & 8.2 & 53.1 & 3.6 & 8.5 & 48.7 \\
\hline 78.40 & 2.70 & -0.5 & +03 & 1.50 & 2.70 & $\mathrm{pbd}+03$ & & & & & hh09 & 4.1 & 8.7 & 39.7 & 3.3 & 8.0 & 34.4 & 2.4 & 8.4 & 27.8 \\
\hline 78.50 & -0.00 & -7.8 & $d+03$ & 4.90 & 2.70 & $\mathrm{pbd}+03$ & & & & kout & hh09 & 5.0 & 9.0 & 67.2 & 4.3 & 8.3 & 60.8 & 3.8 & 8.6 & 56.0 \\
\hline 78.50 & 1.20 & -4.5 & $d+03$ & 155.20 & 2.70 & $\mathrm{pbd}+03$ & & & & kout & hh09 & 4.6 & 8.8 & 201.0 & 3.9 & 8.2 & 180.1 & 3.3 & 8.5 & 161.1 \\
\hline 78.60 & 0.80 & -4.5 & $\mathrm{pbd}+03$ & 8.00 & 2.70 & $\mathrm{pbd}+03$ & & & & kout & hh09 & 4.6 & 8.8 & 74.8 & 3.9 & 8.2 & 67.0 & 3.3 & 8.5 & 59.9 \\
\hline 78.70 & -0.60 & -7.9 & $\mathrm{pbd}+03$ & 15.50 & 2.70 & $\mathrm{pbd}+03$ & & & & kout & hh09 & 5.0 & 9.0 & 98.6 & 4.3 & 8.3 & 89.2 & 3.8 & 8.6 & 82.1 \\
\hline 78.70 & 0.90 & 3.0 & $\mathrm{pbd}+03$ & 5.90 & 2.70 & $\mathrm{pbd}+03$ & & & & kout & hh09 & 3.4 & 8.5 & 55.3 & & & & 1.6 & 8.3 & 33.5 \\
\hline 79.20 & -0.30 & -3.3 & $\mathrm{pbd}+03$ & 5.00 & 2.70 & $\mathrm{pbd}+03$ & & & & kout & hh09 & 4.3 & 8.8 & 61.1 & 3.6 & 8.1 & 54.3 & 2.8 & 8.4 & 45.9 \\
\hline 79.20 & -0.10 & -5.0 & $\mathrm{pbd}+03$ & 21.80 & 2.70 & $\mathrm{pbd}+03$ & & & & kout & hh09 & 4.5 & 8.8 & 103.0 & 3.8 & 8.2 & 92.0 & 3.2 & 8.5 & 82.1 \\
\hline 79.20 & 0.30 & -3.9 & $\mathrm{pbd}+03$ & 5.30 & 2.70 & $\mathrm{pbd}+03$ & & & & kout & hh09 & 4.4 & 8.8 & 63.3 & 3.6 & 8.1 & 55.4 & 3.0 & 8.5 & 49.0 \\
\hline 79.30 & 1.30 & -41.2 & $\mathrm{pbd}+03$ & 27.50 & 2.70 & $\mathrm{pbd}+03$ & & & & kout & hh09 & 8.4 & 10.8 & 168.7 & 7.5 & 9.9 & 156.4 & 7.1 & 10.0 & 150.8 \\
\hline 79.30 & 0.30 & 8.5 & $\mathrm{pbd}+03$ & 34.30 & 2.70 & $\mathrm{pbd}+03$ & & & & ktan & hh09 & 1.6 & 8.4 & 60.1 & 1.5 & 7.9 & 57.6 & 1.6 & 8.3 & 60.1 \\
\hline 79.40 & -0.80 & 1.6 & $\mathrm{pbd}+03$ & 24.40 & 2.70 & $\mathrm{pbd}+03$ & & & & kout & hh09 & 3.4 & 8.6 & 88.7 & & & & 1.6 & 8.4 & 53.7 \\
\hline 79.40 & 2.40 & 3.0 & $\mathrm{pbd}+03$ & 70.70 & 2.70 & $\mathrm{pbd}+03$ & & & & kout & hh09 & 3.2 & 8.5 & 121.4 & & & & 1.6 & 8.4 & 76.5 \\
\hline
\end{tabular}


Table A.1. continued.

\begin{tabular}{|c|c|c|c|c|c|c|c|c|c|c|c|c|c|c|c|c|c|c|c|c|}
\hline $\begin{array}{r}l \\
\left({ }^{\circ}\right) \\
(1)\end{array}$ & $\begin{array}{r}b \\
\left({ }^{\circ}\right) \\
(2)\end{array}$ & $\begin{array}{c}V_{\mathrm{lsr}} \\
\mathrm{km} \mathrm{s}^{-1} \\
(3)\end{array}$ & Ref. & $\begin{array}{c}S \\
\text { Jy } \\
(5)\end{array}$ & $\begin{array}{r}\text { Freq } \\
\text { GHz } \\
(6)\end{array}$ & Ref. & $\begin{array}{r}d \\
\mathrm{kpc} \\
(8)\end{array}$ & $\begin{array}{r}d_{\text {erro }} \\
\mathrm{kpc} \\
(9)\end{array}$ & Ref. & Mark & Ref. & $\begin{array}{r}D_{8.5} \\
\mathrm{kpc} \\
(13)\end{array}$ & $\begin{array}{r}R_{8.5} \\
\mathrm{kpc} \\
(14)\end{array}$ & $\begin{array}{r}U_{8.5} \\
\mathrm{pc} \mathrm{cm}^{-2} \\
(15)\end{array}$ & $\begin{array}{c}D_{8.0} \\
\mathrm{kpc} \\
(16)\end{array}$ & $\begin{array}{l}R_{8.0} \\
\mathrm{kpc} \\
(17)\end{array}$ & $\begin{array}{r}U_{8.0} \\
\mathrm{pc} \mathrm{cm}^{-2} \\
(18)\end{array}$ & $\begin{array}{c}D_{8.4} \\
\mathrm{kpc} \\
(19)\end{array}$ & $\begin{array}{c}R_{8.4} \\
\mathrm{kpc} \\
(20)\end{array}$ & $\begin{array}{r}U_{8.4} \\
\mathrm{pc} \mathrm{cm}{ }^{-2} \\
(21)\end{array}$ \\
\hline 79.80 & 0.10 & -7.2 & $\mathrm{pbd}+03$ & 1.90 & 2.70 & $\mathrm{pbd}+03$ & & & & kout & hh09 & 4.7 & 8.9 & 47.0 & 4.0 & 8.3 & 42.2 & 3.4 & 8.6 & 37.9 \\
\hline 80.00 & -0.00 & -1.0 & $d+03$ & 4.20 & 2.70 & $\mathrm{pbd}+03$ & & & & kout & hh09 & 3.7 & 8.7 & 52.2 & 3.0 & 8.0 & 45.4 & 1.5 & 8.4 & 28.6 \\
\hline 80.00 & 0.90 & -14.8 & $d+03$ & 3.20 & 2.70 & $\mathrm{pbd}+03$ & & & & kout & hh09 & 5.5 & 9.3 & 62.1 & 4.8 & 8.6 & 56.7 & 4.4 & 8.9 & 53.5 \\
\hline 80.00 & 1.50 & -15.4 & $\mathrm{pbd}+03$ & 4.30 & 2.70 & $\mathrm{pbd}+03$ & & & & kout & hh09 & 5.6 & 9.3 & 69.3 & 4.9 & 8.6 & 63.4 & 4.5 & 8.9 & 59.9 \\
\hline 80.00 & 2.10 & -4.7 & $\mathrm{pbd}+03$ & 28.10 & 2.70 & $\mathrm{pbd}+03$ & & & & kout & hh09 & 4.3 & 8.8 & 108.7 & 3.6 & 8.2 & 96.6 & 3.0 & 8.5 & 85.5 \\
\hline 80.10 & -0.10 & -2.2 & $\mathrm{pbd}+03$ & 66.00 & 2.70 & $\mathrm{pbd}+03$ & & & & kout & hh09 & 3.9 & 8.7 & 135.4 & 3.2 & 8.1 & 118.7 & 2.2 & 8.4 & 92.4 \\
\hline 80.20 & 0.80 & -1.2 & $\mathrm{pbd}+03$ & 2.10 & 2.70 & $\mathrm{pbd}+03$ & & & & kout & hh09 & 3.7 & 8.7 & 41.4 & 3.0 & 8.0 & 36.0 & 1.4 & 8.4 & 21.7 \\
\hline 80.30 & 0.70 & -62.4 & $\mathrm{pbd}+03$ & 4.90 & 2.70 & $\mathrm{pbd}+03$ & & & & kout & hh09 & 10.4 & 12.3 & 109.4 & 9.3 & 11.2 & 101.5 & 8.8 & 11.2 & 97.9 \\
\hline 80.30 & 0.80 & -63.4 & $\mathrm{pbd}+03$ & 25.30 & 2.70 & $\mathrm{pbd}+03$ & & & & kout & hh09 & 10.5 & 12.4 & 190.4 & 9.4 & 11.3 & 176.9 & 8.9 & 11.3 & 170.5 \\
\hline 80.40 & 0.40 & 9.7 & $\mathrm{pbd}+03$ & 53.20 & 2.70 & $\mathrm{pbd}+03$ & & & & ktan & hh09 & 1.4 & 8.4 & 63.6 & 1.3 & 7.9 & 60.5 & 1.4 & 8.3 & 63.6 \\
\hline 80.40 & 0.50 & 7.5 & $\mathrm{pbd}+03$ & 30.80 & 2.70 & $\mathrm{pbd}+03$ & & & & $\mathrm{ktan}$ & hh09 & 1.4 & 8.4 & 53.0 & 1.3 & 7.9 & 50.4 & 1.4 & 8.3 & 53.0 \\
\hline 80.60 & 1.50 & 11.3 & $\mathrm{pbd}+03$ & 15.70 & 2.70 & $\mathrm{pbd}+03$ & & & & ktan & hh09 & 1.4 & 8.4 & 42.4 & 1.3 & 7.9 & 40.4 & 1.4 & 8.3 & 42.4 \\
\hline 80.90 & -0.30 & 0.6 & $\mathrm{pbd}+03$ & 3.90 & 2.70 & $\mathrm{pbd}+03$ & & & & kout & hh09 & 3.2 & 8.6 & 46.2 & & & & 1.3 & 8.4 & 25.3 \\
\hline 80.90 & -0.20 & -1.0 & $\mathrm{pbd}+03$ & 30.20 & 2.70 & $\mathrm{pbd}+03$ & & & & kout & hh09 & 3.5 & 8.7 & 97.1 & 2.7 & 8.0 & 81.7 & 1.3 & 8.4 & 50.2 \\
\hline 80.90 & -0.10 & -2.1 & $\mathrm{pbd}+03$ & 14.00 & 2.70 & $\mathrm{pbd}+03$ & & & & kout & hh09 & 3.7 & 8.7 & 78.0 & 3.0 & 8.1 & 67.8 & 1.7 & 8.4 & 46.4 \\
\hline 80.90 & 0.40 & 1.8 & $\mathrm{pbd}+03$ & 7.90 & 2.70 & $\mathrm{pbd}+03$ & & & & kout & hh09 & 3.0 & 8.6 & 56.0 & & & & 1.3 & 8.4 & 32.1 \\
\hline 81.30 & 1.10 & 12.5 & $\mathrm{pbd}+03$ & 12.30 & 2.70 & $\mathrm{pbd}+03$ & & & & ktan & hh09 & 1.3 & 8.4 & 37.2 & 1.2 & 7.9 & 35.3 & 1.3 & 8.3 & 37.2 \\
\hline 81.30 & 1.20 & 11.4 & $\mathrm{pbd}+03$ & 10.70 & 2.70 & $\mathrm{pbd}+03$ & & & & ktan & hh09 & 1.3 & 8.4 & 35.5 & 1.2 & 7.9 & 33.7 & 1.3 & 8.3 & 35.5 \\
\hline 81.50 & -0.00 & -0.7 & $\mathrm{pbd}+03$ & 6.00 & 2.70 & $\mathrm{pbd}+03$ & & & & kout & hh09 & 3.3 & 8.7 & 54.5 & 2.5 & 8.0 & 45.3 & 1.2 & 8.4 & 27.8 \\
\hline 81.50 & 0.60 & 0.7 & $\mathrm{pbd}+03$ & 78.40 & 2.70 & $\mathrm{pbd}+03$ & & & & kout & hh09 & 3.1 & 8.6 & 123.1 & & & & 1.2 & 8.4 & 65.4 \\
\hline 81.60 & 2.10 & -6.9 & $\mathrm{pbd}+03$ & 3.80 & 2.70 & $\mathrm{pbd}+03$ & & & & kout & hh09 & 4.2 & 8.9 & 54.9 & 3.5 & 8.3 & 48.6 & 2.9 & 8.6 & 42.9 \\
\hline 81.70 & 0.50 & -0.2 & $\mathrm{pbd}+03$ & 15.50 & 2.70 & $\mathrm{pbd}+03$ & & & & kout & hh09 & 3.2 & 8.6 & 73.2 & 2.4 & 8.0 & 60.4 & 1.2 & 8.4 & 38.1 \\
\hline 81.70 & 0.30 & 10.5 & $\mathrm{pbd}+03$ & 0.20 & 2.70 & $\mathrm{pbd}+03$ & & & & ktan & hh09 & 1.2 & 8.4 & 8.9 & 1.2 & 7.9 & 8.9 & 1.2 & 8.3 & 8.9 \\
\hline 81.87 & 0.78 & 1.7 & pmg08 & 0.09 & 1.40 & $\mathrm{ccg}+98$ & & & & kfar & pmg08 & 2.8 & 8.6 & 11.8 & 1.7 & 7.9 & 8.5 & 1.2 & 8.4 & 6.7 \\
\hline 82.00 & 2.30 & -5.1 & $\mathrm{pbd}+03$ & 1.90 & 2.70 & $\mathrm{pbd}+03$ & & & & kout & hh09 & 3.9 & 8.8 & 41.5 & 3.2 & 8.2 & 36.4 & 2.5 & 8.5 & 30.9 \\
\hline 82.30 & 2.40 & -3.3 & $\mathrm{pbd}+03$ & 104.90 & 2.70 & $\mathrm{pbd}+03$ & & & & kout & hh09 & 3.6 & 8.8 & 149.8 & 2.8 & 8.1 & 126.7 & 1.9 & 8.5 & 97.8 \\
\hline 82.40 & 2.50 & -4.6 & $\mathrm{pbd}+03$ & 18.30 & 2.70 & $\mathrm{pbd}+03$ & & & & kout & hh09 & 3.7 & 8.8 & 85.2 & 3.0 & 8.2 & 74.1 & 2.2 & 8.5 & 60.2 \\
\hline 82.60 & 0.40 & -9.6 & $d+03$ & 25.30 & 2.70 & $\mathrm{pbd}+03$ & & & & kout & hh09 & 4.4 & 9.0 & 106.6 & 3.7 & 8.4 & 95.0 & 3.1 & 8.7 & 84.4 \\
\hline 83.80 & 3.30 & 5.9 & $\mathrm{pbd}+03$ & 8.00 & 2.70 & $\mathrm{pbd}+03$ & & & & ktan & hh09 & 0.9 & 8.5 & 25.2 & 0.9 & 8.0 & 25.2 & 0.9 & 8.4 & 25.2 \\
\hline 84.80 & 3.80 & -9.6 & $\mathrm{pbd}+03$ & 11.60 & 2.70 & $\mathrm{pbd}+03$ & & & & kout & hh09 & 4.0 & 9.0 & 77.1 & 3.3 & 8.4 & 67.8 & 2.7 & 8.7 & 59.3 \\
\hline 84.80 & -3.50 & 5.2 & $\mathrm{pbd}+03$ & 0.10 & 2.70 & $\mathrm{pbd}+03$ & & & & $\mathrm{ktan}$ & hh09 & 0.8 & 8.5 & 5.4 & 0.7 & 8.0 & 4.9 & 0.8 & 8.4 & 5.4 \\
\hline 84.90 & -1.20 & 5.5 & $\mathrm{pbd}+03$ & 0.50 & 2.70 & $\mathrm{pbd}+03$ & & & & $\mathrm{ktan}$ & hh09 & 0.8 & 8.5 & 9.2 & 0.7 & 8.0 & 8.4 & 0.8 & 8.4 & 9.2 \\
\hline 85.20 & -0.10 & -0.9 & $\mathrm{pbd}+03$ & 1.50 & 2.70 & $\mathrm{pbd}+03$ & & & & kout & hh09 & 2.6 & 8.7 & 29.3 & 1.7 & 8.0 & 22.1 & 0.7 & 8.5 & 12.2 \\
\hline 85.20 & 0.00 & -31.2 & $\mathrm{pbd}+03$ & 1.50 & 2.70 & $\mathrm{pbd}+03$ & & & & kout & hh09 & 6.3 & 10.1 & 52.8 & 5.5 & 9.3 & 48.2 & 5.1 & 9.5 & 45.9 \\
\hline 85.50 & -1.00 & 3.4 & rus03 & & & & 1.0 & 0.3 & rus03 & & & & & 116.0 & & & 116.0 & & & 116.0 \\
\hline 87.60 & -3.80 & 2.8 & rus 03 & & & & 0.9 & 0.3 & rus03 & & & & & 47.0 & & & 47.0 & & & 47.0 \\
\hline 90.20 & 1.70 & -60.9 & $\mathrm{pbd}+03$ & 1.00 & 2.70 & $\mathrm{pbd}+03$ & & & & kout & hh09 & 8.6 & 12.1 & 56.8 & 7.6 & 11.1 & 52.3 & 7.0 & 11.1 & 49.5 \\
\hline 90.20 & 2.10 & -65.6 & $\mathrm{pbd}+03$ & 0.10 & 2.70 & $\mathrm{pbd}+03$ & & & & kout & hh09 & 9.1 & 12.5 & 27.4 & 8.1 & 11.4 & 25.4 & 7.5 & 11.3 & 24.1 \\
\hline 93.10 & 2.80 & -61.0 & $\mathrm{pbd}+03$ & 2.80 & 2.70 & $\mathrm{pbd}+03$ & 6.3 & 1.1 & rus03 & & & & & 65.0 & & & 65.0 & & & 65.0 \\
\hline 94.40 & -5.50 & 0.9 & $\mathrm{pbd}+03$ & 1.90 & 2.70 & $\mathrm{pbd}+03$ & 0.9 & 0.04 & rus 03 & & & & & 15.6 & & & 15.6 & & & 15.6 \\
\hline 94.50 & -1.50 & -38.6 & rus03 & 2.30 & 2.70 & $\mathrm{pbd}+03$ & 3.6 & 0.8 & rus03 & & & & & 41.9 & & & 41.9 & & & 41.9 \\
\hline 95.40 & 16.80 & -4.0 & rus 03 & & & & 0.4 & 0.04 & rus03 & & & & & & & & & & & \\
\hline 96.00 & 2.00 & -74.0 & rus 03 & & & & 9.6 & 0.4 & rag07 & & & & & 74.7 & & & 74.7 & & & 74.7 \\
\hline 96.30 & 2.60 & -95.0 & rus03 & 0.60 & 2.70 & $\mathrm{pbd}+03$ & 9.7 & 2.9 & rus03 & & & & & 51.9 & & & 51.9 & & & 51.9 \\
\hline 96.30 & -0.20 & -54.2 & rus 03 & & & & 8.88 & 0.61 & rag07 & & & & & 45.1 & & & 45.1 & & & 45.1 \\
\hline 97.60 & 3.20 & -72.5 & $\mathrm{pbd}+03$ & 0.70 & 2.70 & $\mathrm{pbd}+03$ & & & & kout & hh09 & 9.0 & 13.2 & 52.0 & 7.9 & 12.0 & 47.7 & 7.2 & 11.9 & 44.8 \\
\hline 99.10 & 7.40 & -13.9 & $\mathrm{pbd}+03$ & 0.20 & 2.70 & $\mathrm{pbd}+03$ & & & & kout & hh09 & 2.5 & 9.3 & 14.6 & 2.0 & 8.6 & 12.6 & 1.5 & 8.9 & 10.4 \\
\hline 99.30 & 3.70 & -2.0 & rus03 & & & & 0.9 & 0.04 & rus03 & & & & & 59.0 & & & 59.0 & & & 59.0 \\
\hline 100.00 & 10.00 & -9.6 & $\mathrm{~s} 03$ & & & & 0.6 & 0.1 & rus03 & & & & & & & & & & & \\
\hline 101.40 & 2.70 & -61.0 & rus03 & 0.20 & 2.70 & $\mathrm{pbd}+03$ & 5.25 & 0.29 & rag07 & kout & hh09 & 7.2 & 12.2 & 23.7 & & & 23.7 & & & 23.7 \\
\hline 102.90 & -0.70 & -48.1 & $\mathrm{pbd}+03$ & 2.60 & 2.70 & $\mathrm{pbd}+03$ & 3.1 & 0.3 & rus03 & & & & & 39.5 & & & 39.5 & & & 39.5 \\
\hline 103.00 & -0.80 & -48.5 & $\mathrm{pbd}+03$ & 2.70 & 2.70 & $\mathrm{pbd}+03$ & & & & kout & hh09 & 5.7 & 11.3 & 60.1 & 5.0 & 10.3 & 55.1 & 4.4 & 10.4 & 50.6 \\
\hline 103.70 & 2.20 & -16.1 & $\mathrm{pbd}+03$ & 0.60 & 2.70 & $\mathrm{pbd}+03$ & 1.2 & 0.25 & rus03 & & & & & 12.9 & & & 12.9 & & & 12.9 \\
\hline 104.60 & 1.40 & -20.7 & $\mathrm{pbd}+03$ & 1.40 & 2.70 & $\mathrm{pbd}+03$ & 1.2 & 0.25 & rus03 & & & & & 17.1 & & & 17.1 & & & 17.1 \\
\hline 105.20 & 7.10 & -10.3 & $\mathrm{pbd}+03$ & 0.20 & 2.70 & $\mathrm{pbd}+03$ & 0.7 & 0.1 & rus03 & & & & & 6.2 & & & 6.2 & & & 6.2 \\
\hline 105.60 & 0.40 & -52.0 & rus03 & 0.70 & 2.70 & $\mathrm{pbd}+03$ & 2.91 & 1.4 & rag07 & & & & & 24.4 & & & 24.4 & & & 24.4 \\
\hline 106.80 & 3.30 & -65.0 & $\mathrm{pbd}+03$ & 0.60 & 2.70 & $\mathrm{pbd}+03$ & 8.34 & 0.6 & rag07 & & & & & 46.8 & & & 46.8 & & & 46.8 \\
\hline 106.80 & 5.30 & -8.5 & $\mathrm{pbd}+03$ & 0.70 & 2.70 & $\mathrm{pbd}+03$ & 0.9 & 0.1 & rus03 & & & & & 11.2 & & & 11.2 & & & 11.2 \\
\hline 107.20 & -1.00 & -36.0 & $\mathrm{pbd}+03$ & 6.60 & 2.70 & $\mathrm{pbd}+03$ & 3.7 & 0.25 & rus03 & & & & & 60.7 & & & 60.7 & & & 60.7 \\
\hline 107.30 & -0.90 & -41.0 & $\mathrm{pbd}+03$ & 0.60 & 2.70 & $\mathrm{pbd}+03$ & & & & kout & hh09 & 4.6 & 10.8 & 31.5 & 4.0 & 9.9 & 28.7 & 3.4 & 10.1 & 25.8 \\
\hline 108.20 & 0.60 & -56.3 & $\mathrm{pbd}+03$ & 1.50 & 2.70 & $\mathrm{pbd}+03$ & & & & kout & hh09 & 6.2 & 12.0 & 52.2 & 5.4 & 10.9 & 47.6 & 4.8 & 11.0 & 44.0 \\
\hline 108.20 & 5.60 & -8.8 & $\mathrm{pbd}+03$ & 0.10 & 2.70 & $\mathrm{pbd}+03$ & 0.9 & 0.1 & rus03 & & & & & 5.9 & & & 5.9 & & & 5.9 \\
\hline 108.30 & -1.00 & -55.2 & rus03 & 0.60 & 2.70 & $\mathrm{pbd}+03$ & 5.3 & 1.1 & rus03 & & & & & 34.7 & & & 34.7 & & & 34.7 \\
\hline 108.80 & -0.90 & -50.1 & $\mathrm{pbd}+03$ & 0.90 & 2.70 & $\mathrm{pbd}+03$ & 2.39 & 0.21 & rag07 & & & & & 22.7 & & & 22.7 & & & 22.7 \\
\hline
\end{tabular}


Table A.1. continued.

\begin{tabular}{|c|c|c|c|c|c|c|c|c|c|c|c|c|c|c|c|c|c|c|c|c|}
\hline $\begin{array}{r}l \\
\left({ }^{\circ}\right) \\
(1) \\
\end{array}$ & $\begin{array}{l}b \\
\left({ }^{\circ}\right) \\
(2)\end{array}$ & $\begin{array}{c}V_{\mathrm{lsr}} \\
\mathrm{km} \mathrm{s}^{-1} \\
(3)\end{array}$ & Ref. & $\begin{array}{r}S \\
\text { Jy } \\
(5)\end{array}$ & $\begin{array}{r}\text { Freq } \\
\text { GHz } \\
(6)\end{array}$ & Ref. & $\begin{array}{r}d \\
\mathrm{kpc} \\
(8)\end{array}$ & $\begin{array}{r}d_{\text {erro }} \\
\text { kpc } \\
(9)\end{array}$ & Ref. & Mark & Ref. & $\begin{array}{l}D_{8.5} \\
\mathrm{kpc} \\
(13)\end{array}$ & $\begin{array}{r}R_{8.5} \\
\mathrm{kpc} \\
(14)\end{array}$ & $\begin{array}{r}U_{8.5} \\
\mathrm{pc} \mathrm{cm}^{-2} \\
(15)\end{array}$ & $\begin{array}{r}D_{8.0} \\
\mathrm{kpc} \\
(16)\end{array}$ & $\begin{array}{l}R_{8.0} \\
\mathrm{kpc} \\
(17)\end{array}$ & $\begin{array}{r}U_{8.0} \\
\mathrm{pc} \mathrm{cm}^{-2} \\
(18)\end{array}$ & $\begin{array}{l}D_{8.4} \\
\mathrm{kpc} \\
(19)\end{array}$ & $\begin{array}{r}R_{8.4} \\
\mathrm{kpc} \\
(20)\end{array}$ & $\begin{array}{r}U_{8.4} \\
\mathrm{pc} \mathrm{cm}^{-2} \\
(21)\end{array}$ \\
\hline 108.80 & -1.00 & -49.1 & $\mathrm{pbd}+03$ & 1.80 & 2.70 & $\mathrm{pbd}+03$ & 2.39 & 0.21 & rag07 & & & & & 28.7 & & & 28.7 & & & 28.7 \\
\hline 109.10 & -0.30 & -47.0 & $\mathrm{pbd}+03$ & 0.40 & 2.70 & $\mathrm{pbd}+03$ & & & & kout & hh09 & 5.1 & 11.3 & 29.5 & 4.4 & 10.3 & 26.7 & 3.9 & 10.4 & 24.7 \\
\hline 109.20 & 1.50 & -11.5 & $\mathrm{pbd}+03$ & 0.50 & 2.70 & $\mathrm{pbd}+03$ & 0.7 & 0.04 & rus 03 & & & & & 8.5 & & & 8.5 & & & 8.5 \\
\hline 110.10 & 0.10 & -49.8 & $\mathrm{pbd}+03$ & 2.10 & 2.70 & $\mathrm{pbd}+03$ & & & & kout & hh09 & 5.4 & 11.5 & 53.3 & 4.6 & 10.5 & 47.9 & 4.1 & 10.6 & 44.4 \\
\hline 110.10 & 0.00 & -51.7 & $\mathrm{pbd}+03$ & 2.50 & 2.70 & $\mathrm{pbd}+03$ & 3.0 & 0.7 & rag07 & & & & & 38.2 & & & 38.2 & & & 38.2 \\
\hline 110.20 & 2.50 & -10.0 & $\mathrm{pbd}+03$ & 5.00 & 2.70 & $\mathrm{pbd}+03$ & 0.7 & 0.04 & rus03 & & & & & 18.2 & & & 18.2 & & & 18.2 \\
\hline 111.30 & -0.70 & -43.0 & $\mathrm{pbd}+03$ & 1.20 & 2.70 & $\mathrm{pbd}+03$ & & & & kout & hh09 & 4.6 & 11.0 & 39.7 & 3.9 & 10.1 & 35.6 & 3.4 & 10.2 & 32.5 \\
\hline 111.50 & 0.80 & -60.8 & $\mathrm{pbd}+03$ & 28.60 & 2.70 & $\mathrm{pbd}+03$ & 2.4 & 0.14 & rag07 & & & & & 74.1 & & & 74.1 & & & 74.1 \\
\hline 111.60 & 0.90 & -60.6 & $\mathrm{pbd}+03$ & 0.60 & 2.70 & $\mathrm{pbd}+03$ & 2.65 & 0.1 & $\mathrm{mrm}+08$ & & & & & 21.6 & & & 21.6 & & & 21.6 \\
\hline 111.60 & 0.40 & -64.5 & $\mathrm{pbd}+03$ & 2.20 & 2.70 & $\mathrm{pbd}+03$ & 2.4 & 0.14 & rag07 & & & & & 31.5 & & & 31.5 & & & 31.5 \\
\hline 111.90 & 4.10 & 5.8 & rus03 & & & & 1.1 & 0.3 & rus03 & & & & & & & & & & & \\
\hline 112.20 & 0.20 & -41.8 & $\mathrm{pbd}+03$ & 7.30 & 2.70 & $\mathrm{pbd}+03$ & 3.2 & 0.25 & rus03 & & & & & 57.0 & & & 57.0 & & & 57.0 \\
\hline 113.60 & -0.70 & -42.7 & $\mathrm{pbd}+03$ & 6.90 & 2.70 & $\mathrm{pbd}+03$ & 3.53 & 0.17 & rag07 & & & & & 59.3 & & & 59.3 & & & 59.3 \\
\hline 114.60 & 0.20 & -33.0 & rus03 & & & & 1.7 & 0.5 & rus03 & & & & & 19.0 & & & 19.0 & & & 19.0 \\
\hline 115.00 & 3.20 & -63.6 & $\mathrm{pbd}+03$ & 0.20 & 2.70 & $\mathrm{pbd}+03$ & & & & kout & hh09 & 6.7 & 12.9 & 28.1 & 5.9 & 11.7 & 25.8 & 5.2 & 11.7 & 23.7 \\
\hline 115.80 & -1.60 & -40.8 & $\mathrm{pbd}+03$ & 1.60 & 2.70 & $\mathrm{pbd}+03$ & 2.5 & 0.5 & rus 03 & & & & & 29.1 & & & 29.1 & & & 29.1 \\
\hline 117.60 & 2.30 & -43.7 & rus03 & 2.30 & 2.70 & $\mathrm{pbd}+03$ & 2.6 & 0.27 & rag07 & & & & & 33.8 & & & 33.8 & & & 33.8 \\
\hline 118.10 & 5.00 & -9.1 & $\mathrm{pbd}+03$ & 9.50 & 2.70 & $\mathrm{pbd}+03$ & 0.7 & 0.04 & rus 03 & & & & & 22.6 & & & 22.6 & & & 22.6 \\
\hline 118.60 & -1.30 & -40.8 & $\mathrm{pbd}+03$ & 2.10 & 2.70 & $\mathrm{pbd}+03$ & 3.12 & 0.34 & rag07 & & & & & 36.8 & & & 36.8 & & & 36.8 \\
\hline 119.40 & -0.90 & & $\mathrm{pbd}+03$ & 2.60 & 2.70 & $\mathrm{pbd}+03$ & 3.12 & 0.34 & rag07 & & & & & 39.5 & & & 39.5 & & & 39.5 \\
\hline 119.40 & -0.80 & -34.5 & $\mathrm{pbd}+03$ & 0.60 & 2.70 & $\mathrm{pbd}+03$ & 3.12 & 0.34 & rag07 & & & & & 24.2 & & & 24.2 & & & 24.2 \\
\hline 120.40 & 2.00 & -49.0 & rus03 & 0.10 & 2.70 & $\mathrm{pbd}+03$ & 1.09 & 0.21 & rag07 & & & & & 6.3 & & & 6.3 & & & 6.3 \\
\hline 122.60 & 0.10 & -42.3 & rus03 & & & & 5.3 & 1.6 & rus03 & & & & & & & & & & & \\
\hline 123.00 & 3.00 & -10.3 & $\mathrm{pbd}+03$ & 0.30 & 2.70 & $\mathrm{pbd}+03$ & & & & kout & hh09 & 1.1 & 9.2 & 9.6 & 0.8 & 8.5 & 7.8 & 0.5 & 8.8 & 5.7 \\
\hline 123.10 & -6.30 & -29.7 & $\mathrm{pbd}+03$ & 8.40 & 2.70 & $\mathrm{pbd}+03$ & 2.9 & 0.4 & rus03 & & & & & 55.9 & & & 55.9 & & & 55.9 \\
\hline 123.20 & -6.30 & -30.4 & $\mathrm{pbd}+03$ & 4.30 & 2.70 & $\mathrm{pbd}+03$ & 2.9 & 0.4 & rus03 & & & & & 44.7 & & & 44.7 & & & 44.7 \\
\hline 124.00 & -1.90 & +3.0 & rus03 & & & & 0.2 & 0.1 & rus03 & & & & & & & & & & & \\
\hline 124.90 & 0.30 & -43.0 & $\mathrm{pbd}+03$ & 0.20 & 2.70 & $\mathrm{pbd}+03$ & & & & kout & hh09 & 4.2 & 11.5 & 20.6 & 3.6 & 10.5 & 18.6 & 3.1 & 10.6 & 16.8 \\
\hline 132.20 & -0.70 & -56.6 & $\mathrm{pbd}+03$ & 0.60 & 2.70 & $\mathrm{pbd}+03$ & & & & kout & hh09 & 6.2 & 13.5 & 38.5 & 5.3 & 12.3 & 34.7 & 4.6 & 12.1 & 31.6 \\
\hline 133.70 & 1.20 & -40.1 & $\mathrm{pbd}+03$ & 79.10 & 2.70 & $\mathrm{pbd}+03$ & 2.3 & 0.25 & rus 03 & & & & & 101.1 & & & 101.1 & & & 101.1 \\
\hline 133.80 & 1.40 & -47.7 & $\mathrm{pbd}+03$ & 17.90 & 2.70 & $\mathrm{pbd}+03$ & 2.3 & 0.25 & rus03 & & & & & 61.6 & & & 61.6 & & & 61.6 \\
\hline 136.10 & 2.10 & -46.3 & rus03 & 0.10 & 2.70 & $\mathrm{pbd}+03$ & 2.4 & 0.32 & rag07 & & & & & 11.3 & & & 11.3 & & & 11.3 \\
\hline 136.50 & 2.50 & -45.1 & $\mathrm{pbd}+03$ & 0.20 & 2.70 & $\mathrm{pbd}+03$ & & & & kout & hh09 & 4.9 & 12.5 & 22.8 & 4.2 & 11.4 & 20.6 & 3.6 & 11.4 & 18.6 \\
\hline 136.90 & 1.00 & -41.3 & $\mathrm{pbd}+03$ & 2.50 & 2.70 & $\mathrm{pbd}+03$ & 2.3 & 0.25 & rus 03 & & & & & 32.0 & & & 32.0 & & & 32.0 \\
\hline 138.50 & 1.60 & -34.6 & $\mathrm{pbd}+03$ & 1.20 & 2.70 & $\mathrm{pbd}+03$ & 2.3 & 0.25 & rus 03 & & & & & 25.0 & & & 25.0 & & & 25.0 \\
\hline 140.00 & 2.00 & -10.7 & rus03 & & & & 1.1 & 0.2 & rus03 & & & & & & & & & & & \\
\hline 143.60 & -1.80 & -34.4 & rus03 & 12.00 & 2.70 & $\mathrm{pbd}+03$ & 1.71 & 0.31 & rag07 & & & & & 44.1 & & & 44.1 & & & 44.1 \\
\hline 145.80 & 3.00 & -26.0 & rus03 & & & & 4.0 & 1.2 & rus 03 & & & & & & & & & & & \\
\hline 148.50 & -0.20 & -17.5 & rus03 & & & & 0.8 & 0.2 & rus03 & & & & & 20.0 & & & 20.0 & & & 20.0 \\
\hline 150.60 & 0.90 & -25.1 & $\mathrm{pbd}+03$ & 17.30 & 2.70 & $\mathrm{pbd}+03$ & 2.7 & 0.8 & rus03 & & & & & 67.8 & & & 67.8 & & & 67.8 \\
\hline 151.30 & 2.00 & -32.9 & rus03 & 0.10 & 2.70 & $\mathrm{pbd}+03$ & 7.5 & 1.6 & rus03 & & & & & 24.0 & & & 24.0 & & & 24.0 \\
\hline 151.60 & -0.20 & -49.9 & $\mathrm{pbd}+03$ & 12.40 & 2.70 & $\mathrm{pbd}+03$ & 10.1 & 1.7 & rus 03 & & & & & 145.3 & & & 145. & & & 145. \\
\hline 154.60 & 2.40 & -35.5 & $\mathrm{pbd}+03$ & 0.70 & 2.70 & $\mathrm{pbd}+03$ & 6.8 & 0.9 & rus 03 & & & & & 43.1 & & & 43.1 & & & 43.1 \\
\hline 155.40 & 2.60 & -40.1 & $\mathrm{pbd}+03$ & 1.60 & 2.70 & $\mathrm{pbd}+03$ & 6.8 & 0.9 & rus 03 & & & & & 56.8 & & & 56.8 & & & 56.8 \\
\hline 157.10 & -3.60 & -31.0 & $\mathrm{pbd}+03$ & 0.10 & 2.70 & $\mathrm{pbd}+03$ & & & & kout & hh09 & 5.6 & 13.8 & 19.8 & 4.8 & 12.5 & 17.9 & 3.9 & 12.2 & 15.6 \\
\hline 159.10 & 3.30 & -20.5 & $\mathrm{pbd}+03$ & 0.40 & 2.70 & $\mathrm{pbd}+03$ & 4.19 & 0.27 & rag07 & & & & & 25.5 & & & 25.5 & & & 25.5 \\
\hline 159.40 & 2.60 & -24.5 & $\mathrm{pbd}+03$ & 0.10 & 2.70 & $\mathrm{pbd}+03$ & 4.19 & 0.27 & rag07 & & & & & 16.1 & & & 16.1 & & & 16.1 \\
\hline 160.30 & 0.90 & -25.2 & $\mathrm{pbd}+03$ & 0.10 & 2.70 & $\mathrm{pbd}+03$ & 4.19 & 0.27 & rag07 & & & & & 16.1 & & & 16.1 & & & 16.1 \\
\hline 161.00 & 12.00 & 7.0 & rus03 & & & & 0.5 & 0.2 & rus03 & & & & & 37.0 & & & 37.0 & & & 37.0 \\
\hline 168.10 & 3.10 & -22.6 & rus03 & 0.10 & 2.70 & $\mathrm{pbd}+03$ & 3.8 & 1.1 & rus03 & & & & & 15.3 & & & 15.3 & & & 15.3 \\
\hline 168.60 & 0.00 & -27.1 & rus03 & 0.60 & 2.70 & $\mathrm{pbd}+03$ & 4.2 & 1.3 & rus 03 & & & & & 29.7 & & & 29.7 & & & 29.7 \\
\hline 169.20 & -0.90 & -20.5 & $\mathrm{pbd}+03$ & 1.50 & 2.70 & $\mathrm{pbd}+03$ & 4.1 & 1.2 & rus 03 & & & & & 39.7 & & & 39.7 & & & 39.7 \\
\hline 172.50 & 2.00 & & rus03 & & & & 0.5 & 0.1 & rus 03 & & & & & 23.0 & & & 23.0 & & & 23.0 \\
\hline 173.40 & -0.20 & -14.3 & rus03 & 1.50 & 2.70 & $\mathrm{pbd}+03$ & 1.8 & 0.25 & rus 03 & & & & & 22.9 & & & 22.9 & & & 22.9 \\
\hline 173.60 & 2.80 & -24.5 & $\mathrm{pbd}+03$ & 3.70 & 2.70 & $\mathrm{pbd}+03$ & 1.6 & 0.3 & rus03 & & & & & 28.6 & & & 28.6 & & & 28.6 \\
\hline 173.60 & -1.80 & -7.2 & $\mathrm{pbd}+03$ & 2.10 & 2.70 & $\mathrm{pbd}+03$ & 4.3 & 0.25 & rus03 & & & & & 45.8 & & & 45.8 & & & 45.8 \\
\hline 173.90 & 0.30 & -4.3 & rus03 & 0.80 & 2.70 & $\mathrm{pbd}+03$ & 5.2 & 0.8 & rus 03 & & & & & 37.7 & & & 37.7 & & & 37.7 \\
\hline 180.90 & 4.10 & -6.5 & rus03 & 1.40 & 2.70 & $\mathrm{pbd}+03$ & 4.9 & 1.0 & rus 03 & & & & & 43.6 & & & 43.6 & & & 43.6 \\
\hline 182.40 & 0.20 & 0.0 & rus03 & 0.90 & 2.70 & $\mathrm{pbd}+03$ & 2.2 & 0.7 & rus03 & & & & & 22.1 & & & 22.1 & & & 22.1 \\
\hline 188.90 & 0.80 & 3.0 & rus03 & 0.90 & 2.70 & $\mathrm{pbd}+03$ & 4.2 & 1.3 & rus 03 & & & & & 34.0 & & & 34.0 & & & 34.0 \\
\hline 189.00 & 4.00 & -5.4 & rus03 & & & & 1.8 & 0.3 & rus 03 & & & & & 56.0 & & & 56.0 & & & 56.0 \\
\hline 189.80 & 0.30 & 7.5 & $\mathrm{pbd}+03$ & 0.20 & 2.70 & $\mathrm{pbd}+03$ & 2.1 & 0.03 & $\mathrm{rmb}+08$ & & & & & 13.0 & & & 13.0 & & & 13.0 \\
\hline 192.20 & 3.60 & 14.4 & rus03 & 0.10 & 2.70 & $\mathrm{pbd}+03$ & 5.1 & 1.5 & rus03 & & & & & 18.6 & & & 18.6 & & & 18.6 \\
\hline 192.60 & -0.00 & 5.8 & $\mathrm{pbd}+03$ & 1.40 & 2.70 & $\mathrm{pbd}+03$ & 2.46 & 0.16 & rag07 & & & & & 27.1 & & & 27.1 & & & 27.1 \\
\hline 192.90 & -0.60 & 22.8 & rus03 & 0.30 & 2.70 & $\mathrm{pbd}+03$ & 8.9 & 2.7 & rus03 & & & & & 38.9 & & & 38.9 & & & 38.9 \\
\hline
\end{tabular}


Table A.1. continued.

\begin{tabular}{|c|c|c|c|c|c|c|c|c|c|c|c|c|c|c|c|c|c|c|c|c|}
\hline $\begin{array}{r}l \\
\left({ }^{\circ}\right) \\
(1)\end{array}$ & $\begin{array}{r}b \\
\left({ }^{\circ}\right) \\
(2)\end{array}$ & $\begin{array}{c}V_{\mathrm{lsr}} \\
\mathrm{km} \mathrm{s}^{-1} \\
(3)\end{array}$ & Ref. & $\begin{array}{c}S \\
\text { Jy } \\
(5)\end{array}$ & $\begin{array}{r}\text { Freq } \\
\text { GHz } \\
(6)\end{array}$ & $\begin{array}{l}\text { Ref. } \\
\text { (7) }\end{array}$ & $\begin{array}{r}d \\
\mathrm{kpc} \\
(8)\end{array}$ & $\begin{array}{r}d_{\text {erro }} \\
\text { kpc } \\
(9)\end{array}$ & Ref. & Mark & $\begin{array}{l}\text { Ref. } \\
\text { (12) }\end{array}$ & $\begin{array}{l}D_{8.5} \\
\mathrm{kpc} \\
(13)\end{array}$ & $\begin{array}{l}R_{8.5} \\
\mathrm{kpc} \\
(14)\end{array}$ & $\begin{array}{r}U_{8.5} \\
\mathrm{pc} \mathrm{cm} \\
(15)\end{array}$ & $\begin{array}{l}D_{8.0} \\
\text { kpc } \\
(16)\end{array}$ & $\begin{array}{l}R_{8.0} \\
\mathrm{kpc} \\
(17)\end{array}$ & $\begin{array}{r}U_{8.0} \\
\mathrm{pc} \mathrm{cm}^{-2} \\
(18)\end{array}$ & $\begin{array}{l}D_{8.4} \\
\text { kpc } \\
(19)\end{array}$ & $\begin{array}{l}R_{8.4} \\
\mathrm{kpc} \\
(20)\end{array}$ & $\begin{array}{r}U_{8.4} \\
\mathrm{pc} \mathrm{cm} \\
(21)\end{array}$ \\
\hline 193.70 & -2.20 & 6.0 & $\begin{array}{ll}\text { rus03 } \\
\end{array}$ & & & & 1.6 & 0.5 & rus03 & & & & & & & & & & & \\
\hline 194.70 & 15.60 & 0.3 & rus03 & & & & 0.5 & 0.1 & rus 03 & & & & & 7.0 & & & 7.0 & & & 7.0 \\
\hline 195.10 & 12.00 & 12.0 & rus03 & & & & 0.5 & 0.1 & rus 03 & & & & & & & & & & & \\
\hline 195.70 & -0.10 & 28.6 & rus03 & 0.20 & 2.70 & $\mathrm{pbd}+03$ & 2.0 & 0.6 & rus03 & & & & & 12.6 & & & 12.6 & & & 12.6 \\
\hline 196.40 & -1.70 & 17.1 & $\mathrm{pbd}+03$ & 0.70 & 2.70 & $\mathrm{pbd}+03$ & 4.2 & 1.3 & rus03 & & & & & 31.3 & & & 31.3 & & & 31.3 \\
\hline 196.40 & -2.90 & 6.3 & rus03 & & & & 1.3 & 0.4 & rus03 & & & & & 42.0 & & & 42.0 & & & 42.0 \\
\hline 196.80 & -3.10 & 25.6 & $\mathrm{pbd}+03$ & 0.10 & 2.70 & $\mathrm{pbd}+03$ & & & & kout & hh09 & 6.4 & 14.8 & 21.6 & 5.5 & 13.4 & 19.5 & 4.8 & 13.2 & 17.8 \\
\hline 196.90 & 10.40 & 12.0 & $\mathrm{pbd}+03$ & 0.10 & 2.70 & $\mathrm{pbd}+03$ & & & & kout & hh09 & 2.3 & 10.8 & 10.9 & 2.0 & 9.9 & 9.9 & 1.9 & 10.3 & 9.6 \\
\hline 197.80 & -2.30 & 19.2 & rus03 & 0.30 & 2.70 & $\mathrm{pbd}+03$ & 5.1 & 1.1 & rus03 & & & & & 26.8 & & & 26.8 & & & 26.8 \\
\hline 202.90 & 2.20 & 7.0 & rus 03 & & & & 0.8 & 0.04 & rus 03 & & & & & & & & & & & \\
\hline 206.30 & -2.10 & 14.8 & rus03 & & & & 1.9 & 0.25 & rus 03 & & & & & 126.0 & & & 126.0 & & & 126.0 \\
\hline 206.50 & 16.40 & 5.0 & $\mathrm{pbd}+03$ & 77.20 & 2.70 & $\mathrm{pbd}+03$ & 0.4 & 0.04 & rus 03 & & & & & 31.3 & & & 31.3 & & & 31.3 \\
\hline 206.60 & 16.20 & 9.6 & $\mathrm{pbd}+03$ & 0.10 & 2.70 & $\mathrm{pbd}+03$ & 0.4 & 0.04 & rus 03 & & & & & 3.4 & & & 3.4 & & & 3.4 \\
\hline 208.40 & 19.10 & 8.0 & $\mathrm{pbd}+03$ & 2.30 & 2.70 & $\mathrm{pbd}+03$ & 0.4 & 0.04 & rus 03 & & & & & 9.7 & & & 9.7 & & & 9.7 \\
\hline 208.70 & -2.60 & 11.1 & rus03 & 1.80 & 2.70 & $\mathrm{pbd}+03$ & 1.4 & 0.4 & rus 03 & & & & & 20.6 & & & 20.6 & & & 20.6 \\
\hline 208.90 & 19.30 & 7.7 & $\mathrm{pbd}+03$ & 23.00 & 2.70 & $\mathrm{pbd}+03$ & 0.4 & 0.04 & rus 03 & & & & & 20.9 & & & 20.9 & & & 20.9 \\
\hline 209.00 & 19.40 & -2.3 & $\mathrm{pbd}+03$ & 392.00 & 2.70 & $\mathrm{pbd}+03$ & 0.4 & 0.04 & rus 03 & & & & & 53.7 & & & 53.7 & & & 53.7 \\
\hline 210.60 & -1.40 & 22.0 & $\operatorname{rag} 07$ & 0.10 & 2.70 & $\mathrm{pbd}+03$ & 1.17 & 0.29 & rag07 & & & & & 6.7 & & & 6.7 & & & 6.7 \\
\hline 210.80 & -2.60 & 49.4 & $\mathrm{pbd}+03$ & 0.10 & 2.70 & $\mathrm{pbd}+03$ & 7.89 & 0.27 & rag07 & & & & & 24.7 & & & 24.7 & & & 24.7 \\
\hline 211.14 & -1.01 & 37.1 & rag07 & & & & 4.01 & 0.73 & rag07 & & & & & & & & & & & \\
\hline 211.90 & -1.20 & 45.0 & $\mathrm{pbd}+03$ & 0.10 & 2.70 & $\mathrm{pbd}+03$ & 7.89 & 0.27 & rag07 & & & & & 24.7 & & & 24.7 & & & 24.7 \\
\hline 213.70 & 12.60 & 12.0 & $\mathrm{pbd}+03$ & 9.80 & 2.70 & $\mathrm{pbd}+03$ & & & & kout & hh09 & 1.3 & 9.6 & 34.5 & 1.1 & 8.9 & 30.9 & 0.9 & 9.3 & 27.0 \\
\hline 213.80 & 0.60 & 45.3 & $\mathrm{pbd}+03$ & 0.10 & 2.70 & $\mathrm{pbd}+03$ & 7.89 & 0.27 & rag07 & & & & & 24.7 & & & 24.7 & & & 24.7 \\
\hline 216.10 & 0.00 & 22.6 & rus03 & 0.10 & 2.70 & $\mathrm{pbd}+03$ & 1.7 & 0.5 & rus03 & & & & & 8.9 & & & 8.9 & & & 8.9 \\
\hline 218.10 & -0.30 & 27.2 & $\mathrm{pbd}+03$ & 0.30 & 2.70 & $\mathrm{pbd}+03$ & 2.9 & 0.5 & rus03 & & & & & 18.4 & & & 18.4 & & & 18.4 \\
\hline 218.70 & 1.80 & 55.0 & rus03 & & & & 7.5 & 2.2 & rus 03 & & & & & 43.0 & & & 43.0 & & & 43.0 \\
\hline 218.80 & -4.60 & 51.1 & rus03 & 0.30 & 2.70 & $\mathrm{pbd}+03$ & 8.5 & 0.25 & rus03 & & & & & 37.7 & & & 37.7 & & & 37.7 \\
\hline 220.70 & -2.20 & 9.9 & $\mathrm{pbd}+03$ & 0.10 & 2.70 & $\mathrm{pbd}+03$ & & & & kout & hh09 & 1.0 & 9.3 & 6.3 & 0.8 & 8.6 & 5.4 & 0.5 & 8.9 & 4.0 \\
\hline 220.90 & -2.50 & 13.4 & $\mathrm{pbd}+03$ & 0.30 & 2.70 & $\mathrm{pbd}+03$ & & & & kout & hh09 & 1.3 & 9.6 & 10.8 & 1.0 & 8.8 & 9.1 & 0.8 & 9.1 & 7.8 \\
\hline 221.80 & -2.00 & 40.0 & $\mathrm{pbd}+03$ & 0.10 & 2.70 & $\mathrm{pbd}+03$ & & & & kout & hh09 & 4.3 & 12.0 & 16.6 & 3.7 & 11.0 & 15.0 & 3.2 & 11.1 & 13.6 \\
\hline 224.10 & -2.00 & 18.4 & $\mathrm{pbd}+03$ & 3.60 & 2.70 & $\mathrm{pbd}+03$ & 1.0 & 0.04 & rus03 & & & & & 20.7 & & & 20.7 & & & 20.7 \\
\hline 224.20 & -2.90 & 14.6 & $\mathrm{pbd}+03$ & 0.10 & 2.70 & $\mathrm{pbd}+03$ & 1.0 & 0.04 & rus03 & & & & & 6.3 & & & 6.3 & & & 6.3 \\
\hline 224.20 & 1.20 & 33.6 & rus03 & 0.50 & 2.70 & $\mathrm{pbd}+03$ & 3.24 & 0.56 & rag07 & & & & & 23.3 & & & 23.3 & & & 23.3 \\
\hline 227.80 & -0.20 & 56.5 & rus 03 & & & & 3.7 & 1.1 & rus03 & & & & & 54.0 & & & 54.0 & & & 54.0 \\
\hline 231.50 & -4.30 & 53.0 & $\mathrm{pbd}+03$ & 3.90 & 2.70 & $\mathrm{pbd}+03$ & & & & kout & hh09 & 5.5 & 12.7 & 66.3 & 4.7 & 11.6 & 59.7 & 4.2 & 11.5 & 55.4 \\
\hline 232.60 & 0.90 & 17.8 & rus03 & & & & 2.5 & 0.7 & rus03 & & & & & 29.0 & & & 29.0 & & & 29.0 \\
\hline 233.80 & -0.20 & 40.2 & $\mathrm{pbd}+03$ & 1.70 & 2.70 & $\mathrm{pbd}+03$ & 4.3 & 0.4 & rus 03 & & & & & 42.7 & & & 42.7 & & & 42.7 \\
\hline 234.30 & -0.40 & 44.1 & $\mathrm{pbd}+03$ & 1.00 & 2.70 & $\mathrm{pbd}+03$ & & & & kout & hh09 & 4.4 & 11.6 & 36.3 & 3.7 & 10.6 & 32.3 & 3.2 & 10.7 & 29.4 \\
\hline 234.60 & -0.20 & 44.0 & $\mathrm{pbd}+03$ & 0.60 & 2.70 & $\mathrm{pbd}+03$ & & & & kout & hh09 & 4.3 & 11.6 & 30.2 & 3.7 & 10.6 & 27.3 & 3.2 & 10.7 & 24.8 \\
\hline 234.80 & 10.10 & 16.0 & rus03 & & & & 1.3 & 0.4 & rus03 & & & & & 17.0 & & & 17.0 & & & 17.0 \\
\hline 235.50 & -4.10 & 28.2 & rus 03 & & & & 2.5 & 0.4 & rus03 & & & & & & & & & & & \\
\hline 236.60 & -2.10 & 26.3 & rus03 & & & & 2.3 & 0.7 & rus 03 & & & & & & & & & & & \\
\hline 237.00 & -1.30 & 74.8 & rus03 & & & & 5.4 & 2.0 & rus 03 & & & & & & & & & & & \\
\hline 237.30 & -6.50 & 18.0 & rus03 & & & & 1.6 & 0.25 & rus 03 & & & & & & & & & & & \\
\hline 238.50 & -4.70 & 20.6 & rus03 & & & & 1.4 & 0.1 & rus 03 & & & & & & & & & & & \\
\hline 241.50 & -0.60 & 70.1 & rus 03 & & & & 4.7 & 0.6 & rus 03 & & & & & & & & & & & \\
\hline 243.20 & 0.40 & 53.9 & $\mathrm{pbd}+03$ & 14.70 & 2.70 & $\mathrm{pbd}+03$ & 4.0 & 0.25 & rus 03 & & & & & 83.5 & & & 83.5 & & & 83.5 \\
\hline 246.00 & 1.20 & 62.9 & rus03 & & & & 6.7 & 1.1 & rus 03 & & & & & & & & & & & \\
\hline 247.50 & 2.20 & 40.8 & rus 03 & & & & 4.0 & 0.8 & rus 03 & & & & & & & & & & & \\
\hline 247.80 & -0.50 & 55.2 & rus03 & & & & 3.2 & 0.4 & rus 03 & & & & & & & & & & & \\
\hline 248.00 & -5.50 & 51.3 & rus03 & & & & 4.4 & 0.5 & rus 03 & & & & & & & & & & & \\
\hline 253.00 & -1.30 & 9.5 & rus03 & & & & 1.3 & 0.3 & rus03 & & & & & & & & & & & \\
\hline 253.60 & -0.20 & 36.3 & $\mathrm{pbd}+03$ & 19.00 & 2.70 & $\mathrm{pbd}+03$ & 3.0 & 0.3 & rus03 & & & & & 75.1 & & & 75.1 & & & 75.1 \\
\hline 254.70 & 0.20 & 64.0 & $\mathrm{pbd}+03$ & 3.80 & 2.70 & $\mathrm{pbd}+03$ & 7.6 & 0.9 & cor+07 & & & & & 81.6 & & & 81.6 & & & 81.6 \\
\hline 255.70 & -2.40 & 9.6 & rus03 & & & & 0.5 & 0.1 & rus03 & & & & & & & & & & & \\
\hline 260.10 & 0.60 & 7.1 & rus03 & & & & 1.2 & 0.2 & rus 03 & & & & & & & & & & & \\
\hline 261.40 & 0.80 & 7.1 & rus03 & & & & 1.0 & 0.04 & rus 03 & & & & & & & & & & & \\
\hline 262.90 & -2.40 & 8.7 & rus03 & & & & 0.6 & 0.03 & rus03 & & & & & & & & & & & \\
\hline 263.00 & 1.50 & 5.5 & rus03 & & & & 1.1 & 0.2 & rus03 & & & & & & & & & & & \\
\hline 263.60 & -0.50 & 1.0 & $\mathrm{pbd}+03$ & 2.10 & 2.70 & $\mathrm{pbd}+03$ & & & & kout & hh09 & 1.0 & 8.7 & 17.3 & 0.3 & 8.0 & 7.8 & & & \\
\hline 264.30 & 1.50 & 12.0 & $\mathrm{pbd}+03$ & 3.20 & 2.70 & $\mathrm{pbd}+03$ & 3.1 & 0.9 & rus03 & & & & & 42.4 & & & 42.4 & & & 42.4 \\
\hline 264.30 & 0.30 & 9.0 & rus03 & & & & 1.7 & 0.3 & rus03 & & & & & & & & & & & \\
\hline 264.40 & 1.10 & 6.0 & rus 03 & & & & 0.9 & 0.1 & rus03 & & & & & & & & & & & \\
\hline 265.10 & 1.50 & 2.3 & $\mathrm{pbd}+03$ & 31.70 & 2.70 & $\mathrm{pbd}+03$ & & & & kout & hh09 & 1.4 & 8.7 & 53.6 & 0.7 & 8.1 & 33.8 & & & \\
\hline 265.20 & 1.50 & 3.0 & $\mathrm{pbd}+03$ & 25.10 & 2.70 & $\mathrm{pbd}+03$ & & & & kout & hh09 & 1.5 & 8.8 & 51.9 & 0.8 & 8.1 & 34.1 & & & \\
\hline
\end{tabular}


Table A.1. continued.

\begin{tabular}{|c|c|c|c|c|c|c|c|c|c|c|c|c|c|c|c|c|c|c|c|c|}
\hline $\begin{array}{r}l \\
\left({ }^{\circ}\right) \\
(1)\end{array}$ & $\begin{array}{r}b \\
\left({ }^{\circ}\right) \\
(2)\end{array}$ & $\begin{array}{c}V_{\mathrm{lsr}} \\
\mathrm{km} \mathrm{s}^{-1} \\
(3)\end{array}$ & Ref. & $\begin{array}{c}S \\
\text { Jy } \\
(5)\end{array}$ & $\begin{array}{r}\text { Freq } \\
\text { GHz } \\
(6)\end{array}$ & Ref. & $\begin{array}{r}d \\
\mathrm{kpc} \\
(8)\end{array}$ & $\begin{array}{r}d_{\text {erro }} \\
\text { kpc } \\
(9)\end{array}$ & (10) & Mark & (12) & $\begin{array}{l}D_{8.5} \\
\mathrm{kpc} \\
(13)\end{array}$ & $\begin{array}{c}R_{8.5} \\
\mathrm{kpc} \\
(14)\end{array}$ & $\begin{array}{r}U_{8.5} \\
\mathrm{pc} \mathrm{cm}^{-2} \\
(15)\end{array}$ & $\begin{array}{c}D_{8.0} \\
\mathrm{kpc} \\
(16)\end{array}$ & $\begin{array}{l}R_{8.0} \\
\mathrm{kpc} \\
(17)\end{array}$ & $\begin{array}{r}U_{8.0} \\
\mathrm{pc} \mathrm{cm}^{-2} \\
(18)\end{array}$ & $\begin{array}{r}D_{8.4} \\
\mathrm{kpc} \\
(19)\end{array}$ & $\begin{array}{l}R_{8.4} \\
\mathrm{kpc} \\
(20)\end{array}$ & $\begin{array}{r}U_{8.4} \\
\mathrm{pc} \mathrm{cm}^{-2} \\
(21)\end{array}$ \\
\hline 267.80 & -0.90 & 7.9 & $\mathrm{pbd}+03$ & 21.60 & 2.70 & $\mathrm{pbd}+03$ & 1.6 & 0.2 & rus03 & & & & & 51.5 & & & 51.5 & & & 51.5 \\
\hline 267.90 & -1.10 & 1.0 & $\mathrm{pbd}+03$ & 153.30 & 2.70 & $\mathrm{pbd}+03$ & 1.6 & 0.2 & rus03 & & & & & 99.0 & & & 99.0 & & & 99.0 \\
\hline 268.00 & -1.10 & 1.8 & $\mathrm{pbd}+03$ & 183.30 & 2.70 & $\mathrm{pbd}+03$ & & & & kout & hh09 & 1.6 & 8.7 & 105.1 & 0.8 & 8.1 & 66.2 & & & \\
\hline 268.00 & -1.00 & 1.8 & $\mathrm{pbd}+03$ & 19.40 & 2.70 & $\mathrm{pbd}+03$ & & & & kout & hh09 & 1.6 & 8.7 & 49.7 & 0.8 & 8.1 & 31.3 & & & \\
\hline 268.00 & 1.80 & -0.9 & rus03 & & & & 0.5 & 0.1 & rus03 & & & & & & & & & & & \\
\hline 268.20 & -2.70 & 13.0 & rus03 & & & & 2.6 & 0.5 & rus03 & & & & & & & & & & & \\
\hline 268.50 & -0.80 & 5.0 & $\mathrm{pbd}+03$ & 6.60 & 2.70 & $\mathrm{pbd}+03$ & 1.3 & 0.4 & rus 03 & & & & & 30.2 & & & 30.2 & & & 30.2 \\
\hline 269.10 & -1.10 & 15.0 & $\mathrm{pbd}+03$ & 8.40 & 2.70 & $\mathrm{pbd}+03$ & 2.6 & 0.8 & rus 03 & & & & & 52.0 & & & 52.0 & & & 52.0 \\
\hline 269.50 & -1.50 & 71.0 & $\mathrm{pbd}+03$ & 1.30 & 2.70 & $\mathrm{pbd}+03$ & & & & kout & hh09 & 9.7 & 12.9 & 67.1 & 8.6 & 11.8 & 61.9 & 8.0 & 11.7 & 59.0 \\
\hline 270.30 & 0.80 & 1.0 & $\mathrm{pbd}+03$ & 4.90 & 2.70 & $\mathrm{pbd}+03$ & 3.4 & 0.7 & rus03 & & & & & 51.9 & & & 51.9 & & & 51.9 \\
\hline 270.80 & 0.70 & 5.4 & rus03 & & & & 1.4 & 0.3 & rus03 & & & & & & & & & & & \\
\hline 274.00 & -1.10 & 39.0 & $\mathrm{pbd}+03$ & 32.30 & 2.70 & $\mathrm{pbd}+03$ & 2.7 & 0.8 & rus 03 & & & & & 83.5 & & & 83.5 & & & 83.5 \\
\hline 274.70 & -1.50 & 3.1 & rus03 & & & & 4.4 & 0.9 & rus03 & & & & & & & & & & & \\
\hline 281.60 & -1.00 & 2.0 & $\mathrm{pbd}+03$ & 1.10 & 2.70 & $\mathrm{pbd}+03$ & & & & kout & hh09 & 4.3 & 8.7 & 36.9 & 3.6 & 8.1 & 32.8 & 2.8 & 8.4 & 27.7 \\
\hline 281.80 & -2.00 & -5.4 & rus03 & & & & 4.7 & 1.0 & rus03 & & & & & & & & & & & \\
\hline 282.00 & -1.20 & 21.0 & $\mathrm{pbd}+03$ & 36.20 & 2.70 & $\mathrm{pbd}+03$ & & & & kout & hh09 & 6.6 & 9.6 & 157.4 & 5.8 & 8.9 & 144.4 & 5.5 & 9.1 & 139.4 \\
\hline 282.20 & -1.10 & -2.0 & $\mathrm{pbd}+03$ & 11.40 & 2.70 & $\mathrm{pbd}+03$ & & & & kout & hh09 & 3.8 & 8.5 & 74.1 & & & & 1.8 & 8.3 & 45.0 \\
\hline 282.30 & -1.80 & -15.0 & $\mathrm{pbd}+03$ & 4.30 & 2.70 & $\mathrm{pbd}+03$ & 3.1 & 0.5 & rus03 & & & & & 46.8 & & & 46.8 & & & 46.8 \\
\hline 282.50 & -1.20 & -12.0 & rus03 & & & & 2.0 & 0.4 & rus03 & & & & & 55.0 & & & 55.0 & & & 55.0 \\
\hline 282.70 & -1.40 & -19.1 & rus03 & & & & 2.0 & 0.4 & rus03 & & & & & & & & & & & \\
\hline 283.10 & -1.00 & -1.0 & $\mathrm{pbd}+03$ & 5.10 & 2.70 & $\mathrm{pbd}+03$ & & & & kout & hh09 & 4.2 & 8.6 & 60.6 & & & & 2.4 & 8.3 & 41.7 \\
\hline 283.30 & -1.00 & 16.0 & $d+03$ & 6.10 & 2.70 & $\mathrm{pbd}+03$ & & & & kout & hh09 & 6.3 & 9.4 & 84.3 & 5.6 & 8.6 & 77.9 & 5.3 & 8.9 & 75.1 \\
\hline 283.30 & -0.60 & 6.0 & $d+03$ & 3.10 & 2.70 & $\mathrm{pbd}+03$ & & & & kout & hh09 & 5.2 & 8.9 & 59.2 & 4.5 & 8.2 & 53.8 & 4.1 & 8.5 & 50.5 \\
\hline 283.50 & -1.00 & -3.0 & $d+03$ & 8.90 & 2.70 & $\mathrm{pbd}+03$ & 3.3 & 0.7 & rus 03 & & & & & 62.1 & & & 62.1 & & & 62.1 \\
\hline 34.00 & -0.90 & 3.8 & $d+03$ & 16.80 & 2.70 & $\mathrm{pbd}+03$ & 5.5 & 0.25 & rus03 & & & & & 107.9 & & & 107.9 & & & 107.9 \\
\hline 284.10 & -0.40 & -16.0 & $d+03$ & 8.50 & 2.70 & $\mathrm{pbd}+03$ & & & & ktan & hh09 & 2.1 & 8.2 & 45.3 & 1.9 & 7.8 & 42.4 & 2.1 & 8.2 & 45.3 \\
\hline 284.30 & 0.40 & 1.0 & $d+03$ & 3.40 & 2.70 & $\mathrm{pbd}+03$ & 5.5 & 0.25 & rus03 & & & & & 63.4 & & & 63.4 & & & 63.4 \\
\hline 284.30 & -0.30 & -0.5 & $d+03$ & 41.40 & 2.70 & $\mathrm{pbd}+03$ & 5.5 & 0.25 & rus03 & & & & & 262.4 & & & 262.4 & & & 262.4 \\
\hline 284.60 & -0.50 & 5.0 & $d+03$ & 1.30 & 2.70 & $\mathrm{pbd}+03$ & & & & kout & hh09 & 5.4 & 8.8 & 45.4 & 4.7 & 8.2 & 41.4 & 4.3 & 8.5 & 39.0 \\
\hline 284.60 & -0.20 & 9.0 & $d+03$ & 10.30 & 2.70 & $\mathrm{pbd}+03$ & & & & kout & hh09 & 5.9 & 9.0 & 96.1 & 5.2 & 8.4 & 88.3 & 4.8 & 8.6 & 83.7 \\
\hline 284.70 & 0.30 & 10.0 & $d+03$ & 3.70 & 2.70 & $\mathrm{pbd}+03$ & & & & kout & hh09 & 6.0 & 9.1 & 69.1 & 5.3 & 8.4 & 63.6 & 4.9 & 8.7 & 60.4 \\
\hline 285.30 & -0.10 & -2.0 & $d+03$ & 13.30 & 2.70 & $\mathrm{pbd}+03$ & & & & kout & hh09 & 4.7 & 8.5 & 89.9 & & & & 3.2 & 8.3 & 69.6 \\
\hline 285.30 & -0.00 & -1.6 & $\mathrm{pbd}+03$ & 20.90 & 2.70 & $\mathrm{pbd}+03$ & & & & kout & hh09 & 4.7 & 8.6 & 104.5 & & & & 3.3 & 8.3 & 82.6 \\
\hline 286.20 & -0.20 & -18.0 & $\mathrm{pbd}+03$ & 37.20 & 2.70 & $\mathrm{pbd}+03$ & 2.6 & 0.25 & rus03 & & & & & 85.4 & & & 85.4 & & & 85.4 \\
\hline 286.40 & -1.50 & 38.0 & $\mathrm{pbd}+03$ & 2.20 & 2.70 & $\mathrm{pbd}+03$ & & & & kout & hh09 & 9.2 & 10.6 & 77.2 & 8.3 & 9.8 & 72.1 & 8.0 & 9.9 & 70.3 \\
\hline 286.40 & -1.30 & 38.0 & $\mathrm{pbd}+03$ & 3.20 & 2.70 & $\mathrm{pbd}+03$ & & & & kout & hh09 & 9.2 & 10.6 & 87.5 & 8.3 & 9.8 & 81.7 & 8.0 & 9.9 & 79.7 \\
\hline 286.70 & 3.00 & -13.0 & rus03 & & & & 2.1 & 0.6 & rus03 & & & & & & & & & & & \\
\hline 286.90 & -0.90 & -20.0 & $\mathrm{pbd}+03$ & 15.00 & 2.70 & $\mathrm{pbd}+03$ & 2.6 & 0.25 & rus03 & & & & & 63.1 & & & 63.1 & & & 63.1 \\
\hline 287.20 & -0.70 & -22.4 & $d+03$ & 15.10 & 2.70 & $\mathrm{pbd}+03$ & & & & $\mathrm{ktan}$ & hh09 & 2.5 & 8.1 & 61.6 & 2.4 & 7.6 & 59.9 & 2.5 & 8.0 & 61.6 \\
\hline 287.20 & 0.10 & -25.0 & $d+03$ & 6.70 & 2.70 & $\mathrm{pbd}+03$ & 2.6 & 0.25 & rus03 & & & & & 48.2 & & & 48.2 & & & 48.2 \\
\hline 287.20 & 0.40 & 18.0 & $d+03$ & 3.50 & 2.70 & $\mathrm{pbd}+03$ & 2.6 & 0.25 & rus03 & & & & & 38.8 & & & 38.8 & & & 38.8 \\
\hline 287.30 & -0.90 & -17.0 & $d+03$ & 16.10 & 2.70 & $\mathrm{pbd}+03$ & & & & ktan & hh09 & 2.5 & 8.1 & 62.9 & 2.4 & 7.6 & 61.2 & 2.5 & 8.0 & 62.9 \\
\hline 287.40 & .60 & -17.7 & $d+03$ & 147.60 & 2.70 & $\mathrm{pbd}+03$ & 2.6 & 0.25 & rus03 & & & & & 35.1 & & & 135.1 & & & 135.1 \\
\hline 7.50 & .60 & 19.6 & & & 2.70 & & 2.6 & 0.25 & rus & & & & & 116.2 & & & 116.2 & & & 116.2 \\
\hline 287.60 & -0.90 & -24.0 & $d+03$ & 29.80 & 2.70 & $\mathrm{pbd}+03$ & 2.6 & 0.25 & rus 03 & & & & & 79.3 & & & 79.3 & & & 79.3 \\
\hline 287.70 & -0.60 & -26.0 & $d+03$ & 11.10 & 2.70 & $\mathrm{pbd}+03$ & & & & $\mathrm{ktan}$ & hh09 & 2.6 & 8.1 & 57.0 & 2.4 & 7.6 & 54.0 & 2.6 & 8.0 & 57.0 \\
\hline 287.80 & .80 & -21.3 & $d+03$ & 3.30 & 2.70 & $\mathrm{pbd}+03$ & & & & ktan & hh09 & 2.6 & 8. & 38.1 & 2.4 & 7.6 & 36.1 & 2.6 & 8. & 38.1 \\
\hline 287.90 & -0.90 & -20.7 & $d+03$ & 5.20 & 2.70 & $\mathrm{pbd}+03$ & & & & ktan & hh09 & 2.6 & 8.1 & 44.3 & 2.5 & 7.6 & 43.2 & 2.6 & 8.0 & 44.3 \\
\hline 287.90 & -0.80 & -25.8 & $d+03$ & 54.10 & 2.70 & $\mathrm{pbd}+03$ & & & & $\mathrm{ktan}$ & hh09 & 2.6 & 8.1 & 96.7 & 2.5 & 7.6 & 94.2 & 2.6 & 8.0 & 96.7 \\
\hline 288.00 & -0.80 & -26.0 & $\mathrm{pbd}+03$ & 60.60 & 2.70 & $\mathrm{pbd}+03$ & 2.6 & 0.25 & rus03 & & & & & 100.4 & & & 100.4 & & & 100.4 \\
\hline 288.20 & -2.90 & -7.4 & rus03 & & & & 3.1 & 0.9 & rus03 & & & & & & & & & & & \\
\hline 289.10 & -0.40 & 23.2 & $\mathrm{pbd}+03$ & 14.00 & 2.70 & $\mathrm{pbd}+03$ & 8.0 & 0.5 & rus03 & & & & & 130.4 & & & 130.4 & & & 130.4 \\
\hline 289.50 & 0.20 & -23.0 & rus03 & & & & 2.9 & 0.2 & rus03 & & & & & & & & & & & \\
\hline 289.80 & -1.20 & 22.0 & $\mathrm{pbd}+03$ & 19.80 & 2.70 & $\mathrm{pbd}+03$ & 8.0 & 0.5 & rus 03 & & & & & 146.3 & & & 146.3 & & & 146.3 \\
\hline 289.80 & 1.10 & 21.9 & $d+03$ & 45.00 & 2.70 & $\mathrm{pbd}+03$ & 8.0 & 0.5 & rus03 & & & & & 192.4 & & & 192.4 & & & 192.4 \\
\hline 289.90 & -0.80 & 22.0 & $\mathrm{pbd}+03$ & 5.00 & 2.70 & $\mathrm{pbd}+03$ & 8.0 & 0.5 & & & & & & 92.5 & & & 92.5 & & & 92.5 \\
\hline 290.40 & -2.90 & -15.0 & rus03 & & & & 4.2 & 0.5 & rus 03 & & & & & 52.0 & & & 52.0 & & & 52.0 \\
\hline 290.40 & 1.50 & -19.0 & rus03 & & & & 2.7 & 0.25 & rus & & & & & & & & & & & \\
\hline & -0.80 & 22.0 & $d+c$ & & 2.70 & & 8.0 & 0.5 & & & & & & 85.9 & & & 85.9 & & & 85.9 \\
\hline .60 & 0.30 & -28.0 & 3 & 4.0 & 2.70 & $\mathrm{pbd}+03$ & 2.8 & 0.25 & rus 03 & & & & & 42.6 & & & 42.6 & & & 42.6 \\
\hline & .80 & 17.0 & 3 & 4.80 & 2.70 & $\mathrm{pbd}+03$ & 7.9 & 0.25 & rus03 & & & & & 90.5 & & & 90.5 & & & 90.5 \\
\hline & -0.30 & 9.0 & +03 & 5.00 & 2.70 & $\mathrm{pbd}+03$ & & & & kout & hh09 & 7.4 & 9.0 & 87.8 & 6.7 & 8.4 & 82.2 & 6.5 & 8.6 & 80.5 \\
\hline 1.30 & -0.70 & -24.5 & +03 & 95.00 & 2.70 & $\mathrm{pbd}+03$ & 2.7 & 0.2 & rus 03 & & & & & 119.6 & & & 119.6 & & & 119.6 \\
\hline & & 6.0 & & 4.00 & 2.70 & $\mathrm{pbd}+03$ & & & & kout & hh09 & 7.2 & 8.9 & 80.0 & 6.5 & 8.2 & 74.7 & 6.3 & 8.5 & 73.2 \\
\hline 291.60 & -0.50 & 10.0 & $\mathrm{pbd}+03$ & 238.00 & 2.70 & $\mathrm{pbd}+03$ & 7.9 & 0.25 & rus03 & & & & & 332.4 & & & 332.4 & & & 332.4 \\
\hline
\end{tabular}


Table A.1. continued.

\begin{tabular}{|c|c|c|c|c|c|c|c|c|c|c|c|c|c|c|c|c|c|c|c|c|}
\hline $\begin{array}{r}l \\
\left({ }^{\circ}\right) \\
(1)\end{array}$ & $\begin{array}{l}b \\
\left({ }^{\circ}\right) \\
(2)\end{array}$ & $\begin{array}{c}V_{\mathrm{lsr}} \\
\mathrm{km} \mathrm{s}^{-1} \\
(3)\end{array}$ & Ref. & $\begin{array}{c}S \\
\text { Jy } \\
(5)\end{array}$ & $\begin{array}{r}\text { Freq } \\
\text { GHz } \\
(6)\end{array}$ & Ref. & $\begin{array}{r}d \\
\mathrm{kpc} \\
(8)\end{array}$ & $\begin{array}{r}d_{\text {erro }} \\
\text { kpc } \\
(9)\end{array}$ & (10) & Mark & $\begin{array}{l}\text { Ref. } \\
\text { (12) }\end{array}$ & $\begin{array}{r}D_{8.5} \\
\mathrm{kpc} \\
(13)\end{array}$ & $\begin{array}{l}R_{8.5} \\
\mathrm{kpc} \\
(14)\end{array}$ & $\begin{array}{r}U_{8.5} \\
\mathrm{pc} \mathrm{cm}^{-2} \\
(15)\end{array}$ & $\begin{array}{l}D_{8.0} \\
\mathrm{kpc} \\
(16)\end{array}$ & $\begin{array}{l}R_{8.0} \\
\mathrm{kpc} \\
(17)\end{array}$ & $\begin{array}{r}U_{8.0} \\
\mathrm{pc} \mathrm{cm}^{-2} \\
(18)\end{array}$ & $\begin{array}{l}D_{8.4} \\
\mathrm{kpc} \\
(19)\end{array}$ & $\begin{array}{l}R_{8.4} \\
\mathrm{kpc} \\
(20)\end{array}$ & $\begin{array}{r}U_{8.4} \\
\mathrm{pc} \mathrm{cm}{ }^{-2} \\
(21)\end{array}$ \\
\hline 1.90 & -0.70 & 25.2 & $d+03$ & 2.90 & 2.70 & $\mathrm{pbd}+03$ & & & & kout & hh09 & 9.2 & 9.9 & 84.7 & 8.3 & 9.1 & 79.1 & 8.1 & 9.3 & 77.8 \\
\hline 1.90 & .00 & 15.0 & $d+03$ & 6.00 & 2.70 & $p b d+03$ & 7.9 & 0.25 & rus03 & & & & & 97.5 & & & 97.5 & & & 97.5 \\
\hline 2.30 & 4.90 & -3.0 & rus03 & & & & 3.3 & 1.0 & rus03 & & & & & 18.0 & & & 18.0 & & & 18.0 \\
\hline 2.40 & 1.60 & -22.4 & rus03 & & & & 2.3 & 0.7 & rus03 & & & & & & & & & & & \\
\hline 3.00 & -1.00 & 66.0 & $\mathrm{~d}+03$ & 2.00 & 2.70 & $\mathrm{pbd}+03$ & & & & kout & hh09 & 13.7 & 13.0 & 97.6 & 12.4 & 11.9 & 91.3 & 12.0 & 11.7 & 89.3 \\
\hline 3.60 & -1.30 & -27.0 & $d+03$ & 5.00 & 2.70 & $\mathrm{pbd}+03$ & 2.5 & 0.5 & rus03 & & & & & 42.6 & & & 42.6 & & & 42.6 \\
\hline 4.80 & 1.80 & -18.0 & $d+03$ & .30 & 2.70 & $\mathrm{pbd}+03$ & 2.2 & 0.25 & rus03 & & & & & 61.4 & & & 61.4 & & & 61.4 \\
\hline 5.10 & -0.60 & 38.0 & $d+03$ & 2.00 & 2.70 & $\mathrm{pbd}+03$ & & & & kout & hh09 & 11.1 & 10.8 & 188.6 & 10.1 & 9.9 & 177.1 & 10.0 & 10.0 & 175.9 \\
\hline 5.10 & -1.60 & -22.0 & $d+03$ & 2.50 & 2.70 & $\mathrm{pbd}+03$ & 2.2 & 0.25 & rus03 & & & & & 53.1 & & & 53.1 & & & 53.1 \\
\hline 5.20 & -0.60 & 51.1 & $d+03$ & 0.00 & 2.70 & $\mathrm{pbd}+03$ & & & & kout & hh09 & 12.5 & 11.8 & 197.7 & 11.4 & 10.8 & 185.9 & 11.1 & 10.7 & 182.6 \\
\hline 5.80 & -0.20 & 17.0 & $d+03$ & 3.10 & 2.70 & $\mathrm{pbd}+03$ & & & & kout & hh09 & 9.3 & 9.5 & 87.2 & 8.5 & 8.7 & 82.1 & 8.4 & 9.0 & 81.5 \\
\hline 6.60 & 1.00 & 25.0 & $d+03$ & 3.00 & 2.70 & $\mathrm{pbd}+03$ & & & & kout & hh09 & 10.2 & 9.9 & 91.7 & 9.3 & 9.2 & 86.2 & 9.2 & 9.3 & 85.6 \\
\hline 7.00 & 0.00 & -28.0 & rus03 & & & & 2.5 & 0.4 & rus 03 & & & & & & & & & & & \\
\hline 7.50 & -0.80 & 23.0 & $\mathrm{pbd}+03$ & 4.00 & 2.70 & & & & & kout & hh09 & 10.2 & 9.8 & 01.0 & 9.3 & 9.1 & 95.0 & 9.3 & 9.3 & 95.0 \\
\hline 7.70 & .00 & 26.0 & & 1.90 & 70 & & & & & & h09 & 10.6 & 10.0 & 80.8 & 9.6 & 9 & 5.6 & 9.6 & .4 & 75.6 \\
\hline 8.20 & & 16.0 & +03 & 3.00 & 2.70 & & & & & & 09 & 9.8 & 9.4 & 9.3 & 8.9 & 8 & 3.7 & 8.9 & .9 & 33.7 \\
\hline 3.20 & & 30.8 & $d+03$ & .00 & 2.70 & & & & & & hh09 & 1.1 & 10.3 & 0.1 & 10.2 & 3 & 7.5 & 10.1 & .6 & 6.1 \\
\hline 3.60 & .10 & 23.0 & $1+03$ & .00 & 2.70 & $1+03$ & & & & kout & hh09 & 10.5 & 9.8 & 2.5 & 9.6 & 1 & 13.7 & 9.6 & .5 & 43.7 \\
\hline 3.80 & .30 & 25.0 & +03 & .00 & 2.70 & $1+03$ & & & & kout & hh09 & 10.7 & 10.0 & 8.8 & 9.8 & 9.2 & 59.2 & 9.8 & .4 & 59.2 \\
\hline 3.90 & -0.40 & 24.5 & $1+03$ & 52.00 & 2.70 & $\mathrm{pbd}+03$ & & & & kout & hh09 & 10.7 & 9.9 & 245.1 & 9.8 & 9.2 & 231.2 & 9.7 & 9.3 & 229.6 \\
\hline 9.00 & 0.10 & 23.0 & $1+03$ & 2.80 & 2.70 & $\mathrm{pbd}+03$ & & & & kout & hh09 & 10.6 & 9.9 & 92.0 & 9.7 & 9.1 & 86.7 & 9.6 & 9.3 & 86.1 \\
\hline 9.40 & -0.30 & -37.0 & $1+03$ & 0.80 & 2.70 & $\mathrm{pbd}+03$ & 5.4 & 0.6 & rus03 & & & & & 38.6 & & & 38.6 & & & 38.6 \\
\hline .50 & -0.20 & 26.0 & $d+03$ & 2.40 & 2.70 & $\mathrm{pbd}+03$ & & & & kout & hh09 & 11.2 & 10.1 & 90.6 & 10.3 & 9.3 & 85.7 & 10.2 & 9.4 & 85.1 \\
\hline . .00 & 1.20 & -46.8 & $1+03$ & 3.00 & 2.70 & $\mathrm{pbd}+03$ & & & & ktan & hh09 & 4.4 & 7.3 & 52.4 & 4.1 & 9 & 0.0 & 4.3 & 2 & 1.6 \\
\hline & 1.00 & 45.1 & +03 & 4.00 & 2.70 & & & & & ktan & hh09 & 4.4 & 7.3 & 57.6 & 4.1 & 9 & 5.0 & 4.3 & . & 56.7 \\
\hline & 10 & -42.0 & 3 & 4.60 & 70 & & & & & ktan & hh09 & 4.5 & 7.2 & 61.3 & 4.2 & 6.8 & 8.5 & 4.4 & 7.1 & 60.4 \\
\hline 2.00 & -0.00 & -27.0 & 3 & 1.40 & 70 & $\mathrm{pbd}+03$ & 2.1 & 0.6 & rus 03 & & & & & 24.8 & & & 24.8 & & & 24.8 \\
\hline & & -24.0 & rus03 & & & & 2.1 & 0.2 & rus03 & & & & & & & & & & & \\
\hline & -0.70 & 31.0 & 3 & 5.40 & 2.70 & & & & & kout & hh09 & 12.2 & 10.4 & 5.7 & 11.1 & 9.6 & 118.0 & 11.1 & 9.7 & 118.0 \\
\hline & & 33.0 & & & 0 & & 2.1 & 0.6 & & & & & & 39.1 & & & 39.1 & & & 39.1 \\
\hline & & -32.0 & & 2.00 & 2.70 & & 2.1 & 0.6 & rus03 & & & & & 27.9 & & & 27.9 & & & 27.9 \\
\hline & .90 & 26.0 & & 3.10 & 2.70 & & & & & kout & hh09 & 1.8 & & 2.2 & .8 & & 96.3 & 10.9 & & 96.9 \\
\hline .50 & .70 & 32.0 & & 9.00 & 2.70 & & & & & kout & hh09 & 12.5 & 10.5 & 51.5 & 11.4 & & 42.5 & 11.4 & & 42.5 \\
\hline .90 & .10 & 26.0 & & 0.30 & 2.70 & & & & & kout & hh09 & 12.0 & 10.1 & 47.4 & 11.0 & & 44.7 & 11.0 & & 44.7 \\
\hline .20 & -0.40 & 45.0 & & 00 & 70 & & & & & ktan & hh09 & 4.9 & 6. & 70.9 & 4.6 & & 88.0 & 4.8 & & 69.9 \\
\hline .40 & & 38.7 & $1+03$ & .30 & 70 & & & & & kfar & pdd04 & 6.2 & 7.0 & 1.8 & 5.5 & 6 & 58.6 & 5.6 & .0 & 60.5 \\
\hline .50 & & 44.7 & & .50 & 70 & & & & & ktan & hh09 & 4.9 & 6. & 9.2 & 4.6 & & 4.3 & 4.9 & & 19.2 \\
\hline .60 & -0.00 & 44.9 & -03 & .00 & 70 & & & & & ktan & hh09 & 4.9 & 6.9 & 119.9 & 4.7 & 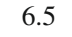 & 6.6 & 4.9 & & 19.9 \\
\hline .70 & & 8.0 & & & & & & & & ktan & hh09 & 5.0 & 6.9 & 1.4 & 4.7 & & 8.5 & 4.9 & & 70.4 \\
\hline & & 3.0 & & 5.00 & 70 & & & & & ktan & hh09 & 5.0 & 6.9 & 67.6 & 4.7 & 6.5 & 4.9 & 4.9 & 6.8 & 66.7 \\
\hline & & 7.0 & & 2.70 & 70 & & 2.3 & 0.25 & rus03 & & & & & 32.8 & & & 2.8 & & & 32.8 \\
\hline & & 6.0 & & & & & & & & kfar & $\operatorname{ch} 87$ & 8.9 & 7.9 & 5.9 & 8.2 & & 1.9 & 8.4 & . & 3.0 \\
\hline & & 7.0 & & & & & & & & kfar & $\operatorname{ch} 87$ & 9.3 & 7.8 & .0 & 8.6 & 7.3 & 7.9 & 8.8 & 7.6 & 8.8 \\
\hline & & 0.0 & & & 70 & $\mathrm{pbd}+03$ & 4.0 & 0.6 & rus03 & & & & & 9.9 & & & 9.9 & & & 79.9 \\
\hline & & 46.4 & & .00 & 70 & & & & & knear & pdd04 & 4.2 & 6.7 & 97.1 & 4.2 & 6.3 & 97.1 & 4.4 & 6.7 & 100.2 \\
\hline & & 47.0 & & & & & 4.0 & 0.6 & & & & & & 54.1 & & & 54.1 & & & 54.1 \\
\hline & & 3.0 & & & & & 4.0 & 6 & & & & & & 49.1 & & & 49.1 & & & 49.1 \\
\hline & & 5.0 & & 5.00 & 70 & & 4.0 & 0.6 & rus 03 & & & & & 58.3 & & & 58.3 & & & 58.3 \\
\hline & .10 & 4.0 & & 6.00 & 70 & & & & & kout & hh09 & 11.5 & 8.9 & 125.2 & 10.6 & 8.2 & 118.6 & 10.9 & 8.4 & 120.8 \\
\hline & & -51.0 & +03 & 4.00 & 70 & $d+03$ & 2.9 & 0.9 & rus03 & & & & & 43.7 & & & 43.7 & & & 43.7 \\
\hline & -0.30 & 36.0 & & 3.00 & 2.70 & $\mathrm{pbd}+03$ & & & & & h09 & 14.7 & 11.1 & 117.0 & 13.5 & 10.2 & 10.5 & 13.6 & 10.2 & 111.1 \\
\hline & & 57.0 & & 2.0 & 70 & & & & & ktan & hh09 & 5.6 & 6.4 & 53.7 & 5.3 & 6.0 & 51.8 & 5.5 & 6.3 & 53.1 \\
\hline .30 & -0.00 & 52.0 & $1+03$ & 5.20 & 70 & $\mathrm{pbd}+03$ & 2.9 & 0.9 & rus03 & & & & & 47.6 & & & 47.6 & & & 47.6 \\
\hline & & 61.9 & & .00 & 70 & & & & & & hh09 & 5.6 & & .9 & 5.3 & & 8.6 & 5.6 & 6.3 & 91.9 \\
\hline & -0.60 & & & 3. & 70 & & & & & kout & hh09 & 14.4 & 10.8 & 115.4 & 13.2 & 9.9 & 108.9 & 13.3 & 10.0 & 109.4 \\
\hline & & 2.3 & & & 0 & & & & & ktan & hh09 & 5.6 & 6.4 & 69.3 & 5.3 & & 6.8 & 5.6 & 6.3 & 69.3 \\
\hline & & 17.2 & $d+03$ & 28.00 & 70 & & & & & kfar & pdd04 & 7.7 & 6.6 & & 7.1 & & 1.7 & 7.5 & 6.6 & 157.3 \\
\hline & & 15.3 & $\mathrm{pbd}+03$ & 9.70 & 2.70 & $\mathrm{pbd}+03$ & & & & kfar & pdd04 & 7.9 & 6.7 & 114.4 & 7.3 & 6.3 & 108.5 & 7.7 & 6.6 & 112.5 \\
\hline & -0.40 & & & & & & 1.5 & 0.4 & & & & & & & & & & & & \\
\hline & & & & & & & & & & & & 11.5 & & & & & & 10.9 & & 110.4 \\
\hline & & -67.0 & & & & & & & & & & 5.9 & & & 5.6 & & 2.9 & 5.9 & & \\
\hline & & 16 & & & & & & & & & & 3.6 & 9 & .5 & 12.6 & & 5.6 & 12.9 & & 76.7 \\
\hline & & 16.0 & & & & & & & & & & 13.9 & 9. & 45.7 & 12.8 & & 43.3 & 13.1 & & 43.9 \\
\hline 316.40 & -0.40 & 3.0 & $\mathrm{pbd}+03$ & 5.00 & 2.70 & $\mathrm{pbd}+03$ & & & & kout & hh09 & 12.7 & 8.8 & 125.9 & 11.8 & 8.2 & 119.9 & 12.1 & 8.4 & 121.9 \\
\hline
\end{tabular}


Table A.1. continued.

\begin{tabular}{|c|c|c|c|c|c|c|c|c|c|c|c|c|c|c|c|c|c|c|c|c|}
\hline $\begin{array}{r}l \\
\left({ }^{\circ}\right) \\
(1) \\
\end{array}$ & $\begin{array}{r}b \\
\left({ }^{\circ}\right) \\
(2) \\
\end{array}$ & $\begin{array}{c}V_{\mathrm{lsr}} \\
\mathrm{km} \mathrm{s}^{-1} \\
(3)\end{array}$ & Ref. & $\begin{array}{r}S \\
\text { Jy } \\
(5)\end{array}$ & $\begin{array}{r}\text { Freq } \\
\text { GHz } \\
(6)\end{array}$ & Ref. & $\begin{array}{r}d \\
\mathrm{kpc} \\
(8)\end{array}$ & $\begin{array}{r}d_{\text {erro }} \\
\text { kpc } \\
(9)\end{array}$ & (10) & Mark & (12) & $\begin{array}{l}D_{8.5} \\
\mathrm{kpc} \\
(13)\end{array}$ & $\begin{array}{c}R_{8.5} \\
\mathrm{kpc} \\
(14)\end{array}$ & $\begin{array}{r}U_{8.5} \\
\mathrm{pc} \mathrm{cm}^{-2} \\
(15)\end{array}$ & $\begin{array}{r}D_{8.0} \\
\mathrm{kpc} \\
(16)\end{array}$ & $\begin{array}{r}R_{8.0} \\
\mathrm{kpc} \\
(17) \\
\end{array}$ & $\begin{array}{r}U_{8.0} \\
\mathrm{pc} \mathrm{cm}{ }^{-2} \\
(18)\end{array}$ & $\begin{array}{r}D_{8.4} \\
\mathrm{kpc} \\
(19)\end{array}$ & $\begin{array}{l}R_{8.4} \\
\mathrm{kpc} \\
(20)\end{array}$ & $\begin{array}{r}U_{8.4} \\
\mathrm{pc} \mathrm{cm}^{-2} \\
(21)\end{array}$ \\
\hline 316.80 & -0.10 & -36.1 & $\mathrm{pbd}+03$ & 37.30 & 2.70 & $\mathrm{pbd}+03$ & & & & knear & pdd04 & 2.5 & 6.9 & 83.2 & 2.4 & 6.5 & 81.0 & 2.6 & 6.9 & 85.4 \\
\hline 316.80 & -0.00 & -36.0 & $d+03$ & 34.00 & 2.70 & $\mathrm{pbd}+03$ & 3.6 & 1.1 & rus03 & & & & & 102.9 & & & 102.9 & & & 102.9 \\
\hline 17.00 & 0.30 & -48.8 & $1+03$ & 17.00 & 2.70 & $d+03$ & 3.6 & 1.1 & rus03 & & & & & 81.7 & & & 81.7 & & & 81.7 \\
\hline 17.30 & 0.20 & -46.8 & 33 & 7.10 & 2.70 & $d+03$ & & & & kfar & gg76 & 9.2 & 6.5 & 114.1 & 8.6 & 6.1 & 109.1 & 9.1 & 6.4 & 113.3 \\
\hline 17.30 & 0.30 & -51.0 & & 2.00 & 2.70 & $d+03$ & 3.6 & 1.1 & rus03 & & & & & 72.7 & & & 72.7 & & & 72.7 \\
\hline 7.60 & -0.40 & 38.0 & $d+03$ & 4.00 & 2.70 & $1+03$ & .6 & 1.1 & rus03 & & & & & 50.4 & & & 50.4 & & & 50.4 \\
\hline 8.00 & 0.80 & -37.0 & $d+03$ & 12.90 & 2.70 & $\mathrm{pbd}+03$ & 3.6 & 1.1 & rus03 & & & & & 74.5 & & & 74.5 & & & 74.5 \\
\hline 8.10 & -0.50 & 3.0 & $d+03$ & 1.10 & 2.70 & $\mathrm{pbd}+03$ & & & & kout & hh09 & 13.1 & 8.8 & 77.6 & 12.1 & 8.2 & 73.6 & 12.5 & 8.4 & 75.2 \\
\hline 9.20 & -0.40 & -24.7 & $d+03$ & 12.30 & 2.70 & $\mathrm{pbd}+03$ & & & & kfar & gg76 & 11.2 & 7.3 & 156.3 & 10.4 & 6.8 & 148.8 & 10.9 & 7.1 & 53.5 \\
\hline 9.40 & -0.00 & -14.1 & $d+03$ & 12.00 & 2.70 & $\mathrm{pbd}+03$ & & & & kfar & gg76 & 12.0 & 7.8 & 162.3 & 11.2 & 7.3 & 155.0 & 11.6 & 7.5 & 158.7 \\
\hline 19.87 & 0.77 & -38.0 & ch87 & 1.60 & 5.00 & ch87 & & & & knear & ch87 & 2.6 & 6.7 & 30.5 & 2.5 & 6.3 & 29.7 & 2.6 & 6.7 & 30.5 \\
\hline 20.11 & -0.51 & -13.0 & ch87 & 4.00 & 5.00 & ch87 & & & & kfar & ch87 & 12.2 & 7.9 & 116.1 & 11.4 & 7.3 & 111.0 & 11.8 & 7.6 & 113.5 \\
\hline 20.20 & 0.80 & -36.0 & $b d+03$ & 10.00 & 2.70 & $\mathrm{pbd}+03$ & & & & knear & pdd04 & 2.4 & 6.8 & 52.2 & 2.4 & 6.4 & 52.2 & 2.5 & 6.8 & 53.6 \\
\hline 20.24 & 0.42 & -31.0 & ch87 & 2.30 & 5.00 & ch87 & & & & knear & ch87 & 2.1 & 7.0 & 29.9 & 2.0 & 6.6 & 28.9 & 2.2 & 7.0 & 30.8 \\
\hline 20.30 & -0.20 & -9.2 & $b d+03$ & 12.00 & 2.70 & $\mathrm{pbd}+03$ & & & & kfar & gg76 & 12.5 & 8.1 & 166.8 & 11.7 & 7.5 & 159.6 & 12.1 & 7.7 & 163.2 \\
\hline 20.38 & 0.14 & -3.0 & ch87 & 3.60 & 5.00 & ch87 & & & & kfar & $\operatorname{ch} 87$ & 13.0 & 8.4 & 17.0 & 12.1 & 7.8 & 11.5 & 12.5 & 8.1 & 114.0 \\
\hline 20.40 & -1.00 & -50.0 & rus03 & & & & 3.3 & 0.25 & rus03 & & & & & 113.0 & & & 113.0 & & & 113.0 \\
\hline 20.71 & 0.20 & -7.0 & ch87 & 14.30 & 5.00 & ch87 & & & & kfar & ch87 & 12.8 & 8.2 & 83.3 & 11.9 & 7.6 & 4.6 & 12.3 & 7.8 & 178.5 \\
\hline 21.00 & -0.50 & -61.3 & $d+03$ & .10 & 2.70 & $\mathrm{pbd}+03$ & & & & knear & gg76 & 4.1 & 5 & 77.3 & 3.9 & & 1.8 & 4.0 & 6 & 76.0 \\
\hline 1.10 & -0.50 & 5.7 & & .00 & 2.70 & & & & & near & pdd04 & 3.7 & 6.1 & 89.3 & 3.5 & 5. & & 3.6 & 6.1 & 87.7 \\
\hline 2.20 & 0.60 & -51.9 & +03 & 14.00 & 2.70 & & & & & knear & pdd04 & 3.4 & 6.2 & 73.7 & 3.3 & 5.8 & 72.2 & 3.4 & 6.2 & 73.7 \\
\hline 4.10 & 0.20 & 91.0 & +03 & 4.30 & 2.70 & & & & & ktan & hh09 & 6.9 & 5.0 & 79.7 & & & & & & \\
\hline 4.20 & 0.10 & -89.2 & +03 & 4.80 & 2.70 & & & & & knear & pdd04 & 6.5 & 5.0 & 79.4 & 5.8 & 4.7 & 73.6 & 5.6 & 5.1 & 71.9 \\
\hline 5.00 & -0.60 & 25.0 & +03 & 3.50 & 2.70 & $\mathrm{pbd}+03$ & & & & kout & hh09 & 16.7 & 10.9 & 134.1 & 15.4 & 10.0 & 127.0 & 15.6 & 10.0 & 128.1 \\
\hline 6.20 & 1.00 & -42.0 & +03 & 6.00 & 2.70 & +03 & 2.3 & 0.4 & rus03 & & & & & 42.8 & & & 42.8 & & & 42.8 \\
\hline 26.30 & 0.70 & -45.0 & +03 & 14.00 & 2.70 & & 2.3 & 0.4 & rus03 & & & & & 56.8 & & & 56.8 & & & 56.8 \\
\hline 6.40 & 0.90 & -39.0 & +03 & 16.00 & 2.70 & & 2.3 & 0.4 & rus03 & & & & & 59.4 & & & 59.4 & & & 59.4 \\
\hline 5.50 & 0.9 & -41.3 & +03 & 9.20 & 2.70 & & & & & knear & gg76 & 2.6 & 6.5 & 53.6 & 2.7 & 6.0 & 55.0 & 2.8 & 6.4 & 56.3 \\
\hline 6.60 & 0.60 & 44.0 & $\mathrm{bd}+03$ & 37.10 & 2.70 & & 2.3 & 0.4 & rus03 & & & & & 78.6 & & & 78.6 & & & 78.6 \\
\hline 6.60 & -1.00 & 21.0 & rus 03 & & & & 0.9 & 0.3 & rus03 & & & & & & & & & & & \\
\hline 6.70 & 0.60 & -44.5 & $b d+03$ & 0.00 & 2.70 & & & & & knear & pdd04 & 3.0 & 6.2 & 03.6 & 2.8 & 5.8 & & 2.9 & 6.3 & 01.3 \\
\hline & -0.60 & -48.8 & & 1.60 & 70 & & & & & ear & Id 04 & 3.2 & 6 & 1.9 & 3.1 & 5. & & 3.1 & 1 & 19.3 \\
\hline & & -48.0 & & 54.00 & 70 & & & & & near & $\mathrm{dd} 04$ & 3.2 & 6.1 & 11.0 & 3.0 & 5.7 & 06.3 & 3.1 & 6.1 & 08.7 \\
\hline & -0.80 & -37.0 & & & & & 2.8 & 0.5 & rus03 & & & & & & & & & & & \\
\hline . 00 & -0.10 & -44.8 & $\mathrm{pbd}+\mathrm{c}$ & 8.00 & 2.70 & & & & & knear & pdd04 & 3.0 & 6.2 & 56.3 & 2.9 & 5.8 & 55.0 & 3.0 & 6.2 & 56.3 \\
\hline 3.00 & -0.60 & -45.6 & $\mathrm{~s} 03$ & & & & 3.0 & 0.4 & rus & & & & & & & & & & & \\
\hline 3.10 & -0.80 & 18.5 & s0 & & & & & 0.3 & & & & & & & & & & & & \\
\hline 8.20 & -2.00 & -25.0 & is 03 & & & & 0.8 & 0.2 & rus03 & & & & & & & & & & & \\
\hline 28.30 & 0.40 & -96.6 & $d+03$ & 8.00 & 2.70 & $\mathrm{pbd}+03$ & & & & kfar & pdd04 & 8.3 & 4.6 & 110.9 & 8.0 & 4.4 & 108.2 & 8.7 & 4.7 & 114.4 \\
\hline 28.30 & -0.60 & 43.0 & & .00 & 2.70 & $\mathrm{pbd}+03$ & 3.3 & 0.7 & rus03 & & & & & 43.2 & & & 43.2 & & & 43.2 \\
\hline 8.60 & -0.50 & 51.0 & & 9.00 & 2.70 & $\mathrm{pbd}+03$ & & & & knear & pdd04 & 3.4 & 5.9 & 63.6 & 3.2 & 5.5 & 61.1 & 3.3 & 6.0 & 62.3 \\
\hline .40 & & 107. & & & 70 & & & & & $\tan$ & & 7.3 & & 87.0 & & & & & & \\
\hline .90 & -0.40 & 56.1 & & .20 & 70 & & & & & hear & pdd04 & 3.7 & 5.6 & 76.5 & 3.5 & 5.3 & 7 & 3.5 & 5.7 & 73.7 \\
\hline .30 & -0.30 & 64.2 & & .00 & 70 & & & & & knear & pdd04 & 4.1 & 5.3 & 89.1 & 3.9 & $5 .($ & & 3.9 & 5.4 & 86.2 \\
\hline .50 & .10 & 8.8 & & 7.00 & 70 & $\mathrm{pbd}+03$ & & & & kfar & pdd04 & 9.6 & 4.6 & 220.4 & 9.1 & 4.3 & 212.7 & 9.8 & 4.7 & 223.5 \\
\hline & .40 & 5.0 & & 12.00 & 70 & $\mathrm{pbd}+03$ & 3.8 & 0.5 & rus03 & & & & & 75.4 & & & 75.4 & & & 75.4 \\
\hline & & 5.0 & & 14.50 & .00 & & & & & knear & ch87 & 3.7 & 5.5 & 80.5 & 3.4 & 5.2 & 76.1 & 3.5 & 5.6 & 77.6 \\
\hline & & 3.0 & $d$ & & 70 & & 8 & 0.5 & & & & & & 4.7 & & & & & & 44.7 \\
\hline & & 7.4 & & & & & & & & & & & & 122.9 & & & 122 & & & 122.9 \\
\hline & & 1.0 & & & 70 & & 3 & 0.5 & & & & & & 50.0 & & & 50 & & & 50.0 \\
\hline & 0.60 & 57.1 & & 5.40 & .70 & & 3.8 & 0.5 & rus03 & & & & & 83.7 & & & 83.7 & & & 83.7 \\
\hline & -0.40 & 3.8 & & 42.20 & 2.70 & & & & & kfar & pdd04 & 11.5 & 5.5 & 239.9 & 10.9 & 5.2 & 231.5 & 11.5 & 5.5 & 239.9 \\
\hline .00 & 0.8 & 52.0 & & & 70 & & & 0.5 & & & & & & 56.3 & & & 56 & & & 56.3 \\
\hline 3.00 & 2.00 & 20.0 & & 00 & 70 & & & 0 & & & & & & 35.0 & & & 35 & & & 35.0 \\
\hline 3.10 & -0.40 & 54.5 & & .00 & 70 & & 3 & 0 & & & & & & 122.9 & & & 122.9 & & & 122.9 \\
\hline & -0.40 & 50.1 & & .00 & 70 & & 3.8 & 0.5 & rus03 & & & & & 28.9 & & & 128.9 & & & 128.9 \\
\hline 33.60 & -0.20 & -47.6 & $1+03$ & 122.00 & 70 & $\mathrm{pbd}+03$ & & & & knear & pdd04 & 3.3 & 5.7 & 148.7 & 3.1 & 5.4 & 142.6 & 3.2 & 5.8 & 145.7 \\
\hline 33.70 & -0.50 & -49.9 & $1+03$ & 5.60 & 2.70 & $\mathrm{pbd}+03$ & 3.8 & 0.5 & rus03 & & & & & 58.5 & & & 58.5 & & & 58.5 \\
\hline 34.70 & -0.70 & 16.0 & & 1.50 & 2.70 & $\mathrm{pbd}+03$ & & & & kout & hh09 & 17.5 & 10.5 & 104.3 & 16.2 & 9.6 & 99.1 & 16.6 & 9.6 & 100.7 \\
\hline 35.80 & -0.20 & 52.1 & $d+03$ & 9.90 & 2.70 & $\mathrm{pbd}+03$ & & & & knear & gg76 & 3.7 & 5.4 & 69.5 & 3.4 & 5.1 & 5.7 & 3.5 & 5.5 & 67.0 \\
\hline 36.40 & 0.20 & 87.2 & $1+03$ & 35.00 & 2.70 & $\mathrm{pbd}+03$ & & & & knear & pdd04 & 5.3 & 4.2 & 134.5 & 4.9 & 4.0 & 127.6 & 4.9 & 4.4 & 127.6 \\
\hline & -1.50 & 24.9 & $\mathrm{a}+\mathbf{0}$ & 6.00 & 2.70 & $\mathrm{pbd}+03$ & 1.4 & 0.25 & rus03 & & & & & 30.8 & & & 30.8 & & & 30.8 \\
\hline & 0.00 & 79.0 & & 70.00 & 2.70 & $\mathrm{pbd}+03$ & & & & & pdd04 & 10.7 & & 270.6 & 10.1 & & 260.4 & 10.8 & T. & 272.3 \\
\hline 336.90 & -0.10 & -73.1 & $\mathrm{pbd}+03$ & 20.50 & 2.70 & $\mathrm{pbd}+03$ & & & & knear & gg76 & 4.7 & 4.6 & 103.8 & 4.4 & 4.3 & 99.3 & 4.4 & 4.8 & 99.3 \\
\hline
\end{tabular}


Table A.1. continued.

\begin{tabular}{|c|c|c|c|c|c|c|c|c|c|c|c|c|c|c|c|c|c|c|c|c|}
\hline $\begin{array}{r}l \\
\left({ }^{\circ}\right) \\
(1)\end{array}$ & $\begin{array}{l}b \\
\left({ }^{\circ}\right) \\
(2)\end{array}$ & $\begin{array}{c}V_{\mathrm{lsr}} \\
\mathrm{km} \mathrm{s}^{-1} \\
(3)\end{array}$ & Ref. & $\begin{array}{c}S \\
\text { Jy } \\
(5)\end{array}$ & $\begin{array}{r}\text { Freq } \\
\text { GHz } \\
(6)\end{array}$ & Ref. & $\begin{array}{r}d \\
\mathrm{kpc} \\
(8)\end{array}$ & $\begin{array}{r}d_{\text {erro }} \\
\text { kpc } \\
(9)\end{array}$ & (10) & Mark & Ref. & $\begin{array}{l}D_{8.5} \\
\text { kpc } \\
(13)\end{array}$ & $\begin{array}{l}R_{8.5} \\
\mathrm{kpc} \\
(14)\end{array}$ & $\begin{array}{r}U_{8.5} \\
\mathrm{pc} \mathrm{cm}^{-2} \\
(15)\end{array}$ & $\begin{array}{c}D_{8.0} \\
\mathrm{kpc} \\
(16)\end{array}$ & $\begin{array}{l}R_{8.0} \\
\mathrm{kpc} \\
(17)\end{array}$ & $\begin{array}{r}U_{8.0} \\
\mathrm{pc} \mathrm{cm}^{-2} \\
(18)\end{array}$ & $\begin{array}{c}D_{8.4} \\
\mathrm{kpc} \\
(19)\end{array}$ & $\begin{array}{l}R_{8.4} \\
\mathrm{kpc} \\
(20)\end{array}$ & 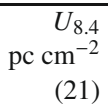 \\
\hline 337.10 & -0.20 & $\begin{array}{l}-72.8 \\
\end{array}$ & $\mathrm{pbd}+03$ & 30.00 & 2.70 & $\mathrm{pbd}+03$ & & & & kfar & pdd04 & 11.0 & 4.5 & 207.8 & 10.4 & 4.3 & 200.2 & 11.1 & 4.6 & 209.1 \\
\hline 337.60 & 0.00 & -54.8 & $\mathrm{pbd}+03$ & 6.50 & 2.70 & $\mathrm{pbd}+03$ & & & & kfar & gg76 & 11.8 & 5.1 & 130.8 & 11.2 & 4.8 & 126.3 & 11.8 & 5.1 & 130.8 \\
\hline 337.90 & -0.50 & -40.2 & $\mathrm{pbd}+03$ & 16.00 & 2.70 & $\mathrm{pbd}+03$ & & & & knear & pdd04 & 3.1 & 5.7 & 72.4 & 2.9 & 5.4 & 69.3 & 3.0 & 5.8 & 70.8 \\
\hline 338.00 & -0.10 & -47.9 & $\mathrm{pbd}+03$ & 50.00 & 2.70 & $\mathrm{pbd}+03$ & & & & kfar & gg76 & 12.2 & 5.4 & 264.0 & 11.5 & 5.1 & 253.8 & 12.2 & 5.3 & 264.0 \\
\hline 338.10 & -0.10 & -41.7 & $\mathrm{pbd}+03$ & 7.80 & 2.70 & $\mathrm{pbd}+03$ & & & & kfar & gg76 & 12.5 & 5.6 & 144.4 & 11.8 & 5.3 & 139.0 & 12.5 & 5.6 & 144.4 \\
\hline 338.40 & 0.00 & -36.9 & $\mathrm{pbd}+03$ & 49.60 & 2.70 & $\mathrm{pbd}+03$ & & & & kfar & gg76 & 12.8 & 5.8 & 271.9 & 12.1 & 5.5 & 261.9 & 12.7 & 5.8 & 270.5 \\
\hline 338.70 & 0.60 & -62.0 & $\mathrm{pbd}+03$ & 4.20 & 2.70 & $\mathrm{pbd}+03$ & 5.2 & 1.6 & rus03 & & & & & 65.5 & & & 65.5 & & & 65.5 \\
\hline 338.90 & 0.60 & -63.0 & $\mathrm{pbd}+03$ & 11.00 & 2.70 & $\mathrm{pbd}+03$ & 5.2 & 1.6 & rus 03 & & & & & 90.3 & & & 90.3 & & & 90.3 \\
\hline 338.90 & -0.10 & -40.0 & $\mathrm{pbd}+03$ & 3.00 & 2.70 & $\mathrm{pbd}+03$ & 2.7 & 0.6 & rus 03 & & & & & 37.8 & & & 37.8 & & & 37.8 \\
\hline 339.10 & -0.40 & -37.0 & $\mathrm{pbd}+03$ & 4.00 & 2.70 & $\mathrm{pbd}+03$ & 2.7 & 0.6 & rus03 & & & & & 41.6 & & & 41.6 & & & 41.6 \\
\hline 339.58 & -0.12 & -30.0 & ch87 & 3.40 & 5.00 & ch87 & & & & kfar & ch87 & 13.3 & 6.1 & 116.5 & 12.5 & 5.8 & 111.8 & 13.2 & 6.0 & 115.9 \\
\hline 340.30 & -0.20 & -43.2 & $\mathrm{pbd}+03$ & 9.00 & 2.70 & $\mathrm{pbd}+03$ & & & & kfar & gg76 & 12.5 & 5.4 & 151.5 & 11.8 & 5.1 & 145.8 & 12.5 & 5.3 & 151.5 \\
\hline 340.80 & -1.00 & -24.9 & $\mathrm{pbd}+03$ & 18.00 & 2.70 & $\mathrm{pbd}+03$ & 2.5 & 0.4 & rus03 & & & & & 65.3 & & & 65.3 & & & 65.3 \\
\hline 341.00 & -0.10 & -38.0 & $\mathrm{pbd}+03$ & 7.00 & 2.70 & $\mathrm{pbd}+03$ & 3.4 & 1.0 & rus 03 & & & & & 58.5 & & & 58.5 & & & 58.5 \\
\hline 341.30 & -0.30 & -38.0 & $\mathrm{pbd}+03$ & 5.60 & 2.70 & $\mathrm{pbd}+03$ & 3.4 & 1.0 & rus 03 & & & & & 54.3 & & & 54.3 & & & 54.3 \\
\hline 341.96 & 0.20 & -8.0 & ch87 & 2.80 & 5.00 & $\operatorname{ch} 87$ & & & & kfar & ch87 & 15.3 & 7.7 & 119.9 & 14.3 & 7.2 & 114.6 & 14.9 & 7.3 & 117.8 \\
\hline 343.50 & -0.00 & -30.2 & $\mathrm{pbd}+03$ & 15.00 & 2.70 & $\mathrm{pbd}+03$ & & & & kfar & gg76 & 13.3 & 5.7 & 187.2 & 12.6 & 5.4 & 180.6 & 13.2 & 5.6 & 186.3 \\
\hline 343.90 & -4.70 & & rus03 & & & & 2.0 & 0.6 & rus03 & & & & & & & & & & & \\
\hline 345.00 & 1.50 & -14.8 & $\mathrm{pbd}+03$ & 91.00 & 2.70 & $\mathrm{pbd}+03$ & 1.9 & 0.25 & rus 03 & & & & & 93.3 & & & 93.3 & & & 93.3 \\
\hline 345.20 & 1.00 & -9.2 & $p b d+03$ & 23.10 & 2.70 & $\mathrm{pbd}+03$ & 1.9 & 0.25 & rus03 & & & & & 59.1 & & & 59.1 & & & 59.1 \\
\hline 345.30 & 1.50 & -15.3 & $\mathrm{pbd}+03$ & 17.10 & 2.70 & $\mathrm{pbd}+03$ & 1.9 & 0.25 & rus03 & & & & & 53.4 & & & 53.4 & & & 53.4 \\
\hline 345.40 & -0.90 & -23.0 & $\mathrm{pbd}+03$ & 33.00 & 2.70 & $\mathrm{pbd}+03$ & & & & knear & pdd04 & 2.6 & 6.0 & 82.0 & 2.5 & 5.7 & 79.9 & 2.5 & 6.1 & 79.9 \\
\hline 345.40 & 1.40 & -14.8 & $\mathrm{pbd}+03$ & 42.80 & 2.70 & $\mathrm{pbd}+03$ & 1.9 & 0.25 & rus03 & & & & & 72.6 & & & 72.6 & & & 72.6 \\
\hline 345.49 & 0.31 & -22.3 & swa+04 & 2.47 & 4.86 & swa+04 & & & & knear & swa +04 & 2.6 & 6.1 & 35.2 & 2.4 & 5.7 & 33.4 & 2.5 & 6.1 & 34.3 \\
\hline 345.50 & 0.30 & -20.0 & $\mathrm{pbd}+03$ & 4.70 & 2.70 & $\mathrm{pbd}+03$ & 2.0 & 0.25 & rus03 & & & & & 36.0 & & & 36.0 & & & 36.0 \\
\hline 345.50 & 0.20 & -13.0 & $\mathrm{pbd}+03$ & 2.10 & 2.70 & $\mathrm{pbd}+03$ & 1.9 & 0.25 & rus 03 & & & & & 26.6 & & & 26.6 & & & 26.6 \\
\hline 345.55 & -0.04 & -6.0 & ch87 & 16.00 & 5.00 & ch87 & & & & kfar & ch87 & 15.7 & 7.7 & 218.1 & 14.7 & 7.2 & 208.7 & 15.3 & 7.4 & 214.4 \\
\hline 345.83 & 0.04 & -10.0 & ch87 & 2.10 & 5.00 & ch87 & & & & kfar & ch87 & 15.2 & 7.2 & 108.5 & 14.2 & 6.7 & 103.7 & 14.9 & 6.9 & 107.1 \\
\hline 346.50 & 0.10 & 2.0 & $\mathrm{pbd}+03$ & 1.00 & 2.70 & $\mathrm{pbd}+03$ & & & & kout & hh09 & 17.0 & 9.0 & 89.4 & 15.9 & 8.3 & 85.5 & 16.4 & 8.4 & 87.3 \\
\hline 347.70 & 1.90 & -19.0 & rus03 & & & & 2.0 & 0.6 & rus03 & & & & & & & & & & & \\
\hline 348.20 & -1.00 & -18.0 & $\mathrm{pbd}+03$ & 12.60 & 2.70 & $\mathrm{pbd}+03$ & 2.9 & 0.25 & rus03 & & & & & 64.0 & & & 64.0 & & & 64.0 \\
\hline 348.20 & 0.40 & -12.0 & rus03 & 8.30 & 5.00 & ch87 & 1.3 & 0.4 & rus03 & knear & ch87 & 1.8 & 6.7 & 33.3 & & & 33.3 & & & 33.3 \\
\hline 348.23 & -0.97 & -17.9 & swa+04 & 7.95 & 4.86 & swa +04 & & & & kfar & swa +04 & 14.2 & 6.1 & 161.4 & 13.3 & 5.7 & 154.5 & 14.0 & 6.0 & 159.9 \\
\hline 348.60 & -0.60 & -19.0 & $\mathrm{pbd}+03$ & 17.60 & 2.70 & $\mathrm{pbd}+03$ & 2.9 & 0.25 & rus03 & & & & & 71.5 & & & 71.5 & & & 71.5 \\
\hline 348.70 & -1.00 & -12.6 & $p b d+03$ & 39.20 & 2.70 & $\mathrm{pbd}+03$ & 2.9 & 0.25 & rus03 & & & & & 93.4 & & & 93.4 & & & 93.4 \\
\hline 349.80 & -0.60 & -25.8 & $\mathrm{pbd}+03$ & 10.40 & 2.70 & $\mathrm{pbd}+03$ & 2.2 & 0.3 & rus03 & knear & gg76 & 3.5 & 5.1 & 49.9 & & & 49.9 & & & 49.9 \\
\hline 349.83 & -0.53 & -25.7 & swa+04 & 3.50 & 4.86 & swa+04 & 2.2 & 0.3 & rus03 & knear & swa+04 & 3.5 & 5.1 & 35.4 & & & 35.4 & & & 35.4 \\
\hline 350.00 & 0.20 & -5.0 & rus03 & & & & 1.6 & 0.5 & rus03 & & & & & & & & & & & \\
\hline 350.50 & 1.00 & -10.9 & $\mathrm{pbd}+03$ & 4.00 & 2.70 & $\mathrm{pbd}+03$ & 1.9 & 0.2 & rus03 & & & & & 32.9 & & & 32.9 & & & 32.9 \\
\hline 351.00 & 0.70 & & $\mathrm{pbd}+03$ & 50.00 & 2.70 & $\mathrm{pbd}+03$ & 1.9 & 0.2 & rus03 & & & & & 76.4 & & & 76.4 & & & 76.4 \\
\hline 351.20 & 0.50 & -7.1 & $\mathrm{pbd}+03$ & 21.00 & 2.70 & $\mathrm{pbd}+03$ & 1.9 & 0.2 & rus03 & & & & & 57.2 & & & 57.2 & & & 57.2 \\
\hline 351.40 & 0.70 & -3.1 & $\mathrm{pbd}+03$ & 176.00 & 2.70 & $\mathrm{pbd}+03$ & 1.9 & 0.2 & rus03 & & & & & 116.3 & & & 116.3 & & & 116.3 \\
\hline 351.40 & 0.60 & & $\mathrm{pbd}+03$ & 190.00 & 2.70 & $\mathrm{pbd}+03$ & 1.9 & 0.2 & rus03 & & & & & 119.3 & & & 119.3 & & & 119.3 \\
\hline 351.60 & -1.30 & -14.0 & $\mathrm{pbd}+03$ & 35.30 & 2.70 & $\mathrm{pbd}+03$ & & & & knear & pdd04 & 2.6 & 5.9 & 83.9 & 2.5 & 5.6 & 81.7 & 2.5 & 6.0 & 81.7 \\
\hline 351.70 & -1.20 & -13.0 & $\mathrm{pbd}+03$ & 43.00 & 2.70 & $\mathrm{pbd}+03$ & & & & knear & gg76 & 2.5 & 6.0 & 87.3 & 2.4 & 5.7 & 85.0 & 2.4 & 6.1 & 85.0 \\
\hline 351.70 & 0.50 & -2.3 & $\mathrm{pbd}+03$ & 2.40 & 2.70 & $\mathrm{pbd}+03$ & 1.9 & 0.2 & rus03 & & & & & 27.8 & & & 27.8 & & & 27.8 \\
\hline 352.40 & 2.10 & -7.0 & rus03 & & & & 1.0 & 0.2 & rus03 & & & & & 40.0 & & & 40.0 & & & 40.0 \\
\hline 352.80 & 1.30 & 1.4 & $p b d+03$ & 14.90 & 2.70 & $\mathrm{pbd}+03$ & & & & kout & hh09 & 17.5 & 9.1 & 224.3 & 16.3 & 8.4 & 213.9 & 16.8 & 8.4 & 218.3 \\
\hline 353.00 & 0.90 & -4.0 & $\mathrm{pbd}+03$ & 37.20 & 2.70 & $\mathrm{pbd}+03$ & 2.0 & 0.25 & rus03 & & & & & 71.7 & & & 71.7 & & & 71.7 \\
\hline 353.10 & 0.40 & -3.3 & $\mathrm{pbd}+03$ & 56.70 & 2.70 & $\mathrm{pbd}+03$ & 2.0 & 0.25 & rus03 & & & & & 82.5 & & & 82.5 & & & 82.5 \\
\hline 353.10 & 0.70 & -3.8 & $\mathrm{pbd}+03$ & 269.00 & 2.70 & $\mathrm{pbd}+03$ & 2.0 & 0.25 & rus 03 & & & & & 138.6 & & & 138.6 & & & 138.6 \\
\hline 353.20 & 0.90 & -4.4 & $\mathrm{pbd}+03$ & 145.20 & 2.70 & $\mathrm{pbd}+03$ & 2.0 & 0.25 & rus03 & & & & & 112.8 & & & 112.8 & & & 112.8 \\
\hline 353.42 & -0.37 & -16.1 & tlhj08 & 7.00 & 2.70 & $\mathrm{pbd}+03$ & & & & knear & tlhj08 & 3.4 & 5.2 & 58.1 & 3.1 & 4.9 & 56.3 & 3.2 & 4.8 & 56.9 \\
\hline 355.10 & 20.90 & 4.5 & rus 03 & & & & 0.2 & 0.04 & rus03 & & & & & & & & & & & \\
\hline 355.60 & 0.80 & 6.5 & rus03 & & & & 1.2 & 0.25 & rus03 & & & & & & & & & & & \\
\hline 359.50 & -0.60 & 18.0 & rus03 & & & & 1.5 & 0.2 & rus03 & & & & & 20.0 & 1.5 & 6.5 & 20.0 & & & 20.0 \\
\hline
\end{tabular}

References: ab09: Anderson \& Bania (2009); ahck02: Araya et al. (2002); bdc01: Blum et al. (2001); brm+08: Brunthaler et al. (2008); ccg+98: Condon et al. (1998); cf08: Crowther \& Furness (2008); cor+07: Copetti et al. (2007); ch87: Caswell \& Haynes (1987); gg76: Georgelin \& Georgelin (1976); GB6: Gregory et al. (1996); hh09: This work; kjb+03: Kolpak et al. (2003); kb94: Kuchar \& Bania (1994); lt08: Leahy \& Tian (2008); mrm+08: Moscadelli et al. (2008); pbd+03: Paladini et al. (2003); pdd04: Paladini et al. (2004); pmg08: Pandian et al. (2008); rus03: Russeil (2003); rag07: Russeil et al. (2007); rmb+08: Reid et al. (2008); swa+04: Sewilo et al. (2004); thj08: Tian et al. (2008); was+03: Watson et al. (2003); PMN: Wright et al. (1994, 1996); Griffith et al. (1994, 1995); xrm+08: Xu et al. (2008); zzr+08: Zhang et al. (2008). 
Table A.2. GMCs as tracers of spiral arms. Columns 1 and 2 are the Galactic longitude and latitude; Cols. 3 to 6 list velocity, the luminosity of $\mathrm{CO}$ emission line (in unit of $10^{3} \mathrm{~K} \mathrm{~km} \mathrm{~s}^{-1} \mathrm{pc}^{2}$ ), distance and mass of molecular cloud, which were all taken from the reference given in Col. 8; Col. 7 is the note for distance ambiguity given in this reference, used as the same convention as in Table A.1; Cols. 9 and 10 list the distances to the Sun and to the Galactic center, which are estimated by the velocity and the rotation curve with $R_{0}=8.5 \mathrm{kpc}$ and $\Theta_{0}=220 \mathrm{~km} \mathrm{~s}{ }^{-1}$ when the stellar distance is unavailable; Col. 11 gives the mass of molecular clouds re-scaled by the newly estimated distance; Cols. $12-14$ are the same as Cols. $9-11$ but with $R_{0}=8.0 \mathrm{kpc}$ and $\Theta_{0}=220 \mathrm{~km} \mathrm{~s}^{-1}$. Columns $15-17$ are the same as Cols. $9-11$ but with $R_{0}=8.4 \mathrm{kpc}$ and $\Theta_{0}=254 \mathrm{~km} \mathrm{~s}{ }^{-1}$.

\begin{tabular}{|c|c|c|c|c|c|c|c|c|c|c|c|c|c|c|c|c|}
\hline $\begin{array}{r}l \\
\left({ }^{\circ}\right) \\
(1)\end{array}$ & $\begin{array}{r}b \\
\left({ }^{\circ}\right) \\
(2)\end{array}$ & $\begin{array}{r}V_{\text {lsr }} \\
\mathrm{km} \mathrm{s}^{-1} \\
(3)\end{array}$ & $\begin{array}{r}L_{\mathrm{CO}} \\
10^{3} \mathrm{~K} \mathrm{~km} \mathrm{~s}^{-1} \mathrm{pc}^{2} \\
(4)\end{array}$ & $\begin{array}{r}D \\
\mathrm{kpc} \\
(5)\end{array}$ & $\begin{array}{r}M_{\mathrm{GMCs}} \\
10^{5} M_{\odot} \\
(6)\end{array}$ & Mark & Ref. & $\begin{array}{r}D_{8.5} \\
\mathrm{kpc} \\
(9)\end{array}$ & $\begin{array}{l}R_{8.5} \\
\mathrm{kpc} \\
(10)\end{array}$ & $\begin{array}{r}M_{8.5} \\
10^{5} M_{\odot} \\
(11)\end{array}$ & $\begin{array}{l}D_{8.0} \\
\mathrm{kpc} \\
(12)\end{array}$ & $\begin{array}{l}R_{8.0} \\
\mathrm{kpc} \\
(13)\end{array}$ & $\begin{array}{r}M_{8.0} \\
10^{5} M_{\odot} \\
(14)\end{array}$ & $\begin{array}{l}D_{8.4} \\
\mathrm{kpc} \\
(15)\end{array}$ & $\begin{array}{l}R_{8.4} \\
\mathrm{kpc} \\
(16)\end{array}$ & $\begin{array}{r}M_{8.4} \\
10^{5} M_{\odot} \\
(17)\end{array}$ \\
\hline 8.00 & -0.50 & 128.00 & 72.70 & 10.10 & 4.435 & ktan & srby87 & & & & & & & & & \\
\hline 8.20 & 0.20 & 20.00 & 1402.00 & 15.90 & 85.522 & kfar & srby 87 & 13.4 & 5.2 & 60.74 & 12.7 & 4.9 & 54.56 & 13.4 & 5.2 & 60.74 \\
\hline 8.30 & -0.10 & 48.00 & 50.20 & 13.20 & 3.062 & kfar & srby 87 & 11.5 & 3.3 & 2.32 & 10.9 & 3.2 & 2.09 & 11.6 & 3.4 & 2.36 \\
\hline 8.30 & -0.30 & 16.00 & 28.10 & 3.20 & 1.714 & knear & srby 87 & 2.9 & 5.6 & 1.41 & 2.7 & 5.3 & 1.22 & 2.8 & 5.8 & 1.31 \\
\hline 8.40 & -0.30 & 37.00 & 233.00 & 5.70 & 14.213 & knear & srby 87 & 4.7 & 3.9 & 9.66 & 4.4 & 3.7 & 8.47 & 4.4 & 4.1 & 8.47 \\
\hline 8.50 & -1.00 & 16.00 & 106.00 & 3.20 & 6.466 & knear & srby87 & 2.9 & 5.7 & 5.31 & 2.7 & 5.4 & 4.60 & 2.7 & 5.8 & 4.60 \\
\hline 8.70 & 0.60 & 22.00 & 8.60 & 4.00 & 0.525 & knear & srby87 & 3.5 & 5.1 & 0.40 & 3.2 & 4.8 & 0.34 & 3.3 & 5.3 & 0.36 \\
\hline 9.30 & 0.70 & 15.00 & 2.00 & 2.80 & 0.122 & knear & srby 87 & 2.6 & 6.0 & 0.11 & 2.4 & 5.6 & 0.09 & 2.4 & 6.1 & 0.09 \\
\hline 9.30 & 0.00 & 31.00 & 15.60 & 4.80 & 0.952 & knear & srby 87 & 4.1 & 4.5 & 0.69 & 3.8 & 4.3 & 0.60 & 3.9 & 4.7 & 0.63 \\
\hline 9.60 & 0.80 & 26.00 & 53.90 & 4.20 & 3.288 & knear & srby 87 & 3.6 & 4.9 & 2.42 & 3.4 & 4.7 & 2.15 & 3.4 & 5.1 & 2.15 \\
\hline 9.80 & -0.75 & 28.00 & 18.30 & 4.40 & 1.116 & knear & y 87 & 3.8 & 4.8 & 0.83 & 3.5 & 4.6 & 0.71 & 3.6 & 5.0 & 0.75 \\
\hline 10.00 & -0.04 & 32.00 & 18.30 & 4.70 & 1.116 & knear & y87 & 4.0 & 4.6 & 0.81 & 3.7 & 4.4 & 0.69 & 3.8 & 4.8 & 0.73 \\
\hline 10.80 & -0.80 & 29.00 & 38.00 & 4.20 & 2.318 & knear & y87 & 3.7 & 5.0 & 1.80 & 3.4 & 4.7 & 1.52 & .5 & 5.1 & 1.61 \\
\hline 11.10 & -0.50 & 34.00 & 78.00 & 4.80 & 35.258 & kfar & y87 & 12.7 & 4.7 & 246.82 & 12.0 & 4.4 & 220.36 & 12.8 & 4.7 & 50.72 \\
\hline 11.40 & -0.30 & 49.00 & 17.90 & 6.10 & 1.092 & knear & y 87 & 4.8 & 3.9 & 0.68 & 4.4 & 3.8 & 0.57 & 4.5 & 4.2 & 0.59 \\
\hline 11.60 & -0.40 & 32.00 & 16.70 & 4.30 & 019 & knear & srby 87 & 3.7 & 4.9 & 0.75 & 3.5 & 4.6 & 0.68 & 3.5 & 5.1 & 0.68 \\
\hline .70 & -0.30 & 47.00 & 12.30 & 5.60 & 0.750 & knear & srby 87 & 4.6 & 4.1 & 0.51 & 4.3 & 3.9 & 0.44 & 4.3 & 4.3 & 0.44 \\
\hline .90 & 0.80 & 26.00 & 22.40 & 3.60 & 1.366 & knear & srby87 & 3.2 & 5.4 & 1.08 & 3.0 & 5.1 & 0.95 & 3.0 & 5.6 & 0.95 \\
\hline .90 & -0.10 & 42.00 & 84.60 & 14.40 & 5.161 & kfar & srby87 & 12.3 & 4.4 & 3.77 & 11.6 & 4.2 & 3.35 & 12.4 & 4.4 & 3.83 \\
\hline 12.00 & -0.60 & 35.00 & 29.50 & 4.50 & 1.799 & knear & srby 87 & 3.9 & 4.8 & 1.35 & 3.6 & 4.5 & 1.15 & 3.7 & 5.0 & 1.22 \\
\hline 12.20 & 0.10 & 23.00 & 83.00 & 16.30 & 5.063 & kfar & srby87 & 13.7 & 5.7 & 3.58 & 12.9 & 5.4 & 3.17 & 13.6 & 5.6 & 3.52 \\
\hline 12.40 & -0.70 & 25.00 & 3.20 & 3.40 & 0.195 & knear & srby87 & 3.1 & 5.5 & 0.16 & 2.9 & 5.2 & 0.14 & 2.9 & .7 & 0.14 \\
\hline 12.70 & 0.70 & 18.00 & 28.10 & 2.50 & 1.714 & knear & srby 87 & 2.4 & 6.2 & 1.58 & 2.2 & 5.8 & 1.33 & 2.3 & 6.3 & 1.45 \\
\hline 2.70 & -0.10 & 56.00 & 45.70 & 13.60 & 2.788 & kfar & srby 87 & 11.7 & 3.9 & 2.06 & 11.1 & 3.7 & 1.86 & 11.8 & .0 & 2.10 \\
\hline 12.80 & -0.20 & 32.00 & 278.00 & 4.10 & 16.958 & knear & srby87 & 3.5 & 5.1 & 12.36 & 3.3 & 4.8 & 10.99 & 3.3 & .3 & 10.99 \\
\hline 12.90 & 0.50 & 0 & & 4.30 & 640 & ear & & 3.7 & 4.9 & 47 & 3.5 & 4.7 & 0.42 & 3.5 & .1 & 0.42 \\
\hline 13.00 & 0.40 & .00 & & 4.00 & 153 & ear & & 3.5 & 5.1 & 65 & 3.3 & 4.9 & .47 & 3.3 & 3 & 1.47 \\
\hline 13.00 & 0.10 & 4 & 40.00 & & & ear & & 4.4 & 4.3 & 89 & 4.1 & 4.1 & .11 & 4.2 & .5 & 5.36 \\
\hline .70 & -0.10 & 4 & & 5. & 928 & hear & & 4.4 & 4.4 & 38 & 4.1 & 4.2 & 1.20 & 4.1 & & 1.20 \\
\hline .90 & 0.90 & 27.00 & & 3.30 & 0.805 & knear & & 3.0 & 5.6 & 0.67 & 2.8 & 5. & 0.58 & 2.9 & & 0.62 \\
\hline .90 & 0.30 & 4 & 47.20 & 5.20 & 2.879 & knear & srby 87 & 4.4 & 4.4 & 2.06 & 4.1 & 4.2 & 1.79 & 4.1 & 4 & 1.79 \\
\hline 14.00 & -0.10 & 26.00 & 7.60 & 3.20 & 0.464 & knear & srby87 & 2.9 & 5.7 & 0.38 & 2.7 & 5.4 & 0.33 & 2.8 & 8 & 0.36 \\
\hline 14.00 & -0.60 & 18.00 & 195.00 & 2.40 & 11.895 & knear & srby87 & 2.2 & 6.4 & 0.00 & 2.1 & 6.0 & 9.11 & 2.2 & 6.4 & 0.00 \\
\hline 14.20 & -0.20 & 39.00 & 97.00 & 4.40 & 18.117 & knear & srby 87 & 3.8 & 4.9 & 3.51 & 3.5 & 4.6 & 11.46 & 3.6 & 5.1 & 12.13 \\
\hline 14.60 & -0.60 & 7.00 & 90 & 4.20 & 1.092 & knear & srby87 & 3.6 & 5.1 & 80 & 3.4 & 4.8 & 0.72 & 3.4 & 5.2 & 0.72 \\
\hline 15.00 & -0.70 & .00 & 5.10 & 2.50 & 531 & knear & y87 & 2.3 & 6.3 & 30 & 2.2 & 5.9 & 1.19 & 2.2 & 0.4 & 1.19 \\
\hline .00 & 0.00 & 2 & .50 & 3.00 & 0.213 & knear & srby 87 & 2.7 & 5.9 & 0.17 & 2.6 & 5.6 & 0.16 & 2.6 & 6.0 & 0.16 \\
\hline & 90 & & & & & ar & & 3.9 & 4.8 & & 3.7 & 4.6 & .19 & 3.7 & .0 & 0.19 \\
\hline 30 & 0.00 & & & & & ear & & 3.2 & 5.5 & 37 & 3.0 & 5.2 & 0.77 & 3.1 & .6 & 0.82 \\
\hline 70 & -0.20 & & & & 305 & ear & & 4.6 & 4.3 & & 4.2 & 4.1 & 0.49 & 4.3 & .5 & 0.51 \\
\hline & .60 & & & & & lear & & 4.1 & 4.7 & & 3.8 & 4.4 & .70 & 3.9 & & 0.74 \\
\hline & .60 & & & & & & & 2.1 & 6.5 & & 2.0 & 6.1 & .81 & 2.1 & & 0.89 \\
\hline & & & & & & & & 2.1 & 6. & & 2.0 & 6.1 & .21 & 2.1 & & 0.23 \\
\hline & 1.00 & & 7.30 & 5.5 & 45 & kne & & 4.6 & 4.3 & & 4.3 & 4.1 & 0.27 & 4.3 & & 0.27 \\
\hline 16.20 & .80 & & 3.20 & & 195 & knear & & 4.5 & 4.4 & & 4.1 & 4.2 & 0.12 & 4.2 & & 0.12 \\
\hline 16.25 & -0.05 & .00 & 143.00 & 14.50 & 723 & kfar & srby87 & 12.3 & 4.7 & 6.28 & 11.6 & 4.5 & 5.58 & 12.3 & 4.8 & 6.28 \\
\hline 16.30 & 0.40 & 2 & .10 & 3.10 & 0.982 & knear & y87 & 2.8 & 5.8 & 0.80 & 2.7 & 5.5 & 0.74 & 2.7 & 6.0 & 0.74 \\
\hline .50 & 0.40 & & 60 & 3.90 & 270 & knear & y87 & 3.5 & 5.3 & 2.63 & 3.2 & 5.0 & 2.20 & 3.3 & 5.4 & 2.34 \\
\hline 16.65 & -0.05 & 5 & 9.80 & 5.40 & 3.038 & knear & srby 87 & 4.6 & 4.3 & 2.20 & 4.2 & 4.1 & 1.84 & 4.3 & 4.6 & 1.93 \\
\hline 16.70 & -0.45 & 43.00 & 66.00 & 4.30 & 4.026 & knear & srby 87 & 3.7 & 5.0 & 2.98 & 3.5 & 4.8 & 2.67 & 3.5 & 5.2 & 2.67 \\
\hline 16.80 & 0.40 & 22.00 & & 2.00 & 6.166 & stel & $\operatorname{dect} 86$ & & & 6.17 & & & 6.17 & & & 6.17 \\
\hline 16.80 & 0.10 & 30.00 & 284.00 & 15.90 & 17.324 & kfar & srby 87 & 13.4 & 5.8 & 12.30 & 12.6 & 5.4 & 10.88 & 13.3 & 5.7 & 12.12 \\
\hline 16.90 & 0.30 & 24.00 & 7.30 & 2.60 & 5.935 & knear & srby87 & 2.5 & 6.2 & 5.49 & 2.3 & 5.8 & 4.64 & 2.4 & 6.3 & 5.06 \\
\hline 17.20 & -0.20 & 43.00 & 910.00 & 14.90 & 55.510 & kfar & srby 87 & 12.5 & 5.1 & 39.07 & 11.8 & 4.8 & 34.81 & 12.6 & 5.1 & 39.70 \\
\hline 17.30 & -0.90 & 4 & & 4.40 & 0.323 & knear & srby 87 & 3.8 & 5.0 & 0.24 & 3.6 & 4.7 & 0.22 & 3.6 & 5.1 & 0.22 \\
\hline 17.70 & 0.30 & 22.00 & 9.90 & 2.40 & 0.604 & knear & srby87 & 2.2 & 6.4 & 0.51 & 2.1 & 6.0 & 0.46 & 2.2 & 6.5 & 0.51 \\
\hline 17.70 & 0.10 & 41.00 & 51.00 & 15.10 & 3.111 & kfar & srby 87 & 12.7 & 5.2 & 2.20 & 11.9 & 5.0 & 1.93 & 12.7 & 5.2 & 2.20 \\
\hline 18.15 & -0.30 & 54.00 & 245.00 & 4.90 & 14.945 & knear & srby 87 & 4.2 & 4.7 & 10.98 & 3.9 & 4.5 & 9.47 & 3.9 & 4.9 & 9.47 \\
\hline 18.65 & 0.30 & 21.00 & 63.50 & 16.80 & 3.873 & kfar & srby 87 & 14.0 & 6.6 & 2.69 & 13.2 & 6.2 & 2.39 & 13.9 & 6.4 & 2.65 \\
\hline
\end{tabular}


Table A.2. continued.

\begin{tabular}{|c|c|c|c|c|c|c|c|c|c|c|c|c|c|c|c|c|}
\hline $\begin{array}{r}l \\
\left({ }^{\circ}\right) \\
(1)\end{array}$ & $\begin{array}{r}b \\
\left({ }^{\circ}\right) \\
(2)\end{array}$ & $\begin{array}{r}V_{\mathrm{lsr}} \\
\mathrm{km} \mathrm{s}^{-1} \\
(3)\end{array}$ & $\begin{array}{r}L_{\mathrm{CO}} \\
10^{3} \mathrm{~K} \mathrm{~km} \mathrm{~s}^{-1} \mathrm{pc}^{2} \\
(4)\end{array}$ & $\begin{array}{r}D \\
\mathrm{kpc} \\
(5)\end{array}$ & $\begin{array}{r}M_{\mathrm{GMCs}} \\
10^{5} M_{\odot} \\
(6)\end{array}$ & Mark & $\overline{\text { Ref. }}$ & $\begin{array}{r}D_{8.5} \\
\mathrm{kpc} \\
(9)\end{array}$ & $\begin{array}{l}R_{8.5} \\
\mathrm{kpc} \\
(10)\end{array}$ & $\begin{array}{r}M_{8.5} \\
10^{5} M_{\odot} \\
(11)\end{array}$ & $\begin{array}{l}D_{8.0} \\
\text { kpc } \\
(12)\end{array}$ & $\begin{array}{l}R_{8.0} \\
\mathrm{kpc} \\
(13)\end{array}$ & $\begin{array}{r}M_{8.0} \\
10^{5} M_{\odot} \\
(14)\end{array}$ & $\begin{array}{l}D_{8.4} \\
\mathrm{kpc} \\
(15)\end{array}$ & $\begin{array}{l}R_{8.4} \\
\mathrm{kpc} \\
(16)\end{array}$ & $\begin{array}{r}M_{8.4} \\
10^{5} M_{\odot} \\
(17)\end{array}$ \\
\hline 18.85 & 0.05 & 50.00 & 330.00 & 14.40 & 20.130 & kfar & srby 87 & 12.2 & 5.0 & 14.45 & 11.5 & 4.7 & 12.84 & 12.2 & 5.0 & 14.45 \\
\hline 18.85 & 0.50 & 66.00 & 576.00 & 5.60 & 35.136 & knear & srby87 & 4.7 & 4.4 & 24.75 & 4.3 & 4.2 & 20.72 & 4.4 & 4.6 & 21.69 \\
\hline 19.20 & 0.30 & 33.00 & 272.00 & 15.70 & 16.592 & kfar & srby 87 & 13.2 & 5.8 & 11.73 & 12.4 & 5.5 & 10.35 & 13.1 & 5.8 & 11.55 \\
\hline 19.25 & 0.05 & 26.00 & 7.70 & 2.60 & 0.470 & knear & srby 87 & 2.4 & 6.3 & 0.40 & 2.3 & 5.9 & 0.37 & 2.3 & 6.3 & 0.37 \\
\hline 19.50 & 0.00 & 26.00 & & 2.20 & 0.891 & stel & $\operatorname{dect} 86$ & & & 0.89 & & & 0.89 & & & 0.89 \\
\hline 19.60 & -0.05 & 58.00 & 13.70 & 4.90 & 0.836 & knear & srby87 & 4.2 & 4.7 & 0.61 & 3.9 & 4.5 & 0.53 & 4.0 & 4.9 & 0.56 \\
\hline 19.65 & -0.65 & 55.00 & 14.60 & 4.80 & 0.891 & knear & srby 87 & 4.1 & 4.8 & 0.65 & 3.8 & 4.6 & 0.56 & 3.9 & 5.0 & 0.59 \\
\hline 19.70 & 0.10 & 26.00 & 218.00 & 16.30 & 13.298 & kfar & srby87 & 13.6 & 6.3 & 9.26 & 12.8 & 5.9 & 8.20 & 13.5 & 6.2 & 9.12 \\
\hline 19.80 & -0.45 & 69.00 & 153.00 & 5.60 & 9.333 & knear & srby87 & 4.7 & 4.4 & 6.57 & 4.4 & 4.2 & 5.76 & 4.4 & 4.6 & 5.76 \\
\hline 19.90 & 0.55 & 44.00 & 111.00 & 4.00 & 6.771 & knear & srby 87 & 3.5 & 5.3 & 5.18 & 3.3 & 5.0 & 4.61 & 3.3 & 5.5 & 4.61 \\
\hline 20.55 & 0.45 & 67.00 & 108.00 & 13.30 & 6.588 & kfar & srby 87 & 11.3 & 4.5 & 4.76 & 10.7 & 4.3 & 4.26 & 11.4 & 4.6 & 4.84 \\
\hline 20.70 & 0.30 & 63.00 & 72.70 & 13.60 & 4.435 & kfar & ру87 & 11.5 & 4.7 & 3.17 & 10.9 & 4.4 & 2.85 & 11.6 & 4.7 & 3.23 \\
\hline 20.75 & 0.10 & 59.00 & 168.00 & 13.80 & 10.248 & kfar & ру87 & 11.7 & 4.8 & 7.37 & 11.0 & 4.6 & 6.51 & 11.8 & 4.8 & 7.49 \\
\hline 20.75 & 10 & 77.00 & 31.60 & 6.00 & 1.928 & knear & by 87 & 5.0 & 4.2 & 1.34 & 4.6 & 4.0 & 1.13 & 4.7 & 4.5 & 1.18 \\
\hline 20.85 & 00 & 30.00 & 262.00 & 15.90 & 5.982 & kfar & srby87 & 13.3 & 6.2 & 1.18 & 12.5 & 5.8 & 9.88 & 13.2 & 6.1 & 11.02 \\
\hline 20.90 & .30 & 3.00 & 31.60 & 5.40 & 928 & knear & srby 87 & 4.6 & 4.5 & 1.40 & 4.3 & 4.3 & 1.22 & 4.3 & 4.7 & 1.22 \\
\hline 21.35 & 35 & 8.00 & 3.00 & 0.80 & 0.183 & knear & srby 87 & 0.7 & 7.8 & 0.14 & 0.8 & 7.3 & 0.18 & 0.9 & 7.7 & 0.23 \\
\hline 21.40 & -0.65 & 55.00 & 87.50 & 4.60 & 5.337 & knear & srby 87 & 4.0 & 5.0 & 4.04 & 3.7 & 4.7 & 3.45 & 3.8 & 5.2 & 3.64 \\
\hline 21.40 & 0.00 & 75.00 & 153.00 & 12.80 & 9.333 & kfar & srby 87 & 11.0 & 4.3 & 6.89 & 10.4 & 4.1 & 6.16 & 11.1 & 4.4 & 7.02 \\
\hline 21.50 & 0.25 & 77.00 & 5.50 & 5.90 & 0.335 & knear & srby 87 & 5.0 & 4.3 & 0.24 & 4.6 & 4.1 & 0.20 & 4.6 & 4.5 & 0.20 \\
\hline 21.75 & 0.00 & 67.00 & 46.20 & 5.30 & 2.818 & knear & srby 87 & 4.5 & 4.6 & 2.03 & 4.2 & 4.4 & 1.77 & 4.2 & 4.8 & 1.77 \\
\hline 21.90 & -0.35 & 82.00 & 409.00 & 12.40 & 24.949 & kfar & srby87 & 10.6 & 4.2 & 18.23 & 10.1 & 4.0 & 16.55 & 10.8 & 4.3 & 18.93 \\
\hline 22.05 & 0.20 & 50.00 & 70.90 & 4.20 & 4.325 & knear & srby 87 & 3.7 & 5.3 & 3.36 & 3.4 & 5.0 & 2.83 & 3.5 & 5.4 & 3.00 \\
\hline 22.10 & -0.20 & 53.00 & & 4.40 & 11.482 & knear & $\operatorname{dect} 86$ & 3.8 & 5.2 & 8.56 & 3.6 & 4.9 & 7.69 & 3.6 & 5.3 & 7.69 \\
\hline 2.35 & 0.10 & 5.00 & 71.70 & 12.20 & 4.374 & kfar & y87 & 10.5 & 4.2 & 3.24 & 10.0 & 4.0 & 2.94 & 10.6 & 4.3 & 3.30 \\
\hline 22.40 & 30 & .00 & 53.00 & 12.20 & 3 & kfar & by 87 & 10.5 & 4.2 & 2.39 & 10.0 & 4.0 & 2.17 & 10.7 & 4.3 & 2.49 \\
\hline 22.55 & -0.05 & 115.00 & 23.20 & 8.50 & 415 & ktan & by 87 & & & & & & & & & \\
\hline 22.55 & .20 & .00 & 4.80 & 12.60 & & kfar & & 10.8 & 4.4 & 2.90 & 10.2 & 4.2 & 2.59 & 10.9 & 4.5 & 2.96 \\
\hline 22.75 & .25 & 9.00 & 40.30 & 9.20 & 8 & ktan & & & & & & & & & & \\
\hline 22.80 & .40 & .00 & 90.50 & 11.70 & .520 & kfar & srby 87 & 10.2 & 4.0 & 4.20 & 9.7 & 3.9 & 3.79 & 10.4 & 4.1 & 4.36 \\
\hline 22.85 & 0.40 & 4.00 & 8.10 & 8.40 & 0.494 & ktan & & & & & & & & & & \\
\hline 23.00 & 0.10 & 3.00 & & 5.90 & 11.220 & knear & $\operatorname{dect} 86$ & 4.9 & 4.4 & 7.74 & 4.6 & 4.2 & 6.82 & 4.6 & 4.6 & 6.82 \\
\hline 23.00 & .10 & 8.00 & & 12.60 & 51.286 & kfar & $\operatorname{dect} 86$ & 10.7 & 4.4 & 36.98 & 10.2 & 4.2 & 33.61 & 10.9 & 4.5 & 38.38 \\
\hline 23.00 & -0.40 & 1.00 & 2901.00 & 12.80 & 176.961 & kfar & srby 87 & 10.9 & 4.5 & 128.32 & 10.3 & 4.3 & 114.59 & 11.0 & 4.6 & 130.69 \\
\hline 23.00 & 00 & .00 & 202.00 & 12.40 & .322 & kfar & by87 & 10.6 & 4.3 & 9.00 & 10.1 & 4.1 & 8.17 & 10.8 & 4.4 & 9.35 \\
\hline 23.10 & 60 & .00 & 3.30 & 3.20 & 201 & knear & y87 & 2.9 & 5.9 & 0.17 & 2.8 & 5.6 & 0.15 & 2.8 & 6.0 & 0.15 \\
\hline 23.30 & 0.30 & .00 & & 4.00 & 450 & knear & w07 & 4.3 & 4.9 & 0.52 & 4.0 & 4.6 & 0.45 & 4.0 & 5.0 & 0.45 \\
\hline .40 & .25 & 60 & 258.00 & 11.00 & & $\mathrm{ktan}$ & & & & & & & & & & \\
\hline 50 & 40 & & 21.80 & 50 & & knear & & 4.7 & 4.6 & 0.97 & 4.3 & 4.4 & 0.81 & 4.4 & 4.8 & 0.85 \\
\hline 55 & & & 280.00 & 12.30 & & kfar & & 10.5 & 4.3 & 12.45 & 10.0 & 4.1 & .29 & 10.7 & 4.4 & 12.93 \\
\hline 70 & & & 9.70 & 6.10 & & knear & & 5.1 & 4.4 & .41 & 4.7 & 4.2 & 35 & 4.7 & 4.6 & 0.35 \\
\hline & & & & 7.10 & & ear & & 5.8 & 4.0 & .25 & 5.3 & 3.8 & 07 & 5.3 & 4.2 & 16.07 \\
\hline & & & .00 & 5.80 & & knear & & 4.9 & 4.5 & 0.65 & 4.6 & 4.2 & 58 & 4.6 & 4.7 & 0.58 \\
\hline 1.20 & .05 & & .70 & 11.90 & & kfar & & 10.2 & 4.2 & 4.07 & 9.7 & 4.1 & 3.68 & 10.4 & 4.4 & 4.23 \\
\hline 40 & .25 & & 111.00 & 13.60 & 771 & kfar & & 11.4 & 5.1 & 4.76 & 10.8 & 4.8 & 4.27 & 11.5 & 5.1 & 4.84 \\
\hline 45 & -0.80 & 0 & 4.80 & 4.30 & 293 & knear & srby87 & 3.9 & 5.2 & 0.24 & 3.6 & 5.0 & 0.21 & 3.7 & 5.4 & 0.22 \\
\hline 45 & 25 & 0.00 & 1095.00 & 9.10 & 66.795 & $\mathrm{ktan}$ & & & & & & & & & & \\
\hline 24.45 & -0.50 & .00 & 3.50 & 3.50 & 6.008 & knear & srby 87 & 3.2 & 5.7 & 5.02 & 3.0 & & 4.41 & 3.1 & 5.8 & 4.71 \\
\hline 24.50 & & 3 & 8.20 & 15.10 & 770 & kfar & srby 87 & 12.6 & 6.0 & 3.32 & 11.9 & 5.7 & 2.96 & 12.5 & 5.9 & 3.27 \\
\hline 24.50 & 0.15 & 0 & 1.50 & 3.50 & 311 & knear & srby87 & 3.2 & 5.7 & 1.10 & 3.0 & 5.4 & 0.96 & 3.1 & 5.8 & 1.03 \\
\hline 24.50 & 0.70 & .00 & .80 & 3.70 & 720 & knear & srby 87 & 3.4 & 5.6 & 0.61 & 3.2 & 5.3 & 0.54 & 3.2 & 5.7 & 0.54 \\
\hline 24.50 & 0.25 & 0 & 7.00 & 10.90 & & kfar & & 9.6 & 4.0 & & 9.1 & 3.8 & 6.68 & 9.9 & 4.1 & 7.90 \\
\hline 24.55 & .50 & & .00 & & 2.135 & knear & & 4.1 & 5.1 & 1.56 & 3.8 & 4.8 & 1.34 & 3.9 & 5.2 & 1.41 \\
\hline 24.60 & .15 & 3.00 & 212.00 & 12.10 & 12.932 & kfar & srby 87 & 10.4 & 4.4 & 9.55 & 9.8 & 4.2 & 8.48 & 10.5 & 4.5 & 9.74 \\
\hline & -0.10 & & & & & ktan & & & & & & & & & & \\
\hline & & & & & & ktan & & & & & & & & & & \\
\hline & & & 161.00 & & & kfar & & 12.0 & & & & & 6.05 & & 5.6 & 6.82 \\
\hline & 0.50 & & & 13.90 & & kfar & & 11.6 & 5.3 & & 10.9 & 5.0 & 29.43 & 11.6 & 5.4 & 3.33 \\
\hline & .20 & & 620.00 & 13.20 & & kfar & srby 87 & 11.1 & 5.0 & 26.74 & 10.5 & 4.7 & 23.93 & 11.2 & 5.1 & 27.23 \\
\hline & .20 & & & & & ktan & & & & & & & & & & \\
\hline & .40 & & & & & ktan & & & & & & & & & & \\
\hline & & & & 11.50 & 48.373 & kfar & & 9.7 & 4.2 & 34.42 & 9.3 & 4.0 & 31.64 & 10.0 & 4.3 & 36.58 \\
\hline & .15 & & & 9. & & & & & & & & & & & & \\
\hline 25.80 & 0.45 & 48.00 & 34.70 & 3.70 & 2.117 & knear & srby 87 & 3.4 & 5.7 & 1.79 & 3.2 & 5.3 & 1.58 & 3.2 & 5.8 & 1.58 \\
\hline
\end{tabular}


Table A.2. continued.

\begin{tabular}{|c|c|c|c|c|c|c|c|c|c|c|c|c|c|c|c|c|}
\hline $\begin{array}{r}{ }^{l} \\
\left({ }^{\circ}\right) \\
(1)\end{array}$ & $\begin{array}{l}b \\
\left({ }^{\circ}\right) \\
(2)\end{array}$ & $\begin{array}{r}V_{\mathrm{lsr}} \\
\mathrm{km} \mathrm{s}^{-1} \\
\text { (3) }\end{array}$ & $\begin{array}{r}L_{\mathrm{CO}} \\
10^{3} \mathrm{~K} \mathrm{~km} \mathrm{~s}^{-1} \mathrm{pc}^{2} \\
(4)\end{array}$ & $\begin{array}{r}D \\
\mathrm{kpc} \\
(5)\end{array}$ & $\begin{array}{r}M_{\mathrm{GMCs}} \\
10^{5} M_{\odot} \\
(6)\end{array}$ & Mark & Ref. & $\begin{array}{r}D_{8.5} \\
\mathrm{kpc} \\
(9)\end{array}$ & $\begin{array}{l}R_{8.5} \\
\mathrm{kpc} \\
(10)\end{array}$ & $\begin{array}{r}M_{8.5} \\
10^{5} M_{\odot} \\
(11)\end{array}$ & $\begin{array}{l}D_{8.0} \\
\mathrm{kpc} \\
(12)\end{array}$ & $\begin{array}{l}R_{8.0} \\
\mathrm{kpc} \\
(13)\end{array}$ & $\begin{array}{r}M_{8.0} \\
10^{5} M_{\odot} \\
(14)\end{array}$ & $\begin{array}{l}D_{8.4} \\
\mathrm{kpc} \\
(15)\end{array}$ & $\begin{array}{l}R_{8.4} \\
\mathrm{kpc} \\
(16)\end{array}$ & $\begin{array}{r}M_{8.4} \\
10^{5} M_{\odot} \\
(17)\end{array}$ \\
\hline 25.80 & 0.25 & 109.00 & 347.00 & 9.00 & 21.167 & ktan & srby87 & & & & & & & & & \\
\hline 25.90 & 0.20 & 69.00 & 4.50 & 5.10 & 0.274 & knear & srby87 & 4.4 & 4.9 & 0.20 & 4.1 & 4.7 & 0.18 & 4.2 & 5.1 & 0.19 \\
\hline 25.90 & -0.15 & 107.00 & 273.00 & 9.00 & 16.653 & ktan & srby87 & & & & & & & & & \\
\hline 25.95 & -0.60 & 63.00 & 9.40 & 4.70 & 0.573 & knear & srby 87 & 4.1 & 5.1 & 0.44 & 3.9 & 4.8 & 0.39 & 3.9 & 5.3 & 0.39 \\
\hline 26.20 & 0.10 & 71.00 & 17.30 & 5.20 & 1.055 & knear & srby 87 & 4.5 & 4.9 & 0.79 & 4.2 & 4.6 & 0.69 & 4.2 & 5.1 & 0.69 \\
\hline 26.35 & 0.80 & 47.00 & 5.30 & 3.60 & 0.323 & knear & srby87 & 3.3 & 5.7 & 0.27 & 3.1 & 5.4 & 0.24 & 3.2 & 5.8 & 0.26 \\
\hline 26.50 & -0.60 & 67.00 & 93.50 & 4.90 & 5.703 & knear & srby 87 & 4.3 & 5.0 & 4.39 & 4.0 & 4.8 & 3.80 & 4.1 & 5.2 & 3.99 \\
\hline 26.55 & -0.30 & 108.00 & 39.50 & 8.90 & 2.409 & ktan & srby87 & & & & & & & & & \\
\hline 26.60 & 0.00 & 26.00 & 186.00 & 15.80 & 11.346 & kfar & srby87 & 13.2 & 6.8 & 7.92 & 12.4 & 6.3 & 6.99 & 13.0 & 6.6 & 7.68 \\
\hline 26.65 & 0.00 & 100.00 & 222.00 & 9.60 & 13.542 & ktan & srby 87 & & & & & & & & & \\
\hline 26.65 & 0.00 & 2.00 & 23.90 & 8.90 & 1.458 & ktan & rby 87 & & & & & & & & & \\
\hline 26.70 & 0.50 & 6.00 & 13.40 & 6.20 & 0.817 & knear & srby87 & 5.2 & 4.5 & 0.57 & 4.8 & 4.3 & 0.49 & 4.8 & 4.7 & 0.49 \\
\hline 26.90 & 0.10 & 91.00 & 44.40 & 6.50 & 2.708 & knear & rby 87 & 5.5 & 4.4 & 1.94 & 5.0 & 4.2 & 1.60 & 5.1 & 4.6 & 1.67 \\
\hline 26.95 & -0.40 & 69.00 & 5.70 & 5.00 & 0.348 & knear & rby87 & 4.4 & 5.0 & 0.27 & 4.1 & 4.7 & 0.23 & 4.1 & 5.2 & 0.23 \\
\hline 26.95 & -0.10 & 81.00 & 22.10 & 5.80 & 1.348 & knear & srby87 & 5.0 & 4.6 & 1.00 & 4.6 & 4.4 & 0.85 & 4.6 & 4.8 & 0.85 \\
\hline 27.05 & -0.15 & 101.00 & 21.50 & 8.90 & 1.311 & ktan & srby 87 & & & & & & & & & \\
\hline 27.25 & 0.15 & 33.00 & 148.00 & 15.20 & 9.028 & kfar & srby 87 & 12.7 & 6.4 & 6.30 & 11.9 & 6.0 & 5.53 & 12.5 & 6.3 & 6.11 \\
\hline 27.30 & -0.30 & 72.00 & 14.50 & 5.20 & 0.884 & knear & srby 87 & 4.5 & 4.9 & 0.66 & 4.2 & 4.7 & 0.58 & 4.3 & 5.1 & 0.60 \\
\hline 27.35 & -0.15 & 93.00 & 18.00 & 6.60 & 1.098 & knear & srby 87 & 5.6 & 4.4 & 0.79 & 5.1 & 4.2 & 0.66 & 5.1 & 4.6 & 0.66 \\
\hline 27.50 & 0.20 & 36.00 & 46.10 & 15.00 & 2.812 & kfar & srby87 & 12.5 & 6.3 & 1.95 & 11.7 & 5.9 & 1.71 & 12.4 & 5.2 & 1.92 \\
\hline 27.50 & 0.15 & 95.00 & 167.00 & 10.90 & 10.187 & kfar & srby87 & 9.4 & 4.3 & 7.58 & 9.0 & 4.1 & 6.95 & 9.7 & .5 & 8.07 \\
\hline 7.65 & 0.10 & 83.00 & 32.10 & 5.90 & 1.958 & knear & srby87 & 5.1 & 4.6 & 1.46 & 4.7 & 4.4 & 1.24 & 4.7 & 4.8 & 1.24 \\
\hline 27.75 & 0.10 & 101.00 & 141.00 & 8.90 & 8.601 & ktan & srby 87 & & & & & & & & & \\
\hline 28.20 & -0.05 & 5.00 & 150.00 & 10.80 & 9.150 & kfar & srby 87 & 9.3 & 4.4 & 6.78 & 8.8 & 4.2 & 6.07 & 9.6 & .5 & 7.23 \\
\hline 28.30 & -0.35 & 47.00 & 18.60 & 3.50 & 1.135 & knear & srby 87 & 3.2 & 5.9 & 0.95 & 3.0 & 5.5 & 0.83 & 3.1 & .0 & 0.89 \\
\hline 28.30 & -0.10 & 1.00 & 36.00 & 5.80 & 11.346 & knear & srby 87 & 5.0 & 4.7 & 8.43 & 4.6 & 4.5 & 7.14 & 4.6 & .9 & 7.14 \\
\hline 28.60 & 0.05 & 100.00 & 141.00 & 8.80 & 601 & ktan & srby 87 & & & & & & & & & \\
\hline 28.60 & 0.20 & 79.00 & 68.10 & 11.90 & 4.154 & kfar & srby 87 & 10.0 & 4.8 & 2.93 & 9.5 & 4.6 & 2.65 & 10.2 & 4.9 & 3.05 \\
\hline 28.80 & -0.10 & 80.00 & & 5.70 & 21.380 & knear & $\operatorname{dect86}$ & 4.9 & 4.8 & 15.80 & 4.6 & 4.6 & 13.92 & 4.6 & 5.0 & 13.92 \\
\hline 28.85 & -0.25 & 88.00 & 94.40 & 6.30 & 5.758 & knear & srby 87 & 5.4 & 4.6 & 4.23 & 4.9 & 4.4 & 3.48 & 4.9 & 4.8 & 3.48 \\
\hline 28.95 & -0.65 & 51.00 & 7.60 & 3.80 & 0.464 & knear & srby 87 & 3.4 & 5.7 & 0.37 & 3.2 & 5.4 & 0.33 & 3.3 & 5.8 & 0.35 \\
\hline 29.00 & 0.05 & 98.00 & 255.00 & 8.80 & 15.555 & ktan & srby 87 & & & & & & & & & \\
\hline 29.10 & -0.30 & 4.00 & 223.00 & 7.10 & 13.603 & knear & srby 87 & 5.7 & 4.5 & 8.77 & 5.2 & 4.3 & 7.30 & 5.2 & 4.7 & 7.30 \\
\hline 29.35 & -0.45 & 77.00 & 141.00 & 5.40 & 8.601 & knear & srby 87 & 4.8 & 4.9 & 6.80 & 4.4 & 4.7 & 5.71 & 4.5 & 5.1 & 5.97 \\
\hline 29.35 & -0.55 & 1.00 & 25.70 & 4.60 & .568 & knear & 87 & 4.1 & 5.3 & 1.25 & 3.8 & 5.0 & 1.07 & 3.9 & 5.5 & 1.13 \\
\hline .55 & 0.20 & 9.00 & 87.40 & 5.60 & 5.331 & knear & srby87 & 4.9 & 4.9 & 4.08 & 4.5 & 4.6 & 3.44 & 4.6 & .1 & 3.60 \\
\hline 29.60 & -0.60 & .00 & 274.00 & 12.10 & 16.714 & kfar & srby87 & 10.1 & 5.0 & 11.65 & 9.6 & 4.7 & 10.52 & 10.2 & 5.1 & 11.88 \\
\hline 9.85 & -0.05 & 0.00 & 0.00 & 8.70 & 21.350 & ktan & srby87 & & & & & & & & & \\
\hline 29.90 & 0.10 & .00 & .00 & 14.40 & 84 & kfar & & 12.0 & 6.2 & 86 & 11.2 & 5.9 & 1.62 & 11.9 & .2 & 1.83 \\
\hline 29.90 & .80 & 0 & .50 & 5.90 & 067 & knear & & 5.1 & 4.8 & 0.80 & 4.7 & 4.6 & 0.68 & 4.8 & 5.0 & 0.71 \\
\hline 0.30 & -0.25 & 105.00 & 265.00 & 8.60 & 16.165 & ktan & y87 & & & & & & & & & \\
\hline 30.40 & 0.45 & .00 & 2.00 & 3.30 & 0.122 & knear & srby 87 & 3.1 & 6.1 & 0.11 & 2.9 & 5.7 & 0.09 & 3.0 & 6.1 & 0.10 \\
\hline 30.55 & 0.35 & 5.00 & 79.40 & 8.60 & 843 & ktan & srby 87 & & & & & & & & & \\
\hline 30.60 & -0.05 & 43.00 & 321.00 & 14.10 & 19.581 & kfar & srby 87 & 11.7 & 6.2 & 13.48 & 11.0 & 5.8 & 11.92 & 11.6 & 6.1 & 13.25 \\
\hline 30.60 & -0.45 & 4.00 & 75.70 & 10.30 & 4.618 & kfar & srby 87 & 8.8 & 4.6 & 3.37 & 8.4 & 4.4 & 3.07 & 9.1 & 4.7 & 3.60 \\
\hline 30.60 & -0.10 & 115.00 & 56.90 & 8.60 & 3.471 & ktan & srby 87 & 7.3 & 4.3 & 2.50 & 6.9 & 4.1 & 2.23 & & & \\
\hline 30.80 & -0.05 & 00 & 766.00 & 6.80 & 46.726 & knear & srby 87 & 5.7 & 4.6 & 32.83 & 5.2 & 4.4 & 27.32 & 5.2 & 4.8 & 27.32 \\
\hline 30.85 & -0.15 & 3.00 & 8.20 & 3.90 & 0.500 & knear & srby87 & 3.5 & 5.8 & 0.40 & 3.3 & 5.4 & 0.36 & 3.3 & 5.9 & 0.36 \\
\hline 30.90 & -0.60 & 02.00 & 13.10 & 8.60 & 0.799 & ktan & srby 87 & & & & & & & & & \\
\hline 30.95 & 0.10 & .00 & 237.00 & 14.20 & 14.457 & kfar & srby 87 & 11.8 & 6.3 & 9.98 & 11.1 & 5.9 & 8.83 & 11.7 & 6.2 & 9.81 \\
\hline 30.95 & 0.40 & 77.00 & 29.70 & 5.50 & 1.812 & knear & & 4.8 & 5.0 & 1.38 & 4.5 & 4.8 & 1.21 & 4.5 & 5.2 & 1.21 \\
\hline 31.00 & 0.00 & 3.00 & & 13.70 & 28.184 & kfar & & 11.4 & 6.0 & 19.52 & 10.7 & 5.6 & 17.19 & 11.3 & 5.9 & 19.17 \\
\hline 31.30 & 0.00 & & 41.90 & 5.60 & & knear & & 4.9 & 5.0 & & 4.6 & 4. & 1.72 & 4.6 & & 1.72 \\
\hline 1.40 & 0.00 & 3 & 514.00 & 14.20 & 31.354 & kfar & & 11.8 & 6.4 & 21.65 & 11.1 & 6.0 & 19.16 & 11.7 & 6.3 & 21.29 \\
\hline 31.40 & -0.25 & 88.00 & 30.20 & 10.70 & & kfar & & 9.0 & 4.8 & 1.30 & 8.6 & 4.5 & 1.19 & 9.3 & 4.9 & 1.39 \\
\hline 31.45 & 0.05 & 104.00 & 102.00 & & 6.222 & ktan & srby 87 & 7.3 & 4.4 & 4.59 & & & & & & \\
\hline 31.95 & -0.30 & & 49.30 & & 3.007 & ktan & srby 87 & & & & & & & & & \\
\hline .00 & 0.0 & & 166.00 & & 10.126 & ktan & & & & & & & & & & \\
\hline 32.45 & 0.2 & & 34.30 & 13.30 & 092 & kfar & srby87 & 11.0 & 5. & & 10.3 & 5. & 1.25 & 10.9 & 5.9 & 1.41 \\
\hline 32.60 & -0.25 & 0.00 & 565.00 & 10.30 & 34.465 & kfar & srby 87 & 8.6 & 4.8 & 24.03 & 8.2 & 4.6 & 21.84 & 8.9 & 4.9 & 25.73 \\
\hline 33.35 & -0.55 & 2.00 & 36.50 & 8.40 & 226 & ktan & & & & & & & & & & \\
\hline 33.40 & 0.00 & 5.00 & 167.00 & 11.40 & 10.187 & kfar & srby 87 & 9.4 & 5.2 & 6.93 & 8.9 & 4.9 & 6.21 & 9.6 & 5.3 & 7.22 \\
\hline 33.45 & -0.10 & 87.00 & 191.00 & 10.30 & 11.651 & kfar & srby 87 & 8.6 & 4.9 & 8.12 & 8.2 & 4.7 & 7.38 & 8.9 & 5.0 & 8.70 \\
\hline
\end{tabular}


Table A.2. continued.

\begin{tabular}{|c|c|c|c|c|c|c|c|c|c|c|c|c|c|c|c|c|}
\hline $\begin{array}{r}l \\
\left({ }^{\circ}\right) \\
(1)\end{array}$ & $\begin{array}{r}b \\
\left({ }^{\circ}\right) \\
(2)\end{array}$ & $\begin{array}{r}V_{\mathrm{lsr}} \\
\mathrm{km} \mathrm{s}^{-1} \\
\text { (3) }\end{array}$ & $\begin{array}{r}L_{\mathrm{CO}} \\
10^{3} \mathrm{~K} \mathrm{~km} \mathrm{~s}^{-1} \mathrm{pc}^{2} \\
(4)\end{array}$ & $\begin{array}{r}D \\
\mathrm{kpc} \\
(5)\end{array}$ & $\begin{array}{r}M_{\mathrm{GMCS}} \\
10^{5} M_{\odot} \\
(6)\end{array}$ & Mark & Ref. & $\begin{array}{r}D_{8.5} \\
\mathrm{kpc} \\
(9)\end{array}$ & $\begin{array}{l}R_{8.5} \\
\mathrm{kpc} \\
(10)\end{array}$ & $\begin{array}{r}M_{8.5} \\
10^{5} M_{\odot} \\
(11)\end{array}$ & $\begin{array}{l}D_{8.0} \\
\mathrm{kpc} \\
(12)\end{array}$ & $\begin{array}{l}R_{8.0} \\
\mathrm{kpc} \\
(13)\end{array}$ & $\begin{array}{r}M_{8.0} \\
10^{5} M_{\odot} \\
(14)\end{array}$ & $\begin{array}{l}D_{8.4} \\
\mathrm{kpc} \\
(15)\end{array}$ & $\begin{array}{l}R_{8.4} \\
\mathrm{kpc} \\
(16)\end{array}$ & $\begin{array}{r}M_{8.4} \\
10^{5} M_{\odot} \\
(17)\end{array}$ \\
\hline 33.65 & 0.20 & 42.00 & 48.90 & 13.60 & 2.983 & kfar & srby87 & 11.3 & 6.3 & 2.06 & 10.6 & 6.0 & 1.81 & 11.2 & 6.3 & 2.02 \\
\hline 33.80 & -0.20 & 48.00 & 138.00 & 13.20 & 8.418 & kfar & srby87 & 10.9 & 6.1 & 5.74 & 10.3 & 5.7 & 5.13 & 10.9 & 6.1 & 5.74 \\
\hline 33.85 & 0.00 & 89.00 & 57.30 & 10.00 & 3.495 & kfar & srby 87 & 8.3 & 4.9 & 2.41 & 7.9 & 4.6 & 2.18 & 8.7 & 5.0 & 2.65 \\
\hline 33.90 & 0.10 & 106.00 & 182.00 & 8.30 & 11.102 & ktan & srby87 & 7.1 & 4.7 & 8.12 & 6.6 & 4.5 & 7.02 & 7.0 & 4.7 & 7.90 \\
\hline 34.15 & -0.10 & 89.00 & 40.40 & 8.30 & 2.464 & ktan & srby87 & & & & & & & & & \\
\hline 34.25 & 0.10 & 53.00 & 50.90 & 3.70 & 3.105 & knear & srby 87 & 3.5 & 6.0 & 2.78 & 3.3 & 5.6 & 2.47 & 3.3 & 6.0 & 2.47 \\
\hline 34.35 & -0.85 & 13.00 & 2.50 & 0.90 & 0.152 & knear & srby87 & 0.9 & 7.8 & 0.15 & 0.9 & 7.2 & 0.15 & 1.1 & 7.6 & 0.23 \\
\hline 34.40 & -0.20 & 53.00 & 6.70 & 3.70 & 0.409 & knear & srby87 & 3.5 & 6.0 & 0.37 & 3.3 & 5.6 & 0.33 & 3.3 & 6.0 & 0.33 \\
\hline 34.65 & -0.80 & 55.00 & 37.50 & 3.90 & 2.287 & knear & srby 87 & 3.6 & 5.9 & 1.95 & 3.4 & 5.6 & 1.74 & 3.5 & 6.0 & 1.84 \\
\hline 34.70 & -0.70 & 46.00 & 230.00 & 3.30 & 14.030 & knear & srby 87 & 3.1 & 6.2 & 12.38 & 2.9 & 5.9 & 10.83 & 3.0 & 6.3 & 11.60 \\
\hline 34.80 & -0.15 & 76.00 & 38.90 & 5.60 & 2.373 & knear & srby87 & 4.9 & 5.3 & 1.82 & 4.6 & 5.0 & 1.60 & 4.6 & 5.4 & 1.60 \\
\hline 35.05 & 0.30 & 51.00 & 44.10 & 3.60 & 2.690 & knear & srby 87 & 3.4 & 6.1 & 2.40 & 3.2 & 5.7 & 2.13 & 3.2 & 6.1 & 2.13 \\
\hline 35.15 & -0.75 & 35.00 & 30.80 & 3.30 & 1.879 & knear & srby87 & 2.4 & 6.7 & 0.99 & 2.3 & 6.3 & 0.91 & 2.4 & 6.7 & 0.99 \\
\hline 35.20 & -0.10 & 13.00 & 3.80 & 0.90 & 0.232 & knear & srby 87 & 0.9 & 7.8 & 0.23 & 0.9 & 7.3 & 0.23 & 1.1 & 7.6 & 0.35 \\
\hline 35.75 & 0.15 & 82.00 & 273.00 & 10.20 & 16.653 & kfar & srby87 & 8.3 & 5.2 & 11.03 & 7.9 & 4.9 & 9.99 & 8.6 & 5.3 & 11.84 \\
\hline 35.80 & -0.20 & 29.00 & 7.70 & 2.00 & 0.470 & knear & srby 87 & 2.0 & 7.0 & 0.47 & 1.9 & 6.5 & 0.42 & 2.0 & 6.9 & 0.47 \\
\hline 35.95 & -0.50 & 58.00 & 6.20 & 4.10 & 0.378 & knear & srby 87 & 3.8 & 5.9 & 0.32 & 3.6 & 5.5 & 0.29 & 3.6 & 6.0 & 0.29 \\
\hline 36.10 & 0.65 & 77.00 & 101.00 & 5.60 & 6.161 & knear & srby87 & 5.1 & 5.3 & 5.11 & 4.7 & 5.0 & 4.34 & 4.7 & 5.5 & 4.34 \\
\hline 36.30 & -0.10 & 57.00 & & 12.10 & 42.658 & kfar & $\operatorname{dect} 86$ & 10.0 & 5.9 & 29.14 & 9.4 & 5.6 & 25.74 & 10.0 & 5.9 & 29.14 \\
\hline 36.40 & -0.10 & 52.00 & 60.60 & 3.60 & 3.697 & knear & srby87 & 3.4 & 6.1 & 3.30 & 3.2 & 5.7 & 2.92 & 3.3 & 6.2 & 3.11 \\
\hline 36.50 & -0.15 & 77.00 & 107.00 & 5.90 & 6.527 & knear & srby 87 & 5.1 & 5.3 & 4.88 & 4.8 & 5.0 & 4.32 & 4.8 & 5.5 & 4.32 \\
\hline 36.90 & -0.05 & 80.00 & 36.50 & 5.90 & 2.226 & knear & srby87 & 5.5 & 5.3 & 1.93 & 5.1 & 5.0 & 1.66 & 5.0 & 5.4 & 1.60 \\
\hline 37.35 & 0.25 & 88.00 & 199.00 & 8.60 & 12.139 & ktan & srby87 & 6.8 & 5.2 & 7.59 & 6.4 & 4.9 & 6.72 & & & \\
\hline 37.45 & 0.10 & 41.00 & 4.00 & 3.00 & 0.244 & knear & srby87 & 2.7 & 6.5 & 0.20 & 2.6 & 6.1 & 0.18 & 2.7 & 6.6 & 0.20 \\
\hline 37.75 & -0.20 & 60.00 & 137.00 & 11.60 & 8.357 & kfar & srby87 & 9.5 & 5.9 & 5.61 & 8.9 & 5.5 & 4.92 & 9.5 & 5.9 & 5.61 \\
\hline 38.25 & -0.15 & 65.00 & 32.70 & 11.10 & 1.995 & kfar & srby 87 & 9.0 & 5.8 & 1.31 & 8.5 & 5.4 & 1.17 & 9.1 & 5.8 & 1.34 \\
\hline 38.90 & 0.40 & 32.00 & & 2.20 & 5.754 & knear & dect86 & 2.2 & 6.9 & 5.75 & 2.1 & 6.5 & 5.24 & 2.2 & 6.9 & 5.75 \\
\hline 38.95 & -0.45 & 42.00 & 16.30 & 2.90 & 0.994 & knear & srby 87 & 2.8 & 6.5 & 0.93 & 2.7 & 6.1 & 0.86 & 2.8 & 6.6 & 0.93 \\
\hline 39.85 & -0.20 & 57.00 & 1718.00 & 11.30 & 104.798 & kfar & srby 87 & 9.2 & 6.1 & 69.47 & 8.6 & 5.7 & 60.70 & 9.2 & 6.1 & 69.47 \\
\hline 40.30 & -0.45 & 74.00 & 14.70 & 5.70 & 0.897 & knear & srby 87 & 5.5 & 5.6 & 0.84 & 5.1 & 5.3 & 0.72 & 5.0 & 5.7 & 0.69 \\
\hline 41.00 & -0.20 & 38.00 & 16.10 & 2.70 & 0.982 & knear & srby 87 & 2.6 & 6.8 & 0.91 & 2.5 & 6.3 & 0.84 & 2.6 & 6.8 & 0.91 \\
\hline 41.15 & -0.20 & 60.00 & 464.00 & 10.70 & 28.304 & kfar & srby87 & 8.6 & 6.0 & 18.28 & 8.1 & 5.7 & 16.22 & 8.6 & 6.0 & 18.28 \\
\hline 41.90 & -0.40 & 59.00 & 84.30 & 10.60 & 5.142 & kfar & srby 87 & 8.5 & 6.1 & 3.31 & 8.0 & 5.7 & 2.93 & 8.5 & 6.1 & 3.31 \\
\hline 42.15 & -0.60 & 67.00 & 50.30 & 5.40 & 3.068 & knear & srby 87 & 5.0 & 5.8 & 2.63 & 4.7 & 5.5 & 2.32 & 4.7 & 5.9 & 2.32 \\
\hline 42.35 & -0.05 & 58.00 & 80.20 & 10.50 & 4.892 & kfar & srby87 & 8.4 & 6.1 & 3.13 & 7.9 & 5.8 & 2.77 & 8.5 & 6.1 & 3.21 \\
\hline 42.75 & -0.35 & 59.00 & 39.80 & 4.60 & 2.428 & knear & srby87 & 4.3 & 6.1 & 2.12 & 4.0 & 5.7 & 1.84 & 4.1 & 6.2 & 1.93 \\
\hline 43.10 & 0.05 & 12.00 & 102.00 & 13.80 & 6.222 & kfar & srby87 & 11.7 & 8.0 & 4.47 & 10.8 & 7.4 & 3.81 & 11.2 & 7.7 & 4.10 \\
\hline 43.20 & -0.50 & 57.00 & 7.50 & 4.40 & 0.457 & knear & srby87 & 4.1 & 6.2 & 0.40 & 3.9 & 5.8 & 0.36 & 4.0 & 6.2 & 0.38 \\
\hline 44.35 & -0.20 & 64.00 & 426.00 & 9.20 & 25.986 & kfar & srby87 & 7.0 & 6.0 & 15.04 & 6.5 & 5.7 & 12.97 & 7.2 & 6.0 & 15.92 \\
\hline 45.45 & 0.05 & 58.00 & 289.00 & 9.50 & 17.629 & kfar & srby 87 & 7.4 & 6.2 & 10.70 & 6.9 & 5.8 & 9.30 & 7.4 & 6.2 & 10.70 \\
\hline 46.00 & 0.20 & 25.00 & & 1.80 & 0.513 & knear & dect86 & 1.7 & 7.4 & 0.46 & 1.7 & 6.9 & 0.46 & 1.9 & 7.3 & 0.57 \\
\hline 46.30 & -0.20 & 59.00 & 46.50 & 9.10 & 2.836 & kfar & srby 87 & 6.8 & 6.2 & 1.58 & 6.3 & 5.8 & 1.36 & 6.9 & 6.2 & 1.63 \\
\hline 47.05 & 0.25 & 55.00 & 1.80 & 9.30 & 2.550 & kfar & 87 & 7.1 & 6.4 & 1.49 & 6.6 & 6.0 & 1.28 & 7.1 & 6.4 & 1.49 \\
\hline 48.60 & 0.25 & 10.00 & 36.50 & 12.50 & 2.226 & kfar & srby 87 & 10.6 & 8.1 & 1.60 & 9.9 & 7.5 & 1.40 & 10.2 & 7.8 & 1.48 \\
\hline 48.60 & 0.00 & 20.00 & 63.40 & 11.80 & 3.867 & kfar & srby 87 & 9.9 & 7.7 & 2.72 & 9.2 & 7.1 & 2.35 & 9.5 & 7.5 & 2.51 \\
\hline 48.85 & 0.15 & 51.00 & 38.70 & 6.60 & 2.361 & ktan & srby 87 & & & & & & & & & \\
\hline 49.50 & -0.40 & 57.00 & 665.00 & 6.60 & 40.565 & ktan & srby87 & 5.5 & 6.5 & 28.17 & 5.2 & 6.1 & 25.18 & 5.5 & 6.4 & 28.17 \\
\hline 49.75 & -0.55 & 68.00 & 15.40 & 6.50 & 0.939 & ktan & srby87 & 5.5 & 6.5 & 0.67 & 5.2 & 6.1 & 0.60 & 5.4 & 6.4 & 0.65 \\
\hline 50.05 & 0.60 & -2.00 & 112.00 & 13.00 & 6.832 & kout & srby 87 & 11.3 & 8.7 & 5.16 & 10.4 & 8.1 & 4.37 & 10.7 & 8.4 & 4.63 \\
\hline 50.20 & -0.50 & 45.00 & & 3.60 & 2.512 & kfar & $\operatorname{dect} 86$ & 7.2 & 6.8 & 10.05 & 6.6 & 6.3 & 8.44 & 6.9 & 6.7 & 9.23 \\
\hline 50.85 & 0.25 & 43.00 & 5.70 & 3.60 & 0.348 & knear & srby87 & 3.6 & 6.8 & 0.35 & 3.5 & 6.4 & 0.33 & 3.7 & 6.8 & 0.37 \\
\hline 51.35 & -0.05 & 54.00 & 71.30 & 6.30 & 4.349 & ktan & srby87 & 5.3 & 6.6 & 3.08 & 5.0 & 6.2 & 2.74 & 5.3 & 6.6 & 3.08 \\
\hline 52.10 & -0.30 & 59.00 & & 6.10 & 18.197 & ktan & $\operatorname{dect} 86$ & 5.2 & 6.7 & 13.22 & 4.9 & 6.3 & 11.74 & 5.2 & 6.6 & 13.22 \\
\hline 52.30 & -0.05 & 52.00 & 7.30 & 6.10 & 0.445 & ktan & srby 87 & 5.2 & 6.7 & 0.32 & 4.9 & 6.3 & 0.29 & 5.1 & 6.7 & 0.31 \\
\hline 52.55 & -0.95 & 64.00 & 97.20 & 6.10 & 5.929 & ktan & srby87 & 5.2 & 6.7 & 4.31 & 4.9 & 6.4 & 3.83 & 5.1 & 6.7 & 4.14 \\
\hline 53.15 & -0.25 & 61.00 & 11.70 & 6.00 & 0.714 & ktan & srby87 & 5.1 & 6.8 & 0.52 & 4.8 & 6.4 & 0.46 & 5.0 & 6.7 & 0.50 \\
\hline 53.55 & 0.05 & 23.00 & 11.10 & 1.80 & 0.677 & knear & srby87 & 1.7 & 7.6 & 0.60 & 1.8 & 7.1 & 0.68 & 2.0 & 7.5 & 0.84 \\
\hline 54.10 & -0.05 & 40.00 & 21.90 & 8.20 & 1.336 & kfar & srby 87 & 6.2 & 7.0 & 0.76 & 5.5 & 6.5 & 0.60 & 5.7 & 6.9 & 0.65 \\
\hline 54.65 & 0.80 & 31.00 & 67.10 & 9.00 & 4.093 & kfar & srby87 & 7.2 & 7.3 & 2.62 & 6.6 & 6.8 & 2.20 & 6.8 & 7.2 & 2.34 \\
\hline 55.30 & 0.20 & 30.00 & 72.60 & 9.00 & 4.429 & kfar & srby 87 & 7.1 & 7.4 & 2.76 & 6.5 & 6.9 & 2.31 & 6.7 & 7.2 & 2.45 \\
\hline 55.70 & 0.00 & 36.00 & & 5.60 & 8.318 & ktan & $\operatorname{dect} 86$ & & & & & & & & & \\
\hline 56.80 & 1.90 & -54.60 & & 14.40 & 1.400 & kout & mk88 & 14.9 & 12.5 & 1.50 & 13.6 & 11.4 & 1.25 & 13.4 & 11.3 & 1.21 \\
\hline 59.30 & -0.20 & 28.00 & 6.10 & 2.70 & 0.372 & knear & srby 87 & 2.8 & 7.5 & 0.40 & 3.0 & 7.0 & 0.46 & 3.5 & 7.4 & 0.63 \\
\hline 59.90 & 1.50 & -65.00 & & 14.90 & 0.580 & kout & mk88 & 15.4 & 13.3 & 0.62 & 14.0 & 12.1 & 0.51 & 13.7 & 11.9 & 0.49 \\
\hline 60.00 & 0.10 & 22.00 & 110.00 & 8.00 & 6.710 & kfar & srby 87 & 6.5 & 7.7 & 4.43 & 5.9 & 7.2 & 3.65 & 5.8 & 7.5 & 3.53 \\
\hline
\end{tabular}


Table A.2. continued.

\begin{tabular}{|c|c|c|c|c|c|c|c|c|c|c|c|c|c|c|c|c|}
\hline $\begin{array}{r}l \\
\left({ }^{\circ}\right) \\
(1)\end{array}$ & $\begin{array}{r}b \\
\left({ }^{\circ}\right) \\
(2)\end{array}$ & $\begin{array}{r}V_{\mathrm{lsr}} \\
\mathrm{km} \mathrm{s}^{-1} \\
\text { (3) }\end{array}$ & $\begin{array}{r}L_{\mathrm{CO}} \\
10^{3} \mathrm{~K} \mathrm{~km} \mathrm{~s}^{-1} \mathrm{pc}^{2} \\
(4)\end{array}$ & $\begin{array}{r}D \\
\mathrm{kpc} \\
(5)\end{array}$ & $\begin{array}{r}M_{\mathrm{GMCs}} \\
10^{5} M_{\odot} \\
(6)\end{array}$ & Mark & Ref. & $\begin{array}{r}D_{8.5} \\
\mathrm{kpc} \\
(9)\end{array}$ & $\begin{array}{l}R_{8.5} \\
\mathrm{kpc} \\
(10)\end{array}$ & $\begin{array}{r}M_{8.5} \\
10^{5} M_{\odot} \\
(11)\end{array}$ & $\begin{array}{l}D_{8.0} \\
\mathrm{kpc} \\
(12)\end{array}$ & $\begin{array}{l}R_{8.0} \\
\mathrm{kpc} \\
(13)\end{array}$ & $\begin{array}{r}M_{8.0} \\
10^{5} M_{\odot} \\
(14)\end{array}$ & $\begin{array}{l}D_{8.4} \\
\mathrm{kpc} \\
(15)\end{array}$ & $\begin{array}{l}R_{8.4} \\
\mathrm{kpc} \\
(16)\end{array}$ & $\begin{array}{r}M_{8.4} \\
10^{5} M_{\odot} \\
(17)\end{array}$ \\
\hline 60.90 & -0.10 & 23.00 & 13.80 & 7.50 & 0.842 & kfar & srby87 & 6.1 & 7.7 & 0.56 & 5.4 & 7.1 & 0.44 & 5.2 & 7.5 & 0.40 \\
\hline 61.50 & 0.10 & 22.00 & 32.90 & 7.40 & 2.007 & kfar & srby 87 & 6.0 & 7.7 & 1.32 & 5.3 & 7.2 & 1.03 & 5.1 & 7.5 & 0.95 \\
\hline 63.10 & 0.40 & 20.00 & 21.20 & 7.00 & 1.293 & kfar & srby 87 & 5.7 & 7.8 & 0.86 & 5.0 & 7.3 & 0.66 & 4.7 & 7.6 & 0.58 \\
\hline 63.50 & 0.00 & -9.70 & & 8.49 & 38.019 & kout & sod91 & 8.8 & 9.1 & 40.85 & 8.0 & 8.4 & 33.76 & 8.0 & 8.7 & 33.76 \\
\hline 65.50 & 1.40 & -72.20 & 29.00 & 12.00 & 5.800 & kout & dbt90 & 14.9 & 13.7 & 8.94 & 13.5 & 12.5 & 7.34 & 13.0 & 12.2 & 6.81 \\
\hline 65.95 & 0.85 & -63.00 & 4.40 & 12.00 & 0.900 & kout & $\mathrm{dbt} 90$ & 13.6 & 12.8 & 1.16 & 12.3 & 11.6 & 0.95 & 12.0 & 11.5 & 0.90 \\
\hline 66.45 & 1.75 & -60.90 & 7.30 & 12.00 & 1.400 & kout & dbt90 & 13.2 & 12.5 & 1.69 & 12.0 & 11.5 & 1.40 & 11.7 & 11.4 & 1.33 \\
\hline 66.70 & 1.45 & -69.10 & 4.50 & 12.00 & 0.900 & kout & dbt90 & 14.2 & 13.3 & 1.26 & 12.8 & 12.2 & 1.02 & 12.4 & 12.0 & 0.96 \\
\hline 67.00 & 1.15 & -68.00 & 3.30 & 12.00 & 0.700 & kout & dbt90 & 14.0 & 13.2 & 0.95 & 12.6 & 12.0 & 0.77 & 12.2 & 11.9 & 0.72 \\
\hline 67.50 & 1.00 & -51.10 & 8.00 & 12.00 & 1.600 & kout & dbt90 & 11.9 & 11.7 & 1.57 & 10.8 & 10.7 & 1.30 & 10.5 & 10.7 & 1.23 \\
\hline 68.16 & 0.90 & -63.90 & 4.90 & 12.00 & 1.000 & kout & dbt90 & 13.2 & 12.8 & 1.21 & 11.9 & 11.6 & 0.98 & 11.6 & 11.5 & 0.93 \\
\hline 68.19 & 1.24 & -60.90 & 14.00 & 12.00 & 2.900 & kout & dbt90 & 12.8 & 12.5 & 3.30 & 11.6 & 11.4 & 2.71 & 11.3 & 11.3 & 2.57 \\
\hline 68.20 & 0.90 & -63.40 & 2.50 & 12.00 & 0.500 & kout & $\mathrm{dbt} 90$ & 13.1 & 12.7 & 0.60 & 11.9 & 11.6 & 0.49 & 11.5 & 11.5 & 0.46 \\
\hline 68.30 & 1.25 & -62.60 & 26.00 & 12.00 & 5.200 & kout & dbt90 & 13.0 & 12.6 & 6.10 & 11.8 & 11.5 & 5.03 & 11.4 & 11.4 & 4.69 \\
\hline 68.37 & 1.26 & -64.00 & 9.30 & 12.00 & 1.800 & kout & $\mathrm{dbt} 90$ & 13.1 & 12.8 & 2.15 & 11.9 & 11.6 & 1.77 & 11.5 & 11.5 & 1.65 \\
\hline 68.56 & 1.22 & -61.90 & 0.90 & 12.00 & 0.200 & kout & $\mathrm{dbt} 90$ & 12.9 & 12.6 & 0.23 & 11.6 & 11.5 & 0.19 & 11.3 & 11.4 & 0.18 \\
\hline 69.00 & 0.80 & -62.30 & 6.10 & 12.00 & 1.200 & kout & dbt 90 & 12.8 & 12.6 & 1.37 & 11.6 & 11.5 & 1.12 & 11.2 & 11.4 & 1.05 \\
\hline 69.30 & 0.85 & -63.00 & 4.40 & 12.00 & 0.900 & kout & dbt90 & 12.8 & 12.6 & 1.02 & 11.6 & 11.5 & 0.84 & 11.2 & 11.4 & 0.78 \\
\hline 69.44 & 1.39 & -67.70 & 2.20 & 12.00 & 0.400 & kout & $\mathrm{dbt} 90$ & 13.4 & 13.1 & 0.50 & 12.1 & 11.9 & 0.41 & 11.6 & 11.8 & 0.37 \\
\hline 69.74 & 1.46 & -64.20 & 12.00 & 12.00 & 2.300 & kout & $\mathrm{dbt} 90$ & 12.9 & 12.7 & 2.66 & 11.6 & 11.6 & 2.15 & 11.2 & 11.5 & 2.00 \\
\hline 69.83 & 1.27 & -60.60 & 4.20 & 12.00 & 0.800 & kout & $\mathrm{dbt} 90$ & 12.4 & 12.4 & 0.85 & 11.2 & 11.3 & 0.70 & 10.9 & 11.2 & 0.66 \\
\hline 69.85 & 1.50 & -63.60 & 22.00 & 12.00 & 4.400 & kout & dbt 90 & 12.8 & 12.7 & 5.01 & 11.5 & 11.6 & 4.04 & 11.1 & 11.5 & 3.76 \\
\hline 69.85 & 1.01 & -57.30 & 1.00 & 12.00 & 0.200 & kout & dbt90 & 12.0 & 12.1 & 0.20 & 10.9 & 11.1 & 0.17 & 10.6 & 11.0 & 0.16 \\
\hline 69.90 & 0.95 & -65.30 & 19.00 & 12.00 & 3.800 & kout & dbt90 & 12.9 & 12.8 & 4.39 & 11.7 & 11.7 & 3.61 & 11.3 & 11.6 & 3.37 \\
\hline 69.92 & 1.50 & -63.80 & 4.80 & 12.00 & 1.000 & kout & dbt90 & 12.8 & 12.7 & 1.14 & 11.5 & 11.6 & 0.92 & 11.1 & 11.5 & 0.86 \\
\hline 70.01 & 1.04 & -63.60 & 6.30 & 12.00 & 1.200 & kout & $\mathrm{dbt} 90$ & 12.7 & 12.7 & 1.34 & 11.5 & 11.6 & 1.10 & 11.1 & 11.4 & 1.03 \\
\hline 70.02 & 1.17 & -67.10 & 11.00 & 12.00 & 2.200 & kout & dbt 90 & 13.1 & 13.0 & 2.62 & 11.9 & 11.8 & 2.16 & 11.4 & 11.7 & 1.99 \\
\hline 70.05 & 1.15 & -66.50 & 19.00 & 12.00 & 3.800 & kout & dbt 90 & 13.1 & 12.9 & 4.53 & 11.8 & 11.8 & 3.67 & 11.4 & 11.7 & 3.43 \\
\hline 70.16 & 1.17 & -66.90 & 6.10 & 12.00 & 1.200 & kout & $\mathrm{dbt} 90$ & 13.1 & 13.0 & 1.43 & 11.8 & 11.8 & 1.16 & 11.4 & 11.7 & 1.08 \\
\hline 70.16 & 1.29 & -64.80 & 2.60 & 12.00 & 0.500 & kout & $\mathrm{dbt} 90$ & 12.8 & 12.8 & 0.57 & 11.6 & 11.6 & 0.47 & 11.2 & 11.5 & 0.44 \\
\hline 70.30 & 1.12 & -65.70 & 7.10 & 12.00 & 1.400 & kout & $\mathrm{dbt} 90$ & 12.9 & 12.8 & 1.62 & 11.7 & 11.7 & 1.33 & 11.2 & 11.6 & 1.22 \\
\hline 70.33 & 0.96 & -62.90 & 5.60 & 12.00 & 1.100 & kout & $\mathrm{dbt} 90$ & 12.6 & 12.6 & 1.21 & 11.4 & 11.5 & 0.99 & 11.0 & 11.4 & 0.92 \\
\hline 70.40 & 0.50 & -70.80 & 6.80 & 12.00 & 1.400 & kout & dbt 90 & 13.5 & 13.3 & 1.77 & 12.2 & 12.1 & 1.45 & 11.7 & 11.9 & 1.33 \\
\hline 70.40 & 0.90 & -62.60 & 14.00 & 12.00 & 2.700 & kout & dbt 90 & 12.5 & 12.5 & 2.93 & 11.3 & 11.5 & 2.39 & 10.9 & 11.4 & 2.23 \\
\hline 70.53 & 1.15 & -59.20 & 4.80 & 12.00 & 1.000 & kout & dbt90 & 12.1 & 12.2 & 1.02 & 10.9 & 11.2 & 0.83 & 10.6 & 11.1 & 0.78 \\
\hline 70.90 & 0.55 & -71.00 & 15.00 & 12.00 & 2.900 & kout & $\mathrm{dbt} 90$ & 13.4 & 13.3 & 3.62 & 12.1 & 12.1 & 2.95 & 11.6 & 11.9 & 2.71 \\
\hline 71.10 & -0.40 & 10.00 & 5.20 & 1.40 & 0.317 & knear & srby 87 & 1.1 & 8.2 & 0.20 & 1.6 & 7.6 & 0.41 & 2.7 & 8.0 & 1.18 \\
\hline 74.10 & 1.50 & -36.20 & & 8.40 & 0.400 & kout & $\mathrm{mk} 88$ & 8.9 & 10.5 & 0.45 & 8.0 & 9.6 & 0.36 & 7.8 & 9.8 & 0.34 \\
\hline 75.00 & 1.90 & -79.20 & & 13.10 & 0.150 & kout & mk88 & 13.6 & 14.0 & 0.16 & 12.2 & 12.7 & 0.13 & 11.6 & 12.5 & 0.12 \\
\hline 76.20 & 0.10 & -2.00 & 72.00 & 5.10 & 4.392 & kout & srby 87 & 4.8 & 8.7 & 3.89 & 4.1 & 8.1 & 2.84 & 3.5 & 8.4 & 2.07 \\
\hline 76.30 & -0.70 & -1.00 & 43.20 & 4.90 & 2.635 & kout & srby 87 & 4.7 & 8.7 & 2.42 & 3.9 & 8.0 & 1.67 & 3.3 & 8.4 & 1.20 \\
\hline 77.20 & 0.70 & 2.00 & 75.70 & 4.10 & 4.618 & kout & srby 87 & 4.0 & 8.5 & 4.40 & & & & 1.9 & 8.3 & 0.99 \\
\hline 78.00 & -0.30 & -4.00 & 19.80 & 4.80 & 1.208 & kout & srby 87 & 4.7 & 8.8 & 1.16 & 3.9 & 8.2 & 0.80 & 3.3 & 8.5 & 0.57 \\
\hline 78.10 & -0.80 & 0.00 & 29.70 & 4.10 & 1.812 & kout & srby 87 & 4.1 & 8.6 & 1.81 & & & & 2.1 & 8.3 & 0.48 \\
\hline 78.20 & 0.10 & 11.00 & 11.30 & 2.00 & 0.689 & ktan & srby 87 & 1.7 & 8.3 & 0.50 & 1.6 & 7.8 & 0.44 & 1.7 & 8.2 & 0.50 \\
\hline 78.80 & 1.70 & -76.00 & & 11.80 & 0.700 & kout & mk88 & 12.3 & 13.6 & 0.76 & 11.1 & 12.3 & 0.62 & 10.5 & 12.1 & 0.55 \\
\hline 78.80 & -0.50 & -1.00 & 42.10 & 4.10 & 2.568 & kout & srby 87 & 4.0 & 8.7 & 2.44 & 3.3 & 8.0 & 1.66 & 2.3 & 8.4 & 0.81 \\
\hline 79.20 & 0.10 & 8.00 & 6.40 & 1.90 & 0.390 & ktan & srby 87 & 1.6 & 8.3 & 0.28 & 1.5 & 7.9 & 0.24 & 1.6 & 8.3 & 0.28 \\
\hline 79.60 & -0.60 & 4.00 & 12.10 & 2.50 & 0.738 & kfar & srby 87 & 2.9 & 8.5 & 0.99 & 1.9 & 7.9 & 0.43 & 1.5 & 8.4 & 0.27 \\
\hline 80.10 & 1.90 & -72.10 & & 11.20 & 0.200 & kout & mk88 & 11.6 & 13.2 & 0.21 & 10.4 & 12.0 & 0.17 & 9.8 & 11.8 & 0.15 \\
\hline 80.70 & 0.70 & -1.00 & 14.10 & 3.50 & 0.860 & kout & srby 87 & 3.6 & 8.7 & 0.91 & 2.8 & 8.0 & 0.55 & 1.4 & 8.4 & 0.14 \\
\hline 80.80 & -0.50 & -3.00 & 3.60 & 3.80 & 0.220 & kout & srby 87 & 3.9 & 8.8 & 0.23 & 3.1 & 8.1 & 0.15 & 2.2 & 8.4 & 0.07 \\
\hline 81.20 & 1.00 & 13.00 & 9.00 & 1.50 & 0.549 & ktan & srby 87 & 1.3 & 8.4 & 0.41 & 1.2 & 7.9 & 0.35 & 1.3 & 8.3 & 0.41 \\
\hline 81.40 & 0.70 & -2.00 & 10.30 & 3.50 & 0.628 & kout & srby 87 & 3.6 & 8.7 & 0.66 & 2.8 & 8.1 & 0.40 & 1.3 & 8.4 & 0.09 \\
\hline 81.40 & 0.00 & -4.00 & 3.60 & 2.00 & 0.220 & kout & srby 87 & 3.9 & 8.8 & 0.84 & 3.2 & 8.1 & 0.56 & 2.3 & 8.5 & 0.29 \\
\hline 81.70 & 0.60 & -2.00 & 42.90 & 2.00 & 2.617 & kout & srby 87 & 3.5 & 8.7 & 8.01 & 2.7 & 8.1 & 4.77 & 1.2 & 8.4 & 0.94 \\
\hline 82.00 & -0.40 & 5.00 & 4.10 & 1.40 & 0.250 & ktan & srby 87 & & & & 1.1 & 7.9 & 0.15 & 1.2 & 8.3 & 0.18 \\
\hline 83.30 & 1.90 & -72.30 & & 10.60 & 0.200 & kout & $\mathrm{mk} 88$ & 11.0 & 13.1 & 0.22 & 9.9 & 12.0 & 0.17 & 9.2 & 11.8 & 0.15 \\
\hline 83.50 & -1.00 & 2.00 & 2.00 & 1.10 & 0.122 & kout & srby 87 & 2.3 & 8.5 & 0.53 & & & & 0.9 & 8.4 & 0.08 \\
\hline 84.60 & 0.20 & -1.00 & 48.60 & 2.00 & 2.965 & kout & srby 87 & 2.7 & 8.7 & 5.40 & 1.8 & 8.0 & 2.40 & 0.8 & 8.5 & 0.47 \\
\hline 84.70 & 1.70 & -83.00 & & 11.40 & 1.800 & kout & $\mathrm{mk} 88$ & 12.2 & 14.2 & 2.06 & 10.9 & 12.9 & 1.65 & 10.1 & 12.6 & 1.41 \\
\hline 85.00 & 2.40 & -51.00 & & 7.80 & 0.200 & kout & $\mathrm{mk} 88$ & 8.3 & 11.4 & 0.23 & 7.4 & 10.4 & 0.18 & 7.0 & 10.5 & 0.16 \\
\hline 85.00 & 2.40 & -82.80 & & 11.50 & 0.300 & kout & mk88 & 12.1 & 14.2 & 0.33 & 10.8 & 12.9 & 0.26 & 10.0 & 12.6 & 0.23 \\
\hline 85.40 & 0.00 & -38.00 & 6.10 & 7.10 & 0.372 & kout & srby 87 & 6.9 & 10.5 & 0.35 & 6.1 & 9.7 & 0.27 & 5.7 & 9.8 & 0.24 \\
\hline 86.00 & 0.50 & -16.80 & & 4.18 & 5.495 & kout & sod91 & 4.6 & 9.4 & 6.65 & 3.9 & 8.7 & 4.78 & 3.4 & 8.9 & 3.64 \\
\hline
\end{tabular}


Table A.2. continued.

\begin{tabular}{|c|c|c|c|c|c|c|c|c|c|c|c|c|c|c|c|c|}
\hline $\begin{array}{r}l \\
\left({ }^{\circ}\right) \\
(1) \\
\end{array}$ & $\begin{array}{r}b \\
\left({ }^{\circ}\right) \\
(2) \\
\end{array}$ & $\begin{array}{r}V_{\text {lsr }} \\
\mathrm{km} \mathrm{s}^{-1} \\
(3) \\
\end{array}$ & $\begin{array}{r}L_{\mathrm{CO}} \\
10^{3} \mathrm{~K} \mathrm{~km} \mathrm{~s}^{-1} \mathrm{pc}^{2} \\
(4)\end{array}$ & $\begin{array}{r}D \\
\mathrm{kpc} \\
(5) \\
\end{array}$ & $\begin{array}{r}M_{\mathrm{GMCs}} \\
10^{5} M_{\odot} \\
(6) \\
\end{array}$ & Mark & Ref. & $\begin{array}{r}D_{8.5} \\
\mathrm{kpc} \\
(9) \\
\end{array}$ & $\begin{array}{l}R_{8.5} \\
\mathrm{kpc} \\
(10)\end{array}$ & $\begin{array}{r}M_{8.5} \\
10^{5} M_{\odot} \\
(11) \\
\end{array}$ & $\begin{array}{l}D_{8.0} \\
\mathrm{kpc} \\
(12) \\
\end{array}$ & $\begin{array}{l}R_{8.0} \\
\mathrm{kpc} \\
(13) \\
\end{array}$ & $\begin{array}{r}M_{8.0} \\
10^{5} M_{\odot} \\
(14)\end{array}$ & $\begin{array}{l}D_{8.4} \\
\mathrm{kpc} \\
(15) \\
\end{array}$ & $\begin{array}{l}R_{8.4} \\
\mathrm{kpc} \\
(16) \\
\end{array}$ & $\begin{array}{r}M_{8.4} \\
10^{5} M_{\odot} \\
(17)\end{array}$ \\
\hline 88.40 & 1.90 & -63.70 & & 8.60 & 0.200 & kout & $\mathrm{mk} 88$ & 9.2 & 12.3 & 0.23 & 8.2 & 11.3 & 0.18 & 7.6 & 11.2 & 0.16 \\
\hline 88.80 & 1.90 & -60.60 & & 8.30 & 0.120 & kout & mk88 & 8.8 & 12.1 & 0.13 & 7.8 & 11.0 & 0.11 & 7.2 & 11.0 & 0.09 \\
\hline 89.90 & 1.30 & -71.00 & & 9.20 & 0.630 & kout & mk88 & 9.8 & 12.9 & 0.71 & 8.7 & 11.8 & 0.56 & 8.0 & 11.7 & 0.48 \\
\hline 91.00 & -0.50 & -24.60 & & 4.25 & 7.413 & kout & sod91 & 4.7 & 9.8 & 9.07 & 4.0 & 9.0 & 6.57 & 3.5 & 9.2 & 5.03 \\
\hline 91.00 & -1.00 & -36.30 & & 5.46 & 8.710 & kout & sod91 & 5.9 & 10.4 & 10.17 & 5.1 & 9.6 & 7.60 & 4.6 & 9.7 & 6.18 \\
\hline 92.90 & 1.90 & -87.70 & & 10.40 & 0.200 & kout & $\mathrm{mk} 88$ & 11.5 & 14.7 & 0.24 & 10.2 & 13.3 & 0.19 & 9.4 & 13.0 & 0.16 \\
\hline 93.20 & 1.70 & -71.20 & & 9.00 & 0.200 & kout & $\mathrm{mk} 88$ & 9.3 & 13.0 & 0.21 & 8.3 & 11.8 & 0.17 & 7.6 & 11.7 & 0.14 \\
\hline 103.00 & 2.00 & -18.30 & & 2.31 & 3.802 & kout & sod91 & 2.7 & 9.5 & 5.19 & 2.2 & 8.7 & 3.45 & 1.7 & 9.0 & 2.06 \\
\hline 106.16 & 0.99 & -60.70 & 0.98 & 6.33 & 0.119 & kout & hcs01 & 6.8 & 12.3 & 0.14 & 5.9 & 11.2 & 0.10 & 5.3 & 11.2 & 0.08 \\
\hline 106.21 & 1.47 & -22.38 & 0.66 & 2.50 & 0.080 & kout & hes 01 & 2.9 & 9.7 & 0.11 & 2.4 & 8.9 & 0.07 & 1.9 & 9.2 & 0.05 \\
\hline 106.27 & -0.01 & -56.36 & 3.20 & 5.86 & 0.387 & kout & hes01 & 6.3 & 11.9 & 0.45 & 5.5 & 10.9 & 0.34 & 4.9 & 10.9 & 0.27 \\
\hline 106.36 & 1.34 & -25.49 & 0.79 & 2.80 & 0.096 & kout & hcs 01 & 3.2 & 9.9 & 0.13 & 2.6 & 9.1 & 0.08 & 2.2 & 9.3 & 0.06 \\
\hline 106.44 & 0.41 & -55.63 & 1.20 & 5.77 & 145 & kout & hcs 01 & 6.2 & 11.9 & 0.17 & 5.4 & 10.9 & 0.13 & 4.8 & 10.9 & 0.10 \\
\hline 106.48 & 0.90 & -54.17 & 2.70 & 5.61 & 327 & kout & hes 01 & 6.1 & 11.8 & 0.39 & 5.3 & 10.8 & 0.29 & 4.7 & 10.8 & 0.23 \\
\hline 106.51 & 1.09 & -60.92 & 1.70 & 6.33 & 0.206 & kout & hcs01 & 6.8 & 12.3 & 0.24 & 5.9 & 11.2 & 0.18 & 5.3 & 11.2 & 0.14 \\
\hline 106.59 & 1.01 & -61.62 & 2.90 & 6.40 & 0.351 & kout & hcs01 & 6.9 & 12.4 & 0.41 & 6.0 & 11.3 & 0.31 & 5.4 & 11.3 & 0.25 \\
\hline 106.61 & 1.02 & -11.89 & 1.10 & 1.42 & 0.133 & kout & hcs01 & 1.8 & 9.2 & 0.21 & 1.3 & 8.5 & 0.11 & 0.8 & 8.8 & 0.04 \\
\hline 106.89 & -1.03 & -37.89 & 0.76 & 3.96 & 0.092 & kout & hcs01 & 4.3 & 10.6 & 0.11 & 3.7 & 9.8 & 0.08 & 3.2 & 9.9 & 0.06 \\
\hline 106.98 & 5.26 & -8.53 & 1.60 & 1.05 & 0.194 & kout & hcs01 & 1.4 & 9.0 & 0.34 & 1.0 & 8.3 & 0.18 & 0.5 & 8.7 & 0.04 \\
\hline 107.16 & 0.67 & -52.78 & 1.40 & 5.42 & 0.169 & kout & hcs 01 & 5.9 & 11.7 & 0.20 & 5.1 & 10.7 & 0.15 & 4.5 & 10.7 & 0.12 \\
\hline 107.17 & 0.01 & -50.58 & 2.50 & 5.19 & 0.303 & kout & hes01 & 5.6 & 11.5 & 0.35 & 4.9 & 10.5 & 0.27 & 4.3 & 10.6 & 0.21 \\
\hline 107.17 & 4.52 & -6.33 & 1.00 & 0.79 & 0.121 & kout & & 1.2 & 8.9 & 0.28 & 0.7 & 8.2 & 0.10 & 0.3 & 8.6 & 0.02 \\
\hline 107.23 & -0.93 & -41.58 & 1.60 & 4.29 & 0.194 & kout & & 4.7 & 10.9 & 0.23 & 4.0 & 10.0 & 0.17 & 3.5 & 10.1 & 0.13 \\
\hline 107.36 & 0.15 & -50.66 & 0.81 & 5.19 & 0.098 & kout & & 5.6 & 11.5 & 0.11 & 4.9 & 10.5 & 0.09 & 4.3 & 10.6 & 0.07 \\
\hline 107.40 & -0.14 & 50.69 & 2.10 & 5.19 & 0.254 & kout & & 5.6 & 11.5 & 0.30 & 4.9 & 10.5 & 0.23 & 4.3 & 10.6 & 0.17 \\
\hline 7.44 & 0.50 & 50.76 & 0.72 & 5.19 & 087 & & & 5.6 & 11.5 & 0.10 & 4.9 & 10.5 & 08 & 4.3 & 10.6 & 0.06 \\
\hline 107.66 & -2.27 & -55.19 & 1.80 & 5.65 & 218 & kout & & 6.1 & 11.9 & 0.25 & 5.3 & 10.9 & 0.19 & 4.7 & 10.9 & 0.15 \\
\hline 107.81 & 0.74 & 52.20 & 0.75 & 5.32 & 0.091 & kout & & 5.8 & 11.6 & 0.11 & 5.0 & 10.7 & 0.08 & 4.4 & 10.7 & 0.06 \\
\hline 108.03 & 1.04 & -57.45 & 0.73 & 5.86 & 0.088 & kout & hes01 & 6.3 & 12.1 & 0.10 & 5.5 & 11.0 & 0.08 & 4.9 & 11.0 & 0.06 \\
\hline 108.36 & 0.43 & -47.77 & 0.76 & 4.83 & 0.092 & kout & hcs01 & 5.3 & 11.3 & 0.11 & 4.5 & 10.4 & 0.08 & 4.0 & 10.5 & 0.06 \\
\hline 108.47 & -2.85 & -53.70 & 0.84 & 5.44 & 0.102 & kout & hcs 01 & 5.9 & 11.8 & 0.12 & 5.1 & 10.8 & 0.09 & 4.5 & 10.8 & 0.07 \\
\hline 108.54 & -1.02 & -51.98 & 31.00 & 5.25 & 3.751 & kout & hcs01 & 5.7 & 11.6 & 4.42 & 4.9 & 10.7 & 3.27 & 4.3 & 10.7 & 2.52 \\
\hline 108.63 & -0.17 & -50.32 & 0.71 & 5.07 & 0.086 & kout & hcs 01 & 5.5 & 11.5 & 0.10 & 4.8 & 10.5 & 0.08 & 4.2 & 10.6 & 0.06 \\
\hline 108.64 & 0.73 & -54.88 & 3.40 & 5.54 & 411 & kout & hes 01 & 6.0 & 11.9 & 0.48 & 5.2 & 10.9 & 0.36 & 4.6 & 10.9 & 0.28 \\
\hline 8.71 & -1.13 & -50.13 & 2.30 & 5.05 & 278 & kout & & 5.5 & 11.5 & 0.33 & 4.7 & 10.5 & 0.24 & 4.2 & 10.6 & 0.19 \\
\hline 3.73 & -2.63 & -55.66 & 40 & 5.63 & 411 & kout & & 6.1 & 11.9 & 0.48 & 5.3 & 10.9 & 0.36 & 4.6 & 10.9 & 0.27 \\
\hline 3.76 & 2.70 & -11.11 & 1.60 & 1.25 & 194 & kout & & 1.6 & 9.1 & 0.32 & 1.2 & 8.5 & 18 & 0.7 & 8.8 & 0.06 \\
\hline 8.77 & 1.34 & -7.58 & .66 & 88 & 080 & kout & & 1.2 & 9.0 & 5 & 0.8 & 8.3 & .07 & 0.3 & 8.6 & .01 \\
\hline 3.78 & -0.83 & 49.71 & & & 82 & & & 5.4 & 11.5 & & 4.7 & 10.5 & 16 & 4.1 & 10.6 & 0.12 \\
\hline 3.87 & .92 & 0.34 & & 5.06 & 57 & kout & & 5.5 & 11.5 & & 4.8 & 10.5 & 0.14 & 4.2 & 10.6 & 0.11 \\
\hline 108.89 & 0.32 & -52.61 & 50.00 & 5.29 & 050 & kout & & 5.7 & 11.7 & 7.02 & 5.0 & 10.7 & 5.40 & 4.4 & 10.7 & 4.19 \\
\hline 108.96 & -0.34 & -48.07 & 10.00 & 4.83 & 210 & kout & & 5.2 & 11.3 & 1.40 & 4.5 & 10.4 & 1.05 & 4.0 & 10.5 & 0.83 \\
\hline 108.97 & 0.31 & 56.09 & & 5.65 & 133 & kout & & 6.1 & 12.0 & 0.16 & 5.3 & 10.9 & 0.12 & 4.7 & 11.0 & 0.09 \\
\hline 109.02 & -0.25 & -47.12 & 0.90 & 4.73 & 0.109 & kout & hcs01 & 5.1 & 11.3 & 0.13 & 4.4 & 10.3 & 0.09 & 3.9 & 10.4 & 0.07 \\
\hline 109.11 & -0.86 & -48.82 & 2.60 & 4.89 & 0.315 & kout & hcs01 & 5.3 & 11.4 & 0.37 & 4.6 & 10.5 & 0.28 & 4.0 & 10.5 & 0.21 \\
\hline 109.32 & 0.25 & -55.48 & 3.80 & 5.57 & 460 & kout & hcs01 & 6.0 & 11.9 & 0.53 & 5.2 & 10.9 & 0.40 & 4.6 & 10.9 & 0.31 \\
\hline 109.35 & -0.37 & -56.85 & 1.00 & 5.71 & 121 & kout & hcs01 & 6.2 & 12.0 & 0.14 & 5.4 & 11.0 & 0.11 & 4.7 & 11.0 & 0.08 \\
\hline 109.37 & 0.39 & -53.93 & 0.87 & 5.40 & 0.105 & kout & hcs01 & 5.8 & 11.8 & 0.12 & 5.1 & 10.8 & 0.09 & 4.5 & 10.8 & 0.07 \\
\hline 109.50 & 6.50 & -9.20 & & 1.03 & 229 & kout & & 1.4 & 9.1 & 0.4 & 1.0 & 8.4 & 0.22 & 0.6 & 8.7 & 0.08 \\
\hline 109.56 & 1.62 & -52.63 & 1.50 & 5.26 & & kout & & 5.7 & 11.7 & 0.21 & 4.9 & 10.7 & 0.16 & 4.4 & 10.8 & 0.13 \\
\hline 109.62 & -1.34 & -48.13 & 1.10 & 4.80 & & kout & & 5.2 & 11.4 & 0.16 & 4.5 & 10.4 & 0.12 & 3.9 & 10.5 & 0.09 \\
\hline 109.64 & 1.94 & 56.86 & .60 & & & kout & & 6.2 & 12.1 & & 5.4 & 11.0 & 83 & 4.7 & 11.0 & 0.63 \\
\hline 9.79 & 1.99 & 50.65 & 2.00 & & & kout & & 6.6 & 12.4 & 0.28 & 5.7 & 11.3 & 0.21 & 5.1 & 11.3 & 0.17 \\
\hline 109.86 & -1.29 & 34.33 & & & & kout & & 3.8 & 10.4 & 0.2 & 3.2 & 9.6 & 0.17 & 2.7 & 9.8 & 0.12 \\
\hline 9.88 & & 1.29 & & & & kout & & 9.2 & 14.5 & 0 . & 8.1 & 13.2 & 14 & 7.2 & 12.9 & 0.11 \\
\hline 9.89 & 1.87 & -59.70 & & 6.00 & & kout & & 6.5 & 12.3 & 0.2 & 5.6 & 11.2 & 0.17 & 5.0 & 11.2 & 0.13 \\
\hline 98 & 3.05 & 65.17 & 1. & 6.6 & & ko & & 7.1 & 12.8 & 0. & 6.2 & 11.7 & 0.15 & 5.5 & 11.6 & 0.12 \\
\hline 96 & 0.46 & -53.91 & 4.80 & 5. & 581 & kout & & 5.8 & 11.8 & 0.6 & 5.0 & 10.8 & 0.50 & 4.4 & 10.9 & 0.39 \\
\hline 9.97 & -0.24 & 52.70 & 1.40 & 5.24 & 169 & kout & & 5.7 & 11.7 & 0.2 & 4.9 & 10.7 & 0.15 & 4.3 & 10.8 & 0.11 \\
\hline 109.99 & 0.87 & -52.39 & 3.10 & 5.21 & .375 & kout & hcs01 & 5.6 & 11.7 & 0.43 & 4.9 & 10.7 & 0.33 & 4.3 & 10.8 & 0.26 \\
\hline 110.00 & 0.00 & -52.10 & & 5.18 & 91.201 & kout & sod91 & 5.6 & 11.7 & 106.59 & 4.9 & 10.7 & 81.61 & 4.3 & 10.7 & 62.85 \\
\hline 110.05 & -0.17 & -50.46 & 66.00 & 5.00 & 986 & kout & & 5.4 & 11.5 & 9.31 & 4.7 & 10.6 & 7.06 & 4.1 & 10.6 & 5.37 \\
\hline 110.05 & 1.78 & -5.57 & 0.59 & 0.63 & 0.071 & kout & hes01 & 1.0 & 8.9 & 0.18 & 0.6 & 8.2 & 0.06 & 0.1 & 8.5 & \\
\hline 110.22 & 2.51 & -63.59 & 2.20 & 6.42 & 0.266 & kout & hcs01 & 6.9 & 12.7 & 0.31 & 6.0 & 11.6 & 0.23 & 5.3 & 11.5 & 0.18 \\
\hline 110.24 & 0.03 & -52.32 & 10.00 & 5.19 & 1.210 & kout & hcs01 & 5.6 & 11.7 & 1.41 & 4.9 & 10.7 & 1.08 & 4.3 & 10.8 & 0.83 \\
\hline
\end{tabular}


Table A.2. continued.

\begin{tabular}{|c|c|c|c|c|c|c|c|c|c|c|c|c|c|c|c|c|}
\hline $\begin{array}{r}l \\
\left({ }^{\circ}\right) \\
(1)\end{array}$ & $\begin{array}{l}b \\
\left({ }^{\circ}\right) \\
(2)\end{array}$ & $\begin{array}{r}V_{\text {lsr }} \\
\mathrm{km} \mathrm{s}^{-1} \\
\text { (3) }\end{array}$ & $\begin{array}{r}L_{\mathrm{CO}} \\
10^{3} \mathrm{~K} \mathrm{~km} \mathrm{~s}^{-1} \mathrm{pc}^{2} \\
(4)\end{array}$ & $\begin{array}{r}D \\
\mathrm{kpc} \\
(5)\end{array}$ & $\begin{array}{r}M_{\mathrm{GMCs}} \\
10^{5} M_{\odot} \\
(6)\end{array}$ & Mark & Ref. & $\begin{array}{r}D_{8.5} \\
\mathrm{kpc} \\
(9)\end{array}$ & $\begin{array}{l}R_{8.5} \\
\mathrm{kpc} \\
(10)\end{array}$ & $\begin{array}{r}M_{8.5} \\
10^{5} M_{\odot} \\
(11)\end{array}$ & $\begin{array}{l}D_{8.0} \\
\mathrm{kpc} \\
(12)\end{array}$ & $\begin{array}{l}R_{8.0} \\
\mathrm{kpc} \\
(13)\end{array}$ & $\begin{array}{r}M_{8.0} \\
10^{5} M_{\odot} \\
(14)\end{array}$ & $\begin{array}{l}D_{8.4} \\
\mathrm{kpc} \\
(15)\end{array}$ & $\begin{array}{l}R_{8.4} \\
\mathrm{kpc} \\
(16)\end{array}$ & $\begin{array}{r}M_{8.4} \\
10^{5} M_{\odot} \\
(17)\end{array}$ \\
\hline 10.26 & 1.06 & -53.87 & 1.10 & 5.35 & 0.133 & kout & hes01 & 5.8 & 11.8 & 0.16 & 5.0 & 10.8 & 0.12 & 4.4 & 10.9 & 0.09 \\
\hline 110.28 & -0.94 & -39.17 & 1.10 & 3.87 & 0.133 & kout & hcs01 & 4.3 & 10.7 & 0.16 & 3.6 & 9.9 & 0.12 & 3.1 & 10.0 & 0.09 \\
\hline 110.32 & 1.26 & -56.90 & 5.60 & 5.67 & 0.678 & kout & hcs01 & 6.1 & 12.1 & 0.78 & 5.3 & 11.0 & 0.59 & 4.7 & 11.0 & 0.47 \\
\hline 110.32 & 3.45 & -71.96 & 1.10 & 7.41 & 0.133 & kout & hes01 & 7.9 & 13.5 & 0.15 & 7.0 & 12.3 & 0.12 & 6.2 & 12.1 & 0.09 \\
\hline 110.34 & -0.63 & -36.62 & 0.72 & 3.62 & 0.087 & kout & hes01 & 4.0 & 10.6 & 0.11 & 3.4 & 9.7 & 0.08 & 2.9 & 9.9 & 0.06 \\
\hline 110.36 & 3.63 & -74.11 & 0.89 & 7.68 & 0.108 & kout & hes01 & 8.2 & 13.7 & 0.12 & 7.2 & 12.5 & 0.09 & 6.4 & 12.3 & 0.08 \\
\hline 110.40 & -0.51 & -45.50 & 72.00 & 4.49 & 8.712 & kout & hes01 & 4.9 & 11.2 & 10.38 & 4.2 & 10.3 & 7.62 & 3.7 & 10.4 & 5.92 \\
\hline 110.44 & 1.63 & -59.45 & 1.10 & 5.94 & 0.133 & kout & hes01 & 6.4 & 12.3 & 0.15 & 5.6 & 11.2 & 0.12 & 4.9 & 11.2 & 0.09 \\
\hline 110.45 & -1.65 & -48.80 & 1.10 & 4.82 & 0.133 & kout & hcs01 & 5.2 & 11.4 & 0.15 & 4.5 & 10.5 & 0.12 & 3.9 & 10.5 & 0.09 \\
\hline 110.51 & 1.03 & -51.80 & 0.87 & 5.12 & 0.105 & kout & hcs01 & 5.5 & 11.7 & 0.12 & 4.8 & 10.7 & 0.09 & 4.2 & 10.7 & 0.07 \\
\hline 110.56 & 0.18 & -45.90 & 0.86 & 4.52 & 0.104 & kout & hcs01 & 4.9 & 11.2 & 0.12 & 4.2 & 10.3 & 0.09 & 3.7 & 10.4 & 0.07 \\
\hline 110.58 & -0.08 & -53.52 & 5.40 & 5.29 & 0.653 & kout & hcs01 & 5.7 & 11.8 & 0.76 & 5.0 & 10.8 & 0.58 & 4.4 & 10.8 & 0.45 \\
\hline 110.60 & -2.16 & -37.41 & 1.40 & 3.69 & 0.169 & kout & hcs01 & 4.1 & 10.6 & 0.21 & 3.5 & 9.8 & 0.15 & 2.9 & 9.9 & 0.10 \\
\hline 110.64 & -0.92 & -48.08 & 3.10 & 4.73 & 0.375 & kout & hes01 & 5.1 & 11.4 & 0.44 & 4.4 & 10.4 & 0.32 & 3.9 & 10.5 & 0.25 \\
\hline 110.65 & -0.69 & -51.27 & 11.00 & 5.06 & 1.331 & kout & hcs01 & 5.5 & 11.6 & 1.57 & 4.8 & 10.6 & 1.20 & 4.2 & 10.7 & 0.92 \\
\hline 110.66 & -0.33 & -52.02 & 4.20 & 5.13 & 0.508 & kout & hcs01 & 5.6 & 11.7 & 0.61 & 4.8 & 10.7 & 0.44 & 4.2 & 10.7 & 0.34 \\
\hline 110.71 & 2.57 & -68.50 & 3.10 & 6.97 & 0.375 & kout & hcs01 & 7.5 & 13.2 & 0.43 & 6.5 & 12.0 & 0.33 & 5.8 & 11.9 & 0.26 \\
\hline 110.72 & -0.60 & -49.34 & 2.90 & 4.86 & 0.351 & kout & hcs01 & 5.3 & 11.5 & 0.42 & 4.6 & 10.5 & 0.31 & 4.0 & 10.6 & 0.24 \\
\hline 110.74 & 0.23 & -57.45 & 0.82 & 5.70 & 0.099 & kout & hes01 & 6.2 & 12.1 & 0.12 & 5.4 & 11.1 & 0.09 & 4.7 & 11.1 & 0.07 \\
\hline 110.77 & -2.71 & -34.75 & 5.60 & 3.43 & 0.678 & kout & hcs01 & 3.8 & 10.5 & 0.83 & 3.2 & 9.6 & 0.59 & 2.7 & 9.8 & 0.42 \\
\hline 110.77 & 1.89 & -52.98 & 0.76 & 5.23 & 0.092 & kout & hes01 & 5.7 & 11.8 & 0.11 & 4.9 & 10.8 & 0.08 & 4.3 & 10.8 & 0.06 \\
\hline 110.80 & 0.40 & -52.19 & 1.60 & 5.14 & 0.194 & kout & hes01 & 5.6 & 11.7 & 0.23 & 4.8 & 10.7 & 0.17 & 4.2 & 10.8 & 0.13 \\
\hline 110.88 & 1.88 & -10.46 & 13.00 & 1.11 & 1.573 & kout & hcs01 & 1.4 & 9.1 & 2.50 & 1.0 & 8.4 & 1.28 & 0.6 & 8.7 & 0.46 \\
\hline 110.89 & -0.29 & -55.63 & 1.40 & 5.50 & 0.169 & kout & hcs01 & 5.9 & 12.0 & 0.19 & 5.2 & 11.0 & 0.15 & 4.5 & 11.0 & 0.11 \\
\hline 110.93 & 1.59 & -50.65 & 0.77 & 4.98 & 0.093 & kout & hcs01 & 5.4 & 11.6 & 0.11 & 4.7 & 10.6 & 0.08 & 4.1 & 10.7 & 0.06 \\
\hline 110.94 & -0.04 & -52.05 & 0.79 & 5.12 & 0.096 & kout & $\mathrm{cs} 01$ & 5.6 & 11.7 & 0.11 & 4.8 & 10.7 & 0.08 & 4.2 & 10.8 & 0.06 \\
\hline 110.95 & 0.61 & -50.59 & 2.20 & 4.97 & 0.266 & kout & hcs01 & 5.4 & 11.6 & 0.31 & 4.7 & 10.6 & 0.24 & 4.1 & 10.7 & 0.18 \\
\hline 110.98 & -2.86 & -37.11 & 5.80 & 3.65 & 0.702 & kout & hes01 & 4.0 & 10.6 & 0.84 & 3.4 & 9.8 & 0.61 & 2.9 & 9.9 & 0.44 \\
\hline 110.99 & -0.99 & -45.10 & 0.70 & 4.42 & 0.085 & kout & hcs01 & 4.8 & 11.2 & 0.10 & 4.2 & 10.2 & 0.08 & 3.6 & 10.3 & 0.06 \\
\hline 111.02 & 2.42 & -47.11 & 1.00 & 4.62 & 0.121 & kout & hcs01 & 5.0 & 11.3 & 0.14 & 4.3 & 10.4 & 0.10 & 3.8 & 10.5 & 0.08 \\
\hline 111.03 & 3.01 & -40.30 & 0.77 & 3.95 & 0.093 & kout & hcs01 & 4.3 & 10.8 & 0.11 & 3.7 & 10.0 & 0.08 & 3.2 & 10.1 & 0.06 \\
\hline 111.09 & -2.34 & -47.82 & 2.70 & 4.69 & 0.327 & kout & hes01 & 5.1 & 11.4 & 0.39 & 4.4 & 10.4 & 0.29 & 3.8 & 10.5 & 0.21 \\
\hline 111.17 & -1.03 & -43.10 & 1.50 & 4.21 & 0.182 & kout & hes01 & 4.6 & 11.0 & 0.22 & 4.0 & 10.1 & 0.16 & 3.4 & 10.2 & 0.12 \\
\hline 111.19 & 0.60 & -49.22 & 0.87 & 4.82 & 0.105 & kout & hcs01 & 5.2 & 11.5 & 0.12 & 4.5 & 10.5 & 0.09 & 4.0 & 10.6 & 0.07 \\
\hline 111.20 & -2.25 & -52.54 & 0.91 & 5.17 & 0.110 & kout & hcs01 & 5.6 & 11.7 & 0.13 & 4.9 & 10.8 & 0.10 & 4.2 & 10.8 & 0.07 \\
\hline 111.20 & -0.84 & -52.98 & 1.10 & 5.21 & 0.133 & kout & hes01 & 5.6 & 11.8 & 0.15 & 4.9 & 10.8 & 0.12 & 4.3 & 10.8 & 0.09 \\
\hline 111.20 & 3.21 & -71.61 & 1.20 & 7.33 & 0.145 & kout & hcs01 & 7.9 & 13.5 & 0.17 & 6.9 & 12.3 & 0.13 & 6.1 & 12.1 & 0.10 \\
\hline 111.22 & -2.26 & -49.77 & 2.20 & 4.88 & 0.266 & kout & hcs01 & 5.3 & 11.5 & 0.31 & 4.6 & 10.6 & 0.24 & 4.0 & 10.6 & 0.18 \\
\hline 111.23 & 1.00 & -57.96 & 0.81 & 5.73 & 0.098 & kout & hcs01 & 6.2 & 12.2 & 0.11 & 5.4 & 11.1 & 0.09 & 4.8 & 11.1 & 0.07 \\
\hline 111.27 & 2.30 & -49.26 & 4.30 & 4.83 & 520 & kout & hes01 & 5.2 & 11.5 & 0.60 & 4.5 & 10.5 & 0.45 & 4.0 & 10.6 & 0.36 \\
\hline 111.28 & -1.44 & -39.84 & 15.00 & 3.89 & 815 & kout & hcs01 & 4.3 & 10.8 & 2.22 & 3.7 & 9.9 & 1.64 & 3.1 & 10.1 & 1.15 \\
\hline 1.30 & -0.59 & -48.54 & 80 & 4.74 & 0.339 & kout & 01 & 5.2 & 11.4 & 41 & 4.5 & 10.5 & 0.31 & 3.9 & 10.5 & 0.23 \\
\hline 111.32 & -0.68 & -45.00 & 9.70 & 4.39 & 174 & kout & & 4.8 & 11.2 & .40 & 4.1 & 10.2 & 1.02 & 3.6 & 10.3 & 0.79 \\
\hline 111.32 & 3.36 & -65.79 & 1.20 & 6.63 & 0.145 & kout & hcs01 & 7.1 & 12.9 & 0.17 & 6.2 & 11.8 & 0.13 & 5.5 & 11.7 & 0.10 \\
\hline 111.37 & 0.46 & -50.58 & 3.60 & 4.95 & 0.436 & kout & hes01 & 5.4 & 11.6 & 0.52 & 4.7 & 10.6 & 0.39 & 4.1 & 10.7 & 0.30 \\
\hline 111.38 & -1.55 & -36.27 & 0.69 & 3.54 & 0.083 & kout & hcs01 & 3.9 & 10.6 & 0.10 & 3.3 & 9.7 & 0.07 & 2.8 & 9.9 & 0.05 \\
\hline 111.40 & -2.60 & -36.77 & 1.50 & 3.59 & 0.182 & kout & hes01 & 4.0 & 10.6 & 0.23 & 3.4 & 9.8 & 0.16 & 2.8 & 9.9 & 0.11 \\
\hline 111.43 & -2.64 & -46.59 & 1.40 & 4.55 & 0.169 & kout & hcs01 & 5.0 & 11.3 & 0.20 & 4.3 & 10.4 & 0.15 & 3.7 & 10.4 & 0.11 \\
\hline 111.44 & 1.13 & -49.67 & 1.00 & 4.85 & 0.121 & kout & hcs01 & 5.3 & 11.5 & 0.14 & 4.6 & 10.6 & 0.11 & 4.0 & 10.6 & 0.08 \\
\hline 111.45 & 1.23 & -47.21 & 1.20 & 4.60 & 0.145 & kout & hes01 & 5.0 & 11.3 & 0.17 & 4.3 & 10.4 & 0.13 & 3.8 & 10.5 & 0.10 \\
\hline 111.49 & 0.73 & -53.81 & 200.00 & 5.28 & 24.200 & kout & hcs01 & 5.7 & 11.9 & 28.20 & 5.0 & 10.8 & 21.70 & 4.4 & 10.9 & 16.81 \\
\hline 111.49 & 1.28 & -46.17 & 3.60 & 4.50 & 0.436 & kout & hcs01 & 4.9 & 11.3 & 0.52 & 4.2 & 10.3 & 0.38 & 3.7 & 10.4 & 0.29 \\
\hline 111.50 & -3.00 & -41.70 & & 4.07 & 27.542 & kout & sod91 & 4.4 & 10.9 & 32.19 & 3.8 & 10.0 & 24.01 & 3.2 & 10.2 & 17.03 \\
\hline 111.50 & 2.00 & -10.50 & & 1.10 & 5.370 & kout & sod91 & 1.4 & 9.1 & 8.70 & 1.0 & 8.4 & 4.44 & 0.6 & 8.7 & 1.60 \\
\hline 111.57 & -2.73 & -39.52 & 33.00 & 3.85 & 3.993 & kout & hcs01 & 4.2 & 10.8 & 4.75 & 3.6 & 9.9 & 3.49 & 3.1 & 10.0 & 2.59 \\
\hline 111.61 & -2.69 & -37.91 & 1.90 & 3.69 & 0.230 & kout & hcs01 & 4.1 & 10.7 & 0.28 & 3.5 & 9.8 & 0.21 & 2.9 & 10.0 & 0.14 \\
\hline 111.64 & 1.32 & -43.43 & 0.89 & 4.22 & 0.108 & kout & hes & 4.6 & 11.1 & 0.13 & 4.0 & 10.2 & 0.10 & 3.4 & 10.3 & 0.07 \\
\hline 111.67 & -2.29 & -47.11 & 5.00 & 4.59 & 0.605 & kout & hes & 5.0 & 11.3 & 0.72 & 4.3 & 10.4 & 0.53 & 3.7 & 10.5 & 0.39 \\
\hline 111.68 & 3.17 & -68.98 & 1.60 & 6.99 & 0.194 & kout & & 7.5 & 13.3 & 0.22 & 6.6 & 12.1 & 0.17 & 5.8 & 11.9 & 0.13 \\
\hline 111.70 & 0.01 & -30.14 & 1.10 & 2.94 & 0.133 & kout & & 3.3 & 10.2 & 0.17 & 2.8 & 9.4 & 0.12 & 2.3 & 9.6 & 0.08 \\
\hline .70 & 2.61 & -56.17 & 3.00 & 5.53 & 0.363 & kout & hcs01 & 6.0 & 12.1 & 0.43 & 5.2 & 11.0 & 0.32 & 4.6 & 11.0 & 0.25 \\
\hline & 4.20 & -29.53 & 1.60 & 2.90 & 0.194 & kout & & 3.2 & 10.2 & 0.24 & 2.7 & 9.4 & 0.17 & 2.3 & 9.6 & 0.12 \\
\hline 111.71 & 2.48 & -54.70 & 4.60 & 5.37 & 0.557 & kout & & 5.8 & 11.9 & 0.65 & 5.0 & 10.9 & 0.48 & 4.4 & 11.0 & 0.37 \\
\hline 111.84 & 1.87 & -51.99 & 0.83 & 5.08 & 0.100 & kout & hcs01 & 5.5 & 11.7 & 0.12 & 4.8 & 10.7 & 0.09 & 4.2 & 10.8 & 0.07 \\
\hline
\end{tabular}


Table A.2. continued.

\begin{tabular}{|c|c|c|c|c|c|c|c|c|c|c|c|c|c|c|c|c|}
\hline $\begin{array}{l}l \\
\left({ }^{\circ}\right) \\
(1)\end{array}$ & $\begin{array}{l}b \\
\left({ }^{\circ}\right) \\
(2)\end{array}$ & $\begin{array}{r}V_{\text {lsr }} \\
\mathrm{km} \mathrm{s}^{-1} \\
\text { (3) }\end{array}$ & $\begin{array}{r}L_{\mathrm{CO}} \\
10^{3} \mathrm{~K} \mathrm{~km} \mathrm{~s}^{-1} \mathrm{pc}^{2} \\
(4)\end{array}$ & $\begin{array}{r}D \\
\mathrm{kpc} \\
(5)\end{array}$ & $\begin{array}{r}M_{\mathrm{GMCs}} \\
10^{5} M_{\odot} \\
\text { (6) }\end{array}$ & Mark & Ref. & $\begin{array}{r}D_{8.5} \\
\mathrm{kpc} \\
(9)\end{array}$ & $\begin{array}{l}R_{8.5} \\
\mathrm{kpc} \\
(10)\end{array}$ & $\begin{array}{r}M_{8.5} \\
10^{5} M_{\odot} \\
(11)\end{array}$ & $\begin{array}{l}D_{8.0} \\
\text { kpc } \\
(12)\end{array}$ & $\begin{array}{l}R_{8.0} \\
\mathrm{kpc} \\
(13)\end{array}$ & $\begin{array}{r}M_{8.0} \\
10^{5} M_{\odot} \\
(14)\end{array}$ & $\begin{array}{l}D_{8.4} \\
\mathrm{kpc} \\
(15)\end{array}$ & $\begin{array}{l}R_{8.4} \\
\mathrm{kpc} \\
(16)\end{array}$ & $\begin{array}{r}M_{8.4} \\
10^{5} M_{\odot} \\
(17)\end{array}$ \\
\hline 111.89 & -2.73 & -36.90 & 1.00 & 3.58 & 0.121 & kout & hes01 & 3.9 & 10.6 & 0.14 & 3.4 & 9.8 & 0.11 & 2.8 & 9.9 & 0.07 \\
\hline 111.90 & 2.77 & -78.40 & 3.10 & 8.18 & 0.375 & kout & hcs01 & 8.8 & 14.3 & 0.43 & 7.7 & 13.0 & 0.33 & 6.8 & 12.7 & 0.26 \\
\hline 111.92 & 0.93 & -50.11 & 17.00 & 4.87 & 2.057 & kout & hcs01 & 5.3 & 11.6 & 2.44 & 4.6 & 10.6 & 1.84 & 4.0 & 10.7 & 1.39 \\
\hline 112.05 & 2.35 & -46.01 & 0.92 & 4.46 & 0.111 & kout & hcs01 & 4.9 & 11.3 & 0.13 & 4.2 & 10.3 & 0.10 & 3.7 & 10.4 & 0.08 \\
\hline 112.07 & 2.14 & -52.67 & 0.73 & 5.14 & 0.088 & kout & hcs01 & 5.6 & 11.8 & 0.10 & 4.8 & 10.8 & 0.08 & 4.2 & 10.8 & 0.06 \\
\hline 112.09 & 2.75 & -54.64 & 1.20 & 5.35 & 0.145 & kout & hcs01 & 5.8 & 11.9 & 0.17 & 5.0 & 10.9 & 0.13 & 4.4 & 11.0 & 0.10 \\
\hline 112.13 & 2.39 & -78.74 & 1.10 & 8.21 & 0.133 & kout & hcs01 & 8.8 & 14.4 & 0.15 & 7.7 & 13.0 & 0.12 & 6.8 & 12.7 & 0.09 \\
\hline 112.17 & -2.30 & -37.65 & 20.00 & 3.64 & 2.420 & kout & hcs01 & 4.0 & 10.7 & 2.92 & 3.4 & 9.8 & 2.11 & 2.9 & 10.0 & 1.54 \\
\hline 112.18 & -1.86 & -45.14 & 6.50 & 4.36 & 0.787 & kout & hcs01 & 4.8 & 11.2 & 0.95 & 4.1 & 10.3 & 0.70 & 3.5 & 10.4 & 0.51 \\
\hline 112.20 & 5.21 & -52.70 & 0.89 & 5.17 & 0.108 & kout & hcs01 & 5.6 & 11.8 & 0.13 & 4.8 & 10.8 & 0.09 & 4.3 & 10.9 & 0.07 \\
\hline 112.22 & -2.54 & -44.53 & 0.77 & 4.31 & 0.093 & kout & hcs01 & 4.7 & 11.2 & 0.11 & 4.0 & 10.2 & 0.08 & 3.5 & 10.3 & 0.06 \\
\hline 112.27 & -1.08 & -42.56 & 0.94 & 4.10 & 0.114 & kout & hcs01 & 4.5 & 11.0 & 0.14 & 3.9 & 10.1 & 0.10 & 3.3 & 10.2 & 0.07 \\
\hline 112.31 & 2.50 & -78.14 & 0.77 & 8.13 & 0.093 & kout & hcs01 & 8.7 & 14.3 & 0.11 & 7.6 & 13.0 & 0.08 & 6.7 & 12.7 & 0.06 \\
\hline 112.62 & -0.78 & -33.14 & 0.71 & 3.18 & 0.086 & kout & hcs01 & 3.5 & 10.4 & 0.10 & 3.0 & 9.6 & 0.08 & 2.5 & 9.7 & 0.05 \\
\hline 112.73 & 2.70 & -16.19 & 6.80 & 1.60 & 0.823 & kout & hcs01 & 1.9 & 9.4 & 1.16 & 1.5 & 8.7 & 0.72 & 1.1 & 9.0 & 0.39 \\
\hline 112.86 & -0.69 & -35.33 & 2.10 & 3.38 & 0.254 & kout & hcs01 & 3.7 & 10.5 & 0.30 & 3.2 & 9.7 & 0.23 & 2.7 & 9.8 & 0.16 \\
\hline 112.90 & 0.34 & -52.42 & 0.93 & 5.07 & 0.113 & kout & hcs01 & 5.5 & 11.8 & 0.13 & 4.8 & 10.8 & 0.10 & 4.2 & 10.8 & 0.08 \\
\hline 112.99 & -2.18 & -34.98 & 1.20 & 3.34 & 0.145 & kout & hcs01 & 3.7 & 10.5 & 0.18 & 3.1 & 9.7 & 0.12 & 2.6 & 9.8 & 0.09 \\
\hline 113.03 & -1.52 & -30.52 & 0.99 & 2.92 & 0.120 & kout & hcs01 & 3.3 & 10.2 & 0.15 & 2.7 & 9.4 & 0.10 & 2.2 & 9.6 & 0.07 \\
\hline 113.46 & 0.94 & -59.37 & 0.87 & 5.80 & 0.105 & kout & hcs01 & 6.3 & 12.4 & 0.12 & 5.4 & 11.3 & 0.09 & 4.8 & 11.3 & 0.07 \\
\hline 113.49 & 1.61 & -12.09 & 0.96 & 1.19 & 0.116 & kout & hcs01 & 1.5 & 9.2 & 0.18 & 1.1 & 8.5 & 0.10 & 0.7 & 8.8 & 0.04 \\
\hline 113.60 & -0.90 & -49.25 & 2.10 & 4.71 & 0.254 & kout & hcs01 & 5.1 & 11.6 & 0.30 & 4.4 & 10.6 & 0.22 & 3.8 & 10.6 & 0.17 \\
\hline 113.70 & -0.62 & -43.19 & 1.80 & 4.10 & 0.218 & kout & hes01 & 4.5 & 11.1 & 0.26 & 3.9 & 10.2 & 0.20 & 3.3 & 10.3 & 0.14 \\
\hline 113.87 & -0.19 & -38.59 & 1.10 & 3.64 & 0.133 & kout & hcs01 & 4.0 & 10.8 & 0.16 & 3.4 & 9.9 & 0.12 & 2.9 & 10.0 & 0.08 \\
\hline 114.09 & -0.58 & -36.19 & 1.00 & 3.41 & 0.121 & kout & hcs01 & 3.8 & 10.6 & 0.15 & 3.2 & 9.8 & 0.11 & 2.7 & 9.9 & 0.08 \\
\hline 114.12 & -1.15 & -32.95 & 0.69 & 3.10 & 0.083 & kout & hes01 & 3.5 & 10.4 & 0.11 & 2.9 & 9.6 & 0.07 & 2.4 & 9.7 & 0.05 \\
\hline 114.16 & 2.04 & -65.99 & 1.30 & 6.54 & 0.157 & kout & hes01 & 7.0 & 13.1 & 0.18 & 6.1 & 11.9 & 0.14 & 5.4 & 11.8 & 0.11 \\
\hline 114.20 & 2.66 & -51.63 & 1.40 & 4.95 & 0.169 & kout & hcs01 & 5.4 & 11.8 & 0.20 & 4.6 & 10.8 & 0.15 & 4.1 & 10.8 & 0.12 \\
\hline 114.21 & -0.98 & -31.87 & 0.91 & 3.00 & 0.110 & kout & hes01 & 3.3 & 10.3 & 0.13 & 2.8 & 9.5 & 0.10 & 2.3 & 9.7 & 0.06 \\
\hline 114.24 & 0.33 & -53.87 & 1.50 & 5.17 & 0.182 & kout & hcs01 & 5.6 & 12.0 & 0.21 & 4.9 & 10.9 & 0.16 & 4.2 & 10.9 & 0.12 \\
\hline 114.33 & 0.79 & -101.15 & & 12.35 & 0.140 & kout & bw94 & 12.7 & 17.9 & 0.15 & 11.1 & 16.1 & 0.11 & 9.6 & 15.2 & 0.08 \\
\hline 114.41 & 3.21 & -69.82 & 0.77 & 7.01 & 0.093 & kout & hes01 & 7.5 & 13.5 & 0.11 & 6.6 & 12.3 & 0.08 & 5.8 & 12.1 & 0.06 \\
\hline 114.47 & -0.89 & -46.94 & 0.77 & 4.44 & 0.093 & kout & hes01 & 4.8 & 11.4 & 0.11 & 4.2 & 10.4 & 0.08 & 3.6 & 10.5 & 0.06 \\
\hline 114.48 & 2.22 & -64.34 & 0.78 & 6.34 & 0.094 & kout & hes01 & 6.8 & 12.9 & 0.11 & 6.0 & 11.8 & 0.08 & 5.2 & 11.7 & 0.06 \\
\hline 114.54 & 2.88 & -57.11 & 0.87 & 5.52 & 0.105 & kout & hcs01 & 6.0 & 12.2 & 0.12 & 5.2 & 11.2 & 0.09 & 4.6 & 11.2 & 0.07 \\
\hline 114.56 & 2.91 & -72.09 & 0.97 & 7.30 & 0.117 & kout & hes01 & 7.8 & 13.7 & 0.13 & 6.8 & 12.5 & 0.10 & 6.0 & 12.3 & 0.08 \\
\hline 114.57 & -0.19 & -37.56 & 1.30 & 3.52 & 0.157 & kout & hes01 & 3.9 & 10.7 & 0.19 & 3.3 & 9.8 & 0.14 & 2.8 & 10.0 & 0.10 \\
\hline 114.58 & -0.42 & -49.68 & 10.00 & 4.72 & 1.210 & kout & hes01 & 5.1 & 11.6 & 1.41 & 4.4 & 10.6 & 1.05 & 3.8 & 10.7 & 0.78 \\
\hline 114.89 & 3.81 & -22.32 & 0.92 & 2.10 & 0.111 & kout & hes01 & 2.4 & 9.8 & 0.14 & 2.0 & 9.0 & 0.10 & 1.6 & 9.3 & 0.06 \\
\hline 114.93 & 3.18 & -64.42 & 1.10 & 6.35 & 0.133 & kout & hes01 & 6.8 & 13.0 & 0.15 & 6.0 & 11.8 & 0.12 & 5.2 & 11.7 & 0.09 \\
\hline 114.98 & 4.88 & -18.27 & 0.60 & 1.73 & 0.073 & kout & hcs01 & 2.0 & 9.5 & 0.10 & 1.6 & 8.8 & 0.06 & 1.2 & 9.1 & 0.04 \\
\hline 115.02 & 1.05 & -13.06 & 1.40 & 1.24 & 0.169 & kout & hcs01 & 1.5 & 9.3 & 0.25 & 1.2 & 8.6 & 0.16 & 0.8 & 8.8 & 0.07 \\
\hline 115.14 & -1.51 & -40.54 & 3.40 & 3.79 & 0.411 & kout & hcs01 & 4.2 & 10.9 & 0.50 & 3.6 & 10.0 & 0.37 & 3.0 & 10.2 & 0.26 \\
\hline 115.26 & 3.62 & -60.99 & 1.00 & 5.95 & 0.121 & kout & hcs01 & 6.4 & 12.6 & 0.14 & 5.6 & 11.5 & 0.11 & 4.9 & 11.5 & 0.08 \\
\hline 115.38 & 2.06 & -10.95 & 1.30 & 1.04 & 0.157 & kout & hes01 & 1.3 & 9.2 & 0.25 & 1.0 & 8.5 & 0.15 & 0.6 & 8.8 & 0.05 \\
\hline 115.58 & 3.97 & -24.83 & 9.40 & 2.31 & 1.137 & kout & hes01 & 2.6 & 9.9 & 1.44 & 2.2 & 9.1 & 1.03 & 1.8 & 9.4 & 0.69 \\
\hline 115.71 & -1.66 & -40.16 & 6.00 & 3.73 & 0.726 & kout & hcs01 & 4.1 & 10.9 & 0.88 & 3.5 & 10.0 & 0.64 & 3.0 & 10.1 & 0.47 \\
\hline 115.82 & 3.81 & -67.34 & 1.10 & 6.69 & 0.133 & kout & hcs01 & 7.2 & 13.3 & 0.15 & 6.3 & 12.1 & 0.12 & 5.5 & 12.0 & 0.09 \\
\hline 116.02 & 1.65 & -8.08 & 1.10 & 0.77 & 0.133 & kout & hcs01 & 1.1 & 9.0 & 0.27 & 0.7 & 8.3 & 0.11 & 0.3 & 8.6 & 0.02 \\
\hline 116.07 & 4.19 & -68.23 & 0.77 & 6.81 & 0.093 & kout & hes01 & 7.3 & 13.4 & 0.11 & 6.4 & 12.2 & 0.08 & 5.6 & 12.1 & 0.06 \\
\hline 116.14 & -1.63 & -42.87 & 1.10 & 3.98 & 0.133 & kout & hes01 & 4.4 & 11.1 & 0.16 & 3.7 & 10.2 & 0.11 & 3.2 & 10.3 & 0.09 \\
\hline 116.16 & -0.67 & -45.04 & 1.10 & 4.19 & 0.133 & kout & hcs01 & 4.6 & 11.3 & 0.16 & 3.9 & 10.4 & 0.12 & 3.4 & 10.4 & 0.09 \\
\hline 116.34 & 0.65 & -45.85 & 1.10 & 4.27 & 0.133 & kout & hes01 & 4.7 & 11.4 & 0.16 & 4.0 & 10.4 & 0.12 & 3.5 & 10.5 & 0.09 \\
\hline 116.58 & -2.97 & -36.61 & 1.10 & 3.37 & 0.133 & kout & hes01 & 3.7 & 10.7 & 0.16 & 3.2 & 9.8 & 0.12 & 2.6 & 10.0 & 0.08 \\
\hline 116.94 & -2.24 & -44.51 & 4.10 & 4.12 & 0.496 & kout & hcs01 & 4.5 & 11.3 & 0.59 & 3.9 & 10.3 & 0.44 & 3.3 & 10.4 & 0.32 \\
\hline 117.10 & -2.42 & -37.92 & 0.80 & 3.47 & 0.097 & kout & hes01 & 3.8 & 10.8 & 0.12 & 3.3 & 9.9 & 0.09 & 2.7 & 10.0 & 0.06 \\
\hline 117.15 & 4.78 & -7.47 & 4.70 & 0.70 & 0.569 & kout & hes01 & 1.0 & 9.0 & 1.16 & 0.7 & 8.3 & 0.57 & 0.3 & 8.6 & 0.10 \\
\hline 117.19 & 5.06 & -73.29 & 1.20 & 7.48 & 0.145 & kout & hes01 & 8.0 & 14.1 & 0.17 & 7.0 & 12.8 & 0.13 & 6.1 & 12.6 & 0.10 \\
\hline 117.26 & -0.26 & -48.23 & 1.40 & 4.49 & 0.169 & kout & hes & 4.9 & 11.6 & 0.20 & 4.2 & 10.6 & 0.15 & 3.6 & 10.7 & 0.11 \\
\hline 117.43 & 1.12 & -61.56 & 1.30 & 5.94 & 0.157 & kout & hcs01 & 6.4 & 12.8 & 0.18 & 5.6 & 11.7 & 0.14 & 4.9 & 11.6 & 0.11 \\
\hline 117.60 & 2.32 & -45.17 & 0.99 & 4.17 & 0.120 & kout & hcs01 & 4.6 & 11.4 & 0.15 & 3.9 & 10.4 & 0.10 & 3.4 & 10.5 & 0.08 \\
\hline 117.61 & 0.00 & -46.51 & 1.80 & 4.30 & 0.218 & kout & hes01 & 4.7 & 11.5 & 0.26 & 4.0 & 10.5 & 0.19 & 3.5 & 10.6 & 0.14 \\
\hline 117.72 & 3.15 & -17.43 & 7.70 & 1.58 & 0.932 & kout & hcs01 & 1.9 & 9.5 & 1.35 & 1.5 & 8.8 & 0.84 & 1.1 & 9.1 & 0.45 \\
\hline 118.00 & 5.00 & -14.02 & 1.30 & 1.27 & 0.157 & kout & hes 01 & 1.6 & 9.3 & 0.25 & 1.2 & 8.6 & 0.14 & 0.8 & 8.9 & 0.06 \\
\hline
\end{tabular}


Table A.2. continued.

\begin{tabular}{|c|c|c|c|c|c|c|c|c|c|c|c|c|c|c|c|c|}
\hline $\begin{array}{r}l \\
\left({ }^{\circ}\right) \\
(1) \\
\end{array}$ & $\begin{array}{r}b \\
\left({ }^{\circ}\right) \\
(2) \\
\end{array}$ & $\begin{array}{r}V_{\mathrm{lsr}} \\
\mathrm{km} \mathrm{s}^{-1} \\
(3)\end{array}$ & $\begin{array}{r}L_{\mathrm{CO}} \\
10^{3} \mathrm{~K} \mathrm{~km} \mathrm{~s}^{-1} \mathrm{pc}^{2} \\
(4) \\
\end{array}$ & $\begin{array}{r}D \\
\mathrm{kpc} \\
(5) \\
\end{array}$ & $\begin{array}{r}M_{\mathrm{GMCs}} \\
10^{5} M_{\odot} \\
(6) \\
\end{array}$ & Mark & $\overline{\text { Ref. }}$ & $\begin{array}{r}D_{8.5} \\
\mathrm{kpc} \\
(9) \\
\end{array}$ & $\begin{array}{l}R_{8.5} \\
\mathrm{kpc} \\
(10)\end{array}$ & $\begin{array}{r}M_{8.5} \\
10^{5} M_{\odot} \\
(11) \\
\end{array}$ & $\begin{array}{l}D_{8.0} \\
\mathrm{kpc} \\
(12) \\
\end{array}$ & $\begin{array}{l}R_{8.0} \\
\mathrm{kpc} \\
(13)\end{array}$ & $\begin{array}{r}M_{8.0} \\
10^{5} M_{\odot} \\
(14)\end{array}$ & $\begin{array}{l}D_{8.4} \\
\text { kpc } \\
(15) \\
\end{array}$ & $\begin{array}{l}R_{8.4} \\
\mathrm{kpc} \\
(16)\end{array}$ & $\begin{array}{r}M_{8.4} \\
10^{5} M_{\odot} \\
(17)\end{array}$ \\
\hline 118.12 & 1.58 & -92.50 & 0.83 & 10.41 & 0.100 & kout & hcs01 & 11.2 & 16.9 & 0.12 & 9.8 & 15.3 & 0.09 & 8.4 & 14.5 & 0.07 \\
\hline 118.46 & 1.20 & -61.22 & 1.00 & 5.89 & 0.121 & kout & hcs01 & 6.4 & 12.8 & 0.14 & 5.5 & 11.7 & 0.11 & 4.8 & 11.6 & 0.08 \\
\hline 118.57 & 3.62 & -5.59 & 0.77 & 0.51 & 0.093 & kout & hcs01 & 0.8 & 8.9 & 0.23 & 0.5 & 8.2 & 0.09 & 0.1 & 8.6 & \\
\hline 118.62 & 2.78 & -63.24 & 1.40 & 6.15 & 0.169 & kout & hcs01 & 6.6 & 13.0 & 0.19 & 5.8 & 11.9 & 0.15 & 5.1 & 11.8 & 0.12 \\
\hline 118.66 & -0.38 & -50.16 & 0.84 & 4.66 & 0.102 & kout & hcs01 & 5.1 & 11.8 & 0.12 & 4.4 & 10.8 & 0.09 & 3.8 & 10.8 & 0.07 \\
\hline 118.68 & -1.33 & -39.03 & 1.20 & 3.53 & 0.145 & kout & hcs01 & 3.9 & 10.9 & 0.18 & 3.3 & 10.0 & 0.13 & 2.8 & 10.1 & 0.09 \\
\hline 118.73 & 2.90 & -67.77 & 0.92 & 6.71 & 0.111 & kout & hes01 & 7.2 & 13.6 & 0.13 & 6.3 & 12.3 & 0.10 & 5.5 & 12.2 & 0.07 \\
\hline 118.77 & 3.13 & -69.33 & 1.60 & 6.92 & 0.194 & kout & hcs 01 & 7.4 & 13.7 & 0.22 & 6.5 & 12.5 & 0.17 & 5.7 & 12.3 & 0.13 \\
\hline 118.90 & 3.19 & -20.17 & 1.90 & 1.79 & 0.230 & kout & hcs01 & 2.1 & 9.7 & 0.32 & 1.7 & 8.9 & 0.21 & 1.3 & 9.2 & 0.12 \\
\hline 119.18 & -0.89 & -32.94 & 0.78 & 2.94 & 0.094 & kout & hes01 & 3.3 & 10.5 & 0.12 & 2.8 & 9.7 & 0.09 & 2.3 & 9.8 & 0.06 \\
\hline 119.22 & 1.71 & -62.46 & 1.40 & 6.04 & 0.169 & kout & hcs01 & 6.5 & 13.0 & 0.20 & 5.7 & 11.9 & 0.15 & 5.0 & 11.7 & 0.12 \\
\hline 119.41 & 1.53 & -61.60 & 1.60 & 5.93 & 0.194 & kout & $\operatorname{cs} 01$ & 6.4 & 12.9 & 0.23 & 5.6 & 11.8 & 0.17 & 4.9 & 11.7 & 0.13 \\
\hline 119.42 & 0.74 & -53.88 & 1.70 & 5.05 & 0.206 & kout & $\mathrm{cs} 01$ & 5.5 & 12.2 & 0.24 & 4.7 & 11.1 & 0.18 & 4.1 & 11.1 & 0.14 \\
\hline 119.51 & 0.39 & -50.22 & 0.86 & 4.65 & 0.104 & kout & $\mathrm{cs} 01$ & 5.1 & 11.8 & 0.13 & 4.4 & 10.8 & 0.09 & 3.8 & 10.9 & 0.07 \\
\hline 119.58 & 3.22 & -46.78 & 2.10 & 4.30 & 0.254 & kout & hcs01 & 4.7 & 11.6 & 0.30 & 4.0 & 10.6 & 0.22 & 3.5 & 10.7 & 0.17 \\
\hline 119.60 & 1.25 & -61.49 & 0.73 & 5.92 & 0.088 & kout & hcs01 & 6.4 & 12.9 & 0.10 & 5.6 & 11.8 & 0.08 & 4.9 & 11.7 & 0.06 \\
\hline 119.64 & -0.50 & -50.36 & 1.60 & 4.66 & 0.194 & kout & hes01 & 5.1 & 11.9 & 0.23 & 4.4 & 10.9 & 0.17 & 3.8 & 10.9 & 0.13 \\
\hline 119.92 & 1.95 & -68.14 & 1.00 & 6.75 & 0.121 & kout & hes01 & 7.3 & 13.7 & 0.14 & 6.3 & 12.4 & 0.11 & 5.5 & 12.2 & 0.08 \\
\hline 120.12 & -0.18 & -49.32 & 1.20 & 4.54 & 0.145 & kout & hcs01 & 5.0 & 11.8 & 0.18 & 4.3 & 10.8 & 0.13 & 3.7 & 10.8 & 0.10 \\
\hline 120.13 & 1.45 & -65.26 & 1.20 & 6.38 & 0.145 & kout & hcs01 & 6.9 & 13.4 & 0.17 & 6.0 & 12.2 & 0.13 & 5.2 & 12.0 & 0.10 \\
\hline 120.13 & 3.46 & -68.99 & 8.00 & 6.89 & 0.968 & kout & hcs 01 & 7.4 & 13.8 & 1.12 & 6.5 & 12.6 & 0.86 & 5.7 & 12.3 & 0.66 \\
\hline 120.26 & 2.89 & -18.98 & 8.40 & 1.66 & 1.016 & kout & hcs01 & 1.9 & 9.6 & 1.33 & 1.6 & 8.9 & 0.94 & 1.2 & 9.2 & 0.53 \\
\hline 120.28 & 2.12 & -50.03 & 3.30 & 4.62 & 0.399 & kout & & 5.0 & 11.9 & 0.47 & 4.3 & 10.9 & 0.35 & 3.8 & 10.9 & 0.27 \\
\hline 120.30 & 2.85 & -67.89 & 1.00 & 6.74 & 121 & kout & & 7.3 & 13.7 & 0.14 & 6.3 & 12.5 & 11 & 5.5 & 12.3 & 0.08 \\
\hline 120.39 & 0.13 & -48.06 & 1.80 & 4.41 & 0.218 & kout & & 4.8 & 11.7 & 0.26 & 4.1 & 10.7 & 19 & 3.6 & 10.8 & 0.15 \\
\hline 120.42 & 3.63 & -71.72 & 0.89 & 7.26 & 08 & ut & & 7.8 & 14.2 & .12 & 6.8 & 12.9 & 09 & 6.0 & 12.6 & 0.07 \\
\hline 120.48 & 1.11 & -60.01 & 3.70 & 5.74 & 448 & kout & & 6.2 & 12.8 & 0.52 & 5.4 & 11.7 & 0.40 & 4.7 & 11.6 & 0.30 \\
\hline 120.49 & 1.78 & -62.67 & 4.90 & 6.07 & 0.593 & kout & & 6.6 & 13.1 & 0.70 & 5.7 & 11.9 & 0.52 & 5.0 & 11.8 & 0.40 \\
\hline 120.52 & 3.65 & -38.62 & 1.50 & 3.47 & 0.182 & kout & hcs01 & 3.8 & 10.9 & 0.22 & 3.3 & 10.0 & 0.16 & 2.8 & 10.2 & 0.12 \\
\hline 120.70 & 4.20 & -11.55 & 1.10 & 1.01 & 0.133 & kout & hcs01 & 1.3 & 9.2 & 0.22 & 0.9 & 8.5 & 0.11 & 0.6 & 8.8 & 0.05 \\
\hline 120.77 & 4.98 & -9.58 & 0.63 & 0.84 & 0.076 & kout & hes01 & 1.1 & 9.1 & 0.13 & 0.8 & 8.4 & 0.07 & 0.5 & 8.7 & 0.03 \\
\hline 120.79 & -0.38 & -46.18 & 3.30 & 4.21 & 0.399 & kout & hcs01 & 4.6 & 11.6 & 0.48 & 4.0 & 10.6 & 0.36 & 3.4 & 10.6 & 0.26 \\
\hline 120.80 & 1.10 & -63.70 & & 6.20 & 1.100 & kout & $\mathrm{mk} 88$ & 6.7 & 13.2 & 1.28 & 5.8 & 12.1 & 0.96 & 5.1 & 11.9 & 0.74 \\
\hline 120.87 & 1.08 & -64.45 & 3.40 & 6.29 & 0.411 & kout & hcs01 & 6.8 & 13.3 & 0.48 & 5.9 & 12.1 & 0.36 & 5.1 & 12.0 & 0.27 \\
\hline 120.93 & 1.15 & -62.64 & 11.00 & 6.06 & 1.331 & kout & & 6.6 & 13.1 & 1.58 & 5.7 & 12.0 & 1.18 & 5.0 & 11.8 & 0.91 \\
\hline 1.02 & -1.39 & -19.65 & 1.50 & 1.70 & 182 & kout & & 2.0 & 9.7 & .25 & 1.6 & 8.9 & 16 & 1.2 & 9.2 & .09 \\
\hline 121.05 & -0.22 & -47.23 & 96 & 4.31 & 116 & kout & & 4.7 & 11.7 & .14 & 4.1 & 10.7 & 10 & 3.5 & 10.7 & 0.08 \\
\hline 121.46 & 0.78 & -59.76 & 20 & 5.71 & 145 & kout & & 6.2 & 12.9 & 17 & 5.4 & 11.7 & 13 & 4.7 & 11.6 & 0.10 \\
\hline .52 & 00 & -67.98 & 85 & 6.77 & 103 & out & & 7.3 & 13.8 & 12 & .4 & 12.6 & 99 & .6 & 12.3 & 0.07 \\
\hline 121.54 & 3.85 & -40.17 & 0.78 & 3.60 & 094 & kout & & 4.0 & 11.1 & .12 & 3.4 & 10.2 & 0.08 & 2.9 & 10.3 & 0.06 \\
\hline 121.69 & -0.94 & -44.70 & 0.85 & 4.04 & 103 & kout & & 4.4 & 11.5 & 0.12 & 3.8 & 10.5 & 0.09 & 3.2 & 10.6 & 0.06 \\
\hline 121.96 & 4.10 & -67.94 & 1.70 & 6.79 & 206 & kout & hes01 & 7.3 & 13.8 & 0.24 & 6.4 & 12.6 & 0.18 & 5.6 & 12.4 & 0.14 \\
\hline 122.00 & 0.00 & -17.60 & & 1.51 & 175 & kout & & 1.8 & 9.6 & 1.67 & 1.4 & 8.8 & 1.01 & 1.0 & 9.1 & 0.52 \\
\hline 122.00 & 3.68 & -5.06 & 1.30 & 0.43 & 0.157 & kout & hes01 & 0.7 & 8.9 & 0.42 & 0.4 & 8.2 & 0.14 & 0.1 & 8.5 & 0.01 \\
\hline 122.13 & 3.18 & -61.28 & 1.00 & 5.92 & 0.121 & kout & hes 01 & 6.4 & 13.1 & 0.14 & 5.6 & 11.9 & 0.11 & 4.8 & 11.8 & 0.08 \\
\hline 122.14 & -1.37 & -13.85 & 1.40 & 1.18 & 0.169 & kout & hes01 & 1.5 & 9.4 & 0.27 & 1.1 & 8.6 & 0.15 & 0.7 & 8.9 & 0.06 \\
\hline 122.18 & 3.00 & -60.12 & 0.71 & 5.78 & 0.086 & kout & hes01 & 6.3 & 13.0 & 0.10 & 5.4 & 11.8 & 0.08 & 4.7 & 11.7 & 0.06 \\
\hline 122.20 & -0.87 & -42.30 & 1.20 & 3.79 & 0.145 & kout & hcs01 & 4.2 & 11.3 & 0.18 & 3.6 & 10.3 & 0.13 & 3.0 & 10.4 & 0.09 \\
\hline 122.22 & 2.70 & -60.53 & 1.10 & 5.83 & 133 & kout & hcs01 & 6.3 & 13.0 & 0.16 & 5.5 & 11.9 & 0.12 & 4.8 & 11.8 & 0.09 \\
\hline 122.26 & .08 & -68.55 & .70 & 6.88 & 206 & kout & & 7.4 & 13.9 & 0.24 & 6.5 & 12.7 & 0.18 & 5.6 & 12.5 & 0.14 \\
\hline 122.45 & 2.62 & -55.17 & .20 & 5.19 & 145 & kout & & 5.6 & 12.5 & .17 & 4.9 & 11.4 & 0.13 & 4.2 & 11.4 & 0.09 \\
\hline 122.52 & 1.66 & -58.86 & .72 & 5.62 & & kout & & 6.1 & 12.8 & 10 & 5.3 & 11.7 & 0.08 & 4.6 & 11.6 & 0.06 \\
\hline .53 & 1.5 & -57.22 & 2.10 & 5.42 & & kout & & 5.9 & 12.7 & 0.30 & 5.1 & 11.6 & 0.22 & 4.4 & 11.5 & 0.17 \\
\hline .56 & & -96.25 & 0.89 & 11.60 & & kol & & 12.5 & 18.5 & .13 & 10.9 & 16.6 & 0.10 & 9.2 & 15.6 & 0.07 \\
\hline 122.59 & -0.62 & -19.09 & 11.00 & & & kout & & 1.9 & 9.7 & & 1.5 & 8.9 & 1.13 & 1.1 & 9.2 & 0.61 \\
\hline 122.63 & & -66.57 & & 6.60 & & kout & & 7.1 & 13.7 & 0.70 & 6.2 & 12.5 & 0.53 & 5.4 & 12.3 & 0.41 \\
\hline 122.80 & 2.89 & -63.95 & 2.40 & 6.27 & & ko & & 6.8 & 13.4 & 0.34 & 5.9 & 12.2 & 0.26 & 5.1 & 12.1 & 0.19 \\
\hline 122.90 & 1.36 & -55.75 & 0.72 & 5.25 & 0.087 & kout & & 5.7 & 12.5 & 0.10 & 4.9 & 11.5 & 0.08 & 4.3 & 11.4 & 0.06 \\
\hline 122.96 & & -10.27 & 1.40 & 0.87 & 0.169 & kout & hcs 01 & 1.1 & 9.2 & 0.27 & 0.8 & 8.5 & 0.14 & 0.5 & 8.8 & 0.06 \\
\hline 123.06 & -6.31 & -30.40 & 1.10 & 2.20 & 0.133 & stel & $\operatorname{css} 90$ & & & 0.13 & & & 0.13 & & & 0.13 \\
\hline 123.06 & -0.78 & -44.77 & 21.00 & 4.04 & 2.541 & kout & hes01 & 4.4 & 11.5 & 3.01 & 3.8 & 10.6 & 2.25 & 3.2 & 10.6 & 1.59 \\
\hline 123.24 & 3.07 & -63.79 & 1.40 & 6.26 & 0.169 & kout & hcs01 & 6.8 & 13.5 & 0.20 & 5.9 & 12.2 & 0.15 & 5.1 & 12.1 & 0.11 \\
\hline 123.45 & 3.21 & -61.50 & 9.20 & 5.97 & 1.113 & kout & hcs01 & 6.5 & 13.2 & 1.32 & 5.6 & 12.0 & 0.98 & 4.9 & 11.9 & 0.75 \\
\hline 123.50 & 2.00 & -66.40 & & 6.40 & 0.110 & kout & $\mathrm{mk} 88$ & 7.1 & 13.8 & 0.14 & 6.2 & 12.5 & 0.10 & 5.4 & 12.3 & 0.08 \\
\hline 123.62 & -1.36 & -21.23 & 0.96 & 1.80 & 0.116 & kout & hcs01 & 2.1 & 9.8 & 0.16 & 1.7 & 9.0 & 0.10 & 1.3 & 9.3 & 0.06 \\
\hline
\end{tabular}


Table A.2. continued.

\begin{tabular}{|c|c|c|c|c|c|c|c|c|c|c|c|c|c|c|c|c|}
\hline $\begin{array}{r}l \\
\left({ }^{\circ}\right) \\
(1)\end{array}$ & $\begin{array}{l}b \\
\left({ }^{\circ}\right) \\
(2)\end{array}$ & $\begin{array}{r}V_{\mathrm{lsr}} \\
\mathrm{km} \mathrm{s}^{-1} \\
\text { (3) }\end{array}$ & $\begin{array}{r}L_{\mathrm{CO}} \\
10^{3} \mathrm{~K} \mathrm{~km} \mathrm{~s}^{-1} \mathrm{pc}^{2} \\
(4)\end{array}$ & $\begin{array}{r}D \\
\mathrm{kpc} \\
(5)\end{array}$ & $\begin{array}{r}M_{\mathrm{GMCs}} \\
10^{5} M_{\odot} \\
(6)\end{array}$ & Mark & Ref. & $\begin{array}{l}D_{8.5} \\
\mathrm{kpc} \\
(9)\end{array}$ & $\begin{array}{l}R_{8.5} \\
\mathrm{kpc} \\
(10)\end{array}$ & $\begin{array}{r}M_{8.5} \\
10^{5} M_{\odot} \\
(11)\end{array}$ & $\begin{array}{l}D_{8.0} \\
\mathrm{kpc} \\
(12)\end{array}$ & $\begin{array}{l}R_{8.0} \\
\mathrm{kpc} \\
(13)\end{array}$ & $\begin{array}{r}M_{8.0} \\
10^{5} M_{\odot} \\
(14)\end{array}$ & $\begin{array}{l}D_{8.4} \\
\mathrm{kpc} \\
(15)\end{array}$ & $\begin{array}{l}R_{8.4} \\
\mathrm{kpc} \\
(16)\end{array}$ & $\begin{array}{r}M_{8.4} \\
10^{5} M_{\odot} \\
(17)\end{array}$ \\
\hline 123.68 & 2.86 & -63.72 & 1.80 & 6.26 & 0.218 & kout & hcs01 & 6.8 & 13.5 & 0.26 & 5.9 & 12.3 & 0.19 & 5.1 & 12.1 & 0.14 \\
\hline 123.94 & -0.12 & -44.03 & 2.30 & 3.96 & 0.278 & kout & hes01 & 4.3 & 11.5 & 0.33 & 3.7 & 10.5 & 0.24 & 3.2 & 10.6 & 0.18 \\
\hline 124.01 & 1.93 & -51.53 & 0.78 & 4.78 & 0.094 & kout & hes01 & 5.2 & 12.2 & 0.11 & 4.5 & 11.1 & 0.08 & 3.9 & 11.1 & 0.06 \\
\hline 124.12 & -0.18 & -44.61 & 1.20 & 4.02 & 0.145 & kout & hcs 01 & 4.4 & 11.6 & 0.17 & 3.8 & 10.6 & 0.13 & 3.2 & 10.7 & 0.09 \\
\hline 124.26 & -0.17 & -46.45 & 0.87 & 4.21 & 0.105 & kout & hcs01 & 4.6 & 11.7 & 0.13 & 4.0 & 10.7 & 0.09 & 3.4 & 10.8 & 0.07 \\
\hline 124.28 & 0.22 & -43.59 & 1.70 & 3.91 & 0.206 & kout & hes01 & 4.3 & 11.5 & 0.25 & 3.7 & 10.5 & 0.18 & 3.1 & 10.6 & 0.13 \\
\hline 124.33 & 0.50 & -46.07 & 1.70 & 4.17 & 0.206 & kout & hes 01 & 4.6 & 11.7 & 0.25 & 3.9 & 10.7 & 0.18 & 3.4 & 10.8 & 0.14 \\
\hline 124.39 & 1.98 & -71.98 & 2.60 & 7.42 & 0.315 & kout & hcs01 & 8.0 & 14.6 & 0.37 & 7.0 & 13.3 & 0.28 & 6.0 & 12.9 & 0.21 \\
\hline 124.60 & 2.54 & -86.91 & & 10.43 & 0.520 & kout & bw94 & 10.7 & 17.1 & 0.55 & 9.4 & 15.4 & 0.42 & 8.0 & 14.6 & 0.31 \\
\hline 124.60 & 3.15 & -11.01 & 0.97 & 0.92 & 0.117 & kout & hcs01 & 1.2 & 9.2 & 0.20 & 0.9 & 8.5 & 0.11 & 0.5 & 8.8 & 0.03 \\
\hline 124.61 & 2.50 & -87.43 & 4.70 & 10.07 & 0.569 & kout & hcs01 & 10.8 & 17.2 & 0.65 & 9.5 & 15.5 & 0.51 & 8.1 & 14.7 & 0.37 \\
\hline 124.78 & 2.93 & -61.85 & 1.10 & 6.05 & 0.133 & kout & hcs01 & 6.5 & 13.4 & 0.15 & 5.7 & 12.2 & 0.12 & 4.9 & 12.0 & 0.09 \\
\hline 124.94 & -0.16 & -42.01 & 0.75 & 3.75 & 0.091 & kout & hcs01 & 4.1 & 11.4 & 0.11 & 3.5 & 10.4 & 0.08 & 3.0 & 10.5 & 0.06 \\
\hline 125.08 & 0.78 & -67.75 & 2.50 & 6.84 & 0.303 & kout & hcs01 & 7.4 & 14.1 & 0.35 & 6.4 & 12.8 & 0.27 & 5.6 & 12.5 & 0.20 \\
\hline 125.29 & 0.58 & -65.85 & 1.20 & 6.58 & 0.145 & kout & hcs01 & 7.1 & 13.9 & 0.17 & 6.2 & 12.6 & 0.13 & 5.4 & 12.4 & 0.10 \\
\hline 125.58 & 2.07 & -53.78 & 4.90 & 5.06 & 0.593 & kout & hcs01 & 5.5 & 12.5 & 0.70 & 4.7 & 11.4 & 0.51 & 4.1 & 11.4 & 0.39 \\
\hline 125.78 & 1.66 & -65.47 & 3.50 & 6.55 & 0.424 & kout & hcs01 & 7.1 & 13.9 & 0.50 & 6.2 & 12.6 & 0.38 & 5.3 & 12.4 & 0.28 \\
\hline 126.03 & 4.37 & -7.92 & 0.79 & 0.65 & 0.096 & kout & hcs01 & 0.9 & 9.1 & 0.18 & 0.6 & 8.4 & 0.08 & 0.3 & 8.7 & 0.02 \\
\hline 126.40 & -0.79 & -11.86 & 9.70 & 0.97 & 1.174 & kout & hes 01 & 1.2 & 9.3 & 1.80 & 0.9 & 8.6 & 1.01 & 0.6 & 8.9 & 0.45 \\
\hline 126.43 & -0.24 & -57.89 & 1.50 & 5.57 & 0.182 & kout & hcs01 & 6.0 & 13.0 & 0.21 & 5.2 & 11.9 & 0.16 & 4.5 & 11.8 & 0.12 \\
\hline 127.00 & -1.00 & -11.80 & & 0.96 & 4.365 & kout & sod91 & 1.2 & 9.3 & 6.82 & 0.9 & 8.6 & 3.84 & 0.6 & 8.8 & 1.71 \\
\hline 127.52 & 1.86 & -80.95 & 1.40 & 9.19 & 0.169 & kout & hcs01 & 9.9 & 16.5 & 0.20 & 8.6 & 14.9 & 0.15 & 7.4 & 14.3 & 0.11 \\
\hline 127.70 & -0.11 & -49.78 & 0.82 & 4.62 & 0.099 & kout & hes01 & 5.0 & 12.3 & 0.12 & 4.3 & 11.2 & 0.09 & 3.7 & 11.2 & 0.06 \\
\hline 127.87 & 1.17 & -59.33 & 0.79 & 5.81 & 0.096 & kout & hes 01 & 6.3 & 13.3 & 0.11 & 5.5 & 12.1 & 0.09 & 4.7 & 12.0 & 0.06 \\
\hline 128.30 & 1.75 & -48.24 & 0.94 & 4.47 & 0.114 & kout & hcs 01 & 4.9 & 12.1 & 0.14 & 4.2 & 11.1 & 0.10 & 3.6 & 11.1 & 0.07 \\
\hline 128.55 & -0.27 & -14.33 & 4.00 & 1.17 & 0.484 & kout & hcs01 & 1.4 & 9.4 & 0.69 & 1.1 & 8.7 & 0.43 & 0.7 & 9.0 & 0.17 \\
\hline 128.62 & 1.22 & -51.83 & 0.92 & 4.90 & 0.111 & kout & hcs01 & 5.3 & 12.5 & 0.13 & 4.6 & 11.4 & 0.10 & 4.0 & 11.4 & 0.07 \\
\hline 128.66 & 1.45 & -54.30 & 0.88 & 5.20 & 0.106 & kout & hes 01 & 5.7 & 12.8 & 0.13 & 4.9 & 11.7 & 0.09 & 4.2 & 11.6 & 0.07 \\
\hline 128.76 & 1.94 & -82.04 & 3.30 & 9.57 & 0.399 & kout & hcs01 & 10.3 & 17.0 & 0.46 & 9.0 & 15.3 & 0.35 & 7.7 & 14.6 & 0.26 \\
\hline 128.81 & 1.33 & -83.16 & 1.60 & 9.80 & 0.194 & kout & hcs01 & 10.6 & 17.2 & 0.23 & 9.2 & 15.5 & 0.17 & 7.8 & 14.7 & 0.12 \\
\hline 128.82 & 0.06 & -32.89 & 1.60 & 2.84 & 0.194 & kout & hcs01 & 3.2 & 10.8 & 0.25 & 2.7 & 9.9 & 0.18 & 2.2 & 10.0 & 0.12 \\
\hline 128.99 & -0.16 & -36.78 & 0.90 & 3.23 & 0.109 & kout & hcs 01 & 3.6 & 11.1 & 0.14 & 3.0 & 10.2 & 0.09 & 2.5 & 10.3 & 0.07 \\
\hline 129.16 & 0.62 & -38.81 & 0.73 & 3.44 & 0.088 & kout & hcs01 & 3.8 & 11.3 & 0.11 & 3.2 & 10.4 & 0.08 & 2.7 & 10.4 & 0.05 \\
\hline 129.20 & -0.37 & -37.55 & 1.00 & 3.31 & 0.121 & kout & hcs01 & 3.7 & 11.2 & 0.15 & 3.1 & 10.3 & 0.11 & 2.6 & 10.4 & 0.07 \\
\hline 129.39 & -0.26 & -34.45 & 0.81 & 3.00 & 0.098 & kout & hcs01 & 3.3 & 10.9 & 0.12 & 2.8 & 10.0 & 0.09 & 2.3 & 10.2 & 0.06 \\
\hline 130.07 & 5.08 & -14.09 & 0.64 & 1.15 & 0.077 & kout & hcs01 & 1.4 & 9.5 & 0.11 & 1.1 & 8.7 & 0.07 & 0.8 & 9.0 & 0.04 \\
\hline 130.59 & 1.83 & -44.61 & 3.10 & 4.11 & 0.375 & kout & hcs01 & 4.5 & 11.9 & 0.45 & 3.9 & 10.9 & 0.34 & 3.3 & 10.9 & 0.24 \\
\hline 130.66 & 0.22 & -60.08 & 0.75 & 6.07 & 0.091 & kout & hcs01 & 6.6 & 13.7 & 0.11 & 5.7 & 12.5 & 0.08 & 4.9 & 12.3 & 0.06 \\
\hline 131.79 & -0.70 & -53.83 & 2.30 & 5.29 & 0.278 & kout & hcs01 & 5.8 & 13.1 & 0.33 & 5.0 & 11.9 & 0.25 & 4.2 & 11.8 & 0.18 \\
\hline 131.86 & 1.33 & -78.76 & 0.76 & 9.43 & 0.092 & kout & hcs01 & 10.2 & 17.1 & 0.11 & 8.9 & 15.4 & 0.08 & 7.5 & 14.6 & 0.06 \\
\hline 132.19 & -0.88 & -55.40 & 3.80 & 5.52 & 0.460 & kout & hcs01 & 6.0 & 13.3 & 0.54 & 5.2 & 12.1 & 0.41 & 4.4 & 11.9 & 0.29 \\
\hline 132.54 & -1.62 & -37.55 & 0.84 & 3.38 & 0.102 & kout & hcs01 & 3.7 & 11.4 & 0.12 & 3.2 & 10.4 & 0.09 & 2.7 & 10.5 & 0.07 \\
\hline 133.00 & 0.90 & -43.00 & 6.22 & 2.50 & 0.260 & stel & dlp +96 & & & 0.26 & & & 0.26 & & & 0.26 \\
\hline 133.29 & 0.81 & -48.12 & 0.89 & 4.63 & 0.108 & kout & $\mathrm{h} c \mathrm{~s} 01$ & 5.1 & 12.5 & 0.13 & 4.4 & 11.4 & 0.10 & 3.7 & 11.4 & 0.07 \\
\hline 133.37 & -0.08 & -15.57 & 1.50 & 1.27 & 0.182 & kout & hcs01 & 1.5 & 9.6 & 0.25 & 1.2 & 8.9 & 0.16 & 0.9 & 9.1 & 0.09 \\
\hline 133.37 & 1.25 & -40.68 & 0.90 & 3.74 & 0.109 & kout & hcs01 & 4.1 & 11.7 & 0.13 & 3.5 & 10.7 & 0.10 & 3.0 & 10.8 & 0.07 \\
\hline 133.39 & 1.01 & -42.43 & 44.00 & 3.95 & 5.324 & kout & hcs01 & 4.3 & 11.9 & 6.31 & 3.7 & 10.9 & 4.67 & 3.2 & 10.9 & 3.49 \\
\hline 133.41 & 0.25 & -50.53 & 19.00 & 4.95 & 2.299 & kout & hes 01 & 5.4 & 12.8 & 2.74 & 4.6 & 11.7 & 1.99 & 4.0 & 11.6 & 1.50 \\
\hline 133.47 & 0.02 & -48.27 & 1.80 & 4.66 & 0.218 & kout & hcs 01 & 5.1 & 12.6 & 0.26 & 4.4 & 11.5 & 0.19 & 3.7 & 11.4 & 0.14 \\
\hline 133.50 & 9.00 & -15.70 & & 1.31 & 2.884 & kout & sod91 & 1.5 & 9.6 & 3.78 & 1.2 & 8.9 & 2.42 & 1.0 & 9.2 & 1.68 \\
\hline 133.67 & 0.62 & -44.55 & 2.80 & 4.21 & 0.339 & kout & hcs01 & 4.6 & 12.1 & 0.40 & 4.0 & 11.1 & 0.31 & 3.4 & 11.1 & 0.22 \\
\hline 133.70 & 1.20 & -40.90 & 7.97 & 2.50 & 0.330 & stel & dlp+96 & & & 0.33 & & & 0.33 & & & 0.33 \\
\hline 133.79 & 1.26 & -39.31 & 1.50 & 3.60 & 0.182 & kout & hcs01 & 4.0 & 11.6 & 0.22 & 3.4 & 10.6 & 0.16 & 2.9 & 10.7 & 0.12 \\
\hline 133.90 & 0.40 & -47.60 & 7.84 & 2.50 & 0.330 & stel & dlp +96 & & & 0.33 & & & 0.33 & & & 0.33 \\
\hline 133.90 & 1.31 & -45.17 & 0.86 & 4.30 & 0.104 & kout & hes01 & 4.7 & 12.2 & 0.12 & 4.0 & 11.2 & 0.09 & 3.5 & 11.2 & 0.07 \\
\hline 134.01 & 0.65 & -48.42 & 45.00 & 4.71 & 5.445 & kout & hcs01 & 5.1 & 12.6 & 6.38 & 4.4 & 11.5 & 4.75 & 3.8 & 11.5 & 3.54 \\
\hline 134.01 & 0.60 & -46.59 & 1.20 & 4.48 & 0.145 & kout & hcs01 & 4.9 & 12.4 & 0.17 & 4.2 & 11.3 & 0.13 & 3.6 & 11.3 & 0.09 \\
\hline 134.10 & 0.80 & -48.40 & 7.04 & 2.50 & 0.290 & stel & dlp+96 & & & 0.29 & & & 0.29 & & & 0.29 \\
\hline 134.29 & -1.85 & -49.09 & 1.60 & 4.82 & 0.194 & kout & hcs01 & 5.3 & 12.7 & 0.23 & 4.5 & 11.6 & 0.17 & 3.8 & 11.5 & 0.12 \\
\hline 134.42 & -1.62 & -47.62 & 1.00 & 4.63 & 0.121 & kout & hcs01 & 5.1 & 12.6 & 0.15 & 4.4 & 11.5 & 0.11 & 3.7 & 11.4 & 0.08 \\
\hline 134.45 & -0.14 & -13.81 & 1.80 & 1.12 & 0.218 & kout & hcs01 & 1.4 & 9.5 & 0.34 & 1.1 & 8.8 & 0.21 & 0.7 & 9.0 & 0.09 \\
\hline 34.48 & 2.20 & -30.92 & 0.68 & 2.72 & 0.082 & kout & hcs01 & 3.0 & 10.8 & 0.10 & 2.6 & 10.0 & 0.07 & 2.1 & 10.1 & 0.05 \\
\hline 134.72 & -0.68 & -42.90 & 3.10 & 4.06 & 0.375 & kout & hcs01 & 4.4 & 12.1 & 0.44 & 3.8 & 11.0 & 0.33 & 3.2 & 11.0 & 0.23 \\
\hline 134.84 & 1.41 & -40.94 & 0.76 & 3.83 & 0.092 & kout & hes01 & 4.2 & 11.8 & 0.11 & 3.6 & 10.8 & 0.08 & 3.1 & 10.9 & 0.06 \\
\hline 134.91 & 1.59 & -39.22 & 0.69 & 3.63 & 0.083 & kout & hes01 & 4.0 & 11.7 & 0.10 & 3.4 & 10.7 & 0.07 & 2.9 & 10.7 & 0.05 \\
\hline
\end{tabular}


Table A.2. continued.

\begin{tabular}{|c|c|c|c|c|c|c|c|c|c|c|c|c|c|c|c|c|}
\hline $\begin{array}{l}l \\
\left({ }^{\circ}\right) \\
(1)\end{array}$ & $\begin{array}{r}b \\
\left({ }^{\circ}\right) \\
(2)\end{array}$ & $\begin{array}{r}V_{\mathrm{lsr}} \\
\mathrm{km} \mathrm{s}^{-1} \\
\text { (3) }\end{array}$ & $\begin{array}{r}L_{\mathrm{CO}} \\
10^{3} \mathrm{~K} \mathrm{~km} \mathrm{~s}^{-1} \mathrm{pc}^{2} \\
(4)\end{array}$ & $\begin{array}{r}D \\
\mathrm{kpc} \\
(5)\end{array}$ & $\begin{array}{r}M_{\mathrm{GMCs}} \\
10^{5} M_{\odot} \\
(6)\end{array}$ & Mark & Ref. & $\begin{array}{r}D_{8.5} \\
\mathrm{kpc} \\
(9)\end{array}$ & $\begin{array}{l}R_{8.5} \\
\mathrm{kpc} \\
(10)\end{array}$ & $\begin{array}{r}M_{8.5} \\
10^{5} M_{\odot} \\
(11)\end{array}$ & $\begin{array}{l}D_{8.0} \\
\mathrm{kpc} \\
(12)\end{array}$ & $\begin{array}{l}R_{8.0} \\
\mathrm{kpc} \\
(13)\end{array}$ & $\begin{array}{r}M_{8.0} \\
10^{5} M_{\odot} \\
(14)\end{array}$ & $\begin{array}{l}D_{8.4} \\
\mathrm{kpc} \\
(15)\end{array}$ & $\begin{array}{l}R_{8.4} \\
\mathrm{kpc} \\
(16)\end{array}$ & $\begin{array}{r}M_{8.4} \\
10^{5} M_{\odot} \\
(17)\end{array}$ \\
\hline 135.16 & -0.06 & -45.02 & 2.10 & 4.34 & 0.254 & kout & hes01 & 4.7 & 12.3 & 0.30 & 4.1 & 11.3 & 0.23 & 3.5 & 11.2 & 0.17 \\
\hline 135.24 & -0.38 & -41.78 & 1.30 & 3.95 & 0.157 & kout & hcs01 & 4.3 & 12.0 & 0.19 & 3.7 & 11.0 & 0.14 & 3.1 & 11.0 & 0.10 \\
\hline 135.27 & 1.06 & -45.42 & 1.90 & 4.40 & 0.230 & kout & hcs01 & 4.8 & 12.4 & 0.27 & 4.1 & 11.3 & 0.20 & 3.5 & 11.3 & 0.15 \\
\hline 136.30 & 0.19 & -57.26 & 1.70 & 6.16 & 0.206 & kout & hes01 & 6.7 & 14.1 & 0.24 & 5.8 & 12.8 & 0.18 & 4.9 & 12.5 & 0.13 \\
\hline 136.34 & -1.72 & -10.78 & 1.20 & 0.87 & 0.145 & kout & hcs01 & 1.1 & 9.3 & 0.23 & 0.8 & 8.6 & 0.12 & 0.5 & 8.9 & 0.05 \\
\hline 136.34 & 0.85 & -74.35 & 1.30 & 9.44 & 0.157 & kout & hcs01 & 10.2 & 17.4 & 0.18 & 8.9 & 15.7 & 0.14 & 7.5 & 14.8 & 0.10 \\
\hline 136.50 & 1.20 & -36.40 & 4.40 & 2.50 & 0.180 & stel & dlp+96 & & & 0.18 & & & 0.18 & & & 0.18 \\
\hline 136.62 & 0.34 & -56.80 & 0.94 & 6.13 & 0.114 & kout & hcs01 & 6.7 & 14.1 & 0.14 & 5.8 & 12.8 & 0.10 & 4.9 & 12.5 & 0.07 \\
\hline 136.70 & 1.23 & -36.88 & 15.00 & 3.44 & 1.815 & kout & hcs01 & 3.8 & 11.6 & 2.21 & 3.2 & 10.6 & 1.57 & 2.7 & 10.7 & 1.12 \\
\hline 136.79 & 0.44 & -59.57 & 1.70 & 6.61 & 0.206 & kout & hcs01 & 7.2 & 14.6 & 0.24 & 6.2 & 13.2 & 0.18 & 5.3 & 12.9 & 0.13 \\
\hline 136.88 & 0.26 & -56.82 & 1.30 & 6.16 & 0.157 & kout & hcs01 & 6.7 & 14.1 & 0.19 & 5.8 & 12.9 & 0.14 & 4.9 & 12.6 & 0.10 \\
\hline 136.92 & 1.16 & -40.47 & 8.70 & 3.87 & 1.053 & kout & hcs01 & 4.3 & 12.0 & 1.30 & 3.6 & 10.9 & 0.91 & 3.1 & 11.0 & 0.68 \\
\hline 137.00 & 1.30 & -38.50 & 9.19 & 2.50 & 0.380 & stel & dlp+96 & & & 0.38 & & & 0.38 & & & 0.38 \\
\hline 137.15 & 3.13 & -50.96 & 0.92 & 5.31 & 0.111 & kout & hcs01 & 5.8 & 13.3 & 0.13 & 5.0 & 12.1 & 0.10 & 4.3 & 12.0 & 0.07 \\
\hline 137.22 & 1.36 & -40.13 & 0.92 & 3.85 & 0.111 & kout & hcs01 & 4.2 & 12.0 & 0.13 & 3.6 & 10.9 & 0.10 & 3.1 & 11.0 & 0.07 \\
\hline 137.35 & 1.80 & -38.36 & 1.20 & 3.64 & 0.145 & kout & hcs01 & 4.0 & 11.8 & 0.18 & 3.4 & 10.8 & 0.13 & 2.9 & 10.8 & 0.09 \\
\hline 137.70 & 1.50 & -38.90 & 3.86 & 2.50 & 0.160 & stel & dlp+96 & & & 0.16 & & & 0.16 & & & 0.16 \\
\hline 137.72 & -0.71 & -45.39 & 0.81 & 4.56 & 0.098 & kout & hcs01 & 5.0 & 12.6 & 0.12 & 4.3 & 11.5 & 0.09 & 3.6 & 11.4 & 0.06 \\
\hline 137.72 & 4.18 & -9.85 & 0.93 & 0.80 & 0.113 & kout & hcs01 & 1.0 & 9.3 & 0.18 & 0.8 & 8.6 & 0.11 & 0.5 & 8.9 & 0.04 \\
\hline 137.76 & -0.97 & -103.73 & 1.60 & 21.60 & 0.194 & kout & hcs01 & 23.5 & 30.4 & 0.23 & 20.3 & 26.8 & 0.17 & 15. & 22. & 0.09 \\
\hline 137.95 & 1.72 & -42.72 & 0.83 & 4.22 & 0.100 & kout & hcs01 & 4.6 & 12.3 & 0.12 & 4.0 & 11.3 & 0.09 & 3.4 & 11.2 & 0.06 \\
\hline 138.00 & 4.00 & -9.80 & & 0.80 & 0.170 & kout & sod91 & 1.0 & 9.3 & 0.27 & 0.7 & 8.6 & 0.13 & 0.5 & 8.9 & 0.07 \\
\hline 138.00 & 2.50 & -11.80 & & 0.97 & 0.224 & kout & sod91 & 1.2 & 9.4 & 0.34 & 0.9 & 8.7 & 0.19 & 0.6 & 9.0 & 0.09 \\
\hline 138.29 & 2.42 & -12.60 & 1.20 & 1.04 & 0.145 & kout & hcs01 & 1.3 & 9.5 & 0.23 & 1.0 & 8.8 & 0.13 & 0.7 & 9.0 & 0.07 \\
\hline 138.38 & -2.14 & -46.63 & 1.20 & 4.79 & 0.145 & kout & hcs01 & 5.2 & 12.9 & 0.17 & 4.5 & 11.7 & 0.13 & 3.8 & 11.6 & 0.09 \\
\hline 138.50 & 1.60 & -38.70 & 4.84 & 2.50 & 0.200 & stel & dlp+96 & & & 0.20 & & & 0.20 & & & 0.20 \\
\hline 138.52 & -0.81 & -45.75 & 2.50 & 4.67 & 0.303 & kout & hcs01 & 5.1 & 12.8 & 0.36 & 4.4 & 11.7 & 0.27 & 3.7 & 11.5 & 0.19 \\
\hline 138.58 & 1.74 & -37.92 & 0.98 & 3.66 & 0.119 & kout & hcs01 & 4.0 & 11.8 & 0.14 & 3.4 & 10.8 & 0.10 & 2.9 & 10.9 & 0.07 \\
\hline 8.66 & 5.16 & -15.38 & 1.20 & 1.30 & 0.145 & kout & hcs01 & 1.5 & 9.7 & 0.19 & 1.2 & 9.0 & 0.12 & 0.9 & 9.2 & 0.07 \\
\hline 9.20 & 1.00 & -39.60 & 1.71 & 2.50 & 0.071 & stel & dlp+96 & & & 0.07 & & & 0.07 & & & 0.07 \\
\hline 139.22 & 2.71 & -51.19 & 0.73 & 5.56 & 0.088 & kout & hcs01 & 6.0 & 13.7 & 0.10 & 5.2 & 12.4 & 0.08 & 4.5 & 12.2 & 0.06 \\
\hline 139.24 & -2.57 & -13.87 & 0.79 & 1.16 & 0.096 & kout & hes01 & 1.4 & 9.6 & 0.14 & 1.1 & 8.9 & 0.09 & 0.8 & 9.1 & 0.05 \\
\hline 139.24 & 1.01 & -39.86 & 1.90 & 3.94 & 0.230 & kout & hcs01 & 4.3 & 12.1 & 0.27 & 3.7 & 11.1 & 0.20 & 3.1 & 11.1 & 0.14 \\
\hline 139.31 & -2.05 & -48.73 & 4.00 & 5.18 & 0.484 & kout & hcs01 & 5.6 & 13.3 & 0.57 & 4.9 & 12.1 & 0.43 & 4.1 & 11.9 & 0.30 \\
\hline 139.36 & 2.87 & -53.02 & 1.00 & 5.87 & 0.121 & kout & hcs01 & 6.4 & 14.0 & 0.14 & 5.5 & 12.7 & 0.11 & 4.7 & 12.5 & 0.08 \\
\hline 139.41 & -0.40 & -37.07 & 0.92 & 3.59 & 0.111 & kout & hcs01 & 4.0 & 11.8 & 0.14 & 3.4 & 10.8 & 0.10 & 2.8 & 10.8 & 0.07 \\
\hline 139.42 & -1.50 & -49.59 & 0.97 & 5.32 & 0.117 & kout & hcs01 & 5.8 & 13.4 & 0.14 & 5.0 & 12.2 & 0.10 & 4.2 & 12.0 & 0.07 \\
\hline 139.53 & -2.81 & -15.26 & 0.73 & 1.29 & 0.088 & kout & hcs01 & 1.5 & 9.7 & 0.12 & 1.2 & 9.0 & 0.08 & 0.9 & 9.2 & 0.04 \\
\hline 139.72 & -0.56 & -39.45 & 0.78 & 3.92 & 0.094 & kout & hcs01 & 4.3 & 12.1 & 0.11 & 3.7 & 11.1 & 0.08 & 3.1 & 11.0 & 0.06 \\
\hline 139.90 & 0.22 & -40.16 & 2.20 & 4.02 & 0.266 & kout & hcs01 & 4.4 & 12.2 & 0.32 & 3.8 & 11.2 & 0.24 & 3.2 & 11.1 & 0.17 \\
\hline 139.91 & 2.61 & -50.80 & 0.77 & 5.58 & 0.093 & kout & hcs01 & 6.1 & 13.7 & 0.11 & 5.2 & 12.5 & 0.08 & 4.5 & 12.3 & 0.06 \\
\hline 140.27 & 2.11 & -12.63 & 3.30 & 1.06 & 0.399 & kout & hcs01 & 1.3 & 9.5 & 0.60 & 1.0 & 8.8 & 0.36 & 0.7 & 9.1 & 0.17 \\
\hline 140.27 & 1.52 & -15.42 & 0.66 & 1.31 & 0.080 & kout & hcs01 & 1.5 & 9.7 & 0.10 & 1.2 & 9.0 & 0.07 & 0.9 & 9.2 & 0.04 \\
\hline 140.27 & 3.48 & -9.92 & 0.73 & 0.82 & 0.088 & kout & hcs01 & 1.0 & 9.3 & 0.13 & 0.8 & 8.6 & 0.08 & 0.5 & 8.9 & 0.03 \\
\hline 140.41 & 4.38 & -13.06 & 0.75 & 1.11 & 0.091 & kout & hcs01 & 1.3 & 9.6 & 0.12 & 1.0 & 8.8 & 0.07 & 0.8 & 9.1 & 0.05 \\
\hline 140.46 & 0.80 & -36.46 & 0.79 & 3.58 & 0.096 & kout & hcs01 & 3.9 & 11.8 & 0.11 & 3.4 & 10.8 & 0.09 & 2.8 & 10.8 & 0.06 \\
\hline 140.60 & -0.10 & -39.20 & 3.22 & 2.50 & .140 & stel & dlp+96 & & & 0.14 & & & 0.14 & & & 0.14 \\
\hline 140.60 & 0.70 & -37.90 & .06 & 2.50 & 0.170 & stel & dlp+ & & & 0.17 & & & 0.17 & & & 0.17 \\
\hline 140.68 & 4.94 & -14.22 & 0.80 & 1.22 & 0.097 & kout & & 1.4 & 9.7 & 0.13 & 1.1 & 8.9 & 0.08 & 0.9 & 9.2 & 0.05 \\
\hline 140.73 & -0.98 & 38.56 & 0.96 & 3.87 & 0.116 & kout & & 4.3 & 12.1 & 0.14 & 3.6 & 11.1 & 0.10 & 3.1 & 11.0 & 0.07 \\
\hline 140.73 & 0.82 & -38.44 & 2.70 & 3.86 & 0.327 & kout & hcs01 & 4.2 & 12.1 & 0.39 & 3.6 & 11.0 & 0.28 & 3.1 & 11.0 & 0.21 \\
\hline 140.77 & -0.16 & -41.09 & 0.71 & 4.21 & 0.086 & kout & hcs01 & 4.6 & 12.4 & 0.10 & 4.0 & 11.3 & 0.08 & 3.3 & 11.3 & 0.05 \\
\hline 140.85 & -0.82 & -40.95 & 1.90 & 4.20 & 0.230 & kout & hcs01 & 4.6 & 12.4 & 0.28 & 3.9 & 11.3 & 0.20 & 3.3 & 11.3 & 0.14 \\
\hline 140.90 & -1.10 & -40.40 & 5.61 & 2.50 & 0.240 & stel & dlp+96 & & & 0.24 & & & 0.24 & & & 0.24 \\
\hline 141.00 & 5.50 & -11.10 & & 0.94 & 0.794 & kout & sod91 & 1.1 & 9.4 & 1.09 & 0.9 & 8.7 & 0.73 & 0.6 & 9.0 & 0.32 \\
\hline 141.46 & -1.18 & -40.79 & 4.60 & 4.23 & 0.557 & kout & hcs01 & 4.6 & 12.5 & 0.66 & 4.0 & 11.4 & 0.50 & 3.3 & 11.3 & 0.34 \\
\hline 141.50 & -1.20 & -40.80 & 3.31 & 2.50 & 0.140 & stel & dlp+96 & & & 0.14 & & & 0.14 & & & 0.14 \\
\hline 142.00 & 1.00 & -15.70 & & 1.37 & 4.467 & kout & sod91 & 1.6 & 9.8 & 6.09 & 1.3 & 9.0 & 4.02 & 1.0 & 9.3 & 2.38 \\
\hline 143.80 & -1.50 & -32.20 & 1.98 & 2.50 & 0.083 & stel & dlp+96 & & & 0.08 & & & 0.08 & & & 0.08 \\
\hline 145.20 & 2.99 & -58.39 & & 8.59 & 0.150 & kout & bw94 & 8.9 & 16.6 & 0.16 & 7.7 & 15.0 & 0.12 & 6.5 & 14.3 & 0.09 \\
\hline 145.30 & -0.25 & -67.60 & 2.40 & 11.00 & 0.120 & kout & dgt94 & 11.8 & 19.4 & 0.14 & 10.2 & 17.4 & 0.10 & 8.3 & 16.1 & 0.07 \\
\hline 145.40 & -0.10 & -70.60 & 1.70 & 12.00 & 0.087 & kout & $\operatorname{dgt} 94$ & 13.0 & 20.5 & 0.10 & 11.2 & 18.4 & 0.08 & 9.1 & 16.8 & 0.05 \\
\hline 146.95 & 0.80 & -67.30 & 2.70 & 12.00 & 0.140 & kout & $\operatorname{dgt} 94$ & 12.7 & 20.3 & 0.16 & 11.0 & 18.2 & 0.12 & 8.9 & 16.7 & 0.08 \\
\hline 148.90 & 0.90 & -59.60 & 3.80 & 10.00 & 0.200 & kout & dgt94 & 10.9 & 18.7 & 0.24 & 9.4 & 16.8 & 0.18 & 7.8 & 15.7 & 0.12 \\
\hline
\end{tabular}


Table A.2. continued.

\begin{tabular}{|c|c|c|c|c|c|c|c|c|c|c|c|c|c|c|c|c|}
\hline $\begin{array}{l}l \\
\left({ }^{\circ}\right) \\
(1)\end{array}$ & $\begin{array}{r}b \\
\left({ }^{\circ}\right) \\
(2)\end{array}$ & $\begin{array}{r}V_{\text {lsr }} \\
\mathrm{km} \mathrm{s}^{-1} \\
\text { (3) }\end{array}$ & $\begin{array}{r}L_{\mathrm{CO}} \\
10^{3} \mathrm{~K} \mathrm{~km} \mathrm{~s}^{-1} \mathrm{pc}^{2} \\
(4)\end{array}$ & $\begin{array}{r}D \\
\mathrm{kpc} \\
(5)\end{array}$ & $\begin{array}{r}M_{\mathrm{GMCs}} \\
10^{5} M_{\odot} \\
(6)\end{array}$ & Mark & Ref. & $\begin{array}{r}D_{8.5} \\
\mathrm{kpc} \\
(9)\end{array}$ & $\begin{array}{l}R_{8.5} \\
\mathrm{kpc} \\
(10)\end{array}$ & $\begin{array}{r}M_{8.5} \\
10^{5} M_{\odot} \\
(11)\end{array}$ & $\begin{array}{l}D_{8.0} \\
\mathrm{kpc} \\
(12)\end{array}$ & $\begin{array}{l}R_{8.0} \\
\mathrm{kpc} \\
(13)\end{array}$ & $\begin{array}{r}M_{8.0} \\
10^{5} M_{\odot} \\
(14)\end{array}$ & $\begin{array}{l}D_{8.4} \\
\mathrm{kpc} \\
(15)\end{array}$ & $\begin{array}{l}R_{8.4} \\
\mathrm{kpc} \\
(16)\end{array}$ & $\begin{array}{r}M_{8.4} \\
10^{5} M_{\odot} \\
(17)\end{array}$ \\
\hline 150.15 & 1.10 & -55.90 & 2.10 & 10.00 & 0.110 & kout & dgt94 & 10.3 & 18.2 & 0.12 & 8.9 & 16.3 & 0.09 & 7.4 & 15.3 & 0.06 \\
\hline 151.23 & 1.02 & -53.86 & & 9.91 & 0.140 & kout & bw94 & 10.2 & 18.1 & 0.15 & 8.8 & 16.3 & 0.11 & 7.3 & 15.3 & 0.08 \\
\hline 151.50 & -1.00 & -30.60 & & 3.82 & 10.471 & kout & sod91 & 4.2 & 12.4 & 12.66 & 3.6 & 11.3 & 9.30 & 3.0 & 11.2 & 6.46 \\
\hline 154.00 & -2.00 & -14.40 & & 1.63 & 2.188 & kout & sod91 & 1.9 & 10.2 & 2.97 & 1.5 & 9.4 & 1.85 & 1.2 & 9.6 & 1.19 \\
\hline 154.35 & 2.61 & -35.80 & 7.40 & 6.00 & 0.895 & stel & $\operatorname{css} 90$ & & & 0.89 & & & 0.89 & & & 0.89 \\
\hline 154.64 & 2.45 & -38.70 & 3.00 & 6.00 & 0.363 & stel & $\operatorname{css} 90$ & & & 0.36 & & & 0.36 & & & 0.36 \\
\hline 156.89 & 0.21 & -47.44 & & 11.33 & 0.140 & kout & bw94 & 11.7 & 19.8 & 0.15 & 10.1 & 17.7 & 0.11 & 8.2 & 16.3 & 0.07 \\
\hline 173.71 & 2.70 & -17.40 & 7.60 & 1.80 & 0.920 & stel & $\operatorname{css} 90$ & 1.8 & 10.3 & 0.92 & 1.8 & 9.8 & 0.92 & & & \\
\hline 189.03 & 0.78 & 2.90 & 2.20 & 1.50 & 0.266 & stel & $\operatorname{css} 90$ & 1.5 & 10.0 & 0.27 & 1.5 & 9.5 & 0.27 & & & \\
\hline 190.05 & 0.53 & 8.40 & 0.88 & 1.50 & 0.106 & stel & $\operatorname{css} 90$ & 1.5 & 10.0 & 0.11 & 1.5 & 9.5 & 0.11 & & & \\
\hline 194.00 & -0.50 & 20.90 & & 5.90 & 2.800 & kout & mab97 & 6.1 & 14.5 & 2.99 & 5.3 & 13.2 & 2.26 & 4.5 & 13.0 & 1.63 \\
\hline 194.25 & -0.50 & 20.00 & & 5.40 & 1.520 & kout & mab97 & 5.5 & 13.9 & 1.58 & 4.8 & 12.7 & 1.20 & 4.1 & 12.5 & 0.88 \\
\hline 194.50 & -1.26 & 14.40 & & 3.20 & 0.210 & kout & mab97 & 3.4 & 11.8 & 0.24 & 2.9 & 10.8 & 0.17 & 2.5 & 11.0 & 0.13 \\
\hline 194.50 & -1.00 & 22.60 & & 6.40 & 0.560 & kout & mab97 & 6.6 & 15.0 & 0.60 & 5.7 & 13.6 & 0.44 & 4.9 & 13.3 & 0.33 \\
\hline 195.50 & 0.00 & 20.40 & & 4.90 & 0.190 & kout & mab97 & 5.1 & 13.4 & 0.21 & 4.3 & 12.2 & 0.15 & 3.7 & 12.1 & 0.11 \\
\hline 195.75 & -0.13 & 32.60 & & 11.00 & 1.920 & kout & mab97 & 11.3 & 19.6 & 2.03 & 9.8 & 17.6 & 1.52 & 8.1 & 16.4 & 1.04 \\
\hline 196.00 & 0.50 & 20.20 & & 4.60 & 0.480 & kout & mab97 & 4.8 & 13.2 & 0.52 & 4.1 & 12.0 & 0.38 & 3.5 & 11.9 & 0.28 \\
\hline 196.45 & -1.68 & 17.70 & 3.00 & 3.80 & 0.363 & stel & $\operatorname{css} 90$ & & & 0.36 & & & 0.36 & & & 0.36 \\
\hline 196.83 & -3.10 & 25.30 & 1.10 & 4.80 & 0.133 & stel & $\operatorname{css} 90$ & & & 0.13 & & & 0.13 & & & 0.13 \\
\hline 197.00 & 0.75 & 19.60 & & 4.00 & 0.890 & kout & mab97 & 4.2 & 12.6 & 0.98 & 3.6 & 11.5 & 0.72 & 3.1 & 11.5 & 0.53 \\
\hline 203.00 & 1.00 & 32.40 & & 5.70 & 0.200 & kout & mab97 & 5.9 & 14.1 & 0.21 & 5.1 & 12.8 & 0.16 & 4.3 & 12.6 & 0.11 \\
\hline 204.50 & -0.50 & 26.80 & & 4.00 & 0.190 & kout & mab97 & 4.1 & 12.4 & 0.20 & 3.5 & 11.3 & 0.15 & 3.0 & 11.3 & 0.11 \\
\hline 204.63 & 0.13 & 45.70 & & 9.30 & 0.300 & kout & mab97 & 9.6 & 17.7 & 0.32 & 8.3 & 15.9 & 0.24 & 7.0 & 15.1 & 0.17 \\
\hline 205.00 & 0.75 & 33.30 & & 5.30 & 0.620 & kout & mab97 & 5.5 & 13.7 & 0.67 & 4.7 & 12.5 & 0.49 & 4.0 & 12.3 & 0.35 \\
\hline 205.25 & 0.00 & 20.70 & & 2.70 & 0.110 & kout & mab97 & 2.9 & 11.2 & 0.13 & 2.4 & 10.3 & 0.09 & 2.1 & 10.4 & 0.07 \\
\hline 206.00 & -0.50 & 20.70 & & 2.60 & 0.090 & kout & mab97 & 2.8 & 11.1 & 0.10 & 2.4 & 10.2 & 0.08 & 2.0 & 10.3 & 0.05 \\
\hline 207.50 & 0.25 & 34.40 & & 4.90 & 0.230 & kout & mab97 & 5.1 & 13.3 & 0.25 & 4.4 & 12.1 & 0.19 & 3.8 & 12.0 & 0.14 \\
\hline 207.75 & 1.00 & 39.50 & & 6.00 & 0.150 & kout & mab97 & 6.3 & 14.3 & 0.17 & 5.4 & 13.0 & 0.12 & 4.6 & 12.7 & 0.09 \\
\hline 208.50 & 0.75 & 39.20 & & 5.70 & 0.350 & kout & mab97 & 6.0 & 14.0 & 0.39 & 5.1 & 12.8 & 0.28 & 4.4 & 12.5 & 0.21 \\
\hline 208.75 & -1.75 & 51.10 & & 8.90 & 0.590 & kout & mab97 & 9.2 & 17.2 & 0.63 & 8.0 & 15.5 & 0.48 & 6.8 & 14.8 & 0.34 \\
\hline 209.50 & -1.25 & 24.90 & & 2.90 & 0.140 & kout & mab97 & 3.1 & 11.3 & 0.16 & 2.6 & 10.4 & 0.11 & 2.3 & 10.5 & 0.09 \\
\hline 209.50 & -1.00 & 44.90 & & 6.90 & 0.260 & kout & mab97 & 7.1 & 15.1 & 0.28 & 6.1 & 13.7 & 0.20 & 5.2 & 13.3 & 0.15 \\
\hline 209.50 & 0.50 & 29.80 & & 3.70 & 0.370 & kout & mab97 & 3.9 & 12.1 & 0.41 & 3.3 & 11.0 & 0.29 & 2.8 & 11.1 & 0.21 \\
\hline 210.00 & -2.25 & 22.20 & & 2.50 & 0.130 & kout & mab97 & 2.7 & 10.9 & 0.15 & 2.3 & 10.0 & 0.11 & 1.9 & 10.2 & 0.08 \\
\hline 210.00 & -0.75 & 35.00 & & 4.60 & 0.280 & kout & mab97 & 4.8 & 12.9 & 0.30 & 4.1 & 11.7 & 0.22 & 3.5 & 11.7 & 0.16 \\
\hline 210.13 & -1.75 & 39.80 & & 5.50 & 0.300 & kout & mab97 & 5.7 & 13.7 & 0.32 & 4.9 & 12.5 & 0.24 & 4.2 & 12.4 & 0.17 \\
\hline 210.13 & -1.50 & 37.80 & & 5.10 & 0.180 & kout & mab97 & 5.3 & 13.4 & 0.19 & 4.6 & 12.2 & 0.15 & 3.9 & 12.1 & 0.11 \\
\hline 210.38 & 0.00 & 35.90 & & 4.70 & 1.010 & kout & mab97 & 4.9 & 12.9 & 1.10 & 4.2 & 11.8 & 0.81 & 3.6 & 11.7 & 0.59 \\
\hline 211.04 & 1.18 & 54.29 & & 8.88 & 0.140 & kout & bw94 & 9.1 & 17.0 & 0.15 & 7.9 & 15.3 & 0.11 & 6.6 & 14.6 & 0.08 \\
\hline 211.25 & -1.75 & 42.20 & & 5.80 & 0.350 & kout & mab97 & 6.0 & 14.0 & 0.37 & 5.2 & 12.7 & 0.28 & 4.4 & 12.5 & 0.20 \\
\hline 211.25 & -1.00 & 43.80 & & 6.10 & 0.180 & kout & mab97 & 6.3 & 14.3 & 0.19 & 5.5 & 13.0 & 0.15 & 4.7 & 12.7 & 0.11 \\
\hline 211.25 & -0.50 & 21.70 & & 2.40 & 0.090 & kout & mab97 & 2.5 & 10.7 & 0.10 & 2.1 & 9.9 & 0.07 & 1.8 & 10.1 & 0.05 \\
\hline 211.50 & 1.00 & 45.40 & & 6.40 & 1.190 & kout & mab97 & 6.6 & 14.6 & 1.27 & 5.7 & 13.2 & 0.94 & 4.9 & 12.9 & 0.70 \\
\hline 212.25 & -1.00 & 27.50 & & 3.10 & 0.150 & kout & mab97 & 3.3 & 11.4 & 0.17 & 2.8 & 10.4 & 0.12 & 2.4 & 10.6 & 0.09 \\
\hline 212.25 & -1.00 & 44.30 & & 6.00 & 1.110 & kout & mab97 & 6.2 & 14.1 & 1.19 & 5.3 & 12.8 & 0.87 & 4.6 & 12.6 & 0.65 \\
\hline 213.00 & 1.25 & 42.90 & & 5.50 & 1.040 & kout & mab97 & 5.8 & 13.7 & 1.16 & 5.0 & 12.5 & 0.86 & 4.2 & 12.3 & 0.61 \\
\hline 213.25 & -2.00 & 37.10 & & 4.50 & 0.210 & kout & mab97 & 4.7 & 12.7 & 0.23 & 4.0 & 11.6 & 0.17 & 3.5 & 11.6 & 0.13 \\
\hline 214.00 & 0.75 & 44.10 & & 5.60 & 0.940 & kout & mab97 & 5.8 & 13.7 & 1.01 & 5.0 & 12.5 & 0.75 & 4.3 & 12.3 & 0.55 \\
\hline 214.50 & -1.75 & 27.60 & & 2.90 & 0.390 & kout & mab97 & 3.1 & 11.2 & 0.45 & 2.6 & 10.3 & 0.31 & 2.2 & 10.4 & 0.22 \\
\hline 214.50 & 1.00 & 46.00 & & 5.90 & 1.130 & kout & mab97 & 6.1 & 13.9 & 1.21 & 5.2 & 12.7 & 0.88 & 4.5 & 12.4 & 0.66 \\
\hline 215.00 & 1.00 & 47.60 & & 6.10 & 2.480 & kout & mab97 & 6.3 & 14.1 & 2.65 & 5.4 & 12.8 & 1.94 & 4.6 & 12.6 & 1.41 \\
\hline 215.25 & -0.25 & 29.20 & & 3.10 & 0.400 & kout & mab97 & 3.3 & 11.3 & 0.45 & 2.8 & 10.4 & 0.33 & 2.4 & 10.5 & 0.24 \\
\hline 215.50 & -2.25 & 43.40 & & 5.20 & 0.100 & kout & mab97 & 5.4 & 13.3 & 0.11 & 4.7 & 12.1 & 0.08 & 4.1 & 12.0 & 0.06 \\
\hline 215.50 & 0.25 & 48.70 & & 6.20 & 0.520 & kout & mab97 & 6.4 & 14.2 & 0.55 & 5.5 & 12.9 & 0.41 & 4.7 & 12.7 & 0.30 \\
\hline 215.75 & 1.25 & 50.70 & & 6.50 & 0.720 & kout & mab97 & 6.8 & 14.5 & 0.79 & 5.9 & 13.2 & 0.59 & 5.0 & 12.9 & 0.43 \\
\hline 216.00 & 0.50 & 45.70 & & 5.50 & 0.180 & kout & mab97 & 5.8 & 13.6 & 0.20 & 5.0 & 12.4 & 0.15 & 4.2 & 12.2 & 0.10 \\
\hline 216.25 & 0.00 & 28.20 & & 2.90 & 0.100 & kout & mab97 & 3.1 & 11.1 & 0.11 & 2.6 & 10.2 & 0.08 & 2.2 & 10.3 & 0.06 \\
\hline 216.50 & 0.50 & 46.70 & & 5.70 & 0.130 & kout & mab97 & 5.9 & 13.7 & 0.14 & 5.1 & 12.4 & 0.10 & 4.3 & 12.3 & 0.07 \\
\hline 216.50 & 1.25 & 48.00 & & 5.90 & 0.580 & kout & mab97 & 6.1 & 13.9 & 0.62 & 5.3 & 12.6 & 0.47 & 4.5 & 12.4 & 0.34 \\
\hline 216.50 & -2.50 & 22.80 & & 2.14 & 12.303 & kout & sod91 & 2.4 & 10.5 & 15.47 & 2.0 & 9.7 & 10.75 & 1.7 & 9.9 & 7.76 \\
\hline 216.75 & -1.00 & 31.30 & & 3.30 & 0.140 & kout & mab97 & 3.4 & 11.4 & 0.15 & 2.9 & 10.5 & 0.11 & 2.5 & 10.6 & 0.08 \\
\hline 217.00 & 0.50 & 50.90 & & 6.30 & 1.340 & kout & mab97 & 6.6 & 14.3 & 1.47 & 5.7 & 13.0 & 1.10 & 4.8 & 12.7 & 0.78 \\
\hline 217.13 & -0.13 & 53.80 & & 6.90 & 0.220 & kout & mab97 & 7.1 & 14.8 & 0.23 & 6.2 & 13.4 & 0.18 & 5.3 & 13.1 & 0.13 \\
\hline 217.25 & 0.00 & 26.70 & & 2.70 & 0.700 & kout & mab97 & 2.8 & 10.9 & 0.75 & 2.4 & 10.0 & 0.55 & 2.0 & 10.2 & 0.38 \\
\hline
\end{tabular}


Table A.2. continued.

\begin{tabular}{|c|c|c|c|c|c|c|c|c|c|c|c|c|c|c|c|c|}
\hline $\begin{array}{r}l \\
\left({ }^{\circ}\right) \\
(1)\end{array}$ & $\begin{array}{r}b \\
\left({ }^{\circ}\right) \\
(2)\end{array}$ & $\begin{array}{r}V_{\mathrm{lsr}} \\
\mathrm{km} \mathrm{s}^{-1} \\
(3)\end{array}$ & $\begin{array}{r}L_{\mathrm{CO}} \\
10^{3} \mathrm{~K} \mathrm{~km} \mathrm{~s}^{-1} \mathrm{pc}^{2} \\
(4)\end{array}$ & $\begin{array}{r}D \\
\mathrm{kpc} \\
(5)\end{array}$ & $\begin{array}{r}M_{\mathrm{GMCs}} \\
10^{5} M_{\odot} \\
(6)\end{array}$ & Mark & Ref. & $\begin{array}{r}D_{8.5} \\
\mathrm{kpc} \\
(9)\end{array}$ & $\begin{array}{l}R_{8.5} \\
\mathrm{kpc} \\
(10)\end{array}$ & $\begin{array}{r}M_{8.5} \\
10^{5} M_{\odot} \\
(11)\end{array}$ & $\begin{array}{l}D_{8.0} \\
\mathrm{kpc} \\
(12)\end{array}$ & $\begin{array}{l}R_{8.0} \\
\mathrm{kpc} \\
(13)\end{array}$ & $\begin{array}{r}M_{8.0} \\
10^{5} M_{\odot} \\
(14)\end{array}$ & $\begin{array}{l}D_{8.4} \\
\mathrm{kpc} \\
(15)\end{array}$ & $\begin{array}{l}R_{8.4} \\
\mathrm{kpc} \\
(16)\end{array}$ & $\begin{array}{r}M_{8.4} \\
10^{5} M_{\odot} \\
(17)\end{array}$ \\
\hline 217.25 & 0.00 & 27.70 & & 2.80 & 0.360 & kout & mab97 & 3.0 & 11.0 & 0.41 & 2.5 & 10.1 & 0.29 & 2.1 & 10.3 & 0.20 \\
\hline 217.38 & -1.38 & 50.50 & & 6.20 & 0.280 & kout & mab97 & 6.4 & 14.2 & 0.30 & 5.5 & 12.9 & 0.22 & 4.8 & 12.6 & 0.17 \\
\hline 217.38 & 0.25 & 50.50 & & 6.20 & 1.550 & kout & mab97 & 6.4 & 14.1 & 1.65 & 5.5 & 12.9 & 1.22 & 4.8 & 12.6 & 0.93 \\
\hline 218.00 & -0.25 & 27.70 & & 2.70 & 0.630 & kout & mab97 & 2.9 & 11.0 & 0.73 & 2.5 & 10.1 & 0.54 & 2.1 & 10.2 & 0.38 \\
\hline 218.00 & 0.25 & 22.80 & & 2.20 & 0.200 & kout & mab97 & 2.4 & 10.5 & 0.24 & 2.0 & 9.6 & 0.17 & 1.6 & 9.8 & 0.11 \\
\hline 218.25 & -2.00 & 26.30 & & 2.60 & 0.360 & kout & mab97 & 2.7 & 10.8 & 0.39 & 2.3 & 9.9 & 0.28 & 2.0 & 10.1 & 0.21 \\
\hline 218.25 & -2.00 & 32.30 & & 3.30 & 0.720 & kout & mab97 & 3.5 & 11.4 & 0.81 & 3.0 & 10.5 & 0.60 & 2.5 & 10.6 & 0.41 \\
\hline 218.75 & -0.50 & 56.10 & & 7.00 & 0.170 & kout & mab97 & 7.3 & 14.9 & 0.18 & 6.3 & 13.5 & 0.14 & 5.4 & 13.1 & 0.10 \\
\hline 218.75 & -0.50 & 59.60 & & 7.80 & 0.220 & kout & mab97 & 8.0 & 15.6 & 0.23 & 6.9 & 14.1 & 0.17 & 5.9 & 13.6 & 0.13 \\
\hline 219.00 & -1.88 & 31.30 & & 3.10 & 0.290 & kout & mab97 & 3.3 & 11.3 & 0.33 & 2.8 & 10.3 & 0.24 & 2.4 & 10.5 & 0.17 \\
\hline 219.75 & -2.00 & 30.90 & & 3.00 & 0.210 & kout & mab97 & 3.2 & 11.2 & 0.24 & 2.7 & 10.2 & 0.17 & 2.3 & 10.4 & 0.12 \\
\hline 220.25 & -2.00 & 33.80 & & 3.40 & 0.310 & kout & mab97 & 3.6 & 11.5 & 0.35 & 3.0 & 10.5 & 0.24 & 2.6 & 10.6 & 0.18 \\
\hline 221.75 & 0.25 & 63.80 & & 8.00 & 0.200 & kout & mab97 & 8.2 & 15.6 & 0.21 & 7.2 & 14.2 & 0.16 & 6.1 & 13.7 & 0.12 \\
\hline 222.50 & -0.25 & 51.30 & & 5.60 & 0.160 & kout & mab97 & 5.9 & 13.4 & 0.18 & 5.1 & 12.2 & 0.13 & 4.3 & 12.1 & 0.09 \\
\hline 222.75 & -2.00 & 39.70 & & 4.00 & 0.290 & kout & mab97 & 4.2 & 11.9 & 0.32 & 3.6 & 10.9 & 0.23 & 3.1 & 11.0 & 0.17 \\
\hline 223.75 & -2.00 & 63.20 & & 7.50 & 0.190 & kout & mab97 & 7.8 & 15.1 & 0.21 & 6.7 & 13.7 & 0.15 & 5.8 & 13.3 & 0.11 \\
\hline 224.00 & -2.00 & 17.60 & & 1.47 & 4.677 & kout & sod91 & 1.7 & 9.8 & 6.26 & 1.4 & 9.0 & 4.24 & 1.1 & 9.3 & 2.62 \\
\hline 228.00 & -1.00 & 36.50 & & 3.40 & 0.110 & kout & mab97 & 3.6 & 11.2 & 0.12 & 3.1 & 10.3 & 0.09 & 2.6 & 10.4 & 0.06 \\
\hline 229.75 & -2.50 & 41.30 & & 3.90 & 0.120 & kout & mab97 & 4.1 & 11.6 & 0.13 & 3.5 & 10.6 & 0.10 & 3.0 & 10.7 & 0.07 \\
\hline 230.00 & -1.00 & 39.20 & & 3.70 & 0.230 & kout & mab97 & 3.9 & 11.4 & 0.26 & 3.3 & 10.4 & 0.18 & 2.8 & 10.5 & 0.13 \\
\hline 230.25 & -2.75 & 42.00 & & 4.00 & 0.380 & kout & mab97 & 4.2 & 11.6 & 0.42 & 3.6 & 10.6 & 0.31 & 3.1 & 10.7 & 0.23 \\
\hline 231.13 & 0.00 & 43.40 & & 4.10 & 0.210 & kout & mab97 & 4.3 & 11.7 & 0.23 & 3.7 & 10.7 & 0.17 & 3.2 & 10.8 & 0.13 \\
\hline 231.50 & -1.00 & 43.10 & & 4.10 & 0.910 & kout & mab97 & 4.3 & 11.6 & 1.00 & 3.7 & 10.7 & 0.74 & 3.2 & 10.7 & 0.55 \\
\hline 232.00 & 0.13 & 57.20 & & 5.80 & 0.360 & kout & mab97 & 6.0 & 13.1 & 0.39 & 5.2 & 11.9 & 0.29 & 4.5 & 11.8 & 0.22 \\
\hline 232.00 & -2.50 & 42.30 & & 3.81 & 14.454 & kout & sod91 & 4.2 & 11.6 & 17.56 & 3.6 & 10.6 & 12.90 & 3.1 & 10.7 & 9.57 \\
\hline 232.25 & -1.00 & 44.60 & & 4.20 & 0.370 & kout & mab97 & 4.4 & 11.7 & 0.41 & 3.8 & 10.8 & 0.30 & 3.3 & 10.8 & 0.23 \\
\hline 232.75 & -3.25 & 40.60 & & 3.80 & 0.790 & kout & mab97 & 4.0 & 11.4 & 0.88 & 3.4 & 10.4 & 0.63 & 2.9 & 10.5 & 0.46 \\
\hline 233.00 & -1.00 & 47.00 & & 4.50 & 0.410 & kout & mab97 & 4.7 & 11.9 & 0.45 & 4.0 & 10.9 & 0.32 & 3.5 & 11.0 & 0.25 \\
\hline 233.00 & -0.75 & 45.60 & & 4.30 & 1.430 & kout & mab97 & 4.5 & 11.8 & 1.57 & 3.9 & 10.8 & 1.18 & 3.4 & 10.9 & 0.89 \\
\hline 233.00 & 0.00 & 18.90 & & 1.57 & 1.549 & kout & sod91 & 1.8 & 9.7 & 2.04 & 1.5 & 9.0 & 1.41 & 1.1 & 9.2 & 0.76 \\
\hline 233.25 & -1.75 & 51.60 & & 5.00 & 0.160 & kout & mab97 & 5.3 & 12.4 & 0.18 & 4.5 & 11.3 & 0.13 & 3.9 & 11.3 & 0.10 \\
\hline 234.00 & -0.25 & 43.70 & & 4.10 & 1.930 & kout & mab97 & 4.3 & 11.6 & 2.12 & 3.7 & 10.6 & 1.57 & 3.2 & 10.7 & 1.18 \\
\hline 234.25 & 0.88 & 76.30 & & 8.60 & 0.930 & kout & mab97 & 8.8 & 15.4 & 0.97 & 7.7 & 14.0 & 0.75 & 6.7 & 13.5 & 0.56 \\
\hline 234.50 & -0.25 & 43.40 & & 4.10 & 1.540 & kout & mab97 & 4.3 & 11.5 & 1.69 & 3.7 & 10.6 & 1.25 & 3.2 & 10.6 & 0.94 \\
\hline 235.00 & 0.50 & 47.90 & & 4.60 & 0.160 & kout & mab97 & 4.8 & 11.9 & 0.17 & 4.1 & 10.9 & 0.13 & 3.6 & 10.9 & 0.10 \\
\hline 235.38 & -1.75 & 80.80 & & 9.30 & 0.950 & kout & mab97 & 9.5 & 16.0 & 0.99 & 8.3 & 14.4 & 0.76 & 7.2 & 13.9 & 0.57 \\
\hline 235.50 & 0.75 & 48.30 & & 4.60 & 0.160 & kout & mab97 & 4.8 & 11.9 & 0.17 & 4.2 & 10.9 & 0.13 & 3.6 & 10.9 & 0.10 \\
\hline 235.75 & -1.50 & 52.60 & & 5.10 & 0.190 & kout & mab97 & 5.3 & 12.3 & 0.21 & 4.6 & 11.3 & 0.15 & 4.0 & 11.3 & 0.12 \\
\hline 236.38 & -1.75 & 77.60 & & 8.60 & 1.230 & kout & mab97 & 8.9 & 15.3 & 1.32 & 7.7 & 13.9 & 0.99 & 6.7 & 13.4 & 0.75 \\
\hline 236.38 & -1.50 & 29.20 & & 2.60 & 0.200 & kout & mab97 & 2.8 & 10.3 & 0.23 & 2.4 & 9.5 & 0.17 & 2.0 & 9.7 & 0.12 \\
\hline 236.75 & -0.50 & 56.60 & & 5.60 & 0.130 & kout & mab97 & 5.8 & 12.7 & 0.14 & 5.0 & 11.5 & 0.10 & 4.4 & 11.5 & 0.08 \\
\hline 237.00 & -2.00 & 23.20 & & 2.10 & 0.190 & kout & mab97 & 2.3 & 9.9 & 0.23 & 1.9 & 9.1 & 0.16 & 1.5 & 9.4 & 0.10 \\
\hline 237.13 & -2.38 & 57.10 & & 5.60 & 0.260 & kout & mab97 & 5.9 & 12.7 & 0.29 & 5.1 & 11.6 & 0.22 & 4.5 & 11.5 & 0.17 \\
\hline 237.25 & -1.25 & 74.40 & & 8.00 & 1.050 & kout & mab97 & 8.3 & 14.7 & 1.13 & 7.2 & 13.4 & 0.85 & 6.3 & 13.0 & 0.65 \\
\hline 237.50 & -0.25 & 55.90 & & 5.50 & 0.180 & kout & mab97 & 5.7 & 12.5 & 0.19 & 4.9 & 11.4 & 0.14 & 4.3 & 11.4 & 0.11 \\
\hline 237.75 & -1.00 & 22.40 & & 2.00 & 0.260 & kout & mab97 & 2.2 & 9.9 & 0.31 & 1.8 & 9.1 & 0.21 & 1.4 & 9.3 & 0.13 \\
\hline 238.38 & -3.25 & 65.20 & & 6.70 & 0.490 & kout & mab97 & 6.9 & 13.5 & 0.52 & 6.0 & 12.3 & 0.39 & 5.3 & 12.1 & 0.31 \\
\hline 239.13 & -3.50 & 23.00 & & 2.10 & 0.190 & kout & mab97 & 2.3 & 9.9 & 0.23 & 1.9 & 9.1 & 0.16 & 1.5 & 9.4 & 0.10 \\
\hline 239.13 & -1.88 & 83.00 & & 9.40 & 2.460 & kout & mab97 & 9.6 & 15.8 & 2.57 & 8.4 & 14.3 & 1.96 & 7.3 & 13.8 & 1.48 \\
\hline 239.50 & -1.88 & 79.90 & & 8.80 & 0.410 & kout & mab97 & 9.1 & 15.3 & 0.44 & 7.9 & 13.8 & 0.33 & 6.9 & 13.4 & 0.25 \\
\hline 240.25 & -1.75 & 23.90 & & 2.20 & 0.180 & kout & mab97 & 2.4 & 9.9 & 0.21 & 2.0 & 9.1 & 0.15 & 1.6 & 9.4 & 0.10 \\
\hline 240.25 & 0.00 & 68.10 & & 7.00 & 0.340 & kout & mab97 & 7.3 & 13.7 & 0.37 & 6.3 & 12.4 & 0.28 & 5.5 & 12.2 & 0.21 \\
\hline 240.25 & 0.50 & 68.70 & & 7.10 & 0.230 & kout & mab97 & 7.4 & 13.7 & 0.25 & 6.4 & 12.5 & 0.19 & 5.6 & 12.3 & 0.14 \\
\hline 241.00 & -1.25 & 66.10 & & 6.80 & 2.760 & kout & mab97 & 7.0 & 13.4 & 2.92 & 6.1 & 12.2 & 2.22 & 5.3 & 12.0 & 1.68 \\
\hline 241.75 & -0.63 & 68.40 & & 7.10 & 3.350 & kout & mab97 & 7.3 & 13.6 & 3.54 & 6.4 & 12.4 & 2.72 & 5.6 & 12.2 & 2.08 \\
\hline 242.00 & -0.88 & 66.00 & & 6.70 & 1.720 & kout & mab97 & 7.0 & 13.3 & 1.88 & 6.1 & 12.1 & 1.43 & 5.3 & 12.0 & 1.08 \\
\hline 242.25 & -2.75 & 21.50 & & 2.00 & 0.190 & kout & mab97 & 2.2 & 9.7 & 0.23 & 1.8 & 9.0 & 0.15 & 1.4 & 9.3 & 0.09 \\
\hline 242.25 & -2.75 & 26.20 & & 2.40 & 0.320 & kout & mab97 & 2.7 & 10.0 & 0.41 & 2.2 & 9.2 & 0.27 & 1.8 & 9.5 & 0.18 \\
\hline 242.25 & 0.13 & 69.80 & & 7.20 & 1.720 & kout & mab97 & 7.5 & 13.7 & 1.87 & 6.5 & 12.5 & 1.40 & 5.7 & 12.3 & 1.08 \\
\hline 242.63 & 0.13 & 71.90 & & 7.50 & 1.210 & kout & mab97 & 7.8 & 13.9 & 1.31 & 6.8 & 12.7 & 0.99 & 5.9 & 12.4 & 0.75 \\
\hline 242.88 & -0.50 & 64.30 & & 6.50 & 1.580 & kout & mab97 & 6.8 & 13.1 & 1.73 & 5.9 & 11.9 & 1.30 & 5.2 & 11.8 & 1.01 \\
\hline 242.88 & 0.00 & 55.80 & & 5.50 & 0.500 & kout & mab97 & 5.7 & 12.2 & 0.54 & 5.0 & 11.2 & 0.41 & 4.4 & 11.2 & 0.32 \\
\hline 243.00 & -3.00 & 22.20 & & 2.10 & 0.170 & kout & mab97 & 2.3 & 9.8 & 0.20 & 1.9 & 9.0 & 0.14 & 1.5 & 9.3 & 0.09 \\
\hline 243.25 & -0.75 & 66.90 & & 6.90 & 0.760 & kout & mab97 & 7.1 & 13.3 & 0.80 & 6.2 & 12.1 & 0.61 & 5.4 & 12.0 & 0.47 \\
\hline 244.25 & -2.50 & 70.40 & & 7.30 & 0.130 & kout & mab97 & 7.6 & 13.6 & 0.14 & 6.6 & 12.4 & 0.11 & 5.8 & 12.2 & 0.08 \\
\hline
\end{tabular}


Table A.2. continued.

\begin{tabular}{|c|c|c|c|c|c|c|c|c|c|c|c|c|c|c|c|c|c|}
\hline $\begin{array}{l}l \\
\left({ }^{\circ}\right) \\
(1)\end{array}$ & $\begin{array}{r}b \\
\left({ }^{\circ}\right) \\
(2)\end{array}$ & $\begin{array}{r}V_{\mathrm{lsr}} \\
\mathrm{km} \mathrm{s}^{-1} \\
(3)\end{array}$ & $10^{3} \mathrm{~K} \mathrm{~km} \mathrm{~s}^{-}$ & $\begin{array}{r}L_{\mathrm{CO}} \\
\mathrm{pc}^{2} \\
(4)\end{array}$ & $\begin{array}{r}D \\
\mathrm{kpc} \\
(5)\end{array}$ & $\begin{array}{r}M_{\mathrm{GMCs}} \\
10^{5} M_{\odot} \\
(6)\end{array}$ & Mark & Ref. & $\begin{array}{r}D_{8.5} \\
\mathrm{kpc} \\
(9)\end{array}$ & $\begin{array}{l}R_{8.5} \\
\mathrm{kpc} \\
(10)\end{array}$ & $\begin{array}{r}M_{8.5} \\
10^{5} M_{\odot} \\
(11)\end{array}$ & $\begin{array}{l}D_{8.0} \\
\mathrm{kpc} \\
(12)\end{array}$ & $\begin{array}{l}R_{8.0} \\
\mathrm{kpc} \\
(13)\end{array}$ & $\begin{array}{r}M_{8.0} \\
10^{5} M_{\odot} \\
(14)\end{array}$ & $\begin{array}{l}D_{8.4} \\
\mathrm{kpc} \\
(15)\end{array}$ & $\begin{array}{l}R_{8.4} \\
\mathrm{kpc} \\
(16)\end{array}$ & $\begin{array}{r}M_{8.4} \\
10^{5} M_{\odot} \\
(17)\end{array}$ \\
\hline 244.50 & -2.00 & 92.60 & & & 10.80 & 0.750 & kout & mab97 & 11.1 & 16.6 & 0.79 & 9.7 & 15.0 & 0.61 & 8.4 & 14.3 & 0.45 \\
\hline 244.75 & -0.25 & 62.50 & & & 6.30 & 0.190 & kout & mab97 & 6.6 & 12.8 & 0.21 & 5.7 & 11.7 & 0.16 & 5.0 & 11.6 & 0.12 \\
\hline 246.75 & -0.50 & 21.40 & & & 2.10 & 0.620 & kout & mab97 & 2.4 & 9.7 & 0.81 & 1.9 & 8.9 & 0.51 & 1.5 & 9.2 & 0.32 \\
\hline 247.75 & -0.75 & 29.90 & & & 3.00 & 0.090 & kout & mab97 & 3.2 & 10.2 & 0.10 & 2.7 & 9.4 & 0.07 & 2.3 & 9.6 & 0.05 \\
\hline 248.00 & 0.50 & 27.00 & & & 2.70 & 0.230 & kout & mab97 & 3.0 & 10.0 & 0.28 & 2.5 & 9.2 & 0.20 & 2.0 & 9.4 & 0.13 \\
\hline 249.50 & -3.88 & 89.90 & & & 10.20 & 0.480 & kout & mab97 & 10.6 & 15.7 & 0.52 & 9.3 & 14.2 & 0.40 & 8.2 & 13.7 & 0.31 \\
\hline 249.88 & -3.13 & 98.00 & & & 11.60 & 1.500 & kout & mab97 & 12.0 & 16.9 & 1.61 & 10.5 & 15.2 & 1.23 & 9.2 & 14.5 & 0.94 \\
\hline 250.38 & -1.00 & 66.40 & & & 7.00 & 0.200 & kout & mab97 & 7.3 & 12.9 & 0.22 & 6.4 & 11.8 & 0.17 & 5.7 & 11.7 & 0.13 \\
\hline 250.63 & -3.63 & 89.60 & & & 10.20 & 0.320 & kout & mab97 & 10.5 & 15.6 & 0.34 & 9.2 & 14.1 & 0.26 & 8.2 & 13.6 & 0.21 \\
\hline 251.25 & 2.00 & 54.80 & & & 5.70 & 0.260 & kout & mab97 & 6.0 & 11.9 & 0.29 & 5.2 & 10.9 & 0.22 & 4.6 & 10.9 & 0.17 \\
\hline 52.00 & 3.50 & 8.50 & & & 1.00 & 0.093 & kout & sod91 & 1.4 & 9.0 & 0.18 & 0.9 & 8.3 & 0.08 & 0.5 & 8.7 & 0.02 \\
\hline 252.00 & -3.50 & 15.00 & & & 1.68 & 0.891 & kout & sod91 & 2.0 & 9.3 & 1.26 & 1.6 & 8.6 & 0.81 & 1.1 & 8.9 & 0.38 \\
\hline 252.08 & -4.24 & 95.14 & & & 11.22 & 0.100 & kout & bw94 & 11.5 & 16.3 & 0.11 & 10.1 & 14.7 & 0.08 & 8.9 & 14.1 & 0.06 \\
\hline 253.00 & -2.93 & 99.60 & & 0.91 & 11.50 & 0.111 & kout & nomf05 & 12.3 & 16.8 & 0.13 & 10.8 & 15.2 & 0.10 & 9.5 & 14.5 & 0.08 \\
\hline 253.00 & -1.50 & 5.90 & & & 0.74 & 0.263 & kout & sod91 & 1.1 & 8.9 & 0.58 & 0.7 & 8.2 & 0.24 & 0.2 & 8.6 & 0.02 \\
\hline 253.23 & -2.73 & 98.90 & & 0.99 & 11.30 & 0.121 & kout & nomf05 & 12.1 & 16.7 & 0.14 & 10.7 & 15.1 & 0.11 & 9.4 & 14.4 & 0.08 \\
\hline 253.53 & -3.17 & 107.20 & & 0.94 & 12.80 & 0.114 & kout & nomf05 & 13.7 & 18.1 & 0.13 & 12.1 & 16.3 & 0.10 & 10.6 & 15.3 & 0.08 \\
\hline 253.63 & 0.00 & 34.70 & & & 3.80 & 2.640 & kout & mab97 & 4.1 & 10.4 & 3.07 & 3.5 & 9.6 & 2.24 & 3.0 & 9.8 & 1.65 \\
\hline 253.75 & 0.00 & 34.60 & & & 3.80 & 1.690 & kout & mab97 & 4.1 & 10.4 & 1.97 & 3.5 & 9.6 & 1.43 & 3.0 & 9.8 & 1.05 \\
\hline 254.25 & -2.25 & 36.10 & & & 4.00 & 0.350 & kout & mab97 & 4.3 & 10.5 & 0.40 & 3.6 & 9.6 & 0.28 & 3.2 & 9.8 & 0.22 \\
\hline 254.25 & -1.00 & 76.60 & & & 8.50 & 0.330 & kout & mab97 & 8.8 & 13.8 & 0.35 & 7.7 & 12.5 & 0.27 & 6.9 & 12.3 & 0.22 \\
\hline 5.25 & -1.00 & 80.10 & & & 9.00 & 1.180 & kout & mab97 & 9.3 & 14.1 & 1.26 & 8.2 & 12.8 & 0.98 & 7.3 & 12.6 & 0.78 \\
\hline 255.38 & -0.75 & 86.00 & & & 9.90 & 1.440 & kout & mab97 & 10.1 & 14.8 & 1.50 & 8.9 & 13.4 & 1.16 & 8.0 & 13.0 & 0.94 \\
\hline 255.47 & -3.93 & 108.70 & & 2.85 & 13.20 & 0.348 & kout & nomf05 & 14.1 & 18.2 & 0.40 & 12.4 & 16.4 & 0.31 & 10.9 & 15.4 & 0.24 \\
\hline 5.75 & 1.00 & 35.70 & & & 4.10 & 0.150 & kout & mab97 & 4.3 & 10.4 & 0.16 & 3.7 & 9.6 & 0.12 & 3.2 & 9.8 & 0.09 \\
\hline 56.00 & 2.50 & 9.80 & & & 1.31 & 1.738 & kout & sod91 & 1.7 & 9.1 & 2.93 & 1.2 & 8.4 & 1.46 & 0.7 & 8.7 & 0.50 \\
\hline 256.12 & -1.50 & 87.50 & & & 10.10 & 0.950 & kout & mab97 & 10.4 & 14.9 & 1.01 & 9.2 & 13.5 & 0.79 & 8.2 & 13.2 & 0.63 \\
\hline 256.72 & -2.00 & 44.40 & & & 5.00 & 0.280 & kout & mab97 & 5.3 & 11.0 & 0.31 & 4.6 & 10.1 & 0.24 & 4.1 & 10.2 & 0.19 \\
\hline 257.00 & -0.50 & 53.00 & & & 6.00 & 0.810 & kout & mab97 & 6.2 & 11.6 & 0.86 & 5.4 & 10.6 & 0.66 & 4.8 & 10.7 & 0.52 \\
\hline 257.37 & -1.10 & 90.90 & & 1.55 & 10.30 & 0.190 & kout & nomf05 & 11.0 & 15.3 & 0.22 & 9.7 & 13.9 & 0.17 & 8.7 & 13.4 & 0.14 \\
\hline 257.50 & 2.38 & 93.10 & & & 11.10 & 1.100 & kout & mab97 & 11.4 & 15.6 & 1.16 & 10.0 & 14.1 & 0.89 & 9.0 & 13.6 & 0.72 \\
\hline 7.50 & 2.00 & 41.50 & & & 4.80 & 0.380 & kout & mab97 & 5.1 & 10.8 & 0.43 & 4.4 & 9.9 & 0.32 & 3.9 & 10.1 & 0.25 \\
\hline 57.50 & -0.25 & 55.50 & & & 6.30 & 0.190 & kout & mab97 & 6.5 & 11.8 & 0.20 & 5.7 & 10.8 & 0.16 & 5.1 & 10.8 & 0.12 \\
\hline 7.53 & -2.33 & 94.80 & & 6.84 & 10.90 & 0.836 & kout & nomf05 & 11.6 & 15.8 & 0.95 & 10.3 & 14.3 & 0.75 & 9.2 & 13.8 & 0.60 \\
\hline 7.57 & -2.07 & 1.30 & & 5.02 & 0.40 & 0.613 & kout & nomf05 & 11.1 & 15.4 & 0.70 & 9.8 & 13.9 & 0.54 & 8.8 & 13.4 & 0.44 \\
\hline .83 & 2.63 & 1.10 & & 4.57 & 10.40 & 0.558 & kout & nomf05 & 11.1 & 15.3 & 0.64 & 9.8 & 13.9 & 0.50 & 8.8 & 13.4 & 0.40 \\
\hline .40 & 2.93 & 100.90 & & 1.91 & 11.90 & 0.233 & kout & nomf05 & 12.7 & 16.7 & 0.27 & 11.2 & 15.0 & 0.21 & 10.0 & 14.4 & 0.16 \\
\hline .50 & 1.50 & 7.20 & & & 7.70 & 0.530 & kout & ab97 & 7.9 & 12.7 & 0.56 & 7.0 & 11.6 & 0.44 & 6.3 & 11.6 & 0.35 \\
\hline .50 & 1.00 & 1.20 & & & 9.40 & 0.960 & kout & ab97 & 9.7 & 14.1 & 1.02 & 8.6 & 12.8 & 0.80 & 7.7 & 12.6 & 0.64 \\
\hline 8.60 & 3.87 & 105.10 & & 0.87 & 12.70 & 0.107 & kout & nomf05 & 13.5 & 17.3 & 0.12 & 11.9 & 15.6 & 0.09 & 10.6 & 14.8 & 0.07 \\
\hline 258.93 & 1.03 & 90.60 & & 0.81 & 10.40 & 0.099 & kout & nomf05 & 11.1 & 15.2 & 0.11 & 9.8 & 13.8 & 0.09 & 8.8 & 13.3 & 0.07 \\
\hline 259.00 & -1.75 & 58.50 & & & 6.70 & 1.290 & kout & mab97 & 7.0 & 12.0 & 1.41 & 6.1 & 11.0 & 1.07 & 5.5 & 11.0 & 0.87 \\
\hline 259.25 & -2.50 & 54.50 & & & 6.30 & 1.160 & kout & mab97 & 6.6 & 11.7 & 1.27 & 5.8 & 10.7 & 0.98 & 5.2 & 10.8 & 0.79 \\
\hline 259.37 & -1.90 & 95.80 & & 1.28 & 11.20 & 0.156 & kout & nomf05 & 11.9 & 15.9 & 0.18 & 10.5 & 14.4 & 0.14 & 9.4 & 13.8 & 0.11 \\
\hline 259.75 & -1.25 & 62.90 & & & 7.30 & 0.840 & kout & mab97 & 7.6 & 12.3 & 0.91 & 6.6 & 11.3 & 0.69 & 6.0 & 11.2 & 0.57 \\
\hline 259.83 & -1.57 & 94.20 & & 1.00 & 11.00 & 0.122 & kout & nomf05 & 11.7 & 15.6 & 0.14 & 10.3 & 14.2 & 0.11 & 9.3 & 13.6 & 0.09 \\
\hline 260.00 & 0.00 & 60.70 & & & 7.10 & 0.680 & kout & mab97 & 7.3 & 12.2 & 0.72 & 6.4 & 11.1 & 0.55 & 5.8 & 11.1 & 0.45 \\
\hline 260.07 & -1.53 & 91.70 & & 2.05 & 10.70 & 0.251 & kout & nomf05 & 11.3 & 15.3 & 0.28 & 10.0 & 13.9 & 0.22 & 9.0 & 13.4 & 0.18 \\
\hline 260.50 & -0.75 & 57.20 & & & 6.70 & 0.860 & kout & mab97 & 7.0 & 11.9 & 0.94 & 6.1 & 10.9 & 0.71 & 5.6 & 10.9 & 0.60 \\
\hline 260.63 & 0.38 & 55.30 & & & 6.50 & 0.950 & kout & & 6.8 & 11.7 & .04 & 6.0 & 10.7 & 0.81 & 5.4 & 10.8 & 0.66 \\
\hline 260.75 & -2.50 & 64.10 & & & 7.50 & 0.390 & kout & & 7.8 & 12.4 & 0.42 & 6.9 & 11.4 & 0.33 & 6.2 & 11.3 & 0.27 \\
\hline 261.00 & 0.75 & 70.00 & & & 8.30 & & kout & & 8.5 & 12.9 & 58 & 7.5 & 11.8 & 1.23 & 6.8 & 11.7 & 1.01 \\
\hline 261.00 & 2.50 & 13.70 & & & 2.10 & 2.951 & kout & & 2.5 & 9.2 & 4.18 & 2.0 & 8.5 & 2.68 & 1.4 & 8.8 & 1.31 \\
\hline 261.27 & 5.03 & 102.20 & & 2.03 & 12.40 & 0.248 & kout & nomf05 & 13.2 & 16.8 & 0.28 & 11.7 & 15.1 & 0.22 & 10.4 & 14.4 & 0.17 \\
\hline 261.40 & 2.00 & 102.90 & & 1.94 & 12.50 & 0.237 & kout & nomf05 & 13.3 & 16.8 & 0.27 & 11.8 & 15.2 & 0.21 & 10.5 & 14.5 & 0.17 \\
\hline 61.50 & 1.25 & 55.40 & & & 6.60 & 0.220 & kout & mab97 & 6.9 & 11.7 & 0.24 & 6.1 & 10.7 & 0.19 & 5.5 & 10.8 & 0.15 \\
\hline 261.80 & 3.03 & 103.00 & & 1.20 & 12.50 & 0.147 & kout & nomf05 & 13.4 & 16.8 & 0.17 & 11.8 & 15.2 & 0.13 & 10.6 & 14.5 & 0.11 \\
\hline 261.83 & 1.47 & 101.40 & & 1.12 & 12.30 & 0.137 & kout & nomf05 & 13.1 & 16.6 & 0.16 & 11.6 & 15.0 & 0.12 & 10.3 & 14.3 & 0.10 \\
\hline 262.17 & -2.20 & 98.60 & & 0.83 & 11.90 & 0.102 & kout & nomf05 & 12.6 & 16.2 & 0.11 & 11.2 & 14.6 & 0.09 & 10.0 & 14.0 & 0.07 \\
\hline 262.25 & -2.00 & 59.70 & & & 7.20 & 0.780 & kout & mab97 & 7.5 & 12.0 & 0.85 & 6.6 & 11.0 & 0.66 & 6.0 & 11.0 & 0.54 \\
\hline 262.75 & -0.50 & 59.90 & & & 7.30 & 0.490 & kout & mab97 & 7.5 & 12.1 & 0.52 & 6.6 & 11.0 & 0.40 & 6.0 & 11.0 & 0.33 \\
\hline 263.00 & -2.50 & 62.10 & & & 7.50 & 0.230 & kout & mab97 & 7.8 & 12.2 & 0.25 & 6.9 & 11.2 & 0.19 & 6.3 & 11.2 & 0.16 \\
\hline 263.60 & -4.00 & 111.00 & & 2.51 & 14.10 & 0.307 & kout & nomf05 & 15.1 & 18.1 & 0.35 & 13.3 & 16.3 & 0.27 & 11.8 & 15.3 & 0.22 \\
\hline 264.00 & -1.50 & 29.40 & & & 4.20 & 0.800 & kout & mab97 & 4.5 & 10.0 & 0.92 & 3.9 & 9.2 & 0.69 & 3.4 & 9.5 & 0.52 \\
\hline
\end{tabular}


Table A.2. continued.

\begin{tabular}{|c|c|c|c|c|c|c|c|c|c|c|c|c|c|c|c|c|}
\hline $\begin{array}{r}l \\
\left({ }^{\circ}\right) \\
(1) \\
\end{array}$ & $\begin{array}{r}b \\
\left({ }^{\circ}\right) \\
(2) \\
\end{array}$ & $\begin{array}{r}V_{\mathrm{lsr}} \\
\mathrm{km} \mathrm{s}^{-1} \\
(3) \\
\end{array}$ & $\begin{array}{r}L_{\mathrm{CO}} \\
10^{3} \mathrm{~K} \mathrm{~km} \mathrm{~s}^{-1} \mathrm{pc}^{2} \\
(4) \\
\end{array}$ & $\begin{array}{r}D \\
\mathrm{kpc} \\
(5) \\
\end{array}$ & $\begin{array}{r}M_{\mathrm{GMCs}} \\
10^{5} M_{\odot} \\
(6) \\
\end{array}$ & Mark & Ref. & $\begin{array}{l}D_{8.5} \\
\mathrm{kpc} \\
(9) \\
\end{array}$ & $\begin{array}{l}R_{8.5} \\
\mathrm{kpc} \\
(10) \\
\end{array}$ & $\begin{array}{r}M_{8.5} \\
10^{5} M_{\odot} \\
(11) \\
\end{array}$ & $\begin{array}{l}D_{8.0} \\
\mathrm{kpc} \\
(12) \\
\end{array}$ & $\begin{array}{l}R_{8.0} \\
\mathrm{kpc} \\
(13) \\
\end{array}$ & $\begin{array}{r}M_{8.0} \\
10^{5} M_{\odot} \\
(14) \\
\end{array}$ & $\begin{array}{l}D_{8.4} \\
\mathrm{kpc} \\
(15) \\
\end{array}$ & $\begin{array}{l}R_{8.4} \\
\mathrm{kpc} \\
(16) \\
\end{array}$ & $\begin{array}{r}M_{8.4} \\
10^{5} M_{\odot} \\
(17) \\
\end{array}$ \\
\hline 265.00 & -1.50 & 29.40 & & 4.30 & 0.480 & kout & mab97 & 4.6 & 10.0 & 0.55 & 4.0 & 9.2 & 0.42 & 3.5 & 9.5 & 0.32 \\
\hline 265.00 & 0.00 & 47.10 & & 6.20 & 0.150 & kout & mab97 & 6.4 & 11.1 & 0.16 & 5.6 & 10.2 & 0.12 & 5.1 & 10.3 & 0.10 \\
\hline 265.25 & -1.25 & 65.90 & & 8.30 & 0.360 & kout & mab97 & 8.5 & 12.5 & 0.38 & 7.5 & 11.4 & 0.29 & 6.9 & 11.4 & 0.25 \\
\hline 265.50 & -1.50 & 27.00 & & 4.10 & 0.150 & kout & mab97 & 4.4 & 9.9 & 0.17 & 3.8 & 9.1 & 0.13 & 3.3 & 9.4 & 0.10 \\
\hline 265.53 & -2.00 & 90.80 & 4.20 & 11.10 & 0.513 & kout & nomf05 & 11.8 & 15.1 & 0.58 & 10.5 & 13.6 & 0.46 & 9.5 & 13.2 & 0.38 \\
\hline 266.50 & 3.00 & 65.50 & & 8.30 & 0.340 & kout & mab97 & 8.6 & 12.5 & 0.37 & 7.7 & 11.4 & 0.29 & 7.0 & 11.4 & 0.24 \\
\hline 266.50 & -2.00 & 24.20 & & 4.00 & 0.110 & kout & mab97 & 4.3 & 9.7 & 0.13 & 3.6 & 9.0 & 0.09 & 3.2 & 9.2 & 0.07 \\
\hline 266.75 & -2.00 & 57.60 & & 7.50 & 0.280 & kout & mab97 & 7.8 & 11.8 & 0.30 & 6.9 & 10.8 & 0.24 & 6.3 & 10.9 & 0.20 \\
\hline 266.75 & -0.75 & 31.20 & & 4.70 & 0.180 & kout & mab97 & 5.0 & 10.1 & 0.20 & 4.4 & 9.3 & 0.16 & 3.9 & 9.5 & 0.12 \\
\hline 267.00 & -0.75 & 19.20 & & 3.50 & 0.320 & kout & mab97 & 3.8 & 9.5 & 0.38 & 3.2 & 8.8 & 0.27 & 2.7 & 9.0 & 0.19 \\
\hline 267.25 & -2.00 & 18.10 & & 3.40 & 0.160 & kout & mab97 & 3.7 & 9.4 & 0.19 & 3.1 & 8.7 & 0.13 & 2.6 & 9.0 & 0.09 \\
\hline 268.50 & -2.50 & 62.60 & & 8.30 & 1.120 & kout & mab97 & 8.6 & 12.2 & 1.20 & 7.6 & 11.2 & 0.94 & 7.0 & 11.2 & 0.80 \\
\hline 269.00 & -1.25 & 62.10 & & 8.30 & 0.720 & kout & mab97 & 8.6 & 12.2 & 0.77 & 7.6 & 11.1 & 0.60 & 7.0 & 11.1 & 0.51 \\
\hline 269.00 & -0.50 & 42.50 & & 6.20 & 1.070 & kout & mab97 & 6.5 & 10.8 & 1.18 & 5.7 & 9.9 & 0.90 & 5.2 & 10.1 & 0.75 \\
\hline 269.00 & -0.50 & 46.60 & & 6.70 & 0.480 & kout & mab97 & 6.9 & 11.1 & 0.51 & 6.1 & 10.1 & 0.40 & 5.6 & 10.3 & 0.34 \\
\hline 269.25 & -1.75 & 79.10 & & 10.40 & 0.910 & kout & mab97 & 10.7 & 13.7 & 0.96 & 9.5 & 12.5 & 0.76 & 8.7 & 12.3 & 0.64 \\
\hline 269.88 & -0.13 & 74.90 & & 0.00 & 1.870 & kout & mab97 & 10.2 & 13.3 & 1.95 & 9.1 & 12.1 & 1.55 & 8.4 & 11.9 & 1.32 \\
\hline 270.90 & -0.50 & 52.00 & & .80 & 2.200 & kout & gcbt88 & 7.8 & 11.4 & 2.89 & 6.9 & 10.5 & 2.27 & 6.4 & 10.5 & 1.95 \\
\hline 279.90 & -1.60 & 35.00 & & 7.10 & 1.000 & kout & 88 & 7.6 & 10.4 & 1.15 & 6.8 & 9.5 & 0.92 & 6.4 & 9.7 & 0.81 \\
\hline 281.40 & -1.10 & -5.00 & & 3.20 & 22.500 & stel & gcbt88 & & & 22.50 & & & 22.50 & & & 22.50 \\
\hline 282.00 & -0.80 & 17.00 & & 6.10 & 2.700 & kout & gcbt 88 & 6.2 & 9.4 & 2.79 & 5.4 & 8.7 & 2.12 & 5.1 & 8.9 & 1.89 \\
\hline 282.90 & 1.30 & -19.00 & & 2.20 & 0.700 & ktan & gcbt88 & 1.9 & 8.3 & 0.52 & 1.8 & 7.8 & 0.47 & 1.9 & 8.2 & 0.52 \\
\hline 282.90 & -0.70 & -5.00 & & 3.20 & 2.800 & kfar & gcbt88 & 3.4 & 8.4 & 3.16 & 2.3 & 7.8 & 1.45 & 1.9 & 8.3 & 0.99 \\
\hline 283.80 & 0.00 & -5.00 & & 4.00 & 6.300 & stel & gcbt88 & & & 6.30 & & & 6.30 & & & 6.30 \\
\hline 284.50 & -0.20 & 12.00 & & 6.60 & 20.300 & kout & gcbt 88 & 6.2 & 9.2 & 17.91 & 5.4 & 8.5 & 13.59 & 5.1 & 8.8 & 12.12 \\
\hline 285.30 & 0.00 & 0.00 & & 5.30 & 4.500 & kfar & gcbt88 & 4.9 & 8.6 & 3.85 & 4.2 & 8.0 & 2.83 & 3.7 & 8.3 & 2.19 \\
\hline 286.00 & 0.00 & -20.20 & & 34 & 19.498 & kout & sod91 & & & & & & & 2.3 & 8.2 & 18.84 \\
\hline 286.40 & -0.30 & 14.00 & & 50 & 8.300 & kout & gcbt88 & 6.8 & 9.3 & 6.82 & 6.1 & 8.6 & 5.49 & 5.8 & 8.8 & 4.96 \\
\hline 287.50 & -0.50 & -19.00 & & 2.70 & 700 & stel & gcbt88 & & & 6.70 & & & 6.70 & & & 6.70 \\
\hline 287.53 & 0.97 & -4.20 & 3.34 & 2.60 & 117 & stel & $\mathrm{dkm}+08$ & & & 0.12 & & & 0.12 & & & 0.12 \\
\hline 287.60 & 8.00 & -27.80 & 3.29 & 2.60 & 0.115 & stel & $\mathrm{dkm}+08$ & & & 0.12 & & & 0.12 & & & 0.12 \\
\hline 3.60 & 1.50 & -22.00 & & 3.20 & 500 & ktan & gcbt 88 & 2.7 & 8.1 & 2.49 & 2.6 & 7.6 & 2.31 & 2.7 & 8.0 & 2.49 \\
\hline 8.80 & 1.07 & -16.90 & 56.05 & 2.60 & 1.960 & stel & $\mathrm{dkm}+08$ & & & 1.96 & & & 1.96 & & & 1.96 \\
\hline 289.00 & -0.50 & 20.20 & & 7.67 & 85.114 & kout & sod91 & 8.0 & 9.6 & 92.60 & 7.2 & 8.9 & 75.00 & 7.0 & 9.1 & 70.89 \\
\hline 9.30 & -0.60 & 22.00 & & 7.90 & 31.200 & stel & gcbt88 & & & 31.20 & & & 31.20 & & & 31.20 \\
\hline 290.20 & -0.20 & -1.00 & & 6.80 & 700 & kfar & gcbt88 & 6.1 & 8.6 & 2.17 & 5.4 & 8.0 & 1.70 & 5.1 & 8.3 & 1.52 \\
\hline 290.60 & -0.20 & 15.00 & & 8.70 & 4.300 & kout & gcbt88 & 7.9 & 9.3 & 3.55 & 7.1 & 8.6 & 2.86 & 6.9 & 8.9 & 2.70 \\
\hline 291.50 & -1.50 & -25.40 & & 3.12 & 36.308 & kout & sod91 & & & & & & & 3.1 & 7.9 & 35.84 \\
\hline 291.60 & -0.40 & 15.00 & & 7.20 & 4.100 & stel & gcbt 88 & & & 4.10 & & & 4.10 & & & 4.10 \\
\hline 291.60 & -0.50 & 29.00 & & 0.30 & 12.500 & kout & gcbt88 & 9.4 & 10.1 & 10.41 & 8.6 & 9.3 & 8.71 & 8.4 & 9.5 & 8.31 \\
\hline 292.60 & -0.30 & 4.00 & & 8.10 & 12.000 & kfar & gcbt88 & 7.3 & 8.8 & 9.75 & 6.5 & 8.2 & 7.73 & 6.4 & 8.4 & 7.49 \\
\hline 293.30 & -1.40 & -25.00 & & 2.40 & 7.100 & stel & gcbt88 & & & 7.10 & & & 7.10 & & & 7.10 \\
\hline 294.00 & 0.90 & 32.00 & & 11.30 & 12.200 & kout & gcbt88 & 10.3 & 10.3 & 10.14 & 9.3 & 9.5 & 8.26 & 9.2 & 9.7 & 8.09 \\
\hline 294.40 & 0.70 & -3.00 & & 8.00 & 12.200 & kfar & 88 & 7.0 & 8.5 & 9.34 & 6.3 & 7.9 & 7.57 & 6.2 & 8.2 & 7.33 \\
\hline 295.10 & .80 & 26.00 & & .00 & & kout & & 10.0 & 10.0 & 3.72 & 9.1 & 9.2 & 3.08 & 9.0 & 9.4 & 3.01 \\
\hline 297.40 & .50 & 2.00 & & 20 & 32.100 & kout & & 10.1 & 9.8 & 26.10 & 9.2 & 9.0 & 21.66 & 9.2 & 9.2 & 21.66 \\
\hline 7.50 & -0.50 & 25.40 & & .14 & & kout & & 10.5 & 10.0 & 234.59 & 9.5 & 9.2 & 2.03 & 9.5 & 9.4 & 192.03 \\
\hline .60 & 0.10 & -35.00 & & 4.70 & & ktan & & 4.1 & 7.5 & 20.17 & 3.8 & 7.0 & 17.32 & 4.0 & 7.4 & 19.19 \\
\hline 80 & -0.20 & 25.00 & & & & kout & & 10.7 & 10.0 & 24.49 & 9.8 & 9.2 & 20.54 & 9.8 & 9.4 & 20.54 \\
\hline 9.40 & 0.10 & -6.00 & & & 4.600 & kfar & gcbt88 & 8.0 & 8.4 & 3.33 & 7.3 & 7.8 & 2.77 & 7.3 & 8.1 & 2.77 \\
\hline 299.50 & -0.50 & -39.70 & & 4.19 & 57.544 & kout & sod91 & & & & & & & 4.1 & 7.4 & 55.10 \\
\hline 300.30 & -0.20 & 31.00 & & 2.90 & 000 & kout & gcbt 88 & 11.6 & 10.4 & 5.66 & 10.6 & 9.6 & 4.73 & 10.6 & 9.7 & 4.73 \\
\hline 300.60 & -0.20 & 10.00 & & 1.10 & 4.200 & kout & gcbt88 & 9.8 & 9.1 & 3.27 & 9.0 & 8.4 & 2.76 & 9.0 & 8.7 & 2.76 \\
\hline 301.80 & 0.00 & 24.00 & & 2.70 & 4.200 & kout & gcbt88 & 11.3 & 10.0 & 3.33 & 10.4 & 9.2 & 2.82 & 10.4 & 9.3 & 2.82 \\
\hline 302.30 & -0.70 & 32.00 & & 3.60 & 29.400 & kout & gcbt88 & 12.2 & 10.5 & 23.66 & 11.2 & 9.7 & 19.94 & 11.2 & 9.8 & 19.94 \\
\hline 303.90 & -0.40 & 29.00 & & 3.70 & 46.200 & kout & gcbt88 & 12.3 & 10.3 & 37.24 & 11.3 & 9.5 & 31.43 & 11.3 & 9.6 & 31.43 \\
\hline 306.90 & -0.50 & 25.00 & & 4.20 & 5.600 & kout & gcbt 88 & 12.6 & 10.1 & 4.41 & 11.6 & 9.3 & 3.74 & 11.7 & 9.5 & 3.80 \\
\hline 308.00 & -0.70 & 32.00 & & 15.10 & 21.000 & kout & gcbt88 & 13.5 & 10.7 & 16.79 & 12.4 & 9.8 & 14.16 & 12.5 & 9.9 & 14.39 \\
\hline
\end{tabular}


Table A.2. continued.

\begin{tabular}{|c|c|c|c|c|c|c|c|c|c|c|c|c|c|c|c|c|}
\hline $\begin{array}{l}l \\
\left({ }^{\circ}\right) \\
(1)\end{array}$ & $\begin{array}{r}b \\
\left({ }^{\circ}\right) \\
(2)\end{array}$ & $\begin{array}{r}V_{\text {lsr }} \\
\mathrm{km} \mathrm{s}^{-1} \\
\text { (3) }\end{array}$ & $\begin{array}{r}L_{\mathrm{CO}} \\
10^{3} \mathrm{~K} \mathrm{~km} \mathrm{~s}^{-1} \mathrm{pc}^{2} \\
(4)\end{array}$ & $\begin{array}{r}D \\
\mathrm{kpc} \\
(5)\end{array}$ & $\begin{array}{r}M_{\mathrm{GMCS}} \\
10^{5} M_{\odot} \\
(6)\end{array}$ & Mark & Ref. & $\begin{array}{r}D_{8.5} \\
\mathrm{kpc} \\
(9)\end{array}$ & $\begin{array}{l}R_{8.5} \\
\mathrm{kpc} \\
(10)\end{array}$ & $\begin{array}{r}M_{8.5} \\
10^{5} M_{\odot} \\
(11)\end{array}$ & $\begin{array}{l}D_{8.0} \\
\mathrm{kpc} \\
(12)\end{array}$ & $\begin{array}{l}R_{8.0} \\
\mathrm{kpc} \\
(13)\end{array}$ & $\begin{array}{r}M_{8.0} \\
10^{5} M_{\odot} \\
(14)\end{array}$ & $\begin{array}{l}D_{8.4} \\
\mathrm{kpc} \\
(15)\end{array}$ & $\begin{array}{l}R_{8.4} \\
\mathrm{kpc} \\
(16)\end{array}$ & $\begin{array}{r}M_{8.4} \\
10^{5} M_{\odot} \\
(17)\end{array}$ \\
\hline 311.30 & -0.30 & 27.00 & & 15.50 & 109.200 & kout & gcbt88 & 13.8 & 10.4 & 86.56 & 12.7 & 9.6 & 73.31 & 12.8 & 9.6 & 74.47 \\
\hline 313.20 & -0.30 & 42.00 & & 17.60 & 18.200 & kout & gcbt88 & 15.9 & 11.8 & 14.85 & 14.6 & 10.8 & 12.52 & 14.6 & 10.7 & 12.52 \\
\hline 314.00 & -0.10 & 28.00 & & 16.40 & 4.200 & kout & bt 88 & 14.5 & 10.6 & 3.28 & 13.4 & 9.7 & 2.80 & 13.5 & 9.8 & 2.85 \\
\hline & -0.30 & 14.00 & & 15.40 & 11.200 & kout & bt 88 & 13.5 & 9.5 & 8.61 & 12.4 & 8.8 & 7.26 & 12.7 & 8.9 & 7.62 \\
\hline & 0.30 & 30.00 & & 17.60 & 2.800 & kout & bt 88 & 15.7 & 10.9 & 2.23 & 14.4 & 10.0 & 1.87 & 14.6 & 10.0 & 1.93 \\
\hline .50 & .50 & 26.00 & & 17.80 & 12.600 & kout & bt 88 & 15.8 & 10.7 & 9.93 & 14.6 & 9.8 & 8.48 & 14.8 & 9.8 & 8.71 \\
\hline 5.30 & 0.10 & 28.00 & & 19.30 & 19.600 & kout & cbt88 & 17.1 & 11.2 & 15.39 & 15.8 & 10.3 & 13.14 & 16.0 & 10.2 & 13.47 \\
\hline 328.50 & -0.10 & 30.00 & & 20.40 & 5.600 & kout & t88 & 18.2 & 11.8 & 4.46 & 16.8 & 10.8 & 3.80 & 17.0 & 10.7 & 3.89 \\
\hline 335.50 & -0.50 & 27.00 & & 22.00 & 0.600 & kout & gcbt 88 & 19.7 & 12.4 & 0.48 & 18.1 & 11.4 & 0.41 & 18.3 & 11.1 & 0.42 \\
\hline
\end{tabular}

References: bw94: Brand \& Wouterloot (1994); css90: Carpenter et al. (1990); dect86: Dame et al. (1986); dbt90: Digel et al. (1990); dgt94: Digel et al. (1994); dlp+96: Digel et al. (1996); dkm+08: Dawson et al. (2008); gcbt88: Grabelsky et al. (1988); hcs01: Heyer et al. (2001); mk88: Mead \& Kutner (1988); mab97: May et al. (1997); nomf05: Nakagawa et al. (2005); sod91: Sodroski (1991); srby87: Solomon et al. (1987); tllw07: Tian et al. (2007). 